\title{
Grainyhead-like-2 inhibits the co-activator p300, suppressing tubulogenesis and the epithelial-mesenchymal transition
}

Phillip M. Pifer

Follow this and additional works at: https://researchrepository.wvu.edu/etd

\section{Recommended Citation}

Pifer, Phillip M., "Grainyhead-like-2 inhibits the co-activator p300, suppressing tubulogenesis and the epithelial-mesenchymal transition" (2016). Graduate Theses, Dissertations, and Problem Reports. 6420. https://researchrepository.wvu.edu/etd/6420

This Dissertation is protected by copyright and/or related rights. It has been brought to you by the The Research Repository @ WVU with permission from the rights-holder(s). You are free to use this Dissertation in any way that is permitted by the copyright and related rights legislation that applies to your use. For other uses you must obtain permission from the rights-holder(s) directly, unless additional rights are indicated by a Creative Commons license in the record and/ or on the work itself. This Dissertation has been accepted for inclusion in WVU Graduate Theses, Dissertations, and Problem Reports collection by an authorized administrator of The Research Repository @ WVU. For more information, please contact researchrepository@mail.wvu.edu. 
Grainyhead-like-2 inhibits the co-activator p300, suppressing tubulogenesis and the epithelial-mesenchymal transition

Phillip M. Pifer

Dissertation submitted to the School of Medicine at West Virginia University in partial fulfillment of the requirements for the degree of Doctor of Philosophy in Cancer Cell Biology

\author{
Karen Martin, PhD, Committee Chairperson \\ Michael Schaller, PhD \\ Peter Mathers, PhD \\ Peter Stoilov, PhD \\ David M. Smith, PhD \\ Steven M. Frisch, PhD, Mentor
}

Cancer Cell Biology Program

Morgantown, West Virginia

2016

Keywords: EMT, grainyhead-like-2, p300, CBP, histone acetyltransferase, MDCK, tubulogenesis 


\title{
Abstract \\ Grainyhead-like-2 inhibits the co-activator p300, suppressing tubulogenesis and the epithelial-mesenchymal transition
}

\author{
Phillip M. Pifer
}

Tumor progression and developmental morphogenesis require transient or stable Epithelial-Mesenchymal transitions (EMT) to modulate epithelial junctional complexes allowing programmed migration, invasion, and anoikis resistance of cells. Grainyhead-like-2 (GRHL2), an epithelial master-regulatory transcription factor, suppresses EMT and causes a mesenchymalepithelial transition to the default epithelial phenotype. This body of work investigates the role of GRHL2 in kidney cancer, in kidney development using a kidney specific GRHL2 knockout mouse model, and in tubulogenesis using Madin-Darby Canine Kidney (MDCK) cells. In kidney cancer, GRHL2 was not expressed in the normal kidney proximal tubule epithelial cells which are thought to be the progenitors of clear cell renal cell carcinomas. However, GRHL2 could function as biomarker to differentiate renal cell carcinoma subtypes. In kidney development, a large amount of evidence suggests Grhl2 is a critical regulator of this process. However, kidney specific Grhl2 knockout mice did not elucidate the role of Grhl2 in the developing kidney for technical reasons, and this area needs to be further investigated. In the MDCK model, GRHL2 was required for cystogenesis, but it suppressed Hepatocyte Growth Factor-induced tubulogenesis, a process requiring transient, partial EMT. Subsequent results demonstrated that GRHL2 suppressed tubulogenesis by inhibiting the histone acetyltransferase co-activator, p300, thereby preventing the induction of matrix metalloproteases and other p300-dependent genes required for tubulogenesis. A thirteen amino acid region (425-437aa) of GRHL2 was required and sufficient for the inhibition of p300 histone acetyltransferase activity, the suppression of tubulogenesis and the interference with EMT. These results demonstrate that p300 is required for EMT plasticity occurring in tubulogenesis or tumor progression, and that GRHL2 suppresses EMT in both contexts, through inhibition of p300. 


\section{Dedication}

This dissertation is dedicated to all my family members who have fought cancer, especially my grandfather Harry Higgs. I would also like to dedicate this work to my family and friends whose unwavering support allows me to pursue my passions. I would specifically like to thank my parents, Michael and Ann Pifer, my brother and his family, Joseph, Miranda, and Aven, and my amazing fiancé, Colleen Beatty. I would also like to thank my grandmother, Judy Higgs, for her careful reading of the dissertation. 


\section{Acknowledgments}

Steven Frisch for his unparalleled mentorship. His research career has been incredibly impactful, and his dedication to scientific advancement is unmatched. He demonstrates critical thinking skills, passion, and persistence that scientific research requires, and I have learned a wealth of knowledge from him.

Fred Minnear and the $\mathrm{MD} / \mathrm{PhD}$ admissions committee for my acceptance into the program.

My dissertation committee including Karen Martin, Peter Mathers, Peter Stoilov, Mike Schaller, and David Smith for their unwavering support and valuable scientific insight throughout my pursuit of this degree.

Special thanks to Mike Lutz laying the foundation for my love of chemistry during my high school education.

Thanks to Jeffrey Petersen, who convinced me to become a Chemistry major during my first day of college, and whose mentorship encouraged me to pursue a career in both medicine and research.

Thanks to Justin Legleiter, who was an exceptional undergraduate research mentor and was the first person to encourage me into a $\mathrm{MD} / \mathrm{PhD}$ dual doctoral degree.

I would like to thank my labmate Joshua Farris, for the constant companionship during the ups and downs of the last four years pursuing a Ph.D. degree. I would also like to thank previous lab members, Benjamin Cieply and Sun Hee Park, for their excellent training and advice.

Finally, I would like to thank the $\mathbf{M D} / \mathbf{P h D}$ program for both financial support to attain these degrees as well as consistent mentorship from both faculty and students to guide me through the difficult stages of my training. 


\section{Table of Contents}

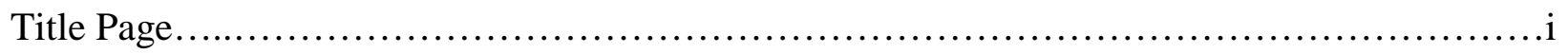

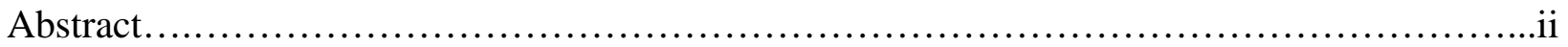

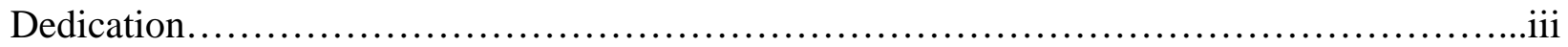

Acknowledgements..................................................................

Table of Contents......................................................................

List of Abbreviations.............................................................

Chapter 1 - Introduction and Literature Review..........................................

Chapter 2 - Grainyhead-like-2 Inhibits the Co-activator p300, Suppressing Tubulogenesis and the Epithelial-Mesenchymal Transition..............................................60

Chapter 3 - GRHL2 in Kidney Cancer and Development...............................138

Chapter 4 - Overall Discussion and Future Directions................................171

Chapter 5 - Curriculum vitae.................................................... 181

Appendix AI: Cieply B., Riley P., Pifer PM., et al., Suppression of the Epithelial-Mesenchymal Transition by Grainyhead-like-2. Cancer Research 72(9); 2440-53.

Appendix AII: Farris J., Pifer PM., et al., Grainyhead-like 2 Reverses the Metabolic Changes Induced by the Oncogenic Epithelial-mesenchymal Transition: Effects on Anoikis. Molecular Cancer Research (In Press) 


\section{LIST OF ABBREVIATIONS}

ac

acetyl-CoA

AKT

ALL

AP-1

Axd

Bd

BMP

CBP

ccRCC

ChIP

Cldn3

Cldn4

CREB

CSC

DNA

E1a

E-cadherin

EDC

EGF

EID

EMT

ENU

Ep-CAM

ERK

ESRP1/2

Etv4 acetylation

acetyl-coenzyme A

protein kinase B acute lymphoid leukemia activator protein 1

Axial defect bromodomain Bone morphogenetic protein Creb-binding protein p300 cell renal cell carcinoma Chromatin Immunoprecipitation claudin 3 claudin 4 cAMP responsive element binding protein Cancer stem cells deoxyribonucleic acids adenovirus early region $1 \mathrm{~A}$ protein epithelial-cadherin epidermal differentiation complex epidermal growth factor E1a-like inhibitor of differentiation protein epithelial-mesenchymal transition N-ethyl-N-nitrosourea epithelial cell adhesion molecule extracellular signal-regulated kinase epithelial splicing regulatory proteins 1 and 2 Ets variant 4 transcription factor 
FGFR2-IIIb

Foxj1

GDNF

GLUD1

Grh

GRHL1

GRHL2

GRHL3

H3

H4

HAT

HBE

HDAC1

HDAC2

HGF

$\mathrm{HIF} \alpha$

HIV

hTERT

IBID

ISH

K

KIX

Krt5

LBP1a

LBP-9

MAPK

MDCK

Fibroblast growth factor receptor 2-IIIb

forkhead box J1

glial cell line-derived neurotrophic factor glutamate dehydrogenase 1 grainyhead Grainyhead-like-1 Grainyhead-like-2 Grainyhead-like-3 histone 3 histone 4 histone acetyltransferase domain Human bronchial epithelial histone deacetylase 1 histone deacetylase 2 hepatocyte growth factor hypoxia-induced factor alpha human immunodeficiency virus human telomerase reverse transcriptase IRF-3 Binding Domain In Situ Hybridization lysine kinase inducible domain keratin 5 upstream binding protein 1 transcription factor CP2-like 1 mitogen-activated protein kinase Madin-Darby Canine Kidney 

mouse inner medullary collecting duct

MLL mixed lineage leukemia

MMP matrix metalloproteases

MOZ monocytic leukemia zinc finger

MSP mesenchymal subpopulation

MUC5AC

MZT

Nkx2-1

OVOL1

OVOL2

p300

p53

p63

PDGF

pEMT

PHD

PI3K

PSG

qPCR

RET

RING

RNA

RTS

Scgb1a1

shRNA

Six1 mucin 5AC, oligomeric mucus/gel-forming maternal to zygotic transition NK2 homeobox 1 ovo-like transcriptional repressor 1 ovo-like transcriptional repressor 2 E1A binding protein p300, Ep300, and KAT3B tumor protein p53 tumor protein p63 platelet-derived growth factor partial epithelial-mesenchymal transition plant homeodomain phosphatidylinositol-3-kinases penicillin-Streptomycin-Glutamine quantitative polymerase chain reaction ret proto-oncogene Really Interesting New Gene domain ribonucleic acid Rubinstein-Taybi syndrome secretoglobin family $1 \mathrm{~A}$ member 1 short hairpin RNA SIX homeobox 1transcription factor viii 
Spint1

TFCP2

TGF- $\beta$

TIC

TJP1

VHL

ZEB1 serine peptidase inhibitor Kunitz type 1 transcription factor CP2 transforming growth factor beta tumor initiating cells tight junction protein 1 Von Hippel-Lindau Zinc finger E-box-binding homeobox 1 
Chapter 1

\section{Introduction and Literature Review}




\section{Introduction to the Epithelial-Mesenchymal Transition}

\section{Epithelial Cells}

Epithelial cells enclose organs and glands forming a protective barrier by directly interacting with neighboring cells. In two dimensional culture, epithelial cells form an intact cobblestone monolayer with high transepithelial electrical resistance. In three dimensional matrices such as collagen or matrigel, epithelial cells form spherical cysts that have a distinct cell polarity. The basal side of the epithelial cell attaches to the extracellular matrix through the basement membrane, and the apical side faces the lumen of the duct where it can secrete molecules into the lumen and control the uptake of molecules. Polarity is essential for the survival of epithelial cells as detachment from the extracellular matrix leads to anoikis. Anoikis is programmed cell death caused by detachment from an extracellular matrix (Frisch and Francis, 1994). Epithelial cells form barriers through the interaction of specific epithelial proteins such as epithelial-cadherin (E-cadherin), claudins, occludins, and desmogleins (Baum and Georgiou, 2011; Turksen and Troy, 2011). E-cadherin’s extracellular domain forms a homophilic interaction with other E-cadherin molecules of adjacent cells, forming adherens junctions. The cytosolic domain of E-cadherin is important for cortical actin assembly and the sequestration of $\beta$-catenin (Qin et al., 2005; Tian et al., 2011). Claudins and occludins contribute to cell polarity by forming tight junctions which restrict integral membrane protein movement to the apical and basolateral membranes (Yeaman et al., 1999). In the cytosol, epithelial specific proteins, Rab25 and Ankyrin G, help ensure that junctional proteins are localized properly (Kizhatil et al., 2007a; Kizhatil et al., 2007b). Two major characteristics of epithelial cells are cell-cell adhesions and cell polarity. 
Our laboratory has proposed that the epithelial cell is the default cell phenotype in both development and cancer. Under this hypothesis, the epithelial phenotype is adopted automatically by a cell in the absence of genomic/epigenetic alterations or inductive differentiation signaling (Frisch, 1997). Developmentally, E-cadherin-expressing cells are the first to emerge, at the late Two-cell stage; these eventually give rise to all differentiated cell types of the adult organism (Hyafil et al., 1980; Vestweber and Kemler, 1985; Larue et al., 1994). This idea has been invoked in recent studies from other laboratories to explain, for example, the mesenchymal-epithelial transition (MET) occurring at metastatic sites (Thiery and Sleeman, 2006; Polyak and Weinberg, 2009; Tam and Weinberg, 2013). The regulation of the epithelial phenotype is important for understanding all of these processes.

\section{Mesenchymal Cells}

Mesenchymal cells lack many of the cell-cell adhesion molecules that epithelial cells express, and therefore lack significant cell-cell adhesion and polarity (Taube et al., 2010). However, mesenchymal cells express their own unique markers such as N-cadherin, fibronectin, and vimentin which are not present or have low expression in epithelial cells. Mesenchymal cells are able to migrate and invade as individual cells and can survive independent of an extracellular matrix interaction. This property makes mesenchymal cells resistant to anoikis (Frisch and Francis, 1994; Scheel et al., 2011; Frisch et al., 2013). Mesenchymal cells are essential for the formation of the extracellular matrix and may function as mesenchymal stem cells in some populations such as chondrocytes, osteoblasts, and adipocytes (Dominici et al., 2006). 


\section{Physiological Epithelial-Mesenchymal Transition}

Epithelial cells can transform into mesenchymal cells by a process referred to as the epithelial-mesenchymal transition (EMT). EMT is associated with loss of cell-cell adhesion molecules and cell polarity and gain of motility, anoikis resistance, and chemotherapeutic resistance. EMT is classified into three different types: Type 1- Developmental EMT, Type 2Fibrosis and tissue regeneration, and Type 3- Cancer progression and metastasis (Kalluri and Weinberg, 2009). During development, cells demonstrate significant plasticity by undergoing EMT and the opposite process, mesenchymal-epithelial transitions (MET). In mice, the depletion of Snail1 and Snail2, critical EMT driving transcription factors, leads to failed gastrulation because the epithelial cells are unable to EMT thereby preventing the formation of the primitive streak (Hudson et al., 2009). The reverse process, MET, is essential for neural tube formation where epithelial cells are required for neural tube closure. Depletion of Grainyheadlike-2, an epithelial regulating transcription factor, prevents neural tube closure in mice (Rifat et al.; Werth et al., 2010; Pyrgaki et al., 2011). After neural tube closure, another sequence of EMT and MET is responsible for the neural crest delamination and the formation of notochord and somites (Thiery et al., 2009).

Type 2 EMT occurs in organ fibrosis, wound healing, and tissue regeneration. Wound healing requires myofibroblasts for restructuring of the extracellular matrix and wound contractility, and keratinocytes undergo a partial EMT to form an attached epithelial sheet over a wound (Savagner et al., 2005; Thiery et al., 2009). Also, kidney epithelial cells may undergo an EMT during kidney fibrosis (Liu, 2010). 


\section{EMT in Cancer}

Type 3 EMT occurs in metastasis and cancer progression. EMT is a physiological process that is hijacked by cancer to increase survival and invasion. Type 3 EMT gives a survival advantage to cancer cells by increasing motility, invasiveness, anoikis resistance, and chemotherapeutic resistance (Kalluri and Weinberg, 2009). EMT can contribute to a chemotherapeutic resistant cancer sub-population leading to tumors that are refractory to treatment (Woodward et al., 2007; Barr et al., 2008; Li et al., 2008). These characteristics allow cancer cells to metastasize from the primary tumor site and form colonies in other parts of the body (Kalluri and Weinberg, 2009). Metastasis is responsible for the majority of cancer related deaths. Carcinomas, cancers of the epithelium, undergo multiple genetic hits leading to uncontrolled proliferation during the progression from normal epithelium to an invasive carcinoma. However, metastatic dissemination does not occur until invasive properties of the cell have been increased which are usually conferred by type 3 EMT. Cells that have undergone an EMT are known to upregulate matrix metalloproteases (MMP) which are responsible for the degradation of the extracellular matrix allowing cancer cells to invade through the basement membrane and disseminate (Ala-aho et al., 2002; Culhaci et al., 2004; Yuan et al., 2005; Park et al., 2008a; Mehner et al., 2014). After the cancer cell invades through the basement membrane, the cell can intravasate, be transported via the circulatory system to a distant site, extravasate, and establish a distant metastasis (Kalluri and Weinberg, 2009). It should be noted that for metastasis to occur, the cells must become anoikis resistance because this process requires detachment from the extracellular matrix, which under normal physiological conditions this would induce apoptosis in epithelial cells (Frisch et al., 2013). 
Mechanistically, one of the most studied phenomena of the EMT is loss of E-cadherin and its subsequent implications in cancer. Constitutive E-cadherin expression in cancer cell lines can inhibit cancer's invasive properties (Frixen et al., 1991), and E-cadherin expression has been shown to inversely predict cancer metastasis (Dorudi et al., 1993; Younis et al., 2007). Ecadherin could block the invasive traits by structurally interacting with other epithelial cells via its homophilic interaction keeping the cells in close contact and preventing invasion. Also, Ecadherin sequesters factors such as $\beta$-catenin (Wnt pathway), NRAGE (pro-apoptotic factor), SMAD3 (TGF- $\beta$ pathway), and YAP/TAZ (Hippo Signaling) on the cellular membrane preventing pathway activation, and the loss of E-cadherin can cause the activation of these respective pathways thereby promoting an EMT.

Cancer stem cells (CSC), also known as tumor initiating cells (TIC), have been shown to recapitulate tumors in a variety of different cancers such as leukemia, lymphoma, breast, and brain cancer (Bergsagel, 1967; Terpstra et al., 1996; Bonnet and Dick, 1997; Al-Hajj et al., 2003; Gupta et al., 2009b). The epithelial-mesenchymal transition can cause cells to acquire CSC properties such as the ability to form mammospheres, differentiate into two separate cell populations, and generate tumors in animals that recapitulate the heterogeneity of tumor histology (Mani et al., 2008). The clinical significance of CSC theory is that one cell can cause a tumor recurrence suggesting the eradication of all tumorigenic cells is necessary for the prevention of recurrence and metastasis. The CSC theory is still debated, and the percentage of tumor that CSCs comprise is controversial (Gupta et al., 2009a). In breast cancer research, one of the most commonly accepted CSC markers is the CD $44^{\mathrm{High} /} \mathrm{CD} 24^{\mathrm{Low}}$ phenotype. In human mammary luminal epithelial (HMLE) cells, normal epithelial cells (CD44 $\left.{ }^{\text {low }} \mathrm{CD} 24^{\text {high }}\right)$ undergo an EMT causing the cells to exhibit the $\mathrm{CD} 44^{\mathrm{High} /} \mathrm{CD} 24^{\mathrm{Low}}$ mesenchymal subpopulation 
phenotype. This conversion is associated with increased ability of the CD $44^{\mathrm{High} /} \mathrm{CD} 24^{\mathrm{Low}}$ cells to form tumors in mice (Mani et al., 2008), and this phenotype is supported by TFG- $\beta$ and Wnt autocrine and paracrine signaling (Scheel et al., 2011).

\section{Regulation of EMT}

Many EMT promoting factors have been discovered, but only a few EMT inhibitory factors have been found. Growth factors derived from the mesenchymal stroma such as EGF, HGF, PDGF, and TGF- $\beta$ can promote an EMT (Kalluri and Weinberg, 2009; Xu et al., 2009). The activation of multiple pathways such as RAS/MAPK/ERK, PI3K/AKT, $\beta$-catenin/LEF, and TGF- $\beta$ /SMAD3 (usually activated by the growth factors just described) lead to the upregulation of EMT driving transcription factors (Kalluri and Weinberg, 2009; Sanchez-Tillo et al., 2012). Two of these transcription factors, ZEB1 and Snail, are E-box binding transcription factors and function by direct repression of the E-cadherin promoter (Cano et al., 2000; Grooteclaes et al., 2003). Other EMT promoting transcription factors such as Twist, Goosecoid, and FOXC2 function more indirectly, usually upregulating ZEB1 or Snail (Thiery et al., 2009; Taube et al., 2010). These mechanisms emphasize the importance of E-cadherin downregulation for EMT to occur.

Inhibitors of EMT have remained fairly elusive. Bone morphogenetic protein-7 (BMP7), a member of the TGF- $\beta$ superfamily, can prevent EMT and cause MET by inhibiting TGF- $\beta$ signaling (Zeisberg et al., 2003). Other BMP family members are able to inhibit TGF- $\beta$ signaling and prevent EMT (Cieply et al., 2012). The mir-200 microRNA family is downregulated in EMT and when constitutively expressed, the microRNA family can suppress EMT by repressing ZEB1/2. Alternatively, ZEB1/2 are able to repress mir-200 microRNA expression thereby forming a negative feedback loop (Gregory et al., 2008; Wellner et al., 2009; Gregory et al., 
2011). The splicing regulatory proteins, epithelial splicing regulatory proteins 1 and 2 (ESRP1/2), are responsible for epithelial specific alternative splicing events in the cell such as FGFR2-IIIb, an epithelial specific FGFR2 isoform. ESRP1/2 shRNA depletion causes an EMT, and ESRP1 overexpression causes MDA-MB-231 cells to re-express epithelial alternative spliced isoforms (Warzecha et al., 2009; Warzecha et al., 2010). The first EMT inhibiting transcription factor discovered was GRHL2 which will be discussed at length in subsequent sections (Cieply et al., 2012). Another family of EMT inhibiting transcription factors is the ovo-like transcription factor family, OVOL1 and OVOL2, which function by repressing the ZEB1 promoter and upregulating ESRP1/2 (Roca et al., 2013).

Also, our previous work reported that adenovirus E1A establishes an epithelial phenotype, suppresses EMT, enhances anoikis, suppresses MMP expression through AP-1 signaling, and acts as tumor suppressor (Frisch, 1991, 1994, 1996; Somasundaram et al., 1996;

Frisch, 2001; Frisch and Mymryk, 2002). One of the major functions of E1A is its modulation of p300. A deletion of E1A n-terminus amino acids 2-36 prevents interaction with p300 and is unable to transform cells (Stein et al., 1990). Of utmost importance in this work, E1A demonstrates functional similarity with the GRHL2 which will be discussed subsequently.

\section{Epigenetics and Post-translational modifications of histones}

The blueprint of the human body is comprised of paired deoxyribonucleic acids, DNA, with a double helix structure discovered by Watson and Crick in 1953 (Watson, 1953). The DNA comprising one human genome is approximately 2 meter in length, but it fits inside almost every cell in the human body (Dancy and Cole, 2015). DNA is organized on nucleosomes, the fundamental subunit of chromatin, and is packaged into a cell's nucleus being about $10 \mu \mathrm{m}$ in diameter. A nucleosome is comprised of 146 DNA base pairs bound to a histone octamer 
containing duplicates of H2A, H2B, H3, and H4 (Dancy and Cole, 2015). Although, DNA contains the genetic information for all genes, it is becoming readily apparent that epigenetic regulation is essential for proper gene expression (Kiefer, 2007). Chromatin structure conformation is regulated by histone modifications and DNA modification which are some of the important factors that determine the "On/Off” status of the DNA/Chromatin complex. The "On” state chromatin conformation means the chromatin is open and permissive for gene expression machinery to bind and function. The "Off” state chromatin conformation means the chromatin is closed and inaccessible to gene expression machinery (Dancy and Cole, 2015). Histone 3 (H3) is highly regulated during this “On/Off” switch, and H3 is one of the proteins studied in the work presented subsequently. Post-translational modifications of H3 occur on the n-terminus of the protein, most frequently on lysine $(\mathrm{K})$ residues. Two of the most important histone modification are methylation and acetylation, but others modifications occur such as phosphorylation, deimination, ADP ribosylation, ubiquitylation, and sumoylation (Bannister and Kouzarides, 2011). Methylation of H3K4 is a signature of transcriptional activation. Monomethylation (me1) of H3K4 denotes enhancer activation and trimethylation (me3) of H3K4 denotes promoter activation (Calo and Wysocka, 2013). H3K4 methylation is mediated by the SET1/MLL histone methylase family (Yokoyama et al., 2004; Dou et al., 2005; Cho et al., 2007b). However, most other H3 methylation marks such as H3K27me3 and H3K9me3 are repressive gene signature marks (Kim and Kim, 2012). Acetylation of the H3 tail is a signature of transcriptional activation, and frequently occurs on H3K27 and H3K9 (Creyghton et al., 2010; Karmodiya et al., 2012). H3K27ac is mediated by the histone acetyltransferase, p300 (Kasper et al., 2010). H3K4 and H3K27 distinguish the enhancer activation state. The H3K4me1 and H3K27me3 histone mark is a signature of a poised but not active enhancer, and H3K4me1 and H3K27ac is a 
signature of an active enhancer (Creyghton et al., 2010). Histone modifications are highly regulated to allow epigenetic control of gene expression.

\section{Plasticity in EMT/MET}

Although EMT has been shown to increase metastasis, recent emerging studies suggest that EMT plasticity, the adaptability of a cell to change between an epithelial and a mesenchymal phenotype, is more important than the permanent conversion to mesenchymal cells for forming metastases. For example, when Twist1, an EMT promoting transcription factor, is transiently expressed, it promotes metastasis. However, constitutive Twist1 expression prevents metastasis formation (Schmidt et al., 2015). Other studies have demonstrated that a MET may enhance metastatic colonization (Kalluri and Weinberg, 2009). Conceptually, tumor cells exist as epithelial cells in the primary tumor site, when the primary site undergoes stress (hypoxia, chemotherapy, or other selective pressures), the tumor cells undergo an EMT allowing for anoikis resistance and increased invasion. The EMT tumor cells can then spread to a distant site where the tumor cell undergoes a MET and forms a distant metastasis with epithelial characteristics (Bissell et al., 2002; Jechlinger et al., 2002). The EMT-MET process is supported by the fact that breast cancer metastases express E-cadherin and demonstrate other epithelial characteristic (Kowalski et al., 2003). The EMT-MET process has two significant implications. First, the prevention of either the EMT or the MET could prevent metastasis. Secondly, the EMT cancer cells are relatively resistant to chemotherapeutic treatment making them very hard to eradicate. The two clinical solutions to this issue would be to lock the tumor cells in the epithelial state and eradicate using standard chemotherapeutic methods, or design cancer treatments that specifically kill the EMT/CSC phenotype. 
As more publications demonstrate the importance of both EMT and MET in the formation of metastases, it becomes clear that cell plasticity between the two states may be the most important factor contributing to metastatic disease. Both metastatic sites and tumor recurrences are often resistant to first line chemotherapeutic treatment because of either upregulation of compensatory signaling pathways or de novo mutations in the targeted pathway. This process is associated with cell plasticity driven by epigenetic modification machinery. Cells with this plasticity are able to survive stressors such as chemotherapy, oxidative stress, hypoxia, nutrient deficiency, and matrix detachment. Drug-resistance, tumor recurrence, and disease progression have been clearly linked to EMT and CSC (Creighton et al., 2009; Oliveras-Ferraros et al., 2012; Mitra et al., 2015; Ye and Weinberg, 2015; Marcucci et al., 2016).

Importantly, both phenotypic and epigenetic plasticity contribute to recurrence and drug resistance (Scheel and Weinberg, 2011; Singh et al., 2014; Ye and Weinberg, 2015; Doherty et al., 2016). In breast cancer, increased levels of mesenchymal circulating tumor cells correlate with cancer progression, and chemotherapy increases the shift to the mesenchymal phenotype (Yu et al., 2013). Emerging evidence suggests EMT is driven by epigenetic re-programming (Tam and Weinberg, 2013). For example, E-cadherin is epigenetically silenced by histonemodifying enzymes leading to varying degrees of repression depending on the level of plasticity needed. The histone methyltransferase Polycomb repressive complexes promote EMT by catalyzing H3K27me3 of the E-cadherin promoter leading to transcriptional repression (Bracken et al., 2006; Cao et al., 2008; Herranz et al., 2008). Histone acetylation is often is sign of transcriptional activation, and during metastasis, Snail recruits histone deacetylases, HDAC1 and HDAC2, to the E-cadherin promotor leading to silencing (Peinado et al., 2004; von Burstin et al., 2009). Clinical trials using epigenetic therapies to target drug resistant solid tumors is 
currently underway using de-acetylating and de-methylating agents (Brown et al., 2014). The ZEB1 drug-resistant phenotype, an EMT-driven cancer cell subpopulation with increased invasiveness and chemoresistance, has been reversed by using the HDAC1 inhibitor, mocetinostat (Meidhof et al., 2015; Zhang et al., 2015). Furthermore, EMT transcription factors, ZEB1 and Twist1, contribute to epigenetic modifications that occur in plasticity, the CSC phenotype, and the drug resistant phenotype (Brabletz and Brabletz, 2010; Malouf et al., 2013).

\section{Introduction to Grainyhead-like-2}

\section{Discovery of GRHL2}

The grainyhead-like transcription factor family is named for grainyhead (Grh), the Drosophila homolog. The Grh mutant causes defects in cuticle, trachea, and head formation resulting in embryonic lethality. Abnormal head granular sclerites lead to a "grainyhead" phenotype which is the namesake of the family (Nüsslein-Volhard et al.). Grh was discovered for its regulation of the Ultrabithorax and dopa decarboxylase promoters (Bray et al., 1989; Dynlacht et al., 1989). Grh interacts with the RNA polymerase II preinitiation complex for its transcriptional activity (Dynlacht et al., 1991). Grh regulates trachea elongation, post-embryonic neuroblasts, epidermal wound response, and the promoter of the Drosophila E-cadherin homolog, shotgun (Hemphälä et al., 2003; Almeida and Bray, 2005; Mace et al., 2005).

Grhl1 and Grhl2 were discovered by using Grh protein sequence in a murine GenBank protein search query (Wilanowski et al., 2002), and Grhl3 was discovered by querying similar protein sequences to Grhl1 and Grhl2’s dimerization domain (Ting et al., 2003b). Human GRHL2 has 94\% homology with murine Grhl2. The grainyhead-like transcription factors are part of the LSF/GRH transcription factor family. Phylogenetic analysis suggests two distinct 
branches of this family, the GRH subfamily consists of GRHL1, GRHL2, and GRHL3, and the LSF/CP2 subfamily consists of TFCP2, LBP1a, and LBP-9 transcription factors (Wilanowski et al., 2002; Traylor-Knowles et al., 2010). The structure of GRHL1, 2, and 3 contain an Nterminal transcriptional activation domain, a central DNA binding immunoglobulin fold domain similar to p53, and a C-terminal dimerization domain which is predicted to have ubiquitin-like folds (Kokoszynska et al., 2008). The GRHL proteins are able to homo- and hetero-dimerize with each other, but they are not able to dimerize with other LSF/GRH transcription factors (Wilanowski et al., 2002).

In murine studies, Grhl2 is observed at high expression levels in the primitive surface ectoderm from E8.5 until E12.5 when the expression starts to decline until E15.5. At this time, Grhl2 is only present in basal layer, stratum spinosum and stratum granulosum of the ectoderm. During murine development, Grhl2 is present in kidney, lungs, olfactory epithelium, esophagus, stomach, small and large intestine, urogenital tract, oral epithelium, and submandibular gland. Interestingly, Grhl2 expression is inversely expressed in a spatial and temporal manner compared to Grhl1 and Grhl3 in the developing lung and kidney (Auden et al., 2006).

\section{Function of GRHL2 in epidermis}

The role of GRHL2 has been intensively studied in the epidermis and associated skin disorders. In keratinocytes, GRHL2 increases cell replicative life span by increasing telomerase activity via upregulation of the hTERT promoter. GRHL2 knockdown decreases cell proliferation, reduces epithelial thickness in three dimensional culture, and causes hypermethylation of the hTERT promoter indicating that GRHL2 partially regulates hTERT through an epigenetic mechanism (Chen et al., 2010b). Importantly, GRHL2 is required for the upregulation of E-cadherin, claudin 4, and claudin 3, epithelial apical junction complex proteins 
(Werth et al., 2010; Senga et al., 2012). Grhl2 binds the Cldn4 promoter and the second intronic region of E-cadherin increasing promoter activity in both cases (Werth et al., 2010). Since GRHL2 appears to be the master regulator of the epithelial phenotype, it is not surprising that GRHL2 correlates with E-cadherin expression better than any other transcription factor (Werth et al., 2010; Kohn et al., 2014), and Grhl2/-- mice show a reduction in E-cadherin and Cldn4 expression (Werth et al., 2010). GRHL2 also suppresses ZEB1 and upregulates mir-200c and ESRP1/2 (Cieply et al., 2012; Cieply et al., 2013). Mir-200c is a microRNA associated with the establishment of the epithelial phenotype by suppressing ZEB1 expression (Park et al., 2008b). ESRP1 and ESRP2 are responsible for promoting epithelial specific RNA splicing of multiple transcripts (Warzecha et al., 2009). In keratinocytes, GRHL2 and p63, a member of the p53 family, form a positive feedback loop which is necessary for proper epidermal development, and the loss of p63 causes an EMT (Truong et al., 2006; Mehrazarin et al., 2015).

Both Grhl1/- and Grhl3/-/ mice show epidermal defects (Boglev et al., 2011), however, embryonic lethality of Grhl2-/- mice at E11.5 precludes the examination of epidermal defects. $\mathrm{Grhl2}^{-/+} / \mathrm{Grhl}^{-/+}$mice have defects in epithelial wound repair. Furthermore, GRHL2 $2^{-/+}$GRHL3I- mice have failure of eyelid closure and are born with open eyes. This suggests a defect in epithelial cell migration in utero (Boglev et al., 2011).

GRHL2 prevents keratinocyte terminal differentiation by repressing the epidermal differentiation complex (EDC). GRHL2 blocks the recruitment of Jmjd3, a histone demethylase, to the EDC gene promoters (Chen et al., 2012). GRHL2 is also overexpressed in hyperproliferative skin lesions seen in psoriatic tissue (Chen et al., 2012). A GRHL2 mutation causes an autosomal-recessive ectodermal dysplasia syndrome in two consanguineous families presenting with hypodontia, enamel hypoplasia, oral hyperpigmentation, nail dystrophy, 
marginal palmoplantar keratodema, and dysphagia. Also, a fraction of the patients present with sensorineural deafness. When the patient's keratinocytes were cultured, the cells had decreased epithelial features including defective epithelial adhesion junction complexes, altered cell morphology, mis-localizition of GRHL2 in the cytoplasm, and decreased E-cadherin expression (Petrof et al., 2014).

\section{Function of GRHL2 in neural tube closure}

Both Grhl2 and Grhl3 are involved in the neural tube closure. In the murine model, primary neurulation occurs at three separate closure sites; hindbrain/cervical (closure 1), forebrain/midbrain (closure 2), and rostral end of the brain (closure 3). For neural closure to transpire, neural fold elevation must occur in conjunction with non-neural ectodermal-induced formation of paired dorso-lateral hinge points (Shum and Copp, 1996). Grhl2-/- mice exhibit failure of the rostral end of the forebrain closure (closure 3) leading to exencephaly and a splitface malformation. Grhl2/-- mice fail to produce paired dorso-lateral hinges at which point primary neurulation ceases and defects are observed (Rifat et al.), and these results were confirmed in subsequent GRHL2 mouse models (Werth et al., 2010; Pyrgaki et al., 2011). $\mathrm{Grhl}^{-/-}$mice produce thoraco-lumbo-sacral spina bifida, but only exhibit exencephaly in $3 \%$ of the mice (Ting et al., 2003a), and Grhl2 substitution into Grhl3 genome loci did not rescue the spina bifida phenotype suggesting non-redundant functions of Grhl2 and Grhl3. Interestingly, $\mathrm{Grhl}^{-/ /} / \mathrm{Grhl}^{+/-}$mice displayed failed forebrain/midbrain closure (closure 2) causing fully penetrant exencephaly, and there was no defect in hindbrain/cervical closure (closure 1). This data suggest that closure 3 is dependent on Grhl2, closure 2 is dependent on total Grhl2 and Grhl3 dose, and closure 1 is independent of $G r h l$ genes (Rifat et al.). Axial defect, Axd, mice present with spina bifida caused by the upregulation of GRHL2, and GRHL2 downregulation in 
Axd mice prevented the spina bifida (Brouns et al., 2011). Since both the upregulation and downregulation of GRHL2 causes neural tube defects, the expression levels of GRHL2 is critical for proper development. An ENU-induced GRHL2 nonsense mutation results in the previous published GRHL2 neural tube defects and previously unobserved defects; thoracoabdominoschisis, failure of optic fissure closure, and heart and lung defects (Pyrgaki et al., 2011). The GRHL2 nonsense mutation causes down-regulation of multiple epithelial genes including E-cadherin, desmoglein 2, Ep-CAM, ESRP1, and CLDN4 (Pyrgaki et al., 2011). This GRHL2 nonsense mutation causes the non-neural ectoderm to exhibit a mesenchymal phenotype leading to the failure of cranial neural fold elevation ultimately causing the GRHL2-associated neural tube defects. The Centers for Disease Control recommends all women with the possibility of pregnancy to take folic acid to prevent spina bifida (CDC 1992). Interestingly, folic acid supplementation increases spina bifida in $\mathrm{Grhl}^{+/-}$mice (Marean et al., 2011). However, GRHL2 has never been implicated in folic acid metabolism. Since folic acid has been demonstrated to cause epigenetic changes (Li et al., 2011a), it could be downregulating GRHL2 leading to the increase in spina bifida incidence.

\section{Function of GRHL2 in liver}

GRHL2 promotes liver cholangiocytes, but not hepatocytes. Mature cholangiocyte cells form larger cysts in three dimensional culture than liver progenitor cells. By comparing transcription factors differentially regulated between hepatoblasts and cholangiocytes, GRHL2 is upregulated in the mature cholangiocytes, and subsequently when expressed in liver progenitor cells causes the formation of larger cysts by increasing luminal space. In two dimensional culture, GRHL2 increases transepithelial electric resistance when expressed in the liver progenitor cells (Senga et al., 2012). GRHL2 upregulates CLDN3 and CLDN4 which are 
important for establishing epithelial cell barrier function. Expression of CLDN3, but not CLDN4, is able to increase cyst lumen size in the liver progenitor cells (Senga et al., 2012). Rab25, a vesicle transport protein, increases cyst size and regulates CLDN4 expression and localization at cell-cell contacts and tight junctions. These results were the first to demonstrate the role of GRHL2 on tight junctions and regulation of cell polarity, and its importance in cholangiocytes (Tanimizu et al., 2013). During development, neonatal cholangiocytes are able to differentiate into hepatocytes and express low levels of GRHL2. However, adult cholangiocytes express high levels of GRHL2 and cannot differentiate into hepatocytes (Tanimizu et al., 2013). The overexpression of GRHL2 in neonatal cholangiocytes prevents hepatocyte differentiation, and reciprocally, the knockdown of GRHL2 in adult cholangiocytes allows these cells to differentiate into hepatocytes (Tanimizu et al., 2013). Mir-122 correlates with hepatocyte differentiation (Laudadio et al.), and GRHL2 binds and represses the Mir-122 promoter (Tanimizu et al., 2014). GRHL2 is responsible for maintaining the cholangiocyte phenotype and preventing hepatocyte differentiation.

\section{Function of GRHL2 in lung}

Drosophila Grh expression finely regulates tracheal elongation and apical membrane growth. Grh depletion results in increased apical membrane growth and longer trachea whereas Grh overexpression results in decreased apical membrane growth and shorter trachea. FGF signaling inhibits GRH during apical expansion and elongation (Hemphälä et al., 2003). In the murine model, Grhl2 is expressed in luminal and basal $\mathrm{p}^{+} / \mathrm{Krt}^{+}$epithelial cells of the distal lung epithelium. However, Grhl1 and Grhl3 are expressed in the proximal lung epithelium with Grhl1 being expressed in MUC5AC ${ }^{+}$goblet cells and Grhl3 being expressed in acetylated tubulin ciliated cells (Varma et al., 2012; Gao et al., 2013). Grhl2 expression co-localizes with 
Nkx2-1 in alveolar epithelial type II cells. Nkx2-1 is a homeodomain-containing transcription factor essential for lung differentiation that regulates the E-cadherin promoter (Saito et al., 2009; Das et al., 2011). Grhl2 depletion decreases Nkx2-1 expression, and Nkx2-1 knockdown decreases Grhl2 expression. Conversely, Grhl2 binds the Nkx2-1 promoter, and Nkx2-1 binds the Grhl2 intronic region forming a positive feedback loop (Varma et al., 2012). GRHL2 establishes cuboidal morphology of alveolar type II epithelial cells, and its depletion causes the flattening of the cells which resemble alveolar type 1 epithelial cells (Varma et al., 2012).

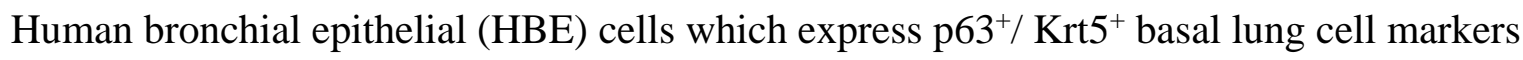
differentiate into mucociliary epithelium without losing GRHL2 expression when cultured on an air-liquid interface. In HBE cells, a GRHL2 dominant negative protein lacking the transactivation domain downregulates E-cadherin, TJP1, and CLDN4 and causes the loss of transepithelial resistance (Gao et al., 2013). Mucociliary cells are comprised of Foxj1 ${ }^{+}$ multiciliated cells, Scgb1a1 ${ }^{+}$secretory cells, and $\mathrm{p}^{+} 3^{+} \mathrm{Krt5}^{+}$basal cells, and Grhl2 is expressed in all three subtypes. When Grhl2 is depleted in the progenitor $\mathrm{Krt5} 5^{+} / \mathrm{p} 63^{+}$basal cells, there is a decrease in Foxj $1^{+}$multiciliated cells suggesting Grhl2 regulates the differentiation of multiciliated cells via a Notch3-dependent pathway (Gao et al., 2015). Grhl2 may play a role in idiopathic pulmonary fibrosis where it is downregulated, and lung epithelial cells may undergo cell flattening contributing to lung fibrosis (Varma et al., 2014).

\section{Function of GRHL2 in kidney and placental tubulogenesis}

Grhl2 is present in developing kidney ureteric buds and collecting duct epithelia (Werth et al., 2010; Aue et al., 2015). Grhl2 ${ }^{-/}$mice demonstrate decreased collecting duct luminal expansion, and Grhl2 depletion in mouse inner medullary collecting duct (miMCD) cells decreases luminal expansion in three dimensional culture and affects epithelial barrier formation 
(Aue et al., 2015). Interestingly, Grhl2 binds and upregulates OVOL2, an epithelial transcription factor (Roca et al., 2013), which is able to rescue the epithelial barrier and luminal expansion defects in miMCD cells (Aue et al., 2015).

OVOL2 controls mammary tubulogenesis by regulating the epithelial-mesenchymal transition in mammary terminal end buds (Watanabe et al., 2014), presumably downstream of GRHL2 regulation. The depletion of OVOL2 in mammary epithelial cells causes an EMT, depletes stem cells reserves, and reduces alveologenesis and milk production (Watanabe et al., 2014). Similar to GRHL2, OVOL2 reverts MDA-MB-231 cells back to an epithelial phenotype (Cieply et al., 2012; Watanabe et al., 2014).

GRHL2 also regulates placental branching morphogenesis where it is expressed in the basal chorionic trophoblast cells (Walentin et al., 2015). GRHL2 knockdown leads to the loss of basal chorionic trophoblast cell polarity and defects in labyrinth branching morphogenesis. GRHL2 upregulates Spint1, serine peptidase inhibitor Kunitz type 1, which regulates placental development (Szabo et al., 2006).

\section{Function of GRHL2 in cancer}

Our laboratory was the first to demonstrate that GRHL2 is a tumor suppressor in the claudin-low breast cancer subtype (Cieply et al., 2012; Cieply et al., 2013). Multiple other publications suggest GRHL2 can act as either a tumor suppressor or an oncogene. The mechanism of GRHL2 on cancer will be presented here to clarify its effects.

GRHL2 expression is lost in claudin-low and basal B subtypes of breast cancer which exhibit an aggressive EMT phenotype (Cieply et al., 2012). Constitutive GRHL2 expression causes a MET in the MDA-MB-231 breast cancer cell line and the HMLE mesenchymal 
subpopulation (MSP) cell line. In breast, ovarian, and colorectal cancers, GRHL2 and ZEB1 have been shown to form a negative feedback loop where GRHL2 either directly or indirectly represses the ZEB1 promoter, and ZEB1 directly represses the GRHL2 promoter (Cieply et al., 2013; Werner et al., 2013; Quan et al., 2014; Chung et al., 2016). Furthermore, GRHL2 prevents Six1, a developmental EMT homeoprotein transcription factor, from transactivating the ZEB1 promoter (Cieply et al., 2013). GRHL2 inhibits TGF- $\beta$ signaling by inhibiting SMAD transcription (Cieply et al., 2012). GRHL2 also upregulates mir-200a, mir-200b, OVOL1/2 and ESRP1/2 (see EMT for discussion) (Cieply et al., 2012; Chen et al., 2016). In anoikis resistant MDA-MB-231 cells and HMLE MSP lines, GRHL2 restores anoikis sensitivity (Cieply et al., 2012; Farris et al., 2016). The anoikis sensitizing effect of GRHL2 is partially regulated through its downregulation of glutamate dehydrogenase 1 (GLUD1), a mitochondrial enzyme that produces $\alpha$-ketoglutarate. GLUD1 promotes tumorigenicity in an orthotopic cancer model and correlates with advanced stages of breast and lung cancers (Jin et al., 2015). GRHL2 represses the GLUD1 promoter through a p300-dependent mechanism (Pifer et al., 2016).

Upon paclitaxol treatment, GRHL2 suppresses the emergence of the CSC population in HMLE and MDA-MB-231 cell lines (Cieply et al., 2013). This suggests that GRHL2 locks cells into an epithelial state preventing the emergence of CSCs thereby inhibiting cancer recurrence. In a murine model of drug resistant recurrent tumors, GRHL2 is downregulated in recurrent tumors compared to primary tumors (Cieply et al., 2012). In another study, epithelial SUM149 breast cancer cells were deprived of glutamine until a metabolically adaptable cell population emerged that is resistant to multiple chemotherapeutics and downregulates GRHL2 (Singh et al., 2014). The metabolically adaptable cell population exhibits reduction in histone activation 
marks, H3K4 trimethylation and H3K14 acetylation, which have been previously published to correlate with a drug resistant state (Sharma et al., 2010).

Werner et al. demonstrate a loss of GRHL2 at the invasive front of breast cancer tumor samples, and GRHL2 inversely correlates with lymph node metastasis and higher tumor staging (Werner et al., 2013). The study also anecdotally notes that basal cell carcinoma and noninvasive bladder cancer have high GRHL2 expression and very rarely metastasize (Werner et al., 2013). In ovarian cancer cells, GRHL2 depletion enhances invasion (Chung et al., 2016). This information suggests how the loss of GRHL2 can lead to an EMT and increase in metastasis.

However, GRHL2 has also been reported to be associated with poor relapse-free survival and metastasis in human breast cancer (Xiang et al., 2012; Yang et al., 2013b). In Xiang et al, GRHL2 knockdown was confirmed to produce an EMT in human mammary epithelial cells. However, GRHL2 overexpression in 4T1 murine mammary carcinoma cells causes an increase in lung metastasis. One explanation for this observation is 4T1 cells with GRHL2 form larger primary tumors thereby increasing tumor shedding. Interestingly, GRHL2 is downregulated in the metastasis of the parental 4T1 cells arguing against GRHL2's metastasis enhancing properties (Xiang et al., 2012). In oral squamous cell carcinoma, GRHL2 binds and upregulated the hTERT promoter increasing proliferation and promoting tumorigenicity (Kang et al., 2008; Chen et al., 2010b). GRHL2 is amplified in hepatocellular carcinoma and predicts early recurrence (Tanaka et al., 2008). In colorectal carcinomas, GRHL2 expression enhances proliferation and correlates with larger tumor size and advanced staging (Quan et al., 2014; Quan et al., 2015). In gastric cancer, an increase in GRHL2 copy number has been demonstrated (Cheng et al., 2012). However, a subsequent study shows GRHL2 is downregulated in gastric 
cancer and constitutive GRHL2 expression decreases proliferation and enhances apoptosis (Xiang et al., 2013).

The role of GRHL2 appears to be dependent upon cancer type. However, it is very clear that GRHL2 maintains or establishes the epithelial phenotype in these cancers. This is usually associated with chemotherapeutic susceptibility (See EMT section), and therefore the GRHL2expressing cells should be more responsive to classical chemotherapeutic treatment.

\section{p300}

\section{Discovery of p300/CBP}

p300 and Creb binding protein (CBP) are highly homologous transcriptional co-activators that interact with DNA-binding transcription factors on an extraordinary amount of promoters. p300 (Ep300,KAT3B) and CBP (Crebbp, KAT3A) form the KAT3 histone acetyltransferase family sharing five similar protein interaction domains discussed subsequently (Arany et al., 1995; Lundblad et al., 1995). p300 was discovered from its interaction with E1a which modulates P300 HAT activity and function (noted previously) (Eckner et al., 1994; Bannister et al., 1995; Goodman and Smolik, 2000; Santer et al., 2011). p300"/- mice die at E9.5-11.5 from defects in neurulation and heart development and decreased proliferation of mesenchymal cells, erythroid cells, and colony forming cells (Yao et al.; Oike et al., 1999). CBP was discovered from its association with CREB transcription factor (Chrivia et al., 1993). Because p300/CBP are highly redundant, p300/CBP will be referred to as p300 unless specific situations are being discussed where the proteins display different functions (Kalkhoven, 2004). 


\section{Structure of $p 300$}

p300 is a 300kDa protein with 2014 amino acids, and its various domains interact with over 400 proteins (Bedford et al., 2010). The domains and structure of p300 are shown in figure 1. The nuclear receptor interaction domain (RID) is important for binding of nuclear hormone receptors. p300 has three cysteine/histidine regions, CH1(TAZ1), CH2 (RING and PHD), and CH3(ZZ and TAZ2) which each contain two zinc binding motifs with four alpha helices (De Guzman et al., 2005a). CH1(TAZ1) and (CH3)TAZ2 regions are responsible for interaction with multiple transcription factors such as HIF1, p73, twist, c-fos, and ets-1 (Frisch and Mymryk, 2002; De Guzman et al., 2005b). The CH2 domain contains two functional domains, PHD and RING. The plant homeodomain (PHD) domain is a cysteine-histidine-cysteine motif found in many acetyltransferases (Bordoli et al., 2001). RING (Really Interesting New Gene) domains are normally present in ubiquitin E3 ligases. However in p300, the RING domain inhibits the active site of the p300's histone acetyltransferase domain (HAT) forming an autoinhibitory loop (Delvecchio et al., 2013; Ringel and Wolberger, 2013). Interestingly, p300 mutations that cause cancer occur in this RING region (Delvecchio et al., 2013). The kinase inducible domain (KIX) is a three helix bundle that interacts with CREB and other transcription factors (Chrivia et al., 1993; Thakur et al., 2013). The bromodomain (Bd) is responsible for binding acetyl-lysine residues on histones and transcription factors (Chen et al., 2010a). This domain is responsible for forming a stable interaction with chromatin allowing transcriptional activation to occur (Manning et al., 2001). The histone acetyltransferase region (HAT) is the catalytic region for acetylation, and it will be discussed in greater detail in the p300 function section. p300 C-terminal domain (1665aa-2414aa) can cause transcriptional transactivation, and its effects are potentiated by the p300 HAT (Stiehl et al., 2007). The minimal domain for p300 
C-terminal transactivation is 2000-2180 aa which includes the IRF-3 binding domain (IBID) also known as the SRC1 interaction domain (SID) (2050-2096aa) (Lin et al., 2001; Matsuda et al., 2004). The p300 IBID domain cooperates with SRC1, a p160/steroid receptor coactivator family member, to activate transcription (Sheppard et al., 2001).

\section{Functions of p300}

p300 facilitates transcriptional activation by the acetylation of histone tails and/or transcription factors via its intrinsic histone/lysine acetyltransferase activity (HAT/KAT)(Ogryzko et al., 1996). Histone acetylation causes relaxation of chromatin structure allowing increase accessibility for transcriptional machinery. In vitro, p300 is able to acetylate all four core histones (Ogryzko et al., 1996). In p300-/- mice, histone 3 lysine 18 and 27 acetylation is lost demonstrating p300's specificity for these substrates (Kasper et al., 2010; Jin et al., 2011). p300 also acetylates transcription factors leading to regulation of their function. p300 acetylates p53 in response to many stressors causing an increase in p53 DNA binding and

protein stability (Gu and Roeder, 1997; Knights et al., 2006). p300 acetylates $\beta$-catenin causing nuclear localization and increases affinity for TCF4 (Lévy et al., 2004; Chocarro-Calvo et al., 2013).

p300 may function as a signal integrator because of the dose limiting amount of p300 in the cell, and the multitude of signaling pathways and cellular processes that require p300 for transcriptional activation (Yao et al.,1998; Kamei et al., 1996; Vo and Goodman, 2001; Bedford et al., 2010). This is further supported by the fact that a single mutation in CBP allele leads to Rubinstein-Taybi syndrome (discussed later) suggesting the protein level of p300 is very important (Petrif et al., 1995). Also, enhanceosomes, a group of proteins that bind and recruit co-activators and basal transcription factors to promoters, in some instances contain multiple 
transcription factors that bind p300 such as the IFN- $\beta$ enhanceosomes (Merika et al., 1998; Yie et al., 1999)

p300 has been demonstrated to be essential for the differentiation of muscle, erythroid lineages, osteoblast, oligodendrocytes, and stem cells (Puri et al., 1997; Oike et al., 1999; Blobel, 2000; Polesskaya et al., 2001; Narayanan et al., 2004; Teo and Kahn, 2010; Zhang et al., 2016). In a similar process to cell differentiation, activator proteins that utilize p300 and additional coactivators that interact with p300 have been implicated in the EMT phenotype and tumor progression (Matthews et al., 2007; Qin et al., 2009; Santer et al., 2011; Zhou et al., 2012; Delvecchio et al., 2013; Ringel and Wolberger, 2013; Yang et al., 2013a; Xu et al., 2014; Cho et al., 2015). The upregulation of matrix metalloproteases (MMPs) is associated with the EMT phenotype and contributes to tumor invasion (Radisky and Radisky, 2010). Many MMPS are regulated through p300 and the AP-1 transcription factor axis (Westermarck and Kahari, 1999; Sun et al., 2004; Chou et al., 2006; Clark et al., 2008; Lee and Partridge, 2010; Santer et al., 2011).

Although the role of p300 in the acetylation of histone has been well researched, the effect of p300 on other histone modifications is emerging. Recent results demonstrate p300 interacts with the SET1 complex through a p53 mediated event to regulate H3K4 trimethylation (Tang et al., 2013). The SET1/MLL family (SET1A, SET1B, SET1C, MLL1, MLL2, MLL3 and MLL4) is a group of histone methyltransferases responsible for H3K4 monomethylation and trimethylation (Yokoyama et al., 2004; Dou et al., 2005; Cho et al., 2007a; Wu et al., 2008). The SET1/MLL members are part of the Set1/MLL/COMPASS-like complex which include Wdr5, Ash2, RbBp5, and other proteins depending upon which Set1/MLL family member is present in the complex (Shilatifard, 2012). H3K4 trimethylation is a necessary, specific marker 
for active gene transcription on eukaryotic promoters (Santos-Rosa et al., 2002; Ng et al., 2003). In the p53/p300/SET1C interaction, p53 recruits p300 to chromatin, and then p300 acetylates H3 and binds the acetyl-lysine residues via p300's bromodomain stabilizing the interaction. Both p53 and p300 directly interact with SET1C and enhance the H3K4 trimethylation of the promoter. The p53/p300/SET1C complex promotes H3 acetylation and methylation thereby increasing transcription (Tang et al., 2013). Interestingly, a recent study has demonstrated p300 is a marker for super-enhancers that place it as a position to control cell state transitions (Witte et al., 2015).

\section{p300/CBP in disease and cancer}

The loss of one allele of CBP and less frequently, p300, leads to Rubinstein-Taybi syndrome (RTS) (Petrif et al., 1995). RTS occurs in about one in 100,000 live births from mostly spontaneous mutations (Roelfsema et al., 2005). The causative mutation of RTS can be located in multiple exons and consist of deletions, insertions, or point mutations in CBP/p300 (Roelfsema et al., 2005; Roelfsema and Peters, 2007; Viosca et al., 2010). RTS is characterized by dysmorhphic facial features (low hairline, low set ears, arched eyebrows, slanted palpebral fissures, arched palate, mild micrognathia. and dental anomalies), enlarged first finger, cervical vertebral abnormalities, and RTS patients are at higher risk for sensorineural deafness, optic nerve coloboma, enamel hypoplasia, and congenital heart and renal defects (Petrif et al., 1995). Specifically relating to cancer, RTS patients are at increased risk of neuroblastoma, medulloblastoma, leukemia and lymphoma (Siraganian et al., 1989; Skousen et al., 1996; de Kort et al., 2014). More generally, p300 has been implicated and discussed as a therapeutic target for HIV retroviral infections, platelet cell production, heart disease, and diabetes (Gusterson et al., 2003; Zhou et al., 2004; Kauppi et al., 2008; Mujtaba and Zhou, 2011). In 
these processes, p300 is vital for transcriptional regulation, and inhibition could have therapeutic advantages.

p300 exhibits both tumor suppressor and oncogenic functions depending on the context it is examined (Goodman and Smolik, 2000; Wang et al., 2013; Dancy and Cole, 2015). As discussed previously, RTS patients have increased risk of malignancies during their life. Multiple studies have shown the loss of heterozygosity of p300 and/or CBP in cancer cell lines and ovarian, colon, and breast cancer samples (Muraoka et al., 1996; Bryan et al., 2002; Tillinghast et al., 2003). p300 mutations have been demonstrated in ovarian, pancreatic, cervical, colorectal, and oral squamous tumors or tumor cell lines (Iyer et al., 2001). Also, CBP loss of heterozygosity has been shown to correlate with therapy resistance in acute lymphoblastic leukemia (ALL) (Mullighan et al., 2011). On a molecular level, p300 interacts with and promotes the function of known tumor suppressors, p53 and retinoblastoma protein (Gu and Roeder, 1997; Grossman, 2001; Iyer et al., 2007).

On the other hand, p300 overexpression has also been correlated with cancer progression. In nasopharyngeal carcinoma, hepatocellular carcinomas, and prostate cancer, increased p300 levels lead to poor prognosis and increased aggressiveness of the cancer (Debes et al., 2003; Li et al., 2011b; Santer et al., 2011; Liao et al., 2012). In breast cancer, p300 is overexpressed in invasive ductal carcinomas (Hudelist et al., 2003; Vleugel et al., 2006). In melanoma, cytoplasmic localization instead of nuclear localization of p300 is associated with aggressiveness and poor prognosis (Rotte et al., 2013). p300/CBP can also fuse with the mixed lineage leukemia (MLL) and monocytic leukemia zinc finger (MOZ) genes to form an oncogenic fusion protein and target p300 acetyltransferase activity to oncogenic genes in ALL (Borrow et al., 1996; Ida et al., 1997; Taki et al., 1997). On a molecular level, p300 is involved in the 
transcriptional machinery of multiple oncogenic proteins including AP-1 complex (c-jun and cfos), c-myb, and human T-cell leukemia virus (Arias et al., 1994; Bannister et al., 1995; Dai et al., 1996; Zhao et al., 2011). As discussed previously, p300 is very context dependent in its role in cancer. Because p300/CBP is a global transcriptional activator that can interact with both oncogenic and tumor suppressors, these effects should not come as a surprise.

\section{Inhibitors of p300 HAT Function}

The HAT domain of p300 is responsible for the acetylation of proteins. The p300 acetyltransferase domain uses acetyl-CoA and a lysine containing protein (usually histones) to form an acetyl-lysine-containing protein and coenzyme A with a sulfhydryl functional group (Dancy and Cole, 2015). The acetylation step occurs in the HAT active site containing six acidic residues, and the lysine residue of the protein to be acetylated is usually surrounded by basic amino acids (Liu et al., 2008). The acetyltransferase reaction is predicted to occur through a Theorell-Chance, hit-and-run catalytic, mechanism which involves no stable ternary complex and transient peptide substrate association (Cleland, 1963; Liu et al., 2008).

Lys-CoA, a bisubstrate of acetyl-lysine and coenzyme A, was the first potent acetyltransferase inhibitor identified and works by interacting with both individual substrate sites of p300, simultaneously (Lau et al., 2000). Lys-CoA is highly specific for p300/CBP, but LysCoA and its derivatives are not cell permeable making it ill-suited for therapeutics (Dancy and Cole, 2015). Many natural chemicals have been suggested as acetyltransferase inhibitors. Anacardic acid, a salicylic acid with an alkyl chain, is derived from geraniums, cashew shells, and mango and has broad effects on all KAT families (Balasubramanyam et al., 2003; Ghizzoni et al., 2012). Curcumin, two phenols connected by two carbonyls, is derived from turmeric and has acetyltransferase inhibitor affects in the p300/CBP and MYST HAT families 
(Balasubramanyam et al., 2004b). Garcinol, a polyisoprenylated benzophenone, is derived for Garcinia indica and is a potent inhibitor of p300 and PCAF. However, Garcinol is cytotoxic, but some of its derivatives show promise (Balasubramanyam et al., 2004a). Other natural HAT inhibitor have been discovered, but they have broad specificity and only modest potency to all the HAT families which chemical modifications have not improved (Varier et al., 2004; Choi et al., 2009).

Since 2005, synthetic inhibitors for p300 HAT activity have been emerging. Isothazolone analogs were demonstrated to inhibit acetyltransferases, but these chemicals inhibited PCAF more potently than p300 (Stimson et al., 2005; Dekker et al., 2009). Quinolone compounds also inhibit acetyltransferases and some of these analogs have modest potency for CBP and p300 (Mai et al., 2006; Mai et al., 2009). The most promising compound, C646, has three aromatic rings and a benzoic acid and is a potent inhibitor of p300/CBP with great specificity over other HAT families (Bowers et al., 2010). C646 appears to compete for the acetyl-CoA binding site and not the peptide substrate, and C646 has inhibitory effects on acetylation in cells (Santer et al., 2011).

Although more than four hundred proteins interact with p300 and utilize it as a co-activator, the inhibitory effect of GRHL2 on p300 is nearly unique, shared by only one other family of proteins called the E1a-like inhibitor of differentiation (EID) proteins. EID1 inhibits p300 HAT activity (MacLellan et al., 2000) and EID3 blocks the SRC-1/CBP interaction, while the mechanism of EID2 is controversial (Ji et al., 2003; Miyake et al., 2003; Båvner et al., 2005). The effects of EID proteins upon EMT or tubulogenesis have not yet been investigated to our knowledge. 


\section{MDCK tubulogenesis}

The opposing roles of GRHL2 in establishing the epithelial state vs. p300 in differentiation -including EMT -- motivated us to investigate a potential role for GRHL2 in inhibiting p300 function. Kidney tubulogenesis is contingent upon temporal and spatial regulation of epithelial cells in which GRHL2 plays a critical organizing role. Furthermore, GRHL2 is expressed in the ureteric buds of the developing kidney (Schmidt-Ott et al., 2005; Aue et al., 2015; Walentin et al., 2015). Madin-Darby Canine Kidney (MDCK) tubulogenesis in response to HGF models some aspects of kidney collecting duct tubulogenesis in vivo and requires a transient, partial EMT (pEMT) (Pollack et al., 1998; O'Brien et al., 2002; Pollack et al., 2004; Leroy and Mostov, 2007; Hellman et al., 2008; Jung et al., 2012; Zhang et al., 2014). Kidney development involves multiple signaling pathways (GDNF, FGFs, BMP4, HGF, and Gremlin) with glial cell linederived neurotrophic factor (GDNF) and its receptor, RET, being the most important (Costantini and Kopan, 2010). However, RET receptor is not expressed in MDCK cells. HGF causes MDCK cells to undergo cell scattering in two-dimensional culture, and induces tubulogenesis of MDCK cysts that form in a three-dimensional collagen gel (Wang et al., 1990, Pollack et al., 2004). Induction of MMP1 and MMP13 by HGF through AP1/p300 complexes is required for

tubulogenesis in the MDCK model (Benbow and Brinckerhoff, 1997; Clark et al., 2008; Hellman et al., 2008; Chacon-Heszele et al., 2014). Targeted knockout studies indicate a role for HGF/Met signaling in kidney development, and HGF/Met signaling is a potent stimulator of Wnt signaling, a crucial signaling pathway in kidney development, validating the in vivo relevance of this approach (Monga et al., 2002; Bridgewater et al., 2008; Ishibe et al., 2009). 


\section{References}

Al-Hajj, M., Wicha, M., Benito-Hernandez, A., Morrison, S., and Clarke, M. (2003). Prospective identification of tumorigenic breast cancer cells. Proc Natl Acad Sci U S A early edition, 1-6.

Ala-aho, R., Johansson, N., Baker, A.H., and Kähäri, V.-M. (2002). Expression of collagenase-3 (MMP-13) enhances invasion of human fibrosarcoma HT-1080 cells. Int J Cancer 97, 283-289.

Almeida, M.S., and Bray, S.J. (2005). Regulation of post-embryonic neuroblasts by Drosophila Grainyhead. Mech Dev 122, 1282-1293.

Arany, Z., Newsome, D., Oldread, E., Livingston, D.M., and Eckner, R. (1995). A family of transcriptional adaptor proteins targeted by the E1A oncoprotein. Nature 374, 81-84.

Arias, J., Alberts, A.S., Brindle, P., Claret, F.X., Smeal, T., Karin, M., Feramisco, J., and Montminy, M. (1994). Activation of cAMP and mitogen responsive genes relies on a common nuclear factor. Nature 370, 226-229.

Auden, A., Caddy, J., Wilanowski, T., Ting, S.B., Cunningham, J.M., and Jane, S.M. (2006). Spatial and temporal expression of the Grainyhead-like transcription factor family during murine development. Gene Expr Patterns 6, 964-970.

Aue, A., Hinze, C., Walentin, K., Ruffert, J., Yurtdas, Y., Werth, M., Chen, W., Rabien, A., Kilic, E., Schulzke, J.-D., Schumann, M., and Schmidt-Ott, K.M. (2015a). A Grainyhead-Like 2/Ovo-Like 2 Pathway Regulates Renal Epithelial Barrier Function and Lumen Expansion. JASN 26, 2704-2715.

Balasubramanyam, K., Altaf, M., Varier, R.A., Swaminathan, V., Ravindran, A., Sadhale, P.P., and Kundu, T.K. (2004a). Polyisoprenylated Benzophenone, Garcinol, a Natural Histone Acetyltransferase Inhibitor, Represses Chromatin Transcription and Alters Global Gene Expression. J Biol Chem 279, 33716-33726.

Balasubramanyam, K., Swaminathan, V., Ranganathan, A., and Kundu, T.K. (2003). Small Molecule Modulators of Histone Acetyltransferase p300. J Biol Chem 278, 19134-19140.

Balasubramanyam, K., Varier, R.A., Altaf, M., Swaminathan, V., Siddappa, N.B., Ranga, U., and Kundu, T.K. (2004b). Curcumin, a Novel p300/CREB-binding Protein-specific Inhibitor of Acetyltransferase, Represses the Acetylation of Histone/Nonhistone Proteins and Histone Acetyltransferase-dependent Chromatin Transcription. J Biol Chem 279, 51163-51171. 
Bannister, A.J., and Kouzarides, T. (2011). Regulation of chromatin by histone modifications. Cell Res 21, 381-395.

Bannister, A.J., Oehler, T., Wilhelm, D., Angel, P., and Kouzarides, T. (1995). Stimulation of cJun activity by CBP: c-Jun residues Ser63/73 are required for CBP induced stimulation in vivo and CBP binding in vitro. Oncogene 11, 2509-2514.

Barr, S., Thomson, S., Buck, E., Russo, S., Petti, F., Sujka-Kwok, I., Eyzaguirre, A., RosenfeldFranklin, M., Gibson, N.W., Miglarese, M., Epstein, D., Iwata, K.K., and Haley, J.D. (2008). Bypassing cellular EGF receptor dependence through epithelial-to-mesenchymal-like transitions. Clin Exp Metastasis 25, 685-693.

Baum, B., and Georgiou, M. (2011). Dynamics of adherens junctions in epithelial establishment, maintenance, and remodeling. J Cell Biology 192, 907-917.

Båvner, A., Matthews, J., Sanyal, S., Gustafsson, J.-Å., and Treuter, E. (2005). EID3 is a novel EID family member and an inhibitor of CBP-dependent co-activation. Nucleic Acids Res 33, 3561-3569.

Bedford, D.C., Kasper, L.H., Fukuyama, T., and Brindle, P.K. (2010). Target gene context influences the transcriptional requirement for the KAT3 family of CBP and p300 histone acetyltransferases. Epigenetics 5, 9-15.

Benbow, U., and Brinckerhoff, C.E. (1997). The AP-1 site and MMP gene regulation: What is all the fuss about? Matrix Biology 15, 519-526.

Bergsagel, D.E. (1967). The Chronic Leukemias: A Review of Disease Manifestations and the Aims of Therapy. CMAJ 96, 1615-1620.

Bissell, M.J., Radisky, D.C., Rizki, A., Weaver, V.M., and Petersen, O.W. (2002). The organizing principle: microenvironmental influences in the normal and malignant breast. Differentiation; 70, 537-546.

Blobel, G.A. (2000). CREB-binding protein and p300: molecular integrators of hematopoietic transcription. Blood 95, 745-755.

Boglev, Y., Wilanowski, T., Caddy, J., Parekh, V., Auden, A., Darido, C., Hislop, N.R., Cangkrama, M., Ting, S.B., and Jane, S.M. (2011). The unique and cooperative roles of the 
Grainy head-like transcription factors in epidermal development reflect unexpected target gene specificity. Dev Biol 349, 512-522.

Bonnet, D., and Dick, J.E. (1997). Human acute myeloid leukemia is organized as a hierarchy that originates from a primitive hematopoietic cell. Nat Med 3, 730-737.

Bordoli, L., Hüsser, S., Lüthi, U., Netsch, M., Osmani, H., and Eckner, R. (2001). Functional analysis of the p300 acetyltransferase domain: the PHD finger of p300 but not of CBP is dispensable for enzymatic activity. Nucleic Acids Res 29, 4462-4471.

Borrow, J., Stanton, V.P., Andresen, J.M., Becher, R., Behm, F.G., Chaganti, R.S.K., Civin, C.I., Disteche, C., Dube, I., Frischauf, A.M., Horsman, D., Mitelman, F., Volinia, S., Watmore, A.E., and Housman, D.E. (1996). The translocation t(8;16)(p11;p13) of acute myeloid leukaemia fuses a putative acetyltransferase to the CREB-binding protein. Nat Genet 14, 33-41.

Bowers, E.M., Yan, G., Mukherjee, C., Orry, A., Wang, L., Holbert, M.A., Crump, N.T., Hazzalin, C.A., Liszczak, G., Yuan, H., Larocca, C., Saldanha, S.A., Abagyan, R., Sun, Y., Meyers, D.J., Marmorstein, R., Mahadevan, L.C., Alani, R.M., and Cole, P.A. (2010). Virtual Ligand Screening of the p300/CBP Histone Acetyltransferase: Identification of a Selective Small Molecule Inhibitor. Chemistry \& Biology 17, 471-482.

Brabletz, S., and Brabletz, T. (2010). The ZEB/miR-200 feedback loop-a motor of cellular plasticity in development and cancer? EMBO reports 11, 670-677.

Bracken, A.P., Dietrich, N., Pasini, D., Hansen, K.H., and Helin, K. (2006). Genome-wide mapping of Polycomb target genes unravels their roles in cell fate transitions. Genes Dev. 20, 1123-1136.

Bray, S.J., Burke, B., Brown, N.H., and Hirsh, J. (1989). Embryonic expression pattern of a family of Drosophila proteins that interact with a central nervous system regulatory element. Genes Dev. 3, 1130-1145.

Bridgewater, D., Cox, B., Cain, J., Lau, A., Athaide, V., Gill, P.S., Kuure, S., Sainio, K., and Rosenblum, N.D. (2008). Canonical WNT/ $\beta$-catenin signaling is required for ureteric branching. Dev Biol 317, 83-94.

Brouns, M.R., De Castro, S.C.P., Terwindt-Rouwenhorst, E.A., Massa, V., Hekking, J.W., Hirst, C.S., Savery, D., Munts, C., Partridge, D., Lamers, W., Köhler, E., van Straaten, H.W., Copp, A.J., and Greene, N.D.E. (2011). Over-expression of Grhl2 causes spina bifida in the Axial defects mutant mouse. Hum Mol Gen 20, 1536-1546. 
Brown, R., Curry, E., Magnani, L., Wilhelm-Benartzi, C.S., and Borley, J. (2014). Poised epigenetic states and acquired drug resistance in cancer. Nat Rev Cancer 14, 747-753.

Bryan, E.J., Jokubaitis, V.J., Chamberlain, N.L., Baxter, S.W., Dawson, E., Choong, D.Y.H., and Campbell, I.G. (2002). Mutation analysis of EP300 in colon, breast and ovarian carcinomas. Int J Cancer 102, 137-141.

Calo, E., and Wysocka, J. (2013). Modification of Enhancer Chromatin: What, How, and Why? Molecular Cell 49, 825-837.

Cano, A., Perez-Moreno, M.A., Rodrigo, I., Locascio, A., Blanco, M.J., del Barrio, M.G., Portillo, F., and Nieto, M.A. (2000). The transcription factor snail controls epithelialmesenchymal transitions by repressing E-cadherin expression. Nat Cell Biol 2, 76-83.

Cao, Q., Yu, J., Dhanasekaran, S.M., Kim, J.H., Mani, R.S., Tomlins, S.A., Mehra, R., Laxman, B., Cao, X., Yu, J., Kleer, C.G., Varambally, S., and Chinnaiyan, A.M. (2008). Repression of Ecadherin by the polycomb group protein EZH2 in cancer. Oncogene 27, 7274-7284.

Center for Disease control and Prevention (1992). Recommendations for the use of folic acid to reduce the number of cases of spina bifida and other neural tube defects. MMWR.

Recommendations And Reports: Morbidity And Mortality Weekly Report. Recommendations And Reports / Centers For Disease Control 41, 1-7.

Chacon-Heszele, M.F., Zuo, X., Hellman, N.E., McKenna, S., Choi, S.Y., Huang, L., Tobias, J.W., Park, K.M., and Lipschutz, J.H. (2014). Novel MAPK-dependent and -independent tubulogenes identified via microarray analysis of 3D-cultured Madin-Darby canine kidney cells. JASN 306, F1047-F1058.

Chen, J., Ghazawi, F.M., and Li, Q. (2010a). Interplay of bromodomain and histone acetylation in the regulation of p300-dependent genes. Epigenetics 5, 509-515.

Chen, W., Dong, Q., Shin, K.-H., Kim, R.H., Oh, J.-E., Park, N.-H., and Kang, M.K. (2010b). Grainyhead-like 2 Enhances the Human Telomerase Reverse Transcriptase Gene Expression by Inhibiting DNA Methylation at the 5'-CpG Island in Normal Human Keratinocytes. J Biol Chem 285, 40852-40863.

Chen, W., Xiao Liu, Z., Oh, J.E., Shin, K.H., Kim, R.H., Jiang, M., Park, N.H., and Kang, M.K. (2012). Grainyhead-like 2 (GRHL2) inhibits keratinocyte differentiation through epigenetic mechanism. Cell Death \& Disease 3, e450. 
Chen, W., Yi, J.K., Shimane, T., Mehrazarin, S., Lin, Y.-L., Shin, K.-H., Kim, R.H., Park, N.-H., and Kang, M.K. (2016). Grainyhead-like 2 regulates epithelial plasticity and stemness in oral cancer cells. Carcinogenesis.

Cheng, L., Wang, P., Yang, S., Yang, Y., Zhang, Q., Zhang, W., Xiao, H., Gao, H., and Zhang, Q. (2012). Identification of genes with a correlation between copy number and expression in gastric cancer. BMC Medical Genomics 5, 14-14.

Cho, M.H., Park, J.H., Choi, H.J., Park, M.K., Won, H.Y., Park, Y.J., Lee, C.H., Oh, S.H., Song, Y.S., Kim, H.S., Oh, Y.H., Lee, J.Y., and Kong, G. (2015). DOT1L cooperates with the c-Mycp300 complex to epigenetically derepress CDH1 transcription factors in breast cancer progression. Nat Commun 6, 7821.

Cho, Y.-W., Hong, T., Hong, S., Guo, H., Yu, H., Kim, D., Guszczynski, T., Dressler, G.R., Copeland, T.D., Kalkum, M., and Ge, K. (2007a). PTIP Associates with MLL3- and MLL4containing Histone H3 Lysine 4 Methyltransferase Complex. J Biol Chem 282, 20395-20406.

Cho, Y.W., Hong, T., Hong, S., Guo, H., Yu, H., Kim, D., Guszczynski, T., Dressler, G.R., Copeland, T.D., Kalkum, M., and Ge, K. (2007b). PTIP associates with MLL3- and MLL4containing histone H3 lysine 4 methyltransferase complex. J Biol Chem 282, 20395-20406.

Chocarro-Calvo, A., García-Martínez, Jose M., Ardila-González, S., De la Vieja, A., and GarcíaJiménez, C. (2013). Glucose-Induced $\beta$-Catenin Acetylation Enhances Wnt Signaling in Cancer. Molecular Cell 49, 474-486.

Choi, K.-C., Jung, M.G., Lee, Y.-H., Yoon, J.C., Kwon, S.H., Kang, H.-B., Kim, M.-J., Cha, J.H., Kim, Y.J., Jun, W.J., Lee, J.M., and Yoon, H.-G. (2009). Epigallocatechin-3-Gallate, a Histone Acetyltransferase Inhibitor, Inhibits EBV-Induced B Lymphocyte Transformation via Suppression of RelA Acetylation. Cancer Res 69, 583-592.

Chou, Y.T., Wang, H., Chen, Y., Danielpour, D., and Yang, Y.C. (2006). Cited2 modulates TGF-[beta]-mediated upregulation of MMP9. Oncogene 25, 5547-5560.

Chrivia, J.C., Kwok, R.P.S., Lamb, N., Hagiwara, M., Montminy, M.R., and Goodman, R.H. (1993). Phosphorylated CREB binds specifically to the nuclear protein CBP. Nature 365, 855859.

Chung, V.Y., Tan, T.Z., Tan, M., Wong, M.K., Kuay, K.T., Yang, Z., Ye, J., Muller, J., Koh, C.M., Guccione, E., Thiery, J.P., and Huang, R.Y.-J. (2016). GRHL2-miR-200-ZEB1 maintains 
the epithelial status of ovarian cancer through transcriptional regulation and histone modification. Scientific Reports 6, 19943.

Cieply, B., Farris, J., Denvir, J., Ford, H.L., and Frisch, S.M. (2013). Epithelial-Mesenchymal Transition and Tumor Suppression Are Controlled by a Reciprocal Feedback Loop between ZEB1 and Grainyhead-like-2. Cancer Res 73, 6299-6309.

Cieply, B., Riley, P.t., Pifer, P.M., Widmeyer, J., Addison, J.B., Ivanov, A.V., Denvir, J., and Frisch, S.M. (2012). Suppression of the Epithelial-Mesenchymal Transition by Grainyhead-like2. Cancer Res 72, 2440-2453.

Clark, I.M., Swingler, T.E., Sampieri, C.L., and Edwards, D.R. (2008). The regulation of matrix metalloproteinases and their inhibitors. Int J Biochem Cell 40, 1362-1378.

Cleland, W.W. (1963). The kinetics of enzyme-catalyzed reactions with two or more substrates or products. Biochim Biophys Acta 67, 188-196.

Costantini, F., and Kopan, R. (2010). Patterning a complex organ: branching morphogenesis and nephron segmentation in kidney development. Developmental cell 18, 698-712.

Creighton, C.J., Li, X., Landis, M., Dixon, J.M., Neumeister, V.M., Sjolund, A., Rimm, D.L., Wong, H., Rodriguez, A., Herschkowitz, J.I., Fan, C., Zhang, X., He, X., Pavlick, A., Gutierrez, M.C., Renshaw, L., Larionov, A.A., Faratian, D., Hilsenbeck, S.G., Perou, C.M., Lewis, M.T., Rosen, J.M., and Chang, J.C. (2009). Residual breast cancers after conventional therapy display mesenchymal as well as tumor-initiating features. Proc Natl Acad Sci U S A 106, 13820-13825.

Creyghton, M.P., Cheng, A.W., Welstead, G.G., Kooistra, T., Carey, B.W., Steine, E.J., Hanna, J., Lodato, M.A., Frampton, G.M., and Sharp, P.A. (2010a). Histone H3K27ac separates active from poised enhancers and predicts developmental state. Proc Natl Acad Sci U S A 107.

Culhaci, N., Metin, K., Copcu, E., and Dikicioglu, E. (2004). Elevated expression of MMP-13 and TIMP-1 in head and neck squamous cell carcinomas may reflect increased tumor invasiveness. BMC Cancer 4, 42-42.

Dai, P., Akimaru, H., Tanaka, Y., Hou, D.X., Yasukawa, T., Kanei-Ishii, C., Takahashi, T., and Ishii, S. (1996). CBP as a transcriptional coactivator of c-Myb. Genes Dev 10, 528-540.

Dancy, B.M., and Cole, P.A. (2015). Protein lysine acetylation by p300/CBP. Chemical Reviews 115, 2419-2452. 
Das, A., Acharya, S., Gottipati, K.R., McKnight, J.B., Chandru, H., Alcorn, J.L., and Boggaram, V. (2011). Thyroid transcription factor-1 (TTF-1) gene: identification of ZBP-89, Sp1, and TTF1 sites in the promoter and regulation by TNF- $\alpha$ in lung epithelial cells. Am J Physiol Lung Cell Mol Physiol 301, L427-L440.

De Guzman, R.N., Martinez-Yamout, M.A., Dyson, H.J., and Wright, P.E. (2005a). Structure and Function of the CBP/p300 TAZ Domains. In: Zinc Finger Proteins: From Atomic Contact to Cellular Function, AM J Physiol Lung Cell Mol, 114-120.

De Guzman, R.N., Wojciak, J.M., Martinez-Yamout, M.A., Dyson, H.J., and Wright, P.E. (2005b). CBP/p300 TAZ1 Domain Forms a Structured Scaffold for Ligand Binding. Biochemistry 44, 490-497.

de Kort, E., Conneman, N., and Diderich, K. (2014). A case of Rubinstein-Taybi syndrome and congenital neuroblastoma. Am. J. Med. Genet 164, 1332-1333.

Debes, J.D., Sebo, T.J., Lohse, C.M., Murphy, L.M., Haugen, D.A.L., and Tindall, D.J. (2003). p300 in prostate cancer proliferation and progression. Cancer Res63, 7638-7640.

Dekker, F.J., Ghizzoni, M., van der Meer, N., Wisastra, R., and Haisma, H.J. (2009). Inhibition of the PCAF histone acetyl transferase and cell proliferation by isothiazolones. Bioorg Med Chem Lett. 17, 460-466.

Delvecchio, M., Gaucher, J., Aguilar-Gurrieri, C., Ortega, E., and Panne, D. (2013). Structure of the p300 catalytic core and implications for chromatin targeting and HAT regulation. Nat Struct Mol Biol 20, 1040-1046.

Doherty, R.M., Smigiel, M.J., Junk, J.D., and Jackson, W.M. (2016). Cancer Stem Cell Plasticity Drives Therapeutic Resistance. Cancers 8.

Dominici, M., Le Blanc, K., Mueller, I., Slaper-Cortenbach, I., Marini, F., Krause, D., Deans, R., Keating, A., Prockop, D., and Horwitz, E. (2006). Minimal criteria for defining multipotent mesenchymal stromal cells. The International Society for Cellular Therapy position statement. Cytotherapy 8, 315-317.

Dorudi, S., Sheffield, J.P., Poulsom, R., Northover, J.M., and Hart, I.R. (1993). E-cadherin expression in colorectal cancer. An immunocytochemical and in situ hybridization study. Am J Pathol. 142, 981-986. 
Dou, Y., Milne, T.A., Tackett, A.J., Smith, E.R., Fukuda, A., Wysocka, J., Allis, C.D., Chait, B.T., Hess, J.L., and Roeder, R.G. (2005). Physical Association and Coordinate Function of the H3 K4 Methyltransferase MLL1 and the H4 K16 Acetyltransferase MOF. Cell 121, 873-885.

Dynlacht, B.D., Attardi, L.D., Admon, A., Freeman, M., and Tjian, R. (1989). Functional analysis of NTF-1, a developmentally regulated Drosophila transcription factor that binds neuronal cis elements. Genes Dev 3, 1677-1688.

Dynlacht, B.D., Hoey, T., and Tjian, R. (1991). Isolation of coactivators associated with the TATA-binding protein that mediate transcriptional activation. Cell 66, 563-576.

Eckner, R., Arany, Z., Ewen, M., Sellers, W., and Livingston, D.M. (1994). The Adenovirus E1A-associated 300-kD Protein Exhibits Properties of a Transcriptional Coactivator and Belongs to an Evolutionarily Conserved Family. Cold Spring Harbor Symposia on Quantitative Biology 59, 85-95.

Farris, J.C., Pifer, P.M., Zheng, L., Gottlieb, E., Denvir, J., and Frisch, S.M. (2016). Grainyheadlike 2 Reverses the Metabolic Changes Induced by the Oncogenic Epithelial-mesenchymal Transition: Effects on Anoikis. Mol Cancer Res 14, 528-38

Frisch, S.M. (1991). Antioncogenic effect of adenovirus E1A in human tumor cells. Proc Natl Acad Sci U S A 88, 9077-9081.

Frisch, S.M. (1994). E1a induces the expression of epithelial characteristics. J Cell Biol 127, 1085-1096.

Frisch, S.M. (1996). Reversal of malignancy by the adenovirus E1a gene. Mutat Res 350, 261266.

Frisch, S.M. (1997). The epithelial cell default-phenotype hypothesis and its implications for cancer. Bioessays 19, 705-709.

Frisch, S.M. (2001). Tumor suppression activity of adenovirus E1a protein: anoikis and the epithelial phenotype. Adv Cancer Res 80, 39-49.

Frisch, S.M., and Francis, H. (1994). Disruption of epithelial cell-matrix interactions induces apoptosis. J Cell Biol 124, 619-626. 
Frisch, S.M., and Mymryk, J.S. (2002). Adenovirus-5 E1A: paradox and paradigm. Nat Rev Mol Cell Biol 3, 441-452.

Frisch, S.M., Schaller, M., and Cieply, B. (2013). Mechanisms that link the oncogenic epithelialmesenchymal transition to suppression of anoikis. J Cell Sci 1, 21-29

Frixen, U.H., Behrens, J., Sachs, M., Eberle, G., Voss, B., Warda, A., Löchner, D., and Birchmeier, W. (1991). E-cadherin-mediated cell-cell adhesion prevents invasiveness of human carcinoma cells. J Cell Biol 113, 173-185.

Gao, X., Bali, A.S., Randell, S.H., and Hogan, B.L.M. (2015). GRHL2 coordinates regeneration of a polarized mucociliary epithelium from basal stem cells. J Cell Biol 211, 669-682.

Gao, X., Vockley, C.M., Pauli, F., Newberry, K.M., Xue, Y., Randell, S.H., Reddy, T.E., and Hogan, B.L. (2013). Evidence for multiple roles for grainyhead-like 2 in the establishment and maintenance of human mucociliary airway epithelium.[corrected]. Proc Natl Acad Sci U S A 110, 9356-9361.

Ghizzoni, M., Wu, J., Gao, T., Haisma, H.J., Dekker, F.J., and George Zheng, Y. (2012). 6alkylsalicylates are selective Tip60 inhibitors and target the acetyl-CoA binding site. Eur J Med Chem 47, 337-344.

Goodman, R.H., and Smolik, S. (2000). CBP/p300 in cell growth, transformation, and development. Genes Dev 14, 1553-1577.

Gregory, P.A., Bert, A.G., Paterson, E.L., Barry, S.C., Tsykin, A., Farshid, G., Vadas, M.A., Khew-Goodall, Y., and Goodall, G.J. (2008). The miR-200 family and miR-205 regulate epithelial to mesenchymal transition by targeting ZEB1 and SIP1. Nat Cell Biol 10, 593-601.

Gregory, P.A., Bracken, C.P., Smith, E., Bert, A.G., Wright, J.A., Roslan, S., Morris, M., Wyatt, L., Farshid, G., Lim, Y.Y., Lindeman, G.J., Shannon, M.F., Drew, P.A., Khew-Goodall, Y., and Goodall, G.J. (2011). An autocrine TGF-beta/ZEB/miR-200 signaling network regulates establishment and maintenance of epithelial-mesenchymal transition. Mol Biol Cell 22, 16861698.

Grooteclaes, M., Deveraux, Q., Hildebrand, J., Zhang, Q., Goodman, R.H., and Frisch, S.M. (2003). C-terminal-binding protein corepresses epithelial and proapoptotic gene expression programs. Proc Natl Acad Sci U S A 100, 4568-4573. 
Grossman, S.R. (2001). p300/CBP/p53 interaction and regulation of the p53 response. Eur J Med Chem 268, 2773-2778.

Gu, W., and Roeder, R.G. (1997). Activation of p53 Sequence-Specific DNA Binding by Acetylation of the p53 C-Terminal Domain. Cell 90, 595-606.

Gupta, P., Onder, T., Jiang, G., Tao, K., Kuperwasser, C., Weinberg, R., and Lander, E. (2009a). Identification of selective inhibitors of cancer stem cells by high-throughput screening. Cell 138.

Gupta, P.B., Chaffer, C.L., and Weinberg, R.A. (2009b). Cancer stem cells: mirage or reality? Nat Med 15, 1010-1012.

Gusterson, R.J., Jazrawi, E., Adcock, I.M., and Latchman, D.S. (2003). The transcriptional coactivators CREB-binding protein (CBP) and p300 play a critical role in cardiac hypertrophy that is dependent on their histone acetyltransferase activity. J Biol Chem 278, 6838-6847.

Hellman, N.E., Spector, J., Robinson, J., Zuo, X., Saunier, S., Antignac, C., Tobias, J.W., and Lipschutz, J.H. (2008). Matrix Metalloproteinase 13 (MMP13) and Tissue Inhibitor of Matrix Metalloproteinase 1 (TIMP1), Regulated by the MAPK Pathway, Are Both Necessary for Madin-Darby Canine Kidney Tubulogenesis. J Biol Chem 283, 4272-4282.

Hemphälä, J., Uv, A., Cantera, R., Bray, S., and Samakovlis, C. (2003). Grainy head controls apical membrane growth and tube elongation in response to Branchless/FGF signalling. Development 130, 249-258.

Herranz, N., Pasini, D., Díaz, V.M., Francí, C., Gutierrez, A., Dave, N., Escrivà, M., HernandezMuñoz, I., Di Croce, L., Helin, K., García de Herreros, A., and Peiró, S. (2008). Polycomb Complex 2 Is Required for E-cadherin Repression by the Snail1 Transcription Factor. Mol Biol Cell. 28, 4772-4781.

Hudelist, G., Czerwenka, K., Kubista, E., Marton, E., Pischinger, K., and Singer, C.F. (2003). Expression of Sex Steroid Receptors and their Co-Factors in Normal and Malignant Breast Tissue: AIB1 is a Carcinoma-Specific Co-Activator. Breast Cancer Res Treat 78, 193-204.

Hudson, L.G., Newkirk, K.M., Chandler, H.L., Choi, C., Fossey, S.L., Parent, A.E., and Kusewitt, D.F. (2009). Cutaneous wound reepithelialization is compromised in mice lacking functional Slug (Snai2). J Dermatol Sci. 56, 19-26. 
Hyafil, F., Morello, D., Babinet, C., and Jacob, F. (1980). A cell surface glycoprotein involved in the compaction of embryonal carcinoma cells and cleavage stage embryos. Cell 21, 927-934.

Ida, K., Kitabayashi, I., Taki, T., Taniwaki, M., Noro, K., Yamamoto, M., Ohki, M., and Hayashi, Y. (1997). Adenoviral E1A-Associated Protein p300 Is Involved in Acute Myeloid Leukemia With t(11; 22)(q23; q13). Blood 90, 4699-4704.

Ishibe, S., Karihaloo, A., Ma, H., Zhang, J., Marlier, A., Mitobe, M., Togawa, A., Schmitt, R., Czyczk, J., Kashgarian, M., Geller, D.S., Thorgeirsson, S.S., and Cantley, L.G. (2009). Met and the epidermal growth factor receptor act cooperatively to regulate final nephron number and maintain collecting duct morphology. Development 136, 337-345.

Iyer, N.G., Ozdag, H., and Caldas, C. (2001). p300//CBP and cancer. Oncogene 23, 4225-4231.

Iyer, N.G., Xian, J., Chin, S.F., Bannister, A.J., Daigo, Y., Aparicio, S., Kouzarides, T., and Caldas, C. (2007). p300 is required for orderly G1/S transition in human cancer cells. Oncogene 26, 21-29.

Jechlinger, M., Grünert, S., and Beug, H. (2002). Mechanisms in epithelial plasticity and metastasis: insights from 3D cultures and expression profiling. J Mammary Gland Biol Neoplasia 7, 415-432.

Ji, A., Dao, D., Chen, J., and MacLellan, W.R. (2003). EID-2, a novel member of the EID family of p300-binding proteins inhibits transactivation by MyoD. Gene 318, 35-43.

Jin, L., Li, D., Alesi, Gina N., Fan, J., Kang, H.-B., Lu, Z., Boggon, Titus J., Jin, P., Yi, H., Wright, Elizabeth R., Duong, D., Seyfried, Nicholas T., Egnatchik, R., DeBerardinis, Ralph J., Magliocca, Kelly R., He, C., Arellano, Martha L., Khoury, Hanna J., Shin, Dong M., Khuri, Fadlo R., and Kang, S. (2015). Glutamate Dehydrogenase 1 Signals through Antioxidant Glutathione Peroxidase 1 to Regulate Redox Homeostasis and Tumor Growth. Cancer Cell 27, 257-270.

Jin, Q., Yu, L.-R., Wang, L., Zhang, Z., Kasper, L.H., Lee, J.-E., Wang, C., Brindle, P.K., Dent, S.Y.R., and Ge, K. (2011). Distinct roles of GCN5/PCAF-mediated H3K9ac and CBP/p300mediated H3K18/27ac in nuclear receptor transactivation. The EMBO Journal 30, 249-262.

Jung, Y.S., Liu, X.-W., Chirco, R., Warner, R.B., Fridman, R., and Kim, H.-R.C. (2012). TIMP1 Induces an EMT-Like Phenotypic Conversion in MDCK Cells Independent of Its MMPInhibitory Domain. PLoS ONE 7, e38773. 
Kalkhoven, E. (2004). CBP and p300: HATs for different occasions. Biochemical Pharmacology 68, 1145-1155.

Kalluri, R., and Weinberg, R.A. (2009). The basics of epithelial-mesenchymal transition. J Clin Invest 119, 1420-1428.

Kamei, Y., Xu, L., Heinzel, T., Torchia, J., Kurokawa, R., Gloss, B., Lin, S.-C., Heyman, R.A., Rose, D.W., Glass, C.K., and Rosenfeld, M.G. (1996). A CBP Integrator Complex Mediates Transcriptional Activation and AP-1 Inhibition by Nuclear Receptors. Cell 85, 403-414.

Kang, X., Chen, W., Kim, R.H., Kang, M.K., and Park, N.H. (2008). Regulation of the hTERT promoter activity by MSH2, the hnRNPs K and D, and GRHL2 in human oral squamous cell carcinoma cells. Oncogene 28, 565-574.

Karmodiya, K., Krebs, A.R., Oulad-Abdelghani, M., Kimura, H., and Tora, L. (2012). H3K9 and H3K14 acetylation co-occur at many gene regulatory elements, while H3K14ac marks a subset of inactive inducible promoters in mouse embryonic stem cells. BMC Genomics 13, 1-18.

Kasper, L.H., Lerach, S., Wang, J., Wu, S., Jeevan, T., and Brindle, P.K. (2010). CBP/p300 double null cells reveal effect of coactivator level and diversity on CREB transactivation. The EMBO Journal 29, 3660-3672.

Kauppi, M., Murphy, J.M., de Graaf, C.A., Hyland, C.D., Greig, K.T., Metcalf, D., Hilton, A.A., Nicola, N.A., Kile, B.T., Hilton, D.J., and Alexander, W.S. (2008). Point mutation in the gene encoding p300 suppresses thrombocytopenia in Mpl-/- mice. Blood 112, 3148-3153.

Kiefer, J.C. (2007). Epigenetics in development. Dev Dyn 236, 1144-1156.

Kim, J., and Kim, H. (2012). Recruitment and Biological Consequences of Histone Modification of H3K27me3 and H3K9me3. ILAR Journal 53, 232-239.

Kizhatil, K., Davis, J.Q., Davis, L., Hoffman, J., Hogan, B.L., and Bennett, V. (2007a). Ankyrin$\mathrm{G}$ is a molecular partner of E-cadherin in epithelial cells and early embryos. J Biol Chem 282, 26552-26561.

Kizhatil, K., Yoon, W., Mohler, P.J., Davis, L.H., Hoffman, J.A., and Bennett, V. (2007b). Ankyrin-G and beta2-spectrin collaborate in biogenesis of lateral membrane of human bronchial epithelial cells. J Biol Chem 282, 2029-2037. 
Knights, C.D., Catania, J., Giovanni, S.D., Muratoglu, S., Perez, R., Swartzbeck, A., Quong, A.A., Zhang, X., Beerman, T., Pestell, R.G., and Avantaggiati, M.L. (2006). Distinct p53 acetylation cassettes differentially influence gene-expression patterns and cell fate. J Cell Biol 173, 533-544.

Kohn, K.W., Zeeberg, B.M., Reinhold, W.C., and Pommier, Y. (2014). Gene expression correlations in human cancer cell lines define molecular interaction networks for epithelial phenotype. PLoS One 9, e99269.

Kokoszynska, K., Ostrowski, J., Rychlewski, L., and Wyrwicz, L.S. (2008). The fold recognition of CP2 transcription factors gives new insights into the function and evolution of tumor suppressor protein p53. Cell Cycle 7, 2907-2915.

Kowalski, P.J., Rubin, M.A., and Kleer, C.G. (2003). E-cadherin expression in primary carcinomas of the breast and its distant metastases. Breast Cancer Res 5, R217-R222.

Larue, L., Ohsugi, M., Hirchenhain, J., and Kemler, R. (1994). E-cadherin null mutant embryos fail to form a trophectoderm epithelium. Proc Natl Acad Sci USA 91, 8263-8267.

Lau, O.D., Kundu, T.K., Soccio, R.E., Ait-Si-Ali, S., Khalil, E.M., Vassilev, A., Wolffe, A.P., Nakatani, Y., Roeder, R.G., and Cole, P.A. (2000). HATs off: Selective Synthetic Inhibitors of the Histone Acetyltransferases p300 and PCAF. Molecular Cell 5, 589-595.

Laudadio, I., Manfroid, I., Achouri, Y., Schmidt, D., Wilson, M.D., Cordi, S., Thorrez, L., Knoops, L., Jacquemin, P., Schuit, F., Pierreux, C.E., Odom, D.T., Peers, B., and Lemaigre, F.P. A Feedback Loop Between the Liver-Enriched Transcription Factor Network and Mir-122 Controls Hepatocyte Differentiation. Gastroenterology 142, 119-129.

Lee, M., and Partridge, N.C. (2010). Parathyroid Hormone Activation of Matrix Metalloproteinase-13 Transcription Requires the Histone Acetyltransferase Activity of p300 and PCAF and p300-dependent Acetylation of PCAF. J Biol Chem 285, 38014-38022.

Leroy, P., and Mostov, K.E. (2007). Slug Is Required for Cell Survival during Partial EpithelialMesenchymal Transition of HGF-induced Tubulogenesis. Mol Bio Cell 18, 1943-1952.

Lévy, L., Wei, Y., Labalette, C., Wu, Y., Renard, C.-A., Buendia, M.A., and Neuveut, C. (2004). Acetylation of $\beta$-Catenin by p300 Regulates $\beta$-Catenin-Tcf4 Interaction. Mol. Cell. Biol. 24, 3404-3414. 
Li, C.C.Y., Cropley, J.E., Cowley, M.J., Preiss, T., Martin, D.I.K., and Suter, C.M. (2011a). A Sustained Dietary Change Increases Epigenetic Variation in Isogenic Mice. PLoS Genet 7, e1001380.

Li, M., Luo, R.-Z., Chen, J.-W., Cao, Y., Lu, J.-B., He, J.-H., Wu, Q.-L., and Cai, M.-Y. (2011b). High expression of transcriptional coactivator p300 correlates with aggressive features and poor prognosis of hepatocellular carcinoma. J. Transl. Med. 9, 1-11.

Li, X., Lewis, M.T., Huang, J., Gutierrez, C., Osborne, C.K., Wu, M.F., Hilsenbeck, S.G., Pavlick, A., Zhang, X., Chamness, G.C., Wong, H., Rosen, J., and Chang, J.C. (2008). Intrinsic resistance of tumorigenic breast cancer cells to chemotherapy. J Natl Cancer Inst 100, 672-679.

Liao, Z.-W., Zhou, T.-C., Tan, X.-J., Song, X.-L., Liu, Y., Shi, X.-Y., Huang, W.-J., Du, L.-L., Tu, B.-J., and Lin, X.-d. (2012). High expression of p300 is linked to aggressive features and poor prognosis of Nasopharyngeal Carcinoma. J. Transl. Med. 10, 1-8.

Lin, C.H., Hare, B.J., Wagner, G., Harrison, S.C., Maniatis, T., and Fraenkel, E. (2001). A Small Domain of CBP/p300 Binds Diverse Proteins: Solution Structure and Functional Studies. Mol Cell 8, 581-590.

Liu, X., Wang, L., Zhao, K., Thompson, P.R., Hwang, Y., Marmorstein, R., and Cole, P.A. (2008). The structural basis of protein acetylation by the p300/CBP transcriptional coactivator. Nature 451, 846-850.

Liu, Y. (2010). New Insights into Epithelial-Mesenchymal Transition in Kidney Fibrosis. J. AM. Soc. Nephrol. 21, 212-222.

Lundblad, J.R., Kwok, R.P.S., Laurance, M.E., Harter, M.L., and Goodman, R.H. (1995). Adenoviral ElA-associated protein p300 as a functional homologue of the transcriptional coactivator CBP. Nature 374, 85-88.

Mace, K.A., Pearson, J.C., and McGinnis, W. (2005). An Epidermal Barrier Wound Repair Pathway in Drosophila Is Mediated by grainy head. Science 308, 381-385.

MacLellan, W.R., Xiao, G., Abdellatif, M., and Schneider, M.D. (2000). A Novel Rb- and p300Binding Protein Inhibits Transactivation by MyoD. Mol. Cell. Biol. 20, 8903-8915. 
Mai, A., Rotili, D., Tarantino, D., Nebbioso, A., Castellano, S., Sbardella, G., Tini, M., and Altucci, L. (2009). Identification of 4-hydroxyquinolines inhibitors of p300/CBP histone acetyltransferases. Bioorg. Med. Chem. Lett. 19, 1132-1135.

Mai, A., Rotili, D., Tarantino, D., Ornaghi, P., Tosi, F., Vicidomini, C., Sbardella, G., Nebbioso, A., Miceli, M., Altucci, L., and Filetici, P. (2006). Small-Molecule Inhibitors of Histone Acetyltransferase Activity: Identification and Biological Properties. J. Med. Chem. 49, 68976907.

Malouf, G.G., Taube, J.H., Lu, Y., Roysarkar, T., Panjarian, S., Estecio, M.R., Jelinek, J., Yamazaki, J., Raynal, N.J.-M., Long, H., Tahara, T., Tinnirello, A., Ramachandran, P., Zhang, X.-Y., Liang, S., Mani, S.A., and Issa, J.-P.J. (2013). Architecture of epigenetic reprogramming following Twist1-mediated epithelial-mesenchymal transition. Genome Biology 14, 1-17.

Mani, S.A., Guo, W., Liao, M.J., Eaton, E.N., Ayyanan, A., Zhou, A.Y., Brooks, M., Reinhard, F., Zhang, C.C., Shipitsin, M., Campbell, L.L., Polyak, K., Brisken, C., Yang, J., and Weinberg, R.A. (2008). The epithelial-mesenchymal transition generates cells with properties of stem cells. Cell 133, 704-715.

Manning, E.T., Ikehara, T., Ito, T., Kadonaga, J.T., and Kraus, W.L. (2001). p300 Forms a Stable, Template-Committed Complex with Chromatin: Role for the Bromodomain. Mol. Cell. Biol. 21, 3876-3887.

Marcucci, F., Stassi, G., and De Maria, R. (2016). Epithelial-mesenchymal transition: a new target in anticancer drug discovery. Nat Rev Drug Discov 15, 311-325.

Marean, A., Graf, A., Zhang, Y., and Niswander, L. (2011). Folic acid supplementation can adversely affect murine neural tube closure and embryonic survival. Hum. Mol. Gen. 20, 36783683.

Matsuda, S., Harries, J.C., Viskaduraki, M., Troke, P.J.F., Kindle, K.B., Ryan, C., and Heery, D.M. (2004). A Conserved $\alpha$-Helical Motif Mediates the Binding of Diverse Nuclear Proteins to the SRC1 Interaction Domain of CBP. J Biol. Chem. 279, 14055-14064.

Matthews, C.P., Colburn, N.H., and Young, M.R. (2007). AP-1 a target for cancer prevention. Curr Cancer Drug Targets 7, 317-324.

Mehner, C., Hockla, A., Miller, E., Ran, S., Radisky, D.C., and Radisky, E.S. (2014). Tumor cell-produced matrix metalloproteinase 9 (MMP-9) drives malignant progression and metastasis of basal-like triple negative breast cancer. Oncotarget 5, 2736-2749. 
Mehrazarin, S., Chen, W., Oh, J.-E., Liu, Z.X., Kang, K.L., Yi, J.K., Kim, R.H., Shin, K.-H., Park, N.-H., and Kang, M.K. (2015). The p63 Gene Is Regulated by Grainyhead-like 2 (GRHL2) through Reciprocal Feedback and Determines the Epithelial Phenotype in Human Keratinocytes. J Biol. Chem. 290, 19999-20008.

Meidhof, S., Brabletz, S., Lehmann, W., Preca, B.T., Mock, K., Ruh, M., Schüler, J., Berthold, M., Weber, A., Burk, U., Lübbert, M., Puhr, M., Culig, Z., Wellner, U., Keck, T., Bronsert, P., Küsters, S., Hopt, U.T., Stemmler, M.P., and Brabletz, T. (2015). ZEB1-associated drug resistance in cancer cells is reversed by the class I HDAC inhibitor mocetinostat. EMBO Molecular Medicine 7, 831-847.

Merika, M., Williams, A.J., Chen, G., Collins, T., and Thanos, D. (1998). Recruitment of CBP/p300 by the IFNB; Enhanceosome Is Required for Synergistic Activation of Transcription. Mol. Cell 1, 277-287.

Mitra, A., Mishra, L., and Li, S. (2015). EMT, CTCs and CSCs in tumor relapse and drugresistance. Oncotarget 6, 10697-10711.

Miyake, S., Yanagisawa, Y., and Yuasa, Y. (2003). A Novel EID-1 Family Member, EID-2, Associates with Histone Deacetylases and Inhibits Muscle Differentiation. J Biol. Chem. 278, 17060-17065.

Monga, S.P.S., Mars, W.M., Pediaditakis, P., Bell, A., Mulé, K., Bowen, W.C., Wang, X., Zarnegar, R., and Michalopoulos, G.K. (2002). Hepatocyte Growth Factor Induces Wntindependent Nuclear Translocation of $\beta$-Catenin after Met- $\beta$-Catenin Dissociation in Hepatocytes. Cancer Res 62, 2064-2071.

Mujtaba, S., and Zhou, M.-M. (2011). Anti-viral opportunities during transcriptional activation of latent HIV in the host chromatin. Methods 53, 97-101.

Mullighan, C.G., Zhang, J., Kasper, L.H., Lerach, S., Payne-Turner, D., Phillips, L.A., Heatley, S.L., Holmfeldt, L., Collins-Underwood, J.R., Ma, J., Buetow, K.H., Pui, C.-H., Baker, S.D., Brindle, P.K., and Downing, J.R. (2011). CREBBP mutations in relapsed acute lymphoblastic leukaemia. Nature 471, 235-239.

Muraoka, M., Konishi, M., Kikuchi-Yanoshita, R., Tanaka, K., Shitara, N., Chong, J.M., Iwama, T., and Miyaki, M. (1996). p300 gene alterations in colorectal and gastric carcinomas. Oncogene 12, 1565-1569. 
Narayanan, K., Srinivas, R., Peterson, M.C., Ramachandran, A., Hao, J., Thimmapaya, B., Scherer, P.E., and George, A. (2004). Transcriptional Regulation of Dentin Matrix Protein 1 by JunB and p300 during Osteoblast Differentiation. J Biol. Chem. 279, 44294-44302.

Ng, H.H., Robert, F., Young, R.A., and Struhl, K. (2003). Targeted Recruitment of Set1 Histone Methylase by Elongating Pol II Provides a Localized Mark and Memory of Recent Transcriptional Activity. Mol. Cell 11, 709-719.

Nüsslein-Volhard, C., Wieschaus, E., and Kluding, H. Mutations affecting the pattern of the larval cuticle inDrosophila melanogaster. Wilhelm Roux's archives of developmental biology 193, 267-282.

O'Brien, L.E., Zegers, M.M.P., and Mostov, K.E. (2002). Building epithelial architecture: insights from three-dimensional culture models. Nat Rev Mol Cell Biol 3, 531-537.

Ogryzko, V.V., Schiltz, R.L., Russanova, V., Howard, B.H., and Nakatani, Y. (1996). The Transcriptional Coactivators p300 and CBP Are Histone Acetyltransferases. Cell 87, 953-959.

Oike, Y., Takakura, N., Hata, A., Kaname, T., Akizuki, M., Yamaguchi, Y., Yasue, H., Araki, K., Yamamura, K.-i., and Suda, T. (1999). Mice Homozygous for a Truncated Form of CREBBinding Protein Exhibit Defects in Hematopoiesis and Vasculo-angiogenesis. Blood 93, 27712779.

Oliveras-Ferraros, C., Corominas-Faja, B., Cufí, S., Vazquez-Martin, A., Martin-Castillo, B., Iglesias, J.M., López-Bonet, E., Martin, Á.G., and Menendez, J.A. (2012). Epithelial-tomesenchymal transition (EMT) confers primary resistance to trastuzumab (Herceptin). Cell Cycle 11, 4020-4032.

Park, D., Shim, E., Kim, Y., Kim, Y.M., Lee, H., Choe, J., Kang, D., Lee, Y.S., and Jeoung, D. (2008a). C-FLIP promotes the motility of cancer cells by activating FAK and ERK, and increasing MMP-9 expression. Mol Cells 25, 184-195.

Park, S.-M., Gaur, A.B., Lengyel, E., and Peter, M.E. (2008b). The miR-200 family determines the epithelial phenotype of cancer cells by targeting the E-cadherin repressors ZEB1 and ZEB2. Genes Dev. 22, 894-907.

Peinado, H., Ballestar, E., Esteller, M., and Cano, A. (2004). Snail Mediates E-Cadherin Repression by the Recruitment of the Sin3A/Histone Deacetylase 1 (HDAC1)/HDAC2 Complex. Mol. Cell. Biol. 24, 306-319. 
Petrif, F., Giles, R.H., Dauwerse, H.G., Saris, J.J., Hennekam, R.C.M., Masuno, M., Tommerup, N., van Ommen, G.-J.B., Goodman, R.H., Peters, D.J.M., and Breuning, M.H. (1995).

Rubinstein-Taybi syndrome caused by mutations in the transcriptional co-activator CBP. Nature 376, 348-351.

Petrof, G., Nanda, A., Howden, J., Takeichi, T., McMillan, James R., Aristodemou, S., Ozoemena, L., Liu, L., South, Andrew P., Pourreyron, C., Dafou, D., Proudfoot, Laura E., AlAjmi, H., Akiyama, M., McLean, W.H.I., Simpson, Michael A., Parsons, M., and McGrath, John A. (2014). Mutations in GRHL2 Result in an Autosomal-Recessive Ectodermal Dysplasia Syndrome. Am. J. Hum. Genet. 95, 308-314.

Pifer, P.M., Farris, J.C., Thomas, A.L., Stoilov, P., Denvir, J., Smith, D.M., and Frisch, S.M. (2016). Grainyhead-like-2 inhibits the coactivator p300, suppressing tubulogenesis and the epithelial-mesenchymal transition. Mol. Biol. Cell.

Polesskaya, A., Naguibneva, I., Fritsch, L., Duquet, A., Ait-Si-Ali, S., Robin, P., Vervisch, A., Pritchard, L.L., Cole, P., and Harel-Bellan, A. (2001). CBP/p300 and muscle differentiation: no HAT, no muscle. The EMBO Journal 20, 6816-6825.

Pollack, A.L., Apodaca, G., and Mostov, K.E. (2004). Hepatocyte growth factor induces MDCK cell morphogenesis without causing loss of tight junction functional integrity. Am. J. Physiol. 286, C482-C494.

Pollack, A.L., Runyan, R.B., and Mostov, K.E. (1998). Morphogenetic Mechanisms of Epithelial Tubulogenesis: MDCK Cell Polarity Is Transiently Rearranged without Loss of Cell-Cell Contact during Scatter Factor/Hepatocyte Growth Factor-Induced Tubulogenesis. Dev Biol 204, 64-79.

Polyak, K., and Weinberg, R.A. (2009). Transitions between epithelial and mesenchymal states: acquisition of malignant and stem cell traits. Nat Rev Cancer 9, 265-273.

Puri, P.L., Sartorelli, V., Yang, X.-J., Hamamori, Y., Ogryzko, V.V., Howard, B.H., Kedes, L., Wang, J.Y.J., Graessmann, A., Nakatani, Y., and Levrero, M. (1997). Differential Roles of p300 and PCAF Acetyltransferases in Muscle Differentiation. Mol. Cell 1, 35-45.

Pyrgaki, C., Liu, A., and Niswander, L. (2011a). Grainyhead-like 2 regulates neural tube closure and adhesion molecule expression during neural fold fusion. Dev Biol 353, 38-49.

Qin, L., Liu, Z., Chen, H., and Xu, J. (2009). The steroid receptor coactivator-1 regulates twist expression and promotes breast cancer metastasis. Cancer Res 69, 3819-3827. 
Qin, Y., Capaldo, C., Gumbiner, B.M., and Macara, I.G. (2005). The mammalian Scribble polarity protein regulates epithelial cell adhesion and migration through E-cadherin. J Cell Biol 171, 1061-1071.

Quan, Y., Jin, R., Huang, A., Zhao, H., Feng, B., Zang, L., and Zheng, M. (2014).

Downregulation of GRHL2 inhibits the proliferation of colorectal cancer cells by targeting ZEB1. Cancer Biol Ther. 15, 878-887.

Quan, Y., Xu, M., Cui, P., Ye, M., Zhuang, B., and Min, Z. (2015). Grainyhead-like 2 Promotes Tumor Growth and is Associated with Poor Prognosis in Colorectal Cancer. J. Cancer 6, 342350.

Radisky, E.S., and Radisky, D.C. (2010). Matrix Metalloproteinase-Induced EpithelialMesenchymal Transition in Breast Cancer. J Mammary Gland Biol Neoplasia 15, 201-212.

Rifat, Y., Parekh, V., Wilanowski, T., Hislop, N.R., Auden, A., Ting, S.B., Cunningham, J.M., and Jane, S.M. Regional neural tube closure defined by the Grainy head-like transcription factors. Dev Biol 345, 237-245.

Ringel, A.E., and Wolberger, C. (2013). A new RING tossed into an old HAT. Structure 21, 1479-1481.

Roca, H., Hernandez, J., Weidner, S., McEachin, R.C., Fuller, D., Sud, S., Schumann, T., Wilkinson, J.E., Zaslavsky, A., Li, H., Maher, C.A., Daignault-Newton, S., Healy, P.N., and Pienta, K.J. (2013). Transcription Factors OVOL1 and OVOL2 Induce the Mesenchymal to Epithelial Transition in Human Cancer. PLoS ONE 8, e76773.

Roelfsema, J.H., and Peters, D.J.M. (2007). Rubinstein-Taybi syndrome: clinical and molecular overview. Expert Rev Mol Med. 9, 1-16.

Roelfsema, J.H., White, S.J., Ariyürek, Y., Bartholdi, D., Niedrist, D., Papadia, F., Bacino, C.A., den Dunnen, J.T., van Ommen, G.-J.B., Breuning, M.H., Hennekam, R.C., and Peters, D.J.M. (2005). Genetic Heterogeneity in Rubinstein-Taybi Syndrome: Mutations in Both the CBP and EP300 Genes Cause Disease. Am J Hum Genet. 76, 572-580.

Rotte, A., Bhandaru, M., Cheng, Y., Sjoestroem, C., Martinka, M., and Li, G. (2013). Decreased Expression of Nuclear p300 Is Associated with Disease Progression and Worse Prognosis of Melanoma Patients. PLoS ONE 8, e75405. 
Saito, R.-A., Watabe, T., Horiguchi, K., Kohyama, T., Saitoh, M., Nagase, T., and Miyazono, K. (2009). Thyroid Transcription Factor- 1 Inhibits Transforming Growth Factor- $\beta$-Mediated Epithelial-to-Mesenchymal Transition in Lung Adenocarcinoma Cells. Cancer Res 69, 27832791.

Sanchez-Tillo, E., de Barrios, O., Siles, L., Cuatrecasas, M., Castells, A., and Postigo, A. (2012). beta-catenin/TCF4 complex induces the epithelial-to-mesenchymal transition (EMT)-activator ZEB1 to regulate tumor invasiveness. Proc Natl Acad Sci U S A 108, 19204-19209.

Santer, F.R., Hoschele, P.P., Oh, S.J., Erb, H.H., Bouchal, J., Cavarretta, I.T., Parson, W., Meyers, D.J., Cole, P.A., and Culig, Z. (2011a). Inhibition of the acetyltransferases p300 and CBP reveals a targetable function for p300 in the survival and invasion pathways of prostate cancer cell lines. Mol Cancer Ther 10, 1644-1655.

Santos-Rosa, H., Schneider, R., Bannister, A.J., Sherriff, J., Bernstein, B.E., Emre, N.C.T., Schreiber, S.L., Mellor, J., and Kouzarides, T. (2002). Active genes are tri-methylated at K4 of histone H3. Nature 419, 407-411.

Savagner, P., Kusewitt, D.F., Carver, E.A., Magnino, F., Choi, C., Gridley, T., and Hudson, L.G. (2005). Developmental transcription factor slug is required for effective re-epithelialization by adult keratinocytes. J Cell Physiol 202, 858-866.

Scheel, C., Eaton, E.N., Li, S.H., Chaffer, C.L., Reinhardt, F., Kah, K.J., Bell, G., Guo, W., Rubin, J., Richardson, A.L., and Weinberg, R.A. (2011). Paracrine and autocrine signals induce and maintain mesenchymal and stem cell States in the breast. Cell 145, 926-940.

Scheel, C., and Weinberg, R.A. (2011). Phenotypic plasticity and epithelial-mesenchymal transitions in cancer and normal stem cells? Int J Cancer 129, 2310-2314.

Schmidt-Ott, K.M., Yang, J., Chen, X., Wang, H., Paragas, N., Mori, K., Li, J.-Y., Lu, B., Costantini, F., Schiffer, M., Bottinger, E., and Barasch, J. (2005). Novel Regulators of Kidney Development from the Tips of the Ureteric Bud. J. Am. Soc. Nephro.16, 1993-2002.

Schmidt, Johanna M., Panzilius, E., Bartsch, Harald S., Irmler, M., Beckers, J., Kari, V., Linnemann, Jelena R., Dragoi, D., Hirschi, B., Kloos, Uwe J., Sass, S., Theis, F., Kahlert, S., Johnsen, Steven A., Sotlar, K., and Scheel, Christina H. (2015). Stem-Cell-like Properties and Epithelial Plasticity Arise as Stable Traits after Transient Twist1 Activation. Cell Reports 10, 131-139. 
Senga, K., Mostov, K.E., Mitaka, T., Miyajima, A., and Tanimizu, N. (2012). Grainyhead-like 2 regulates epithelial morphogenesis by establishing functional tight junctions through the organization of a molecular network among claudin3, claudin4, and Rab25. Mol. Biol. Cell 23, 2845-2855.

Sharma, S.V., Lee, D.Y., Li, B., Quinlan, M.P., Takahashi, F., Maheswaran, S., McDermott, U., Azizian, N., Zou, L., Fischbach, M.A., Wong, K.-K., Brandstetter, K., Wittner, B., Ramaswamy, S., Classon, M., and Settleman, J. (2010). A chromatin-mediated reversible drug tolerant state in cancer cell subpopulations. Cell 141, 69-80.

Sheppard, H.M., Harries, J.C., Hussain, S., Bevan, C., and Heery, D.M. (2001). Analysis of the Steroid Receptor Coactivator 1 (SRC1)-CREB Binding Protein Interaction Interface and Its Importance for the Function of SRC1. Mol. Biol. Cell 21, 39-50.

Shilatifard, A. (2012). The COMPASS Family of Histone H3K4 Methylases: Mechanisms of Regulation in Development and Disease Pathogenesis. Annu Rev Biochem. 81, 65-95.

Shum, A.S., and Copp, A.J. (1996). Regional differences in morphogenesis of the neuroepithelium suggest multiple mechanisms of spinal neurulation in the mouse. Anatomy And Embryology 194, 65-73.

Singh, B., Shamsnia, A., Raythatha, M.R., Milligan, R.D., Cady, A.M., Madan, S., and Lucci, A. (2014). Highly Adaptable Triple-Negative Breast Cancer Cells as a Functional Model for Testing Anticancer Agents. PLoS ONE 9, e109487.

Siraganian, P.A., Rubinstein, J.H., and Miller, R.W. (1989). Keloids and neoplasms in the Rubinstein-Taybi syndrome. Medical And Pediatric Oncology 17, 485-491.

Skousen, G.J., Wardinsky, T., and Chenaille, P. (1996). Medulloblastoma in patient with Rubinstein-Taybi syndrome. Am. J. Med. Genet 66, 367-367.

Somasundaram, K., Jayaraman, G., Williams, T., Moran, E., Frisch, S., and Thimmapaya, B. (1996). Repression of a matrix metalloprotease gene by E1A correlates with its ability to bind to cell type-specific transcription factor AP-2. Proc. Natl. Acad. Sci. USA 93, 3088-3093.

Stein, R.W., Corrigan, M., Yaciuk, P., Whelan, J., and Moran, E. (1990). Analysis of E1Amediated growth regulation functions: binding of the 300-kilodalton cellular product correlates with E1A enhancer repression function and DNA synthesis-inducing activity. J Virol. 64, 44214427. 
Stiehl, D.P., Fath, D.M., Liang, D., Jiang, Y., and Sang, N. (2007). Histone Deacetylase Inhibitors Synergize p300 Autoacetylation that Regulates Its Transactivation Activity and Complex Formation. Cancer Res 67, 2256-2264.

Stimson, L., Rowlands, M.G., Newbatt, Y.M., Smith, N.F., Raynaud, F.I., Rogers, P., Bavetsias, V., Gorsuch, S., Jarman, M., Bannister, A., Kouzarides, T., McDonald, E., Workman, P., and Aherne, G.W. (2005). Isothiazolones as inhibitors of PCAF and p300 histone acetyltransferase activity. Mol. Cancer Therap. 4, 1521-1532.

Sun, Y., Zeng, X.-R., Wenger, L., Firestein, G.S., and Cheung, H.S. (2004). p53 down-regulates matrix metalloproteinase- 1 by targeting the communications between AP-1 and the basal transcription complex. J. Cell.Biochem. 92, 258-269.

Szabo, R., Molinolo, A., List, K., and Bugge, T.H. (2006). Matriptase inhibition by hepatocyte growth factor activator inhibitor-1 is essential for placental development. Oncogene 26, 15461556.

Taki, T., Sako, M., Tsuchida, M., and Hayashi, Y. (1997). The t(11;16)(q23;p13) Translocation in Myelodysplastic Syndrome Fuses the MLL Gene to the CBP Gene. Blood 89, 3945-3950.

Tam, W.L., and Weinberg, R.A. (2013). The epigenetics of epithelial-mesenchymal plasticity in cancer. Nat Med 19, 1438-1449.

Tanaka, Y., Kanai, F., Tada, M., Tateishi, R., Sanada, M., Nannya, Y., Ohta, M., Asaoka, Y., Seto, M., Shiina, S., Yoshida, H., Kawabe, T., Yokosuka, O., Ogawa, S., and Omata, M. (2008). Gain of GRHL2 is associated with early recurrence of hepatocellular carcinoma. J. Hepat.49, 746-757.

Tang, Z., Chen, W.-Y., Shimada, M., Nguyen, U.T.T., Kim, J., Sun, X.-J., Sengoku, T., McGinty, R.K., Fernandez, J.P., Muir, T.W., and Roeder, R.G. (2013). SET1 and p300 Act Synergistically, through Coupled Histone Modifications, in Transcriptional Activation by p53. Cell 154, 297-310.

Tanimizu, N., Kobayashi, S., Ichinohe, N., and Mitaka, T. (2014). Downregulation of miR122 by grainyhead-like 2 restricts the hepatocytic differentiation potential of adult liver progenitor cells. Development 141, 4448-4456.

Tanimizu, N., Nakamura, Y., Ichinohe, N., Mizuguchi, T., Hirata, K., and Mitaka, T. (2013). Hepatic biliary epithelial cells acquire epithelial integrity but lose plasticity to differentiate into hepatocytes in vitro during development. J. Cell Sci.126, 5239-5246. 
Taube, J.H., Herschkowitz, J.I., Komurov, K., Zhou, A.Y., Gupta, S., Yang, J., Hartwell, K., Onder, T.T., Gupta, P.B., Evans, K.W., Hollier, B.G., Ram, P.T., Lander, E.S., Rosen, J.M., Weinberg, R.A., and Mani, S.A. (2010). Core epithelial-to-mesenchymal transition interactome gene-expression signature is associated with claudin-low and metaplastic breast cancer subtypes. Proc Natl Acad Sci U S A 107, 15449-15454.

Teo, J.-L., and Kahn, M. (2010). The Wnt signaling pathway in cellular proliferation and differentiation: A tale of two coactivators. Advanced Drug Delivery Reviews 62, 1149-1155.

Terpstra, W., Prins, A., Ploemacher, R.E., Wognum, B.W., Wagemaker, G., Lowenberg, B., and Wielenga, J.J. (1996). Long-term leukemia-initiating capacity of a CD34-subpopulation of acute myeloid leukemia. Blood 87, 2187-2194.

Thakur, J.K., Yadav, A., and Yadav, G. (2013). Molecular recognition by the KIX domain and its role in gene regulation. Nucleic Acids Res.

Thiery, J.P., Acloque, H., Huang, R.Y., and Nieto, M.A. (2009). Epithelial-mesenchymal transitions in development and disease. Cell 139, 871-890.

Thiery, J.P., and Sleeman, J.P. (2006). Complex networks orchestrate epithelial-mesenchymal transitions. Nat Rev Mol Cell Biol 7, 131-142.

Tian, X., Liu, Z., Niu, B., Zhang, J., Tan, T.K., Lee, S.R., Zhao, Y., Harris, D.C.H., and Zheng, G. (2011). E-Cadherin/ $\beta$-Catenin Complex and the Epithelial Barrier. J Biomed Biotechnol. 2011, 567305.

Tillinghast, G.W., Partee, J., Albert, P., Kelley, J.M., Burtow, K.H., and Kelly, K. (2003). Analysis of genetic stability at the EP300 and CREBBP loci in a panel of cancer cell lines. Genes Chromosomes Cancer 37, 121-131.

Ting, S.B., Wilanowski, T., Auden, A., Hall, M., Voss, A.K., Thomas, T., Parekh, V., Cunningham, J.M., and Jane, S.M. (2003a). Inositol- and folate-resistant neural tube defects in mice lacking the epithelial-specific factor Grhl-3. Nat Med 9, 1513-1519.

Ting, S.B., Wilanowski, T., Cerruti, L., Zhao, L.L., Cunningham, J.M., and Jane, S.M. (2003b). The identification and characterization of human Sister-of-Mammalian Grainyhead (SOM) expands the grainyhead-like family of developmental transcription factors. Biochem J 370, 953962. 
Traylor-Knowles, N., Hansen, U., Dubuc, T.Q., Martindale, M.Q., Kaufman, L., and Finnerty, J.R. (2010). The evolutionary diversification of LSF and Grainyhead transcription factors preceded the radiation of basal animal lineages. BMC Evolutionary Biology 10, 1-13.

Truong, A.B., Kretz, M., Ridky, T.W., Kimmel, R., and Khavari, P.A. (2006). p63 regulates proliferation and differentiation of developmentally mature keratinocytes. Genes Dev 20, 31853197.

Turksen, K., and Troy, T.-C. (2011). Junctions gone bad: Claudins and loss of the barrier in cancer. Biochim. Biophys. Acta Reviews on Cancer 1816, 73-79.

Varier, R.A., Swaminathan, V., Balasubramanyam, K., and Kundu, T.K. (2004). Implications of small molecule activators and inhibitors of histone acetyltransferases in chromatin therapy. Biochem. Pharm. 68, 1215-1220.

Varma, S., Cao, Y., Tagne, J.-B., Lakshminarayanan, M., Li, J., Friedman, T.B., Morell, R.J., Warburton, D., Kotton, D.N., and Ramirez, M.I. (2012). The Transcription Factors Grainyheadlike 2 and NK2-Homeobox 1 Form a Regulatory Loop That Coordinates Lung Epithelial Cell Morphogenesis and Differentiation. J. Biol. Chem.287, 37282-37295.

Varma, S., Mahavadi, P., Sasikumar, S., Cushing, L., Hyland, T., Rosser, A.E., Riccardi, D., Lu, J., Kalin, T.V., Kalinichenko, V.V., Guenther, A., Ramirez, M.I., Pardo, A., Selman, M., and Warburton, D. (2014). Grainyhead-like 2 (GRHL2) distribution reveals novel pathophysiological differences between human idiopathic pulmonary fibrosis and mouse models of pulmonary fibrosis. Am. J. of Physiol. - Lung Cellular and Molecular Physiology 306, L405-L419.

Vestweber, D., and Kemler, R. (1985). Identification of a putative cell adhesion domain of uvomorulin. The EMBO Journal 4, 3393-3398.

Viosca, J., Lopez-Atalaya, J.P., Olivares, R., Eckner, R., and Barco, A. (2010). Syndromic features and mild cognitive impairment in mice with genetic reduction on p300 activity: Differential contribution of p300 and CBP to Rubinstein-Taybi syndrome etiology. Neurobiology of Disease 37, 186-194.

Vleugel, M.M., Shvarts, D., van der Wall, E., and van Diest, P.J. (2006). p300 and p53 levels determine activation of HIF-1 downstream targets in invasive breast cancer. Hum Pathol 37.

Vo, N., and Goodman, R.H. (2001). CREB-binding Protein and p300 in Transcriptional Regulation. J. Biol. Chem. 276, 13505-13508. 
von Burstin, J., Eser, S., Paul, M.C., Seidler, B., Brandl, M., Messer, M., von Werder, A., Schmidt, A., Mages, J., Pagel, P., Schnieke, A., Schmid, R.M., Schneider, G., and Saur, D. (2009). E-Cadherin Regulates Metastasis of Pancreatic Cancer In Vivo and Is Suppressed by a SNAIL/HDAC1/HDAC2 Repressor Complex. Gastroenterology 137, 361-371.e365.

Walentin, K., Hinze, C., Werth, M., Haase, N., Varma, S., Morell, R., Aue, A., Pötschke, E., Warburton, D., Qiu, A., Barasch, J., Purfürst, B., Dieterich, C., Popova, E., Bader, M., Dechend, R., Staff, A.C., Yurtdas, Z.Y., Kilic, E., and Schmidt-Ott, K.M. (2015). A Grhl2-dependent gene network controls trophoblast branching morphogenesis. Development 142, 1125-1136.

Wang, A.Z., Ojakian, G.K., and Nelson, W.J. (1990). Steps in the morphogenesis of a polarized epithelium. I. Uncoupling the roles of cell-cell and cell-substratum contact in establishing plasma membrane polarity in multicellular epithelial (MDCK) cysts. J. Cell Sci. 95, 137-151.

Wang, F., Marshall, C.B., and Ikura, M. (2013). Transcriptional/epigenetic regulator CBP/p300 in tumorigenesis: structural and functional versatility in target recognition. Cell. Mol. Life Sci, 70, 3989-4008.

Warzecha, C.C., Jiang, P., Amirikian, K., Dittmar, K.A., Lu, H., Shen, S., Guo, W., Xing, Y., and Carstens, R.P. (2010). An ESRP-regulated splicing programme is abrogated during the epithelial-mesenchymal transition. The EMBO Journal 29, 3286-3300.

Warzecha, C.C., Sato, T.K., Nabet, B., Hogenesch, J.B., and Carstens, R.P. (2009). ESRP1 and ESRP2 Are Epithelial Cell-Type-Specific Regulators of FGFR2 Splicing. Mol. Cell 33, 591-601.

Watanabe, K., Villarreal-Ponce, A., Sun, P., Salmans, Michael L., Fallahi, M., Andersen, B., and Dai, X. (2014). Mammary Morphogenesis and Regeneration Require the Inhibition of EMT at Terminal End Buds by Ovol2 Transcriptional Repressor. Dev. Cell 29, 59-74.

Watson, J.D.a.C., F. H. C. . (1953). Molecular Structure of Nucleic acids Nature 4356.

Wellner, U., Schubert, J., Burk, U.C., Schmalhofer, O., Zhu, F., Sonntag, A., Waldvogel, B., Vannier, C., Darling, D., Hausen, A.z., Brunton, V.G., Morton, J., Sansom, O., Schuler, J., Stemmler, M.P., Herzberger, C., Hopt, U., Keck, T., Brabletz, S., and Brabletz, T. (2009). The EMT-activator ZEB1 promotes tumorigenicity by repressing stemness-inhibiting microRNAs. Nat Cell Biol 11, 1487-1495.

Werner, S., Frey, S., Riethdorf, S., Schulze, C., Alawi, M., Kling, L., Vafaizadeh, V., Sauter, G., Terracciano, L., Schumacher, U., Pantel, K., and Assmann, V. (2013). Dual roles of the transcription factor grainyhead-like 2 (GRHL2) in breast cancer. J Biol Chem 288, 22993-23008. 
Werth, M., Walentin, K., Aue, A., Schönheit, J., Wuebken, A., Pode-Shakked, N., Vilianovitch, L., Erdmann, B., Dekel, B., Bader, M., Barasch, J., Rosenbauer, F., Luft, F.C., and Schmidt-Ott, K.M. (2010). The transcription factor grainyhead-like 2 regulates the molecular composition of the epithelial apical junctional complex. Development 137, 3835-3845.

Westermarck, J., and KÄHÄRi, V.-M. (1999). Regulation of matrix metalloproteinase expression in tumor invasion. The FASEB Journal 13, 781-792.

Wilanowski, T., Tuckfield, A., Cerruti, L., O'Connell, S., Saint, R., Parekh, V., Tao, J., Cunningham, J.M., and Jane, S.M. (2002). A highly conserved novel family of mammalian developmental transcription factors related to Drosophila grainyhead. Mech Dev 114, 37-50.

Witte, S., Bradley, A., Enright, A.J., and Muljo, S.A. (2015). High-density P300 enhancers control cell state transitions. BMC Genomics 16, 1-13.

Woodward, W.A., Chen, M.S., Behbod, F., Alfaro, M.P., Buchholz, T.A., and Rosen, J.M. (2007). WNT/beta-catenin mediates radiation resistance of mouse mammary progenitor cells. Proc Natl Acad Sci U S A 104, 618-623.

Wu, M., Wang, P.F., Lee, J.S., Martin-Brown, S., Florens, L., Washburn, M., and Shilatifard, A. (2008). Molecular Regulation of H3K4 Trimethylation by Wdr82, a Component of Human Set1/COMPASS. Mol. Cell. Biol. 28, 7337-7344.

Xiang, J., Fu, X., Ran, W., Chen, X., Hang, Z., Mao, H., and Wang, Z. (2013). Expression and role of grainyhead-like 2 in gastric cancer. Medical Oncology 30, 1-7.

Xiang, X., Deng, Z., Zhuang, X., Ju, S., Mu, J., Jiang, H., Zhang, L., Yan, J., Miller, D., and Zhang, H.-G. (2012). Grhl2 Determines the Epithelial Phenotype of Breast Cancers and Promotes Tumor Progression. PLoS ONE 7, e50781.

Xu, J., Lamouille, S., and Derynck, R. (2009). TGF-[beta]-induced epithelial to mesenchymal transition. Cell Res 19, 156-172.

Xu, Y., Hu, B., Qin, L., Zhao, L., Wang, Q., Wang, Q., Xu, Y., and Jiang, J. (2014). SRC-1 and Twist1 expression positively correlates with a poor prognosis in human breast cancer. Int J Biol Sci 10, 396-403.

Yang, H., Pinello, C.E., Luo, J., Li, D., Wang, Y., Zhao, L.Y., Jahn, S.C., Saldanha, S.A., Chase, P., Planck, J., Geary, K.R., Ma, H., Law, B.K., Roush, W.R., Hodder, P., and Liao, D. (2013a). 
Small-molecule inhibitors of acetyltransferase p300 identified by high-throughput screening are potent anticancer agents. Mol Cancer Ther 12, 610-620.

Yang, X., Vasudevan, P., Parekh, V., Penev, A., and Cunningham, J.M. (2013b). Bridging Cancer Biology with the Clinic: Relative Expression of a GRHL2-Mediated Gene-Set Pair Predicts Breast Cancer Metastasis. PLoS ONE 8, e56195.

Yao, T.-P., Oh, S.P., Fuchs, M., Zhou, N.-D., Ch'ng, L.-E., Newsome, D., Bronson, R.T., Li, E., Livingston, D.M., and Eckner, R. Gene Dosage\&\#x2013;Dependent Embryonic Development and Proliferation Defects in Mice Lacking the Transcriptional Integrator p300. Cell 93, 361-372.

Ye, X., and Weinberg, R.A. (2015). Epithelial-Mesenchymal Plasticity: A Central Regulator of Cancer Progression. Trends in Cell Biology 25, 675-686.

Yeaman, C., Grindstaff, K.K., Hansen, M.D.H., and Nelson, W.J. (1999). Cell polarity: Versatile scaffolds keep things in place. Current Biology 9, R515-R517.

Yie, J., Senger, K., and Thanos, D. (1999). Mechanism by which the IFN- $\beta$ enhanceosome activates transcription. Proc. Natl. Acad. Sci. USA 96, 13108-13113.

Yokoyama, A., Wang, Z., Wysocka, J., Sanyal, M., Aufiero, D.J., Kitabayashi, I., Herr, W., and Cleary, M.L. (2004). Leukemia Proto-Oncoprotein MLL Forms a SET1-Like Histone Methyltransferase Complex with Menin To Regulate Hox Gene Expression. Mol. Cell. Biol. 24, 5639-5649.

Younis, L.K., El Sakka, H., and Haque, I. (2007). The Prognostic Value of E-cadherin Expression in Breast Cancer. International Journal of Health Sciences 1, 43-51.

Yu, M., Bardia, A., Wittner, B.S., Stott, S.L., Smas, M.E., Ting, D.T., Isakoff, S.J., Ciciliano, J.C., Wells, M.N., Shah, A.M., Concannon, K.F., Donaldson, M.C., Sequist, L.V., Brachtel, E., Sgroi, D., Baselga, J., Ramaswamy, S., Toner, M., Haber, D.A., and Maheswaran, S. (2013). Circulating Breast Tumor Cells Exhibit Dynamic Changes in Epithelial and Mesenchymal Composition. Science 339, 580-584.

Yuan, J., Dutton, C.M., and Scully, S.P. (2005). RNAi mediated MMP-1 silencing inhibits human chondrosarcoma invasion. J Orthop Res. 23, 1467-1474. 
Zeisberg, M., Bottiglio, C., Kumar, N., Maeshima, Y., Strutz, F., Müller, G.A., and Kalluri, R. (2003). Bone morphogenic protein-7 inhibits progression of chronic renal fibrosis associated with two genetic mouse models. Am J Physiol Renal Physiol 285, F1060-F1067.

Zhang, L., He, X., Liu, L., Jiang, M., Zhao, C., Wang, H., He, D., Zheng, T., Zhou, X., Hassan, A., Ma, Z., Xin, M., Sun, Z., Lazar, Mitchell A., Goldman, Steven A., Olson, Eric N., and Lu, Q.R. (2016). Hdac3 Interaction with p300 Histone Acetyltransferase Regulates the Oligodendrocyte and Astrocyte Lineage Fate Switch. Dev. Cell 36, 316-330.

Zhang, P., Sun, Y., and Ma, L. (2015). ZEB1: At the crossroads of epithelial-mesenchymal transition, metastasis and therapy resistance. Cell Cycle 14, 481-487.

Zhang, Y., Yan, W., and Chen, X. (2014). P63 regulates tubular formation via epithelial-tomesenchymal transition. Oncogene 33, 1548-1557.

Zhao, T., Satou, Y., Sugata, K., Miyazato, P., Green, P.L., Imamura, T., and Matsuoka, M. (2011). HTLV-1 bZIP factor enhances TGF- $\beta$ signaling through p300 coactivator. Blood 118, 1865-1876.

Zhou, B., Liu, Y., Kahn, M., Ann, D.K., Han, A., Wang, H., Nguyen, C., Flodby, P., Zhong, Q., Krishnaveni, M.S., Liebler, J.M., Minoo, P., Crandall, E.D., and Borok, Z. (2012). Interactions between beta-catenin and transforming growth factor-beta signaling pathways mediate epithelialmesenchymal transition and are dependent on the transcriptional co-activator cAMP-response element-binding protein (CREB)-binding protein (CBP). J Biol Chem 287, 7026-7038.

Zhou, X.Y., Shibusawa, N., Naik, K., Porras, D., Temple, K., Ou, H., Kaihara, K., Roe, M.W., Brady, M.J., and Wondisford, F.E. (2004). Insulin regulation of hepatic gluconeogenesis through phosphorylation of CREB-binding protein. Nature Medicine 10, 633-637. 
Figure 1. p300 protein. A. Domains of p300 B. Ribbon and surface representations of p300 core structures.

a
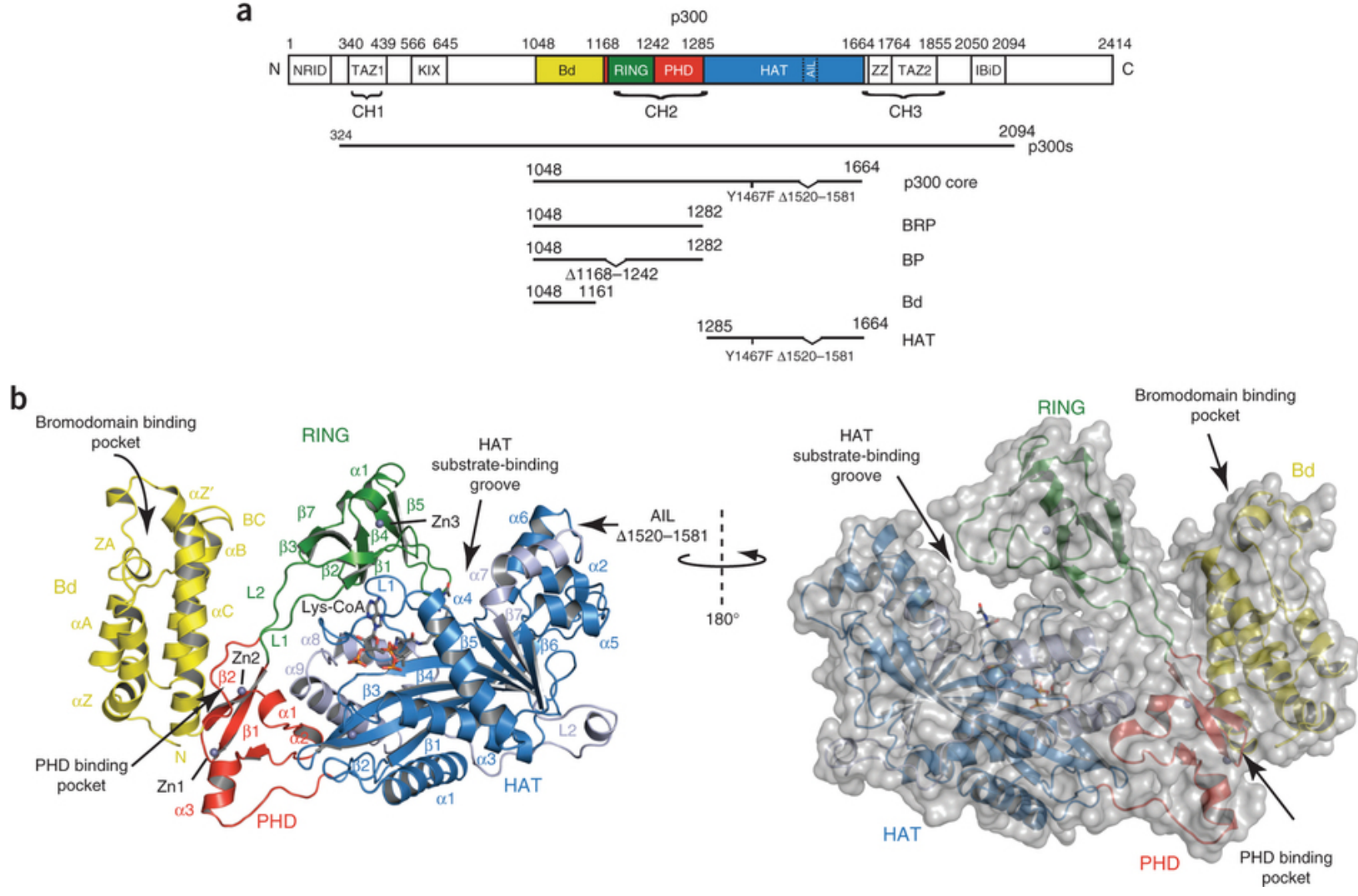

Figure 1

Reprinted by permission from Macmillan Publishers Ltd: [Nature Structural and Molecular Biology], Structure of the p300 catalytic core and implications for chromatin targeting and HAT regulation, 3856130394085, 2013 


\section{Chapter 2}

Grainyhead-like-2 inhibits the co-activator p300, suppressing tubulogenesis and the epithelial-mesenchymal transition

Published in: Molecular Biology of the Cell

Mol. Biol. Cell. 2016 Jun 1. doi: 10.1091/mbc.E16-04-0249 
Grainyhead-like-2 inhibits the co-activator p300, suppressing tubulogenesis and the epithelial-mesenchymal transition

Phillip M. Pifer ${ }^{1}$, Joshua C. Farris ${ }^{1}$, Alyssa L.Thomas ${ }^{1}$, Peter Stoilov² James Denvir ${ }^{3}$, David M. Smith $^{2}$ and Steven M. Frisch ${ }^{1,2^{*}}$

1-Mary Babb Randolph Cancer Center

1 Medical Center Drive, Campus Box 9300

West Virginia University

Morgantown, WV 26506

2 -Department of Biochemistry

1 Medical Center Drive

West Virginia University

Morgantown, WV 26506

3-Department of Biochemistry and Microbiology

Marshall University

One John Marshall Drive

Huntington, West Virginia 25755

*Address for correspondence: Steven M. Frisch, Mary Babb Randolph Cancer Center, 1 Medical Center Drive, Room 2833, West Virginia University, Morgantown, WV 26506.

Email: $\underline{\text { frisch@hsc.wvu.edu }}$ 


\begin{abstract}
Developmental morphogenesis and tumor progression require a transient or stable breakdown of epithelial junctional complexes to permit programmed migration, invasion and anoikis-resistance, characteristics endowed by the Epithelial-Mesenchymal Transition. The epithelial master-regulatory transcription factor Grainyhead-like 2 (GRHL2) suppresses and reverses EMT, causing a mesenchymal-epithelial transition to the default epithelial phenotype. Here, we investigated the role of GRHL2 in tubulogenesis of Madin-Darby Canine Kidney (MDCK) cells, a process requiring transient, partial EMT. GRHL2 was required for cystogenesis, but it suppressed tubulogenesis in response to Hepatocyte Growth Factor (HGF). Surprisingly, GRHL2 suppressed this process by inhibiting the histone acetyltransferase coactivator, p300, preventing the induction of matrix metalloproteases and other p300-dependent genes required for tubulogenesis. A thirteen amino acid region of GRHL2 was necessary and sufficient for the inhibition of p300, the suppression of tubulogenesis and the interference with EMT. The results demonstrate that p300 is required for the partial or complete EMT occurring in tubulogenesis or tumor progression, and that GRHL2 suppresses EMT in both contexts, through inhibition of p300.
\end{abstract}




\section{INTRODUCTION}

p300 is a co-activator of transcription that interacts with at least four hundred DNAfactors to activate transcription from thousands of enhancers/promoters, mostly contingent upon its intrinsic histone acetyltransferase (HAT) activity. In this context, it is viewed as an “integrator" factor that serves as a nexus among multiple signaling pathways and programs of gene expression (Yao et al.; Kamei et al., 1996; Vo and Goodman, 2001; Bedford et al., 2010). Accordingly, p300/CBP null mice die at E9.5-11.5 with massive deficits of mesenchymal cells and erythroid cells, as well as defects in neurulation and heart development (Yao et al.; Oike et al., 1999). p300 is essential for the differentiation of muscle, erythroid lineages, osteoblast, oligodendrocytes, and stem cells (Puri et al., 1997; Oike et al., 1999; Blobel, 2000; Polesskaya et al., 2001; Narayanan et al., 2004; Teo and Kahn, 2010; Zhang et al., 2016). A recent study has demonstrated p300 is a marker for super-enhancers, positioning it to control cell state transitions (Witte et al., 2015).

Grainyhead-like 2 (GRHL2) is one of the three genes comprising a family of mammalian transcription factors related to Drosophila Grainyhead, the first zygotically encoded transcription factor expressed during the maternal to zygotic transition (the MZT) (Harrison et al.). GRHL2 is important for epithelial barrier assembly in, for example, the morphogenesis of kidney collecting ducts, placenta, lung alveoli and the mammary gland ductal system (through OVOL2, a GRHL2 target gene) (Gao et al., 2013; Watanabe et al., 2014; Aue et al., 2015; Walentin et al., 2015). Grainyhead factors are also critical for diverse developmental events that involve the formation of epithelial barriers, including epidermal assembly, neural crest formation, and, in the adult, wound-healing (Wilanowski et al., 2002; Gustavsson et al., 2008; Wang and Samakovlis, 2012; Mlacki et al., 2015). GRHL2 activates the expression of multiple genes encoding epithelial cell 
adhesion molecules (e.g., E-cadherin, desmosomal proteins, tight junction proteins), and due to its widespread expression, may be generally important for enforcing an epithelial phenotype (Rifat et al.; Senga et al.; Wilanowski et al., 2002; Ting et al., 2003; Ting et al., 2005; Auden et al., 2006; Werth et al., 2010; Boglev et al., 2011; Cieply et al., 2012; Tanimizu and Mitaka, 2013; Aue et al., 2015). Correspondingly, GRHL2 expression plays an important role in suppressing the oncogenic EMT, thereby functioning as a tumor suppressor that enhances anoikis-sensitivity (Cieply et al., 2012; Cieply et al., 2013; Mlacki et al., 2015); in fact, the gene most tightly correlated with E-cadherin expression in human tumors was GRHL2 (Kohn et al., 2014).

The opposing roles of GRHL2 in establishing the epithelial state vs. p300 in differentiation - including EMT -- motivated us to investigate a potential role for GRHL2 in inhibiting p300 function. Kidney tubulogenesis is contingent upon temporal and spatial regulation of epithelial cells, in which GRHL2, which is expressed in the ureteric buds of the developing kidney plays a critical organizing role (Schmidt-Ott et al., 2005; Aue et al., 2015; Walentin et al., 2015). Madin-Darby Canine Kidney (MDCK) tubulogenesis in response to hepatocyte growth factor (HGF) models some aspects of kidney collecting duct tubulogenesis in vivo and requires a transient, partial EMT (pEMT) (Pollack et al., 1998; O'Brien et al., 2002; Pollack et al., 2004; Leroy and Mostov, 2007; Hellman et al., 2008; Jung et al., 2012; Zhang et al., 2014). HGF causes MDCK cells to undergo cell scattering in two-dimensional culture, and induces tubulogenesis of MDCK cysts that form in a three-dimensional collagen gel (Wang et al., 1990,1991 \#4686; Pollack et al., 2004). Induction of MMP1 and MMP13 by HGF through AP1/p300 complexes is required for tubulogenesis in the MDCK model (Benbow and Brinckerhoff, 1997; Clark et al., 2008; Hellman et al., 2008; Chacon-Heszele et al., 2014). 
Targeted knockout studies indicate a role for HGF/Met in kidney development, and, relatedly, HGF/Met signaling is a potent stimulator of Wnt signaling, a crucial signaling pathway in this

process, validating the in vivo relevance of this approach (Monga et al., 2002; Bridgewater et al., 2008; Ishibe et al., 2009).

Herein, we report that GRHL2 promoted cystogenesis but suppressed tubulogenesis. GRHL2 protein interacted functionally with p300, inhibiting its HAT activity and transcriptional activation of target genes, including matrix metalloproteases. A small (thirteen amino acid) sequence of GRHL2 was important for inhibition of p300, suppression of tubulogenesis and the reversal of EMT. These results mechanistically position GRHL2, an enforcer of the epithelial default phenotype, as an antagonist of p300, a co-activator of differentiation-specific genes, with important ramifications for developmental and tumor biology.

\section{RESULTS}

GRHL2 suppresses cell scattering and tubulogenesis

In light of the epithelial programming role of GRHL2, we tested the effect of HGF on GRHL2 levels in MDCK cells. Interestingly, we found that HGF down-regulated the expression of endogenous GRHL2 protein levels at early time points (figure 1A). When constitutively expressed in MDCK cells, GRHL2 significantly inhibited HGF-induced cell scattering compared to vector control cells (figure 1B). Conversely, MDCK cells with GRHL2 shRNA knockdown demonstrated enhanced HGF-induced cell scattering; retention of E-cadherin expression showed that EMT did not occur in response to the knockdown of GRHL2 alone (figure 1C and see video 
in supplemental figure S1). GRHL2 had only modest effects on the phosphorylation status of two established HGF/Met signaling mediators, Erk and Akt (figure S2).

We then examined the effects of GRHL2 on HGF-induced tubulogenesis in the threedimensional collagen model. GRHL2 expression caused the formation of larger cysts compared to vector control cells, consistent with previous reports in hepatic bile duct cells (figure 1D) (Tanimizu and Mitaka, 2013). GRHL2 significantly suppressed tubulogenesis/branching morphogenesis following HGF treatment (figure 1D); a similar effect of murine Grhl2 was observed in mouse inner medullary collecting duct cell line (figure S3A), indicating the important role of GRHL2 down-regulation in tubulogenesis. Conversely, the stable knockdown of GRHL2 prevented cystogenesis (figure S3B), presumably by down-regulating epithelial adhesion molecules (Senga et al.; Werth et al., 2010). Transient knockdown of GRHL2 following cyst formation using a doxycycline-induced shRNA vector, however, clearly indicated that the loss of GRHL2 promoted tubulogenesis (figure S4A). There was no effect of the GRHL2 shRNA on cell proliferation (figure S4B). These results indicated that GRHL2 suppressed HGF-activated cell scattering and tubulogenesis.

\section{GRHL2 suppresses the expression of matrix metalloproteases}

To identify GRHL2 target genes responsible for the attenuation of cellular responses to HGF/Met signaling, RNA-Seq was used to compare MDCK cells with constitutive GRHL2 expression vs. GRHL2 depletion, either with no treatment or with HGF induction (24 hours). This experimental scheme allowed for two distinct comparisons: genes regulated by HGF in the absence vs. presence of GRHL2, and genes regulated by GRHL2 with vs. without HGF induction. GRHL2 down-regulated several known matrix metalloproteases (MMP) family members (Table 1, confirmed by qPCR in figure 2A). In light of the established importance of 
specific MMPs for tubulogenesis in the MDCK and mIMCD-3 cell culture models (Hotary et al., 2000; Jorda et al., 2005; Hellman et al., 2008; Chacon-Heszele et al., 2014), we verified their importance for branching tubulogenesis in embryonic kidneys cultured ex-vivo using the generalized MMP inhibitor, batimastat (figure S5). The down-regulation of MMPs by GRHL2 could contribute significantly to the tubulogenesis-suppressing effect of constitutive GRHL2 expression, although additional contributions from EMT/MET-related GRHL2 genes are likely.

To investigate the transcriptional mechanisms of MMP gene regulation by GRHL2, MMP1 and MMP14 promoters were assayed as luciferase reporter constructs by cotransfection in HT1080 fibrosarcoma cells, in which the endogenous MMPs are expressed constitutively (Tang and Hemler, 2004; Nonaka et al., 2005). GRHL2 was found to suppress MMP1 and MMP14 promoters using adenovirus E1a protein, a known repressor of $M M P$ genes, as a positive control (figure 2B). These results demonstrated that GRHL2 repressed MMP1 and MMP14 promoters; however, examination of the promoter sequences utilized in our reporters constructs did not indicate the presence of GRHL2 DNA binding consensus sites (Wilanowski et al., 2002; Gao et al., 2013; Aue et al., 2015; Walentin et al., 2015), suggesting a more subtle mechanism for repression not involving direct DNA binding.

\section{GRHL2 inhibits p300 function}

The AP-1 transcription factor family is important in the regulation of most MMP genes (Clark et al., 2008). GRHL2 significantly suppressed AP-1 activity on minimal reporter constructs in HT1080 and 293 cells (figure 3A). GRHL2 did not appear to affect the expression of FOS or JUN family members following HGF induction (figure S6). 
In light of the functional similarities between adenovirus E1a and GRHL2 (see Discussion), we compared a published list of genes that E1a down-regulated through p300 interaction (Ferrari et al., 2014) against our list of GRHL2-downregulated genes; this latter list was derived from the RNA-seq analysis that we performed on GRHL2-expressing human mesenchymal subpopulation cells (MSP), reported in (Farris et al., 2016) (as comparison of canine vs. human gene lists proved uninformative for technical reasons). About $43.5 \%$ of the genes that E1a repressed through p300 were also repressed by GRHL2 (figure 3B). Downstream Effector Analysis/Ingenuity Pathway analysis revealed that the expression of GRHL2 suppressed the regulation of numerous p300-associated genes that were up-regulated or down-regulated by HGF in the absence of GRHL2 expression (figure 3C and figure S7). These results suggested that GRHL2 could potentially inhibit p300 function.

To investigate this, the effect of GRHL2 upon the activity of a GAL4-DNA binding domain-p300 fusion protein was assayed by transient transfection using a GAL4-responsive luciferase promoter, an established method for testing p300 coactivator function without confounding effects from primary DNA binding activator proteins (Snowden et al., 2000). Adenovirus E1a protein repressed the AP-1 and GAL4/GAL4-p300 reporter activities, but a p300-non-binding mutant of E1a (Ferrari et al., 2014) failed to do so (figure S8), validating the p300-dependence of transcription in these assays. GRHL2 inhibited the co-activator function of p300 in this assay (figure 3D). As additional negative controls, GRHL2 did not affect the ability of a p300-independent GAL4-activator fusion protein (VP16-GAL4; (Kutluay et al., 2009)) to activate the same reporter; another transcription factor unrelated to GRHL2, c-Myc, failed to inhibit GAL4-p300 function in this assay (figure S9). These results indicated that GRHL2 was a 
potent suppressor of p300 function. The highly related GRHL1 and GRHL3 proteins were found to have similar effects in the reporter assay (figure S10).

GRHL2 inhibits the histone acetyltransferase (HAT) activity of p300

The HAT domain of p300 acetylates numerous substrates, including lysines 18 and 27 in the N-terminal tail of histone 3 (H3) (Ogryzko et al., 1996; Kasper et al., 2010; Jin et al., 2011). Using recombinant proteins, GRHL2 inhibited acetylation of H3K27 by p300 in a dosedependent manner in vitro (figure 4A). Similar results were obtained using the p300 HAT domain alone (amino acids1283-1673) rather than full-length p300 (figure S11). Recombinant GRHL1 and GRHL3 proteins also inhibited the HAT activity of p300 (figure S12).

To confirm these in vitro effects in cells, we analyzed histone acetylation by CHIP-qPCR using the human cell line, HT1080 (as Encode reference data reporting histone acetylation are available for the human genome.) Consistent with the in vitro results, the acetylation of H3K27 was inhibited in HT1080 cells expressing GRHL2 constitutively, specifically on promoters that GRHL2 repressed, including MMP1, MMP14, MMP2, and ZEB1; acetylation of GRHL2upregulated gene promoter-associated H3K27 was either not affected (CDH1, ESRP1) or upregulated (RAB25) (figure 4B). Unlike the effect of E1a on p300, there was neither an effect of GRHL2 on global H3 acetylation nor global chromatin condensation, assayed by Western blotting (figure 4B) or by immunofluorescent localization of a LacI-mCherry-GRHL2 protein (Ferrari et al., 2014), respectively (figure S13).

GRHL2 interacted with p300 by the criteria of co-immunoprecipitation of transiently cotransfected genes, or of endogenous proteins (figure 4C). The recombinant proteins interacted 
only weakly, however (figure S14) suggesting that the interaction is transient or requires posttranslational modifications absent from our recombinant proteins.

GRHL2 inhibits the C-terminal transactivation domain of P300

Multiple domains of p300 can co-activate transcription independently. One of these is the C-terminal domain (1665aa-2414aa) which interacts with the p160 family of co-activators (Stiehl et al., 2007). Previous reports suggested that the activity of the C-terminal domain is stimulated by auto-acetylation, catalyzed by the p300 HAT domain (Stiehl et al., 2007). In light of the HAT-inhibitory effect of GRHL2 described above, we hypothesized that GRHL2 inhibited the C-terminal domain through this mechanism. We confirmed that transcriptional activation by the C-terminal domain, assayed as a GAL4-C-term fusion protein, was stimulated by a cotransfected p300 HAT domain, and that the latter domain had no detectable ability to activate transcription independently (figure 5A). GRHL2 inhibited the enhanced transactivation by the p300 C-terminal domain in the presence of co-transfected p300 HAT (figure 5B). Embedded within the C-terminal domain is a small sub-domain called the IRF-3 Binding Domain (IBID; amino acids 2050-2096) that mediates the interaction with the p160 co-activator family, including the family member Steroid Receptor Coactivator-1 (SRC-1) (Sheppard et al., 2001). While neither the IBID domain of p300 nor SRC-1 alone activated transcription efficiently, the combination of both activated transcription, as reported previously (figure 5C) (Sheppard et al., 2001). GRHL2 potently inhibited transcription that was driven by the complex of p300-IBID and SRC-1 (figure 5D). Interestingly, mutation of the two lysines (K2086 and K2091) of the IBID domain that are acetylated in vivo abrogated the ability of the p300-IBID/SRC1 complex to activate transcription (figure 5E). These results indicate that the transcriptional activation by the p300 C-terminus -- which is supported by SRC-1 and abrogated by GRHL2 - depends upon 
acetylation of at least these (and probably other) lysines by p300 HAT, or perhaps other GRHL2sensitive HATs that remain to be determined.

A small region of GRHL2 inhibits p300 and is critical for suppressing tubulogenesis and EMT

To identify the region of GRHL2 responsible for p300 inhibition, whole domains of GRHL2 were deleted initially (figure 6A) and assayed for inhibition of HAT activity and transcriptional activation by GAL4-p300. In light of the lack of GRHL2 binding sites in most GRHL2-repressed promoters (data not shown), the surprising result was that the DNA binding domain (amino acids 245-494) was critical for inhibition (figures S15, S16). GST-GRHL2 protein sub-fragments derived from the DNA binding domain were assayed for HAT inhibition (figure 6B). The region 325-475aa, and within that, the thirteen amino acid sequence 425-437aa, inhibited HAT activity potently (figure 6C), and a synthetic peptide (aa420-442) was also able to inhibit p300 HAT activity (figure S15C). The 425-437 fragment (IRDEERKQNRKKG) is an $\alpha$ helix conserved amongst GRHL1, GRHL2, and GRHL3 (Kokoszynska et al., 2008). A GRHL2 $\Delta 425-437$ construct (with a synthetic nuclear localization signal) was unable to repress an AP-1promoter, GAL4-P300 transactivation (assayed on a GAL4-Luc reporter), the MMP1 promoter or the MMP14 promoter (figure 6D). We recently reported that GRHL2 repressed the expression of the glutamate dehydrogenase-1 gene (GLUD1), with important consequences for metabolism and anoikis (Farris et al., 2016). Interestingly, GRHL2 also repressed this promoter in a manner that required amino acids 425 to 437 (figure S17). GRHL2 $\Delta 425-437$ was still able to interact with p300 in a co-transfection assay, however (figure S18).

We hypothesized that the inhibition of p300 function could provide a mechanism for some aspects of transcriptional reprogramming by GRHL2. Consistent with the inability of the 
GRHL2 $\triangle 425-437$ to repress the AP1-dependent promoters MMP1 and MMP14 (figure 6C), this mutant was unable to suppress HGF-induced tubulogenesis in MDCK cells (figure 7A). Moreover, wild-type GRHL2 reverses EMT in Mesenchymal Subpopulation (MSP) cells, a cancer stem cell-like subpopulation derived from the mammary epithelial cell line HMLE (Cieply et al., 2012; Cieply et al., 2013). The GRHL2 $\Delta 425-437$ mutant failed to reverse EMT, by the criteria of cell morphology as well as EMT marker protein and mRNA expression (figure 7B). These results indicated that the inhibition of p300 by GRHL2 contributes significantly to transcriptional reprogramming involved in both tubulogenesis and EMT (figure 8).

\section{DISCUSSION}

Previously, we proposed that the epithelial cell is the default phenotype from both developmental and cancer biologic points of view. Under this hypothesis, the epithelial phenotype is adopted automatically by a cell in the absence of genomic/epigenetic alterations or inductive differentiation signaling (Frisch, 1997). Consistent with this idea, E-cadherinexpressing cells are the first to emerge at the late two cell stage; these eventually give rise to all differentiated cell types of the adult organism (Hyafil et al., 1980; Vestweber and Kemler, 1985; Fleming et al.,1989; Larue et al., 1994). The adenovirus-5 E1a protein, which interacts with and negatively modulates p300, enforces an epithelial phenotype, suppresses EMT, enhances anoikis, suppresses MMP expression, and exerts a ubiquitously acting tumor suppression effect in human tumor cells (Frisch and Mymryk, 2002).

The default idea has been invoked in recent studies from other laboratories, to explain, for example, the mesenchymal-epithelial transition (MET) occurring at metastatic sites (Thiery and Sleeman, 2006; Polyak and Weinberg, 2009; Tam and Weinberg, 2013). The default hypothesis could be explained molecularly if critical co-activators such as p300 were required 
for differentiation-specific but not epithelial-specific gene expression (Frisch, 1997).

Accordingly, we reported previously that GRHL2, shown here to antagonize the co-activator p300, inhibits and reverses the oncogenic EMT, suppressing tumor initiation, drug-resistance and anoikis-resistance (Cieply et al., 2012; Cieply et al., 2013).

The novel observation that GRHL2 antagonizes p300-dependent pathways in the context of tubulogenesis provides a potentially unifying mechanism for both the developmental and oncologic effects of GRHL2. GRHL2 probably regulates tubulogenesis by additional, p300independent mechanisms. For example, cells which fail to down-regulate GRHL2 appropriately may fail to down-regulate cell adhesion molecules in response to morphogenic factors (Werth et al., 2010; Senga et al., 2012). For example, E-cadherin relocalizes from cell junctions to diffuse membrane pattern in protrusive cell extensions during tubulogenesis; this relocalization might be envisioned to require GRHL2 down-regulation and subsequent loss of junctional complex components in these specific cells (Pollack et al., 1998).

p300 contributes to tubulogenesis and cancer-related EMT in multiple important ways providing a mechanism for GRHL2, via inhibition of p300, to suppress these processes. With regard to cancer, transcription factors that utilize p300/CBP and additional coactivators that interact with p300/CBP have been implicated in the oncogenic EMT, tumor drug-resistance and tumor recurrence (Matthews et al., 2007; Qin et al., 2009; Santer et al., 2011; Zhou et al., 2012; Delvecchio et al., 2013; Ringel and Wolberger, 2013; Yang et al., 2013; Xu et al., 2014; Cho et al., 2015). The upregulation of matrix metalloproteases (MMPs) associated with the oncogenic EMT requires AP-1, Ets and other p300-dependent factors (Westermarck et al., 1999; Sun et al., 2004; Chou et al., 2006; Clark et al., 2008; Lee and Partridge, 2010; Santer et al., 2011). 
With regard to tubulogenesis, the p300-dependent Ets family transcription factors Etv4 and Etv5, are key transcriptional regulators of this developmental process (Yang et al., 1998; Jayaraman et al., 1999; Goel and Janknecht, 2003; Foulds et al., 2004; Lu et al., 2009; Oh et al., 2012; Wollenick et al., 2012). In addition, the family of p300-dependent TCF/ $\beta$-catenin transcription complexes is a central hub for Wnt signaling, crucial for nephron induction (Li et al., 2007; Bridgewater et al., 2008; Miller and McCrea, 2010; Ma et al., 2012).

p300 activates transcription through an array of mechanisms; acetylation of histones, acetylation of transcription factors, the binding of the p300 bromodomain to acetylated histones to promote transcriptional activity, and recruitment of histone methyltransferases (e.g., Set1b complex) (Mizzen and Allis; Ogryzko et al., 1996; Gu and Roeder, 1997; Ito et al., 2001; Wolf et al., 2002; Stiehl et al., 2007; Chen et al., 2010; Tang et al., 2013). By inhibiting the p300 HAT activity, GRHL2 could enforce an inactive state on p300 in at least two ways. First, p300 HAT activity is substantially increased by auto-acetylation of an autoinhibitory loop on the HAT domain, which GRHL2 would suppress, stably inhibiting the enzyme (Thompson et al., 2004). Secondly, the p300 HAT domain potentiates p300 C-terminal domain (1665aa-2414aa) transactivation (Stiehl et al., 2007), which GRHL2 blocked. The minimal domain for p300 Cterminal transactivation is amino acids 2000-2180 which include the IRF-3 binding domain (IBID) (Lin et al., 2001; Matsuda et al., 2004). The p300 IBID domain cooperates with SRC1, a p160/steroid receptor coactivator family member, to activate transcription (Sheppard et al., 2001). By inhibiting the HAT activity of p300, GRHL2 suppressed co-activation by the p300-IBID complex, which was highly dependent upon the two known acetylation sites, K2086 and K2091 for activity. Interestingly, SRC1-p300 has been shown previously to induce TWIST expression and EMT in breast cancer (Qin et al., 2009), and GRHL2 is a potent EMT suppressor. 
Although more than four hundred DNA binding proteins interact with p300 and utilize it as a co-activator, the inhibitory effect of GRHL2 on p300 is nearly unique, shared by only one other family of proteins called the E1a-like inhibitor of differentiation (EID) proteins. EID1 inhibits p300 HAT activity (MacLellan et al., 2000) and EID3 blocks the SRC-1/CBP interaction, while the mechanism of EID2 is controversial (Ji et al., 2003; Miyake et al., 2003; Båvner et al., 2005). The effects of EID proteins upon EMT or tubulogenesis have not yet been investigated to our knowledge.

GRHL2 regulates intracellular metabolism and this could indirectly affect p300 by altering the levels of the co-factors for p300 HAT, acetyl-CoA or crotonyl-CoA ( $\mathrm{Li}$ and Li, 2015; Farris et al., 2016). This may provide an additional, more indirect mechanism for GRHL2 to affect p300 function.

In summary, p300 and related co-activators are crucial for cells to differentiate. Under the default-phenotype hypothesis, this can be extended to critical roles in EMT, in contexts such as tubulogenesis and oncogenesis. The inhibition of p300 by GRHL2 provides a mechanism for GRHL2 to enforce the default epithelial phenotype.

\section{MATERIALS AND METHODS}

\section{Cell lines}

293 HEK cells without T antigen (Doug Black, UCLA, Los Angeles), MDCK cells (Clone M8 described previously in Frisch et al. (Frisch and Francis, 1994), and HT1080 cells (ATCC) were cultured in advanced Dulbecco's Modified Eagle’s Medium (DMEM) (Gibco) with 10\% fetal bovine serum (Hyclone) and 1x penicillin-streptomycin-glutamine (PSG) (Invitrogen). miMCD- 
3 cells (ATCC) cultured in advanced DMEM:Ham's F12 (Gibco) with 10\% fetal bovine serum and 1x PSG. HMLE and HMLE MSP cells were described previously (Cieply et al., 2013).

Generation of stable cell lines by retroviral transduction and lentiviral transduction

Full-length GRHL2/pMXS-IRES-Puro and retroviral packaging protocol were described previously (Farris et al., 2016). GRHL2 $\Delta 425-437$ was subcloned into pMXS-IRES-Puro from GRHL2 $\Delta 425-437-N L S-3 x F L A G / p C D N A 3.1+$. GRHL2 shRNA/pGipZ and scramble control shRNA/pGipz were obtained from Open Biosystems and packaged as previously described (Cieply et al., 2012). The shRNA construct in pTRIPZ was made by subcloning a MluI-XbaI fragment from pGIPZ.

\section{Cell Scattering Assays}

MDCK cells were plated on collagen coated 6 well dishes to give 25\% cell confluency on HGF induction. Cells were treated with 60ng/ml recombinant human HGF (RD Systems) in DMEM media or fresh DMEM media for the time periods indicated. The cells were imaged and then harvested for protein or RNA analysis. Images were obtained using a Zeiss Axiovert 200M, Axiocam MRM camera, Phase 20x/0.55, RT, Axiovision Rel. 4.8 software. Cell scattering time lapse movies were taken every 15 minutes over a 24 hour period. Time lapse images were obtained using a Nikon Eclipse TE2000-E with Photometrics CoolSNAP HQ2 Monochrome CCD with Phase 40x/0.75 objective using Bioptechs Delta T4 Culture Dish Heater, Prior ProScan II Encoded Motorized Stage, and OKO LABS Digital Stage Top Incubator (37 degrees and $5 \% \mathrm{CO}_{2}$ ). For each data point, four 40x images were stitched together using $10 \%$ overlap with background correction in NIS-Elements AR Nikon. 


\section{Western Blotting}

Protein lysates were incubated at 95 degrees for 5 minutes in 1 X SDS lysis buffer (125mM Tris$\mathrm{HCl}$, pH 6.8, 0.04\% SDS, 0.024\% bromophenol blue, 5\% $\beta$-mercapatoethanol, and 20\% glycerol). SDS-Page was run in 4\%-20\% gradient Novex Tris-Glycine gel (Invitrogen). Proteins were electrophoretically transferred to a polyvinylidene difluoride membrane (Immobilon) in Tris-Glycine buffer with 5\% methanol. Membranes were blocked for 1 hour in 1xTBS $+5 \%$ nonfat milk. Primary and secondary antibodies were incubated in $1 x T B S+5 \%$ nonfat milk $+0.1 \%$ Tween-20. Primary antibodies were incubated for 2 hour/RT or overnight/4 degrees at 1:1000 dilution. Primary antibodies used were: GRHL2, rb (Sigma); $\beta$-actin, ms (Thermo-pierce); Fibronectin, ms (BD Biosciences); Vimentin, ms ((Santa Cruz Bio-tech [SCBT]); $\beta$-actin, ms (Sigma); E-cadherin, ms (BD Biosciences); $\beta$-catenin, ms (BD Biosciences); GAPDH, ms (Origene); acetyl-lysine, rb (Millipore); H3K18AC, rb (Cell Signaling [CS]); H3K27AC, rb (CS); total H3, rb (CS); GST, ms (Thermo-pierce); p300,rb (SCBT sc-584); p300, rb (SCBT sc-585); FLAG, ms (Sigma); GAL4, rb (SCBT). Goat antirabbit or anti-mouse horseradish peroxidase conjugated secondary antibodies (Bio-rad) were used at 1:3000 dilution and incubated for 1hour/RT. Western blot membranes were developed using ECL-West Pico (Pierce) and analyzed using standard chemiluminescence techniques.

\section{MDCK Tubulogenesis assay}

MDCK Tubulogenesis assay was performed as previously described (Elia and LippincottSchwartz, 2001). Briefly, collagen matrix was made by mixing 10x MEM (Invitrogen), 100x PSG, $23.5 \mathrm{~g} / \mathrm{L} \mathrm{NaHCO}_{3}$ (Invitrogen), sterile water, and HEPES (Invitrogen) to working concentrations. Vitrogen Bovine Collagen (Advanced Biomatrix 5005-b) was added to a final concentration of 2 
$\mathrm{mg} / \mathrm{mL}$. $\mathrm{NaHCO}_{3}$ was used to bring the solution to $\mathrm{pH} 7.3$ via $\mathrm{pH}$ strip indicator (Elia and Lippincott-Schwartz, 2001). In a 24-well plate, $100 \mu$ l of working collagen solution was placed in a 1.0 micron pore cell culture insert (Falcon) and allowed to solidify for 3 hours in tissue culture incubator. Sub-confluent MDCK cells were trypsinized and counted using Countess Cell counting machine (Invitrogen). Cells were pipetted into the working collagen solution to give a final concentration of 5,000 cells/ml. Then, $250 \mu \mathrm{l}$ of cell/collagen solution was pipetted onto the presolidified collagen matrix in the cell culture insert. The cell-collagen matrix was allowed to solidify for 2 hours at 37 degrees and $5 \% \mathrm{CO}_{2}$ and then $250 \mu \mathrm{l}$ and $500 \mu \mathrm{l}$ of media were place on cell-collagen matrix cell culture insert and in the 24 well, respectively. Media were changed every 3 days. After 6 days, $60 \mathrm{ng} / \mathrm{ml}$ recombinant human HGF was added to indicated wells, and branching morphogenesis was monitored by microscopy. MDCK cyst staining was performed as previously described (Elia and Lippincott-Schwartz, 2001), with the only modification being the substitution of 7mg/mL fish skin gelatin for Triton X-100 in permeabilization buffer. Cysts were stained with Alexa Fluor 488 Phalloidin (Molecular Probes) and Hoescht 33258 (Molecular Probes). Representative 2 day post-HGF images were obtained using a Zeiss 710 Confocal with Airyscan, C-Apochromat 40x/1.2 W Corr, room temperature, AxioObserver.Z1 software, and Diode 405 and Argon 488 lasers. Images were processed in Adobe Photoshop. Tubulogenesis was observed by day 2 post-HGF addition and quantified on day 5 post-HGF addition.

RNA-Seq Assay

MDCK+GRHL2/pMXS and MDCK+shGRHL2/Gipz cells were plated in collagen-coated 60mm2 dishes to obtained 25\% confluency upon HGF treatment. Cells were treated with either 60ng/ml HGF in DMEM complete or DMEM complete media alone for 24 hours; RNA was isolated from all four treatment conditions in triplicate using the RNeasy Plus Kit (Qiagen) and 
quantified using Nandrop (Fischer). Raw data were aligned to the CanFam3 reference genome using TopHat2 (Kim et al., 2013). For each sample and each gene, the number of reads generated from that sample and mapping to that gene were counted using RSamtools. Genes were as defined in the ensembl database v82 (Cunningham et al., 2015). The resulting count table was analyzed using DESeq (Anders and Huber, 2010), performing two independent comparisons: samples with HGF compared to those without, with GRHL2 overexpressed, and, samples with HGF compared to those without, both expressing GRHL2 shRNA. Genes were considered differentially expressed if they showed a false discovery rate of 0.05 or less and a fold change of 1.5 or higher in either direction. Genes found to be regulated by HGF with shRNA against GRHL2, but not found to be regulated by HGF with GRHL2 overexpressed were uploaded to Ingenuity Pathway Analysis (Qiagen), where a Core Analysis was performed using default settings.

Quantitative reverse transcription PCR analysis

MDCK parent, GRHL2/pMXS, and shGRHL2/pGipZ cells were plated on collagen-coated $60 \mathrm{~mm}^{2}$ dishes and treated as indicated. Cells were harvested using RNAeasy Plus mini kit (Qiagen), analyzed using Nanodrop, and converted to cDNA using SuperScript III First-Strand Synthesis SuperMix (Invitrogen) with oligo dT primers and 1ug of RNA. cDNA was analyzed using SYBR Green PCR Master Mix on an Applied Biosystems 7500 Real Time PCR System. Values reported were determined using the delta delta CT method using canine CBX1 as internal control. Primers were as follows: CBX1-F (AAGTATTGCAAGGCCACCCA), CBX-R (TTTTCCCAATCAGGCCCCAA), GRHL2-F (TTCCTCCCCAGGAGAGATGG), GRHL2-R (GGCCCAACACACGGATAAGA), MMP1-F (TGACTGGAAAGGTCGACACG), MMP1-R (GACCTTGGTGAAGGTCAGGG), MMP3-F (CTGACCCTAGCGCTCTGATG), MMP3-R 
(GTCCTGAGGGATTTTCGCCA ), MMP13-F (GGACTTCCCAGGGATTGGTG), MMP13-R

(ATGACACGGACAATCCGCTT), MMP14-F (CCCAGAGGAGGGCATGTTTT), MMP14-R

(GACTCCCCACCCTTCCAATG), FOS-F (GGGAGGACCTTATCTGTGCG), FOS-R

(ACACACTCCATGCGTTTTGC), FOSL1-F (ACACCCTCCCTGACTCCTTT), FOSL1-R

(CTGAAGAGGGCATGGGATTA), FOSB-F (GGAGAAGAGAAGGGTTCGCC), FOSB-R

(CACAAACTCCAGACGCTCCT), JUN-F (GACCTTCTACGACGATGCCC), JUN-R

(TTCTTGGGGCACAGGAACTG), JUNB-F (GTGAAGACACTCAAGGCCGA), and JUNB-R (AGACTGGGGGAATGTCCGTA).

Luciferase Reporter Assays

HT1080 or 293 NOT cells were transiently transfected using Lipofectamine 2000 (Invitrogen) at a $1 \mu$ g DNA: $3 \mu$ l Lipofectamine ratio. After 20 minute incubation in $300 \mu \mathrm{l}$ of Opti-MEM (Invitrogen), a total of $1.0 \mu \mathrm{g}$ of DNA was transfected into a 12 well with DMEM media and refeed 4 hours later with DMEM media. After 28-36 hours post-transfection, the cells were washed twice with cold PBS, lysed in $200 \mu \mathrm{l}$ of 1x Cell Culture Lysis Buffer (Promega), frozen for at least 1 hour at -80 degrees, thawed on ice, and centrifuged at 13,200rpm. The supernatants were assayed for luciferase activity (Promega) and $\beta$-galactosidase activity as internal control (2x $\beta$-galactosidase assay reagent, $200 \mathrm{mmol} / \mathrm{l}$ sodium phosphate, $\mathrm{pH} 7.3,2 \mathrm{mmol} / \mathrm{L} \mathrm{MgCl}_{2}$, 100mmol/L 2-mercaptoethanol, and 1.33mg/mL o-nitrophenylgalactoside). MMP1 promoter (-1200 to +60) and MMP14 promoter (-1114 to -242) luciferase reporter plasmids were generated by subcloning the promoter from genomic DNA using PCR into the pGL3-basic luciferase reporter plasmid. Primer sequences were: MMP1prom-F (TTATTACTCGAGCCCTCCCTCTGATGCCTCTGA), MMP1prom-R (TATATAAAGCTTCTCCAATATCCCAGCTAGGAA), MMP14prom-f 
(TTATTACTCGAGCCCTTTCTCACGGTCAGCTT), MMP14prom-R

(TATATAAAGCTTTCTCGCTCTCTCCTCTGGTC). GRHL2/pcDNA3.1, TK-LacZ and

E1a/pCDNA3.1 plasmids were described previously (Grooteclaes and Frisch, 2000; Cieply et al., 2012). E1A p300 binding mutant was a generous gift from Arnold Berk (UCLA, Los Angeles, California) subcloned into pCDNA3.1+. GRHL2 $\Delta 425-437$ was generated by cloning GRHL2 1-424aa and GRHL2 438-625aa fragments and ligating together with Bglll and then ligating into pCDNA3.1+ using BamHI and Sal1. Primers used were GRHL2-1-424-F (TATATAGGATCCATGTCACAAGAGTCGGACAA), GRHL2-1-424-R (ATATAAAGATCTTTTTCTTTCTGCTCCTTTGT), GRHL2-438-625-F (TAAATTAGATCTAAAGGCCAGGCCTCCCAAAC), GRHL2-438-625-R (TTATATGTCGACCTAGATTTCCATGAGCGTGA). pGL4.44[luc2P/AP1 RE/Hygro] (Promega) was used to assay for AP-1 responsive promoter activity and control plasmid was pGL4.27 (Promega). Phorhol 12-myristate 13-acetate (PMA) induction (10ng/mL) (ThermoFisher) was used for assaying AP-1 activity in tranfected 293 cells. Full length p300GAL4/VR1012 and EV-GAL4/VR1012 were generous gifts from Neil Perkins (Newcastle University, UK); they were assayed on the GAL4 responsive reporter pG5-Luc (Promega). GAL4- p300 HAT (1283-1674aa), GAL4- p300C-terminus (1665-2414aa), and GAL4- p300 IBID (2050-2096) were generated by subcloning PCR fragments (MluI-NotI) into pBIND (Promega). Primer sequences were: p300 c-terminal-F (TATATAACGCGTATCGCTTTGTCTACACCTGCAAT), p300 c-terminal-R (TTTAAAGCGGCCGCCTAGTGTATGTCTAGTGTAC), p300 HAT-F (TATATAACGCGTATAGGAAAGAAAATAAGTTTTCT), p300 HAT-R (TTTAAAGCGGCCGCCTAGTCCTGGCTCTGCGTGTGCAG), p300 IBID-F 
(TATATAACGCGTATGCCTTACAAAACCTTTTGCG), and p300 IBID-R

(TTTAAAGCGGCCGCCTAATTAGAGTTGGCATACTTGG). To generate the p300-IBID Mutant (K2086A and K2091A), the p300 IBID lysine nucleotide sequences were mutated to alanine and flanking Mlu1 and Not1 restriction enzyme were added to the 5' and 3' ends, respectively. The sequence was then generated as a gBlock gene fragment (IDT). The p300 mutant sequence was 5' CACAACCAGG ATTGGGCCAG GTAGGTATCA GCCCACTCGC ACCAGGCACT GTGTCTCAAC AAGCCTTACA AAACCTTTTG CGGACTCTCA GGTCTCCCAG CTCTCCCCTG CAGCAGCAAC AGGTGCTTAG TATCCTTCAC GCCAACCCCC AGCTGTTGGC TGCATTCATC GCGCAGCGGG CTGCCGCGTA TGCCAACTCT AATCCACAAC 3". The IBID mutant fragment was digested and ligated into pBIND vector. HA-SRC1/pACTAG was a generous gift from Timothy Beischlag (Simon Fraser University, Canada).

\section{Immunoprecipitation}

GRHL2-STAP contained full-length human GRHL2 fused to a C-terminal myc-Streptavidin Binding Peptide-S-Tag sequence derived by ligating a synthetic oligonucleotide containing the S-tag to a PCR product containing the myc-SBP tags from the pCeMM-CTAP vector described (Burckstummer et al., 2006). 293 NOT cells were plated on collagen coated 6 well dishes and transfected using $1 \mu \mathrm{g}$ of empty vector/pCDNA3.1+ or GRHL2-S-TAP/pCDNA3.1+ and 1ug of empty vector/VR1012 or p300/VR1012) with 6ul of lipofectamine (Invitrogen) in 300ul of OptiMEM (Invitrogen). Cells were incubated for 4-6 hours with DMEM media, and then refed with DMEM media. After 28 hours post-transfection, cells were lysed in 600 $\mu$ l of cold p300 Lysis Buffer (25mM Tris-HCl pH8, 75mM NaCl, 0.5mM EDTA, 10\% glycerol, 0.1\% NP40, and protease inhibitor tablet (Roche)). Sample lysates were passed through $1 \mathrm{ml}$ syringe with $27 \mathrm{~g}$ 
needle 3 times and precleared in 4 degree microcentrifuge at 13,200rpm for 10 minutes. Total lysate samples were obtained, mixed 1:1 with 2X SDS Lysis buffer (250mM Tris-HCl, pH 6.8, 0.08\% SDS, $0.048 \%$ bromophenol blue, $10 \% \beta$-mercapatoethanol, $40 \%$ glycerol) and heated for 5 minutes at 95 degrees. Lysates were precipitated for 2 hours with 40 ul of a $50 \%$ of S proteinagarose (EMD Millipore) which had been pre-equilibrated with p300 lysis buffer containing 10mg/ml BSA. Immunoprecipitation lysates were washed three times with p300 lysis buffer, diluted with 2X SDS lysis buffer, and heated for 5 minutes at 95 degrees. Lysates were processed using previously described western blotting methods. For endogenous p300 and ectopic GRHL2 co-immunoprecipitation, MSP cells with empty vector/pMXS or GRHL2/pMXS were used in the experiment. For endogenous p300 and endogenous GRHL2 coimmunoprecipitation, parental HMLE cells were used in the experiment. The coimmunoprecipitation method was identical between the different cell lines. For each cell line, 2 100mm dishes were grown to $80 \%$ confluency, washed twice with cold PBS, lysed in $1 \mathrm{~mL}$ of p300 lysis Buffer with protease inhibitors, and passed through 27g needle three times. After lysates were precleared at 13,200rp, 10 $\mu$ g of p300 antibody (SCBT, SC-584) or rabbit IgG (Jackson) was added to lysates and incubated overnight on 4 degree wheel. Pre-equilbrated Protein A-Sepharose (45 $\mu \mathrm{l}$ ) (GE Healthcare) was added and incubated for 90 minutes. Samples were washed 3 times in p300 lysis buffer, added 45ul of 2X SDS lysis buffer, and heated at 95 degrees for 5 minutes. Lysates were processed using previously described western blotting methods.

\section{Histone Acetylation Assays}

Histone acetylation reactions contained indicated amounts of recombinant baculovirus flagtagged p300 (37.5ng) (Active Motif) or baculovirus p300 HAT (1283-1673) (Sigma), 250ng of 
H3 (Cayman), $100 \mu \mathrm{M}$ acetyl-CoA (MP Biomedical), and indicated amounts of prescission cleaved GRHL2, GST-GRHL2 or GST alone in 30 $\mu$ l of p300 acetylation buffer (50mM Tris$\mathrm{HCl} \mathrm{pH} \mathrm{8.0,1mM} \mathrm{DTT,} \mathrm{0.1mM} \mathrm{EDTA,} \mathrm{10 \%} \mathrm{glycerol} \mathrm{and} \mathrm{protease} \mathrm{inhibitor).} \mathrm{Acetylation}$ reactions were incubated for 15 minutes at 30 degrees on heat block. Reactions were diluted with 2x SDS Lysis buffer and incubated for 5 minutes at 95 degrees. Lysates were processed using previously described western blotting methods.

\section{Recombinant protein purification}

Full length GRHL2 was subcloned into pGEX-6P-3 vector (GE Healthcare) and transformed in to BL21 bacteria (Invitrogen). GRHL2 fragments were subcloned into pGEX-6p-3 using BamH1 and Sal1. A colony was selected and grown in 20mL of LB+amp overnight at 37 degrees. The following day, $10 \mathrm{~mL}$ of culture was inoculated into $500 \mathrm{~mL}$ of LB+amp and incubated for approximately 2 hours at 37 degrees with shaking until OD 0.6 was obtained. IPTG was added to final concentration of $0.1 \mathrm{mM}$ and incubated overnight on shaker at room temperature. The bacteria were spun in chilled centrifuge for 10 minutes at 5000rpm. Bacterial pellet was resuspended in $10 \mathrm{~mL}$ of BL21 lysis buffer (1xPBS, 1\% Triton-100, 1mM DTT, 0.4\% lysozyme and protease inhibitor tablet), incubated for 10 minutes, and sonicated twice for 30 seconds. The suspension was cleared at 13,000 rpm for 10 minutes. To the supernatant, $150 \mu \mathrm{L}$ of 50\% glutathione-sepharose beads (GE Healthcare) was added and incubated for 2 hours with rotation at 4 degrees. Glutathione-sepharose beads were washed three times with glutathione washing buffer (1xPBS, 1mM DTT and 0.1\% Triton-X) and eluted for 30 minutes with glutathione elution buffer (100mM Tris pH 8, 150mM NaCl, 20mM Glutathione, and protease inhibitors). Alternatively, glutathione-sepharose beads with GRHL2 protein attached were washed 3 times in PreScission cleavage buffer (50mM Tris-HCl, 150mM NaCl, 1mM EDTA, 
1mM DTT, pH 7.0). The GST-GRHL2 on glutathione-sepharose beads was cleaved by adding 80ul of Prescission Protease (GE Healthcare) per mL of PreScission cleavage buffer at 5 degrees overnight. The sample was spun at 2,000rpm and the supernatant with the GST cleaved GRHL2 was obtained. Proteins were dialyzed against p300 acetylation buffer overnight at 4 degrees using 20,000 MWCO Slide-A-Lyzer MINI Dialysis Unit (Thermo-Scientific). Protein was analyzed on SDS-PAGE by Coomassie Blue staining and quantified by comparison of Coomassie staining to a BSA standard and/or a BCA assay. Synthetic peptides were purchased from ThermoFischer Scientific with n-terminus acetyl group and c-terminus amidation: GRHL2 420-442aa (GAERKIRDEERKQNRKKGKGQAS) and scramble (IDEKNKGRERQRK)

\section{Chromatin Immunoprecipitation (ChIP)}

For ChIP assay, HT1080 cell lines expressing empty vector/pMXS or GRHL2/pMXS cells were used, and chromatin generated as described previously with the following modifications (Kumar et al.): (i) Immunopreciptation was performed with 3ug of H3K27-Ac antibody (Cell Signaling) or rabbit IgG (Jackson) to $3.3 \times 10^{6}$ cell-equivalents in ChIP RIPA buffer. (ii) Protein A-sepharose was used instead of FLAG-agarose beads. (iii) qPCRs were performed using 2X SYBR green master mix (Applied Biosystems; $10 \mathrm{uL}$ ), $0.08 \mathrm{uL}$ of 100 micromolar primer, $1 \mathrm{uL}$ ChIP DNA in a $20 \mathrm{uL}$ reaction. Primer sequences were as follows: MMP1-1-F

(AACCTCAGAGAACCCCGAAG), MMP1-1-R (TACTAACACTGCGCACCTGA) MMP1-2F (CAGAGTGGGGCATGAGTAGG), MMP1-2-R (TGTCTTCGGTACTGGTGACC), MMP14-F (AAGTAAGTGAGCTTCCCGGC), MMP14-R (GGAGTTCGCCCCAGTTGTAA), MMP2-F (TCGCCCATCATCAAGTTCCC), MMP2-R (CCCCCAAGCTGTTTACCGAA), ZEB1-1-F (GCGAGGCGTGGGACTGATGG), ZEB1-1-R (AAAGTTGGAGGCTCGGCGGC), ZEB1-2-F (CTGCACGGCGATGACCGCT), ZEB1-2-R (TTCCGCTTGCCAGCAGCCTC), 
CDH1-F (ACTCCAGGCTAGAGGGTCACC), CHD1-R

(CCGCAAGCTCACAGCTTTGCAGTTCC), ESRP1-F (GGGAGCTTGGTCAAGTCAAC), ESRP1-R (TCTTAAATCGGGCCACGCAG), RAB25-F (AGGTCCTGTCCCTTTTTCGC), RAB25-R (TTGGGGGTAAGGGGACTTCT), GAPDH-F (ATGGTTGCCACTGGGGATCT), and GAPDH-R (TGCCAAAGCCTAGGGGAAGA). Results were normalized to GAPDH values.

Ex Vivo Kidney Culture

All experiments involving mice were approved by WVU IACUC. Hoxb7creEGFP transgenic mice and ex vivo kidney culture method were described previously (Zhao et al., 2004). We removed embryonic kidney at E13.5 and the kidneys were cultured in 1uM batimastat (Tocris) or vector in culture media for 48 hours. Images were taken of GFP ureteric buds on Olympus MVX10 Macro Zoom equipped with an ORCA-Flash 4.0 camera, using 2x objective and 4x zoom 4x, FITC, and using CellSen Imaging Software. Ureteric buds were counted for quantification.

Confocal microscopy of transfected RRE.1 cells

RRE.1 cells, wt e1a-NLS-LacI-mCherry, and empty vector NLS-LacI-mCherry were generous gift from Arnold Berk (UCLA, Los Angeles) and experiment was performed as previously described (Verschure et al., 2005; Ferrari et al., 2014). GRHL2 was subcloned into empty vector NLS-LacI-mCherry using Sal1. Images were taken on Zeiss Axiolmager Z1 microscope LSM 510 Confocal, 63x/0.75 LDPlan-neofluar, DAPI and Rhodamine, and Zeiss Zen Software. Images were quantified using mean area on Image J. 


\section{Statistical Analysis}

Error bars in graphs represent standard deviation. P-values were calculated using a Student's two-tailed t-test.

\section{ACKNOWLEDGMENTS}

The authors would like to thank Dr. Neil Perkins (University of Newcastle, UK), Dr. Arnold Berk (UCLA) and Dr. Stephen Jane (Monash University, Australia) for constructs and technical advice, Dr. Kathy Brundage for flow cytometry, Dr. Karen Martin and Dr. Amanda Ammer for imaging expertise, Dr. Ryan Percifield for assistance in RNA library construction, Dr. Neil Infante for bioinformatics support, Dr. Peter Mathers, Sarah McLaughlin and Emily Ellis for animal management, Dr. Paolo Fagone for protein preparation, Dr. Carlton Bates and Dr. Kenneth Walker for kidney development techniques, and Dr. Mary Davis for Ingenuity Pathway Analysis. The work was supported by a grant from the Mary Kay Foundation and a grant from the National Institute Of General Medical Sciences, U54GM104942. Dr. James Denvir was supported in part by the West Virginia IDeA Network for Biomedical Research Excellence (NIH/NIGMS P20GM103434). The following NIH grants supported the flow cytometry facility: GM103488/RR032138; RR020866;OD016165;GM103434. AMIF: “Small animal imaging and image analysis were performed in the West Virginia University Animal Models \& Imaging Facility, which has been supported by the Mary Babb Randolph Cancer Center and NIH grants P20 RR016440, P30 GM103488 and S10 RR026378.” MIF: “Imaging experiments and image analysis were performed in the West Virginia University Microscope Imaging Facility, which has been supported by the Mary Babb Randolph Cancer Center and NIH grants P20 RR016440, P30 GM103488 and P20 GM103434.” 
Table 1.

Genes regulated by GRHL2

Gene ID

Fold Change

Significance

(shGRHL2+HGF/GRHL2+HGF)

\begin{tabular}{lll}
\hline MMP-1 & 31.7 & $\begin{array}{l}\text { Interstitial collagenase, necessary for MDCK } \\
\text { tubulogenesis, p300-dependent expression }\end{array}$ \\
MMP-9 & 5.8 & $\begin{array}{l}\text { Gelatinase B, p300-dependent cancer } \\
\text { invasiveness, upregulated in EMT }\end{array}$ \\
& 15.1 & Collagenase 3, Necessary for MDCK \\
MMP-13 & 5.82 & tubulogenesis, p300-dependent expression \\
& & Membrane-type matrix metalloprotease, involved \\
MMP-14 & & in EMT regulation and invasiveness phenotype \\
& 3.5 & Increases invasion in multiple cancers \\
MMP-19 & 9.6 & \\
\hline MMP-24 & & \\
\hline
\end{tabular}

Table 1- HGF-induced MMPs expression in absence of GRHL2 compared to the presence of GRHL2 


\section{References}

Anders, S., and Huber, W. (2010). Differential expression analysis for sequence count data. Genome Biol 11, R106.

Auden, A., Caddy, J., Wilanowski, T., Ting, S.B., Cunningham, J.M., and Jane, S.M. (2006). Spatial and temporal expression of the Grainyhead-like transcription factor family during murine development. Gene Expr Patterns 6, 964-970.

Aue, A., Hinze, C., Walentin, K., Ruffert, J., Yurtdas, Y., Werth, M., Chen, W., Rabien, A., Kilic, E., Schulzke, J.-D., Schumann, M., and Schmidt-Ott, K.M. (2015). A Grainyhead-Like 2/Ovo-Like 2 Pathway Regulates Renal Epithelial Barrier Function and Lumen Expansion. J Am Soc Nephrol 26, 2704-2715.

Båvner, A., Matthews, J., Sanyal, S., Gustafsson, J.-Å., and Treuter, E. (2005). EID3 is a novel EID family member and an inhibitor of CBP-dependent co-activation. Nucleic Acids Res 33, 3561-3569.

Bedford, D.C., Kasper, L.H., Fukuyama, T., and Brindle, P.K. (2010). Target gene context influences the transcriptional requirement for the KAT3 family of CBP and p300 histone acetyltransferases. Epigenetics 5, 9-15.

Benbow, U., and Brinckerhoff, C.E. (1997). The AP-1 site and MMP gene regulation: What is all the fuss about? Matrix Biol 15, 519-526.

Blobel, G.A. (2000). CREB-binding protein and p300: molecular integrators of hematopoietic transcription. Blood 95, 745-755.

Boglev, Y., Wilanowski, T., Caddy, J., Parekh, V., Auden, A., Darido, C., Hislop, N.R., Cangkrama, M., Ting, S.B., and Jane, S.M. (2011). The unique and cooperative roles of the Grainy head-like transcription factors in epidermal development reflect unexpected target gene specificity. Dev Biol 349, 512-522. 
Bridgewater, D., Cox, B., Cain, J., Lau, A., Athaide, V., Gill, P.S., Kuure, S., Sainio, K., and Rosenblum, N.D. (2008). Canonical WNT/ $\beta$-catenin signaling is required for ureteric branching. Dev Biol 317, 83-94.

Burckstummer, T., Bennett, K.L., Preradovic, A., Schutze, G., Hantschel, O., Superti-Furga, G., and Bauch, A. (2006). An efficient tandem affinity purification procedure for interaction proteomics in mammalian cells. Nat Methods 3, 1013-1019.

Chacon-Heszele, M.F., Zuo, X., Hellman, N.E., McKenna, S., Choi, S.Y., Huang, L., Tobias, J.W., Park, K.M., and Lipschutz, J.H. (2014). Novel MAPK-dependent and -independent tubulogenes identified via microarray analysis of 3D-cultured Madin-Darby canine kidney cells. Am J Physiol Renal Physiol 306, F1047-F1058.

Chen, J., Ghazawi, F.M., and Li, Q. (2010). Interplay of bromodomain and histone acetylation in the regulation of p300-dependent genes. Epigenetics 5, 509-515.

Cho, M.H., Park, J.H., Choi, H.J., Park, M.K., Won, H.Y., Park, Y.J., Lee, C.H., Oh, S.H., Song, Y.S., Kim, H.S., Oh, Y.H., Lee, J.Y., and Kong, G. (2015). DOT1L cooperates with the c-Mycp300 complex to epigenetically derepress CDH1 transcription factors in breast cancer progression. Nat Commun 6, 7821.

Chou, Y.T., Wang, H., Chen, Y., Danielpour, D., and Yang, Y.C. (2006). Cited2 modulates TGF-[beta]-mediated upregulation of MMP9. Oncogene 25, 5547-5560.

Cieply, B., Farris, J., Denvir, J., Ford, H.L., and Frisch, S.M. (2013). Epithelial-Mesenchymal Transition and Tumor Suppression Are Controlled by a Reciprocal Feedback Loop between ZEB1 and Grainyhead-like-2. Cancer Res 73, 6299-6309. 
Cieply, B., Riley, P.t., Pifer, P.M., Widmeyer, J., Addison, J.B., Ivanov, A.V., Denvir, J., and Frisch, S.M. (2012). Suppression of the Epithelial-Mesenchymal Transition by Grainyhead-like2. Cancer Res 72, 2440-2453.

Clark, I.M., Swingler, T.E., Sampieri, C.L., and Edwards, D.R. (2008). The regulation of matrix metalloproteinases and their inhibitors. Int J Biochem Cell Biol.40, 1362-1378.

Cunningham, F., Amode, M.R., Barrell, D., Beal, K., Billis, K., Brent, S., Carvalho-Silva, D., Clapham, P., Coates, G., Fitzgerald, S., Gil, L., Girón, C.G., Gordon, L., Hourlier, T., Hunt, S.E., Janacek, S.H., Johnson, N., Juettemann, T., Kähäri, A.K., Keenan, S., Martin, F.J., Maurel, T., McLaren, W., Murphy, D.N., Nag, R., Overduin, B., Parker, A., Patricio, M., Perry, E., Pignatelli, M., Riat, H.S., Sheppard, D., Taylor, K., Thormann, A., Vullo, A., Wilder, S.P., Zadissa, A., Aken, B.L., Birney, E., Harrow, J., Kinsella, R., Muffato, M., Ruffier, M., Searle, S.M.J., Spudich, G., Trevanion, S.J., Yates, A., Zerbino, D.R., and Flicek, P. (2015). Ensembl 2015. Nucleic Acids Res 43, D662-D669.

Delvecchio, M., Gaucher, J., Aguilar-Gurrieri, C., Ortega, E., and Panne, D. (2013). Structure of the p300 catalytic core and implications for chromatin targeting and HAT regulation. Nat Struct Mol Biol 20, 1040-1046.

Elia, N., and Lippincott-Schwartz, J. (2009). Culturing MDCK Cells in Three Dimensions for Analyzing Intracellular Dynamics. In: Curr Protoc Cell Biol: John Wiley \& Sons, Inc.

Farris, J.C., Pifer, P.M., Zheng, L., Gottlieb, E., Denvir, J., and Frisch, S.M. (2016). Grainyheadlike 2 Reverses the Metabolic Changes Induced by the Oncogenic Epithelial-mesenchymal Transition: Effects on Anoikis. Mol Cancer Res

Ferrari, R., Gou, D., Jawdekar, G., Johnson, S.A., Nava, M., Su, T., Yousef, A.F., Zemke, N.R., Pellegrini, M., Kurdistani, S.K., and Berk, A.J. (2014). Adenovirus Small E1A Employs the Lysine Acetylases p300/CBP and Tumor Suppressor Rb to Repress Select Host Genes and Promote Productive Virus Infection. Cell Host \& Microbe 16, 663-676. 
Foulds, C.E., Nelson, M.L., Blaszczak, A.G., and Graves, B.J. (2004). Ras/Mitogen-Activated Protein Kinase Signaling Activates Ets-1 and Ets-2 by CBP/p300 Recruitment. Mol Cell Biol 24, 10954-10964.

Fleming, T. , McConnell, J., Johnson, MH., and Stevenson BR. (1989). Development of tight junctions de novo in the mouse early embryo: control of assembly of the tight junction-specific protein, ZO-1. J Cell Biol 108, 1407-1418.

Frisch, S.M. (1997). The epithelial cell default-phenotype hypothesis and its implications for cancer. Bioessays 19, 705-709.

Frisch, S.M., and Francis, H. (1994). Disruption of epithelial cell-matrix interactions induces apoptosis. J Cell Biol 124, 619-626.

Frisch, S.M., and Mymryk, J.S. (2002). Adenovirus-5 E1A: paradox and paradigm. Nat Rev Mol Cell Biol 3, 441-452.

Gao, X., Vockley, C.M., Pauli, F., Newberry, K.M., Xue, Y., Randell, S.H., Reddy, T.E., and Hogan, B.L. (2013). Evidence for multiple roles for grainyhead-like 2 in the establishment and maintenance of human mucociliary airway epithelium.[corrected]. Proc Natl Acad Sci U S A 110, 9356-9361.

Goel, A., and Janknecht, R. (2003). Acetylation-Mediated Transcriptional Activation of the ETS Protein ER81 by p300, P/CAF, and HER2/Neu. Molecular and Cellular Biology 23, 6243-6254. Grooteclaes, M.L., and Frisch, S.M. (2000). Evidence for a function of CtBP in epithelial gene regulation and anoikis. Oncogene 19, 3823-3828.

Gu, W., and Roeder, R.G. (1997). Activation of p53 Sequence-Specific DNA Binding by Acetylation of the p53 C-Terminal Domain. Cell 90, 595-606. 
Gustavsson, P., Copp, A.J., and Greene, N.D. (2008). Grainyhead genes and mammalian neural tube closure. Birth Defects Res A Clin Mol Teratol 82, 728-735.

Harrison, M.M., Botchan, M.R., and Cline, T.W. (2010). Grainyhead and Zelda compete for binding to the promoters of the earliest-expressed Drosophila genes. Dev Biol 345, 248-255.

Hellman, N.E., Spector, J., Robinson, J., Zuo, X., Saunier, S., Antignac, C., Tobias, J.W., and Lipschutz, J.H. (2008). Matrix Metalloproteinase 13 (MMP13) and Tissue Inhibitor of Matrix Metalloproteinase 1 (TIMP1), Regulated by the MAPK Pathway, Are Both Necessary for Madin-Darby Canine Kidney Tubulogenesis. J Biol Chem 283, 4272-4282.

Hotary, K., Allen, E., Punturieri, A., Yana, I., and Weiss, S.J. (2000). Regulation of Cell Invasion and Morphogenesis in a Three-Dimensional Type I Collagen Matrix by MembraneType Matrix Metalloproteinases 1, 2, and 3. J Cell Biol 149, 1309-1323.

Hyafil, F., Morello, D., Babinet, C., and Jacob, F. (1980). A cell surface glycoprotein involved in the compaction of embryonal carcinoma cells and cleavage stage embryos. Cell 21, 927-934.

Ishibe, S., Karihaloo, A., Ma, H., Zhang, J., Marlier, A., Mitobe, M., Togawa, A., Schmitt, R., Czyczk, J., Kashgarian, M., Geller, D.S., Thorgeirsson, S.S., and Cantley, L.G. (2009). Met and the epidermal growth factor receptor act cooperatively to regulate final nephron number and maintain collecting duct morphology. Development 136, 337-345.

Ito, A., Lai, C.-H., Zhao, X., Saito, S.i., Hamilton, M.H., Appella, E., and Yao, T.-P. (2001). p300/CBP-mediated p53 acetylation is commonly induced by p53-activating agents and inhibited by MDM2. EMBO J. 20, 1331-1340.

Jayaraman, G., Srinivas, R., Duggan, C., Ferreira, E., Swaminathan, S., Somasundaram, K., Williams, J., Hauser, C., Kurkinen, M., Dhar, R., Weitzman, S., Buttice, G., and Thimmapaya, B. (1999). p300/cAMP-responsive Element-binding Protein Interactions with Ets-1 and Ets-2 in 
the Transcriptional Activation of the Human Stromelysin Promoter. J Biol Chem 274, 1734217352.

Ji, A., Dao, D., Chen, J., and MacLellan, W.R. (2003). EID-2, a novel member of the EID family of p300-binding proteins inhibits transactivation by MyoD. Gene 318, 35-43.

Jin, Q., Yu, L.-R., Wang, L., Zhang, Z., Kasper, L.H., Lee, J.-E., Wang, C., Brindle, P.K., Dent, S.Y.R., and Ge, K. (2011). Distinct roles of GCN5/PCAF-mediated H3K9ac and CBP/p300mediated H3K18/27ac in nuclear receptor transactivation. EMBO J. 30, 249-262.

Jorda, M., Olmeda, D., Vinyals, A., Valero, E., Cubillo, E., Llorens, A., Cano, A., and Fabra, A. (2005). Upregulation of MMP-9 in MDCK epithelial cell line in response to expression of the Snail transcription factor. J Cell Sci 118, 3371-3385.

Jung, Y.S., Liu, X.-W., Chirco, R., Warner, R.B., Fridman, R., and Kim, H.-R.C. (2012). TIMP1 Induces an EMT-Like Phenotypic Conversion in MDCK Cells Independent of Its MMPInhibitory Domain. PLoS ONE 7, e38773.

Kamei, Y., Xu, L., Heinzel, T., Torchia, J., Kurokawa, R., Gloss, B., Lin, S.-C., Heyman, R.A., Rose, D.W., Glass, C.K., and Rosenfeld, M.G. (1996). A CBP Integrator Complex Mediates Transcriptional Activation and AP-1 Inhibition by Nuclear Receptors. Cell 85, 403-414.

Kasper, L.H., Lerach, S., Wang, J., Wu, S., Jeevan, T., and Brindle, P.K. (2010). CBP/p300 double null cells reveal effect of coactivator level and diversity on CREB transactivation. EMBO J. 29, 3660-3672.

Kim, D., Pertea, G., Trapnell, C., Pimentel, H., Kelley, R., and Salzberg, S.L. (2013). TopHat2: accurate alignment of transcriptomes in the presence of insertions, deletions and gene fusions. Genome Biol 14. 
Kohn, K.W., Zeeberg, B.M., Reinhold, W.C., and Pommier, Y. (2014). Gene expression correlations in human cancer cell lines define molecular interaction networks for epithelial phenotype. PLoS One 9, e99269.

Kokoszynska, K., Ostrowski, J., Rychlewski, L., and Wyrwicz, L.S. (2008). The fold recognition of CP2 transcription factors gives new insights into the function and evolution of tumor suppressor protein p53. Cell Cycle 7, 2907-2915.

Kumar, S., Park, S.H., Cieply, B., Schupp, J., Killiam, E., Zhang, F., Rimm, D.L., and Frisch, S.M. A pathway for the control of anoikis sensitivity by E-cadherin and epithelial-tomesenchymal transition. Mol Cell Biol 31, 4036-4051.

Kutluay, S.B., DeVos, S.L., Klomp, J.E., and Triezenberg, S.J. (2009). Transcriptional Coactivators Are Not Required for Herpes Simplex Virus Type 1 Immediate-Early Gene Expression In Vitro. J Virol. 83, 3436-3449.

Larue, L., Ohsugi, M., Hirchenhain, J., and Kemler, R. (1994). E-cadherin null mutant embryos fail to form a trophectoderm epithelium. Proc Natl Acad Sci USA. 91, 8263-8267.

Lee, M., and Partridge, N.C. (2010). Parathyroid Hormone Activation of Matrix Metalloproteinase-13 Transcription Requires the Histone Acetyltransferase Activity of p300 and PCAF and p300-dependent Acetylation of PCAF. J Biol Chem 285, 38014-38022.

Leroy, P., and Mostov, K.E. (2007). Slug Is Required for Cell Survival during Partial EpithelialMesenchymal Transition of HGF-induced Tubulogenesis. Mol Biol Cell 18, 1943-1952.

Li, J., Sutter, C., Parker, D.S., Blauwkamp, T., Fang, M., and Cadigan, K.M. (2007). CBP/p300 are bimodal regulators of Wnt signaling. EMBO J. 26, 2284-2294.

Li, L., and Li, W. (2015). Epithelial-mesenchymal transition in human cancer: Comprehensive reprogramming of metabolism, epigenetics, and differentiation. PharmacolTher. 150, 33-46. 
Lin, C.H., Hare, B.J., Wagner, G., Harrison, S.C., Maniatis, T., and Fraenkel, E. (2001). A Small Domain of CBP/p300 Binds Diverse Proteins: Solution Structure and Functional Studies. Mol Cell 8, 581-590.

Lu, B.C., Cebrian, C., Chi, X., Kuure, S., Kuo, R., Bates, C.M., Arber, S., Hassell, J., MacNeil, L., Hoshi, M., Jain, S., Asai, N., Takahashi, M., Schmidt-Ott, K.M., Barasch, J., D'Agati, V., and Costantini, F. (2009). Etv4 and Etv5 are required downstream of GDNF and Ret for kidney branching morphogenesis. Nat Genet 41, 1295-1302.

Ma, H., Guo, M., Shan, B., and Xia, Z. (2012). Targeted functional analysis of p300 coactivator in Wnt/ $\beta$-catenin signaling pathway using phosphoproteomic and biochemical approaches. J Proteomics 75, 2601-2610.

MacLellan, W.R., Xiao, G., Abdellatif, M., and Schneider, M.D. (2000). A Novel Rb- and p300Binding Protein Inhibits Transactivation by MyoD. Mol Cell Biol. 20, 8903-8915.

Matsuda, S., Harries, J.C., Viskaduraki, M., Troke, P.J.F., Kindle, K.B., Ryan, C., and Heery, D.M. (2004). A Conserved $\alpha$-Helical Motif Mediates the Binding of Diverse Nuclear Proteins to the SRC1 Interaction Domain of CBP. J. Biol Chem 279, 14055-14064.

Matthews, C.P., Colburn, N.H., and Young, M.R. (2007). AP-1 a target for cancer prevention. Curr Cancer Drug Targets 7, 317-324.

Miller, R.K., and McCrea, P.D. (2010). Wnt to Build a Tube: Contributions of Wnt signaling to epithelial tubulogenesis. Dev Dyn. 239, 77-93.

Miyake, S., Yanagisawa, Y., and Yuasa, Y. (2003). A Novel EID-1 Family Member, EID-2, Associates with Histone Deacetylases and Inhibits Muscle Differentiation. J. Biol Chem. 278, 17060-17065. 
Mizzen, A.C., and Allis, D.C. Linking histone acetylation to transcriptional regulation. Cell Mol Life Sci. 54, 6-20.

Mlacki, M., Kikulska, A., Krzywinska, E., Pawlak, M., and Wilanowski, T. (2015). Recent discoveries concerning the involvement of transcription factors from the Grainyhead-like family in cancer. Exp Biol Med (Maywood) 240, 1396-1401.

Monga, S.P.S., Mars, W.M., Pediaditakis, P., Bell, A., Mulé, K., Bowen, W.C., Wang, X., Zarnegar, R., and Michalopoulos, G.K. (2002). Hepatocyte Growth Factor Induces Wntindependent Nuclear Translocation of $\beta$-Catenin after Met- $\beta$-Catenin Dissociation in Hepatocytes. Cancer Res 62, 2064-2071.

Narayanan, K., Srinivas, R., Peterson, M.C., Ramachandran, A., Hao, J., Thimmapaya, B., Scherer, P.E., and George, A. (2004). Transcriptional Regulation of Dentin Matrix Protein 1 by JunB and p300 during Osteoblast Differentiation. J Biol Chem. 279, 44294-44302.

Nonaka, T., Nishibashi, K., Itoh, Y., Yana, I., and Seiki, M. (2005). Competitive disruption of the tumor-promoting function of membrane type 1 matrix metalloproteinase/matrix metalloproteinase-14 in vivo. Mol Cancer Ther 4, 1157-1166.

O'Brien, L.E., Zegers, M.M.P., and Mostov, K.E. (2002). Building epithelial architecture: insights from three-dimensional culture models. Nat Rev Mol Cell Biol 3, 531-537.

Ogryzko, V.V., Schiltz, R.L., Russanova, V., Howard, B.H., and Nakatani, Y. (1996). The Transcriptional Coactivators p300 and CBP Are Histone Acetyltransferases. Cell 87, 953-959. Oh, S., Shin, S., and Janknecht, R. (2012). ETV1, 4 and 5: An Oncogenic Subfamily of ETS Transcription Factors. Biochim Biophys Acta. 1826, 1-12.

Oike, Y., Takakura, N., Hata, A., Kaname, T., Akizuki, M., Yamaguchi, Y., Yasue, H., Araki, K., Yamamura, K.-i., and Suda, T. (1999). Mice Homozygous for a Truncated Form of CREB- 
Binding Protein Exhibit Defects in Hematopoiesis and Vasculo-angiogenesis. Blood 93, 27712779.

Polesskaya, A., Naguibneva, I., Fritsch, L., Duquet, A., Ait-Si-Ali, S., Robin, P., Vervisch, A., Pritchard, L.L., Cole, P., and Harel-Bellan, A. (2001). CBP/p300 and muscle differentiation: no HAT, no muscle. EMBO J. 20, 6816-6825.

Pollack, A.L., Apodaca, G., and Mostov, K.E. (2004). Hepatocyte growth factor induces MDCK cell morphogenesis without causing loss of tight junction functional integrity. Am J Physiol Cell Physiol 286, C482-C494.

Pollack, A.L., Runyan, R.B., and Mostov, K.E. (1998). Morphogenetic Mechanisms of Epithelial Tubulogenesis: MDCK Cell Polarity Is Transiently Rearranged without Loss of Cell-Cell Contact during Scatter Factor/Hepatocyte Growth Factor-Induced Tubulogenesis. Dev Biol/ 204, 64-79.

Polyak, K., and Weinberg, R.A. (2009). Transitions between epithelial and mesenchymal states: acquisition of malignant and stem cell traits. Nat Rev Cancer 9, 265-273.

Puri, P.L., Sartorelli, V., Yang, X.-J., Hamamori, Y., Ogryzko, V.V., Howard, B.H., Kedes, L., Wang, J.Y.J., Graessmann, A., Nakatani, Y., and Levrero, M. (1997). Differential Roles of p300 and PCAF Acetyltransferases in Muscle Differentiation. Mol Cell 1, 35-45.

Qin, L., Liu, Z., Chen, H., and Xu, J. (2009). The steroid receptor coactivator-1 regulates twist expression and promotes breast cancer metastasis. Cancer Res 69, 3819-3827.

Rifat, Y., Parekh, V., Wilanowski, T., Hislop, N.R., Auden, A., Ting, S.B., Cunningham, J.M., and Jane, S.M. Regional neural tube closure defined by the Grainy head-like transcription factors. Dev Biol 345, 237-245. 
Ringel, A.E., and Wolberger, C. (2013). A new RING tossed into an old HAT. Structure 21, 1479-1481.

Santer, F.R., Hoschele, P.P., Oh, S.J., Erb, H.H., Bouchal, J., Cavarretta, I.T., Parson, W., Meyers, D.J., Cole, P.A., and Culig, Z. (2011). Inhibition of the acetyltransferases p300 and CBP reveals a targetable function for p300 in the survival and invasion pathways of prostate cancer cell lines. Mol Cancer Ther 10, 1644-1655.

Schmidt-Ott, K.M., Yang, J., Chen, X., Wang, H., Paragas, N., Mori, K., Li, J.-Y., Lu, B., Costantini, F., Schiffer, M., Bottinger, E., and Barasch, J. (2005). Novel Regulators of Kidney Development from the Tips of the Ureteric Bud. J Am Soc Nephrol 16, 1993-2002.

Senga, K., Mostov, K.E., Mitaka, T., Miyajima, A., and Tanimizu, N. Grainyhead-like 2 regulates epithelial morphogenesis by establishing functional tight junctions through the organization of a molecular network among claudin3, claudin4, and Rab25. Mol Biol Cell 23, $2845-2855$.

Sheppard, H.M., Harries, J.C., Hussain, S., Bevan, C., and Heery, D.M. (2001). Analysis of the Steroid Receptor Coactivator 1 (SRC1)-CREB Binding Protein Interaction Interface and Its Importance for the Function of SRC1. Mol Cell Biol 21, 39-50.

Snowden, A.W., Anderson, L.A., Webster, G.A., and Perkins, N.D. (2000). A Novel Transcriptional Repression Domain Mediates p21WAF1/CIP1 Induction of p300 Transactivation. Mol Cell Biol 20, 2676-2686.

Stiehl, D.P., Fath, D.M., Liang, D., Jiang, Y., and Sang, N. (2007). Histone Deacetylase Inhibitors Synergize p300 Autoacetylation that Regulates Its Transactivation Activity and Complex Formation. Cancer Res.67, 2256-2264. 
Sun, Y., Zeng, X.-R., Wenger, L., Firestein, G.S., and Cheung, H.S. (2004). p53 down-regulates matrix metalloproteinase- 1 by targeting the communications between AP-1 and the basal transcription complex. J Cell Biochem 92, 258-269.

Tam, W.L., and Weinberg, R.A. (2013). The epigenetics of epithelial-mesenchymal plasticity in cancer. Nat Med 19, 1438-1449.

Tang, W., and Hemler, M.E. (2004). Caveolin-1 Regulates Matrix Metalloproteinases-1 Induction and CD147/EMMPRIN Cell Surface Clustering. J. Biol Chem. 279, 11112-11118.

Tang, Z., Chen, W.-Y., Shimada, M., Nguyen, Uyen T.T., Kim, J., Sun, X.-J., Sengoku, T., McGinty, Robert K., Fernandez, Joseph P., Muir, Tom W., and Roeder, Robert G. (2013). SET1 and p300 Act Synergistically, through Coupled Histone Modifications, in Transcriptional Activation by p53. Cell 154, 297-310.

Tanimizu, N., and Mitaka, T. (2013). Role of grainyhead-like 2 in the formation of functional tight junctions. Tissue Barriers 1, e23495.

Teo, J.-L., and Kahn, M. (2010). The Wnt signaling pathway in cellular proliferation and differentiation: A tale of two coactivators. Advanced Drug Delivery Reviews 62, 1149-1155.

Thiery, J.P., and Sleeman, J.P. (2006). Complex networks orchestrate epithelial-mesenchymal transitions. Nat Rev Mol Cell Biol 7, 131-142.

Thompson, P.R., Wang, D., Wang, L., Fulco, M., Pediconi, N., Zhang, D., An, W., Ge, Q., Roeder, R.G., Wong, J., Levrero, M., Sartorelli, V., Cotter, R.J., and Cole, P.A. (2004). Regulation of the p300 HAT domain via a novel activation loop. Nat Struct Mol Biol 11, 308315. 
Ting, S.B., Caddy, J., Hislop, N., Wilanowski, T., Auden, A., Zhao, L.L., Ellis, S., Kaur, P., Uchida, Y., Holleran, W.M., Elias, P.M., Cunningham, J.M., and Jane, S.M. (2005). A homolog of Drosophila grainy head is essential for epidermal integrity in mice. Science 308, 411-413.

Ting, S.B., Wilanowski, T., Cerruti, L., Zhao, L.L., Cunningham, J.M., and Jane, S.M. (2003). The identification and characterization of human Sister-of-Mammalian Grainyhead (SOM) expands the grainyhead-like family of developmental transcription factors. Biochem J 370, 953962.

Verschure, P.J., van der Kraan, I., de Leeuw, W., van der Vlag, J., Carpenter, A.E., Belmont, A.S., and van Driel, R. (2005). In Vivo HP1 Targeting Causes Large-Scale Chromatin Condensation and Enhanced Histone Lysine Methylation. Mol Cell Biol 25, 4552-4564. Vestweber, D., and Kemler, R. (1985). Identification of a putative cell adhesion domain of uvomorulin. EMBO J. 4, 3393-3398.

Vo, N., and Goodman, R.H. (2001). CREB-binding Protein and p300 in Transcriptional Regulation. J Biol Chem 276, 13505-13508.

Walentin, K., Hinze, C., Werth, M., Haase, N., Varma, S., Morell, R., Aue, A., Pötschke, E., Warburton, D., Qiu, A., Barasch, J., Purfürst, B., Dieterich, C., Popova, E., Bader, M., Dechend, R., Staff, A.C., Yurtdas, Z.Y., Kilic, E., and Schmidt-Ott, K.M. (2015). A Grhl2-dependent gene network controls trophoblast branching morphogenesis. Development 142, 1125-1136.

Wang, A.Z., Ojakian, G.K., and Nelson, W.J. (1990). Steps in the morphogenesis of a polarized epithelium. I. Uncoupling the roles of cell-cell and cell-substratum contact in establishing plasma membrane polarity in multicellular epithelial (MDCK) cysts. J Cell Sci 95, 137-151.

Wang, S., and Samakovlis, C. (2012). Grainy head and its target genes in epithelial morphogenesis and wound healing. Curr Top Dev Biol 98, 35-63. 
Watanabe, K., Villarreal-Ponce, A., Sun, P., Salmans, Michael L., Fallahi, M., Andersen, B., and Dai, X. (2014). Mammary Morphogenesis and Regeneration Require the Inhibition of EMT at Terminal End Buds by Ovol2 Transcriptional Repressor. Dev Cell 29, 59-74.

Werth, M., Walentin, K., Aue, A., Schönheit, J., Wuebken, A., Pode-Shakked, N., Vilianovitch, L., Erdmann, B., Dekel, B., Bader, M., Barasch, J., Rosenbauer, F., Luft, F.C., and Schmidt-Ott, K.M. (2010). The transcription factor grainyhead-like 2 regulates the molecular composition of the epithelial apical junctional complex. Development 137, 3835-3845.

Westermarck, J., and KÄHÄRi, V.-M. (1999). Regulation of matrix metalloproteinase expression in tumor invasion. The FASEB Journal 13, 781-792.

Wilanowski, T., Tuckfield, A., Cerruti, L., O'Connell, S., Saint, R., Parekh, V., Tao, J., Cunningham, J.M., and Jane, S.M. (2002). A highly conserved novel family of mammalian developmental transcription factors related to Drosophila grainyhead. Mech Dev 114, 37-50. Witte, S., Bradley, A., Enright, A.J., and Muljo, S.A. (2015). High-density P300 enhancers control cell state transitions. BMC Genomics 16, 1-13.

Wolf, D., Rodova, M., Miska, E.A., Calvet, J.P., and Kouzarides, T. (2002). Acetylation of $\beta$ Catenin by CREB-binding Protein (CBP). J Bioll Chem 277, 25562-25567.

Wollenick, K., Hu, J., Kristiansen, G., Schraml, P., Rehrauer, H., Berchner-Pfannschmidt, U., Fandrey, J., Wenger, R.H., and Stiehl, D.P. (2012). Synthetic transactivation screening reveals ETV4 as broad coactivator of hypoxia-inducible factor signaling. Nucleic Acids Res 40, 19281943.

Xu, Y., Hu, B., Qin, L., Zhao, L., Wang, Q., Wang, Q., Xu, Y., and Jiang, J. (2014). SRC-1 and Twist1 expression positively correlates with a poor prognosis in human breast cancer. Int J Biol Sci 10, 396-403. 
Yang, C., Shapiro, L.H., Rivera, M., Kumar, A., and Brindle, P.K. (1998). A Role for CREB Binding Protein and p300 Transcriptional Coactivators in Ets-1 Transactivation Functions. Mol Cell Biol 18, 2218-2229.

Yang, H., Pinello, C.E., Luo, J., Li, D., Wang, Y., Zhao, L.Y., Jahn, S.C., Saldanha, S.A., Chase, P., Planck, J., Geary, K.R., Ma, H., Law, B.K., Roush, W.R., Hodder, P., and Liao, D. (2013). Small-molecule inhibitors of acetyltransferase p300 identified by high-throughput screening are potent anticancer agents. Mol Cancer Ther 12, 610-620.

Yao, T.-P., Oh, S.P., Fuchs, M., Zhou, N.-D., Ch'ng, L.-E., Newsome, D., Bronson, R.T., Li, E., Livingston, D.M., and Eckner, R. Gene Dosage (2013);Dependent Embryonic Development and Proliferation Defects in Mice Lacking the Transcriptional Integrator p300. Cell 93, 361-372.

Zhang, L., He, X., Liu, L., Jiang, M., Zhao, C., Wang, H., He, D., Zheng, T., Zhou, X., Hassan, A., Ma, Z., Xin, M., Sun, Z., Lazar, Mitchell A., Goldman, Steven A., Olson, Eric N., and Lu, Q.R. (2016). Hdac3 Interaction with p300 Histone Acetyltransferase Regulates the Oligodendrocyte and Astrocyte Lineage Fate Switch. Dev Cell 36, 316-330.

Zhang, Y., Yan, W., and Chen, X. (2014). P63 regulates tubular formation via epithelial-tomesenchymal transition. Oncogene 33, 1548-1557.

Zhao, H., Kegg, H., Grady, S., Truong, H.-T., Robinson, M.L., Baum, M., and Bates, C.M. (2004). Role of fibroblast growth factor receptors 1 and 2 in the ureteric bud. Dev Biol 276, 403415.

Zhou, B., Liu, Y., Kahn, M., Ann, D.K., Han, A., Wang, H., Nguyen, C., Flodby, P., Zhong, Q., Krishnaveni, M.S., Liebler, J.M., Minoo, P., Crandall, E.D., and Borok, Z. (2012). Interactions between beta-catenin and transforming growth factor-beta signaling pathways mediate epithelialmesenchymal transition and are dependent on the transcriptional co-activator cAMP-response element-binding protein (CREB)-binding protein (CBP). J Biol Chem 287, 7026-7038. 
Figure Legends

Figure 1. GRHL2 suppresses HGF-induced cell scattering and tubulogenesis, and is downregulated by HGF. A. HGF induction downregulates endogenous GRHL2 protein. Western blot and densitometric quantitation of HGF treatment time course in MDCK cells are shown. HGF induction downregulates endogenous GRHL2 mRNA in MDCK cells; qPCR results expressed as ratios compared to vector control. B. Constitutive GRHL2 expression in MDCK cells suppresses HGF-induced cell scattering; images represent representative morphologies of cells treated for 42 hours. C. GRHL2 knockdown in enhanced HGF-induced cell scattering (16 hours), but MDCK shGRHL2 cells did not undergo an EMT phenotypic change when EMT markers were examined via western blotting. D. Constitutive GRHL2 expression in MDCK cells prevents tubulogenesis (blue-nuclei and green-actin). Scale bar equals 20 microns. Graph represents quantification of percentage of cysts that demonstrated tubulogenesis.

Figure 2. GRHL2 suppresses the induction of MMP genes and MMP promoters by HGF. A. GRHL2 suppresses the induction of MMP genes (qPCR analysis; EN: endogenous GRHL2, CE: constitutively expressed GRHL2, KD: GRHL2 shRNA knockdown). B. GRHL2 suppresses the induction of MMP promoters by HGF. HT1080 cells were cotransfected with either MMP1 or MMP14 promoter-luciferase reporter constructs and GRHL2, E1A, or empty expression vectors. Values represent relative luciferase activity normalized to TK- $\beta$-galactosidase control.

Figure 3. GRHL2 suppresses AP-1 and p300 function. A. GRHL2 suppresses AP-1 function. HT1080 and 293T cells were cotransfected with AP-1 response element- luciferase reporter construct and GRHL2 expression vector or empty vector. Phorbol 12-myristate 13-acetate (PMA) was used to induce AP-1 signaling in 293T cells. Values represent relative luciferase activity normalized to TK- $\beta$-galactosidase control. B. Venn diagram comparing GRHL2- 
repressed genes (Farris et al., 2016) vs. p300 target genes identified via E1a (Ferrari et al., 2014). C. GRHL2 suppresses the p300 pathway. p300 effector and target genes induced by HGF in MDCK cells with GRHL2 shRNA vs. cells with constitutive GRHL2 determined by IPA analysis quantitation of the number of unregulated, HGF up-regulated or HGF down-regulated genes for both cell lines is shown in the graph. The p300 interactome diagram on which this is based is shown in figure S7. D. GRHL2 suppresses p300 function. GAL4-minimal promoterluciferase activation by a co-transfected GAL4-p300 was assayed in HT1080 cells in the presence or absence of cotransfected GRHL2 expression vector; values represent relative luciferase activity normalized to TK- $\beta$-galactosidase control.

Figure 4. GRHL2 suppresses the HAT activity of p300. A. In vitro HAT assays with recombinant H3, p300 and GRHL2 proteins. Coomassie stains to assess the quality of recombinant proteins used throughout this study are shown in figure S19. B. GRHL2 inhibits the acetylation of H3 on GRHL2-repressed but not GRHL2-induced promoters in vivo (ChIP assay). Crosslinked chromatin from HT1080+vector HT-1080+GRHL2 cells immunoprecipitated with H3K27-Ac or rabbit IgG; the indicated promoters were assayed for H3K27Ac by qPCR using the primers in Materials and Methods. * indicates p values of $<0.05$. GRHL2 does not affect global histone H3K18-Ac or H3K27-Ac (western blotting of total histones). C. GRHL2 interacts with p300. (left panel): co-transfection of indicated expression constructs, followed by coimmunoprecipation/western blotting; (middle panel): co-immunoprecipitation of retrovirally expressed S-tagged GRHL2 with endogenous p300; (right panel): co-immunoprecipitation of endogenous GRHL2 and endogenous P300. 
Figure 5. GRHL2 inhibits the C-terminal transactivation domain of p300. A. The p300 HAT domain stimulates transactivation by the p300 C-terminus (co-transfection in HT1080 cells). B. GRHL2 inhibits transactivation by the p300 C-terminus. HT1080 cells were cotransfected with GAL4 response element luciferase reporter in the presence of the indicated expression constructs. C. The IBID domain of p300 and the co-activator SRC-1 synergize to activate transcription. HT1080 cells were cotransfected with GAL4 response element luciferase reporter I in the presence of the indicated expression constructs. D. GRHL2 inhibits transactivation by the IBID-SRC-1 complex. HT1080 cells were cotransfected with GAL4 response element luciferase reporter in the presence of the indicated expression constructs. E. Transactivation by the IBIDSRC-1 complex is contingent upon lysines 2086 and 2091. HT1080 cells were cotransfected with GAL4 response element luciferase reporter in the presence of the indicated expression constructs. (a-e):Values represent relative luciferase activity normalized to TK- $\beta$-galactosidase control. F. Schematic of p300 domains. KIX- CREB-binding domain, Bd- bromodomain, HAT histone acetyltransferase domain, and IBID- IRF-3 binding domain.

Figure 6. A small region within the DNA binding domain of GRHL2, amino acids 425-437, inhibits p300. A. Schematic of GRHL2 domains. TAD- transactivation domain , DBD- DNA binding domain, and DD- dimerization domain. B. HAT assays using the indicated GRHL2 fragments, assayed as GST-fusion proteins. C. GRHL2 aa425-437 inhibits p300 HAT activity. The indicated fragments derived from GRHL2 were assayed as GST-fusions (left panels) or as recombinant peptides (right panel) for inhibition of HAT activity. D. GRHL2 amino acids 425437 are required for inhibition of an AP-1 reporter, GAL4 reporter in conjunction with GAL4p300, MMP1 reporter or MMP14 reporter. Values represent relative luciferase activity normalized to TK- $\beta$-galactosidase control. 
Figure 7. The p300-inhibitory domain of GRHL2 is important for the suppression of tubulogenesis and reversion of EMT. A MDCK cells expressing GRHL2 wild-type or GRHL2 $\Delta 425$-437 were assayed for tubulogenesis (blue-nuclei and green-actin). Scale bar equals 20 microns.; quantitation is shown in the middle panel and Western blot confirmation of protein levels in the right panel. B. MSP cells expressing GRHL2 wild-type or GRHL2 $\Delta 425$-437 were assayed for reversion of EMT, by western blotting for EMT markers (left panel), cell morphology (middle panel), or qPCR (right panel).

Figure 8. GRHL2 suppresses tubulogenesis and EMT by inhibition of p300. 
A.
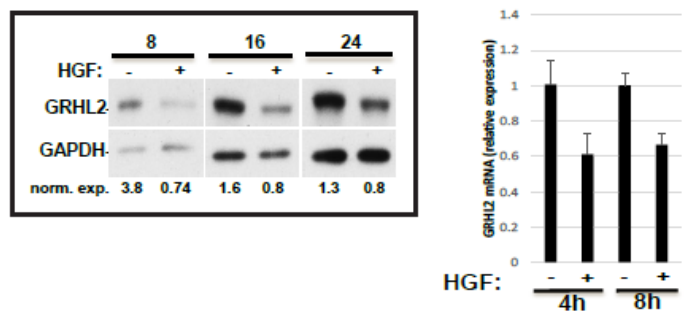

C.

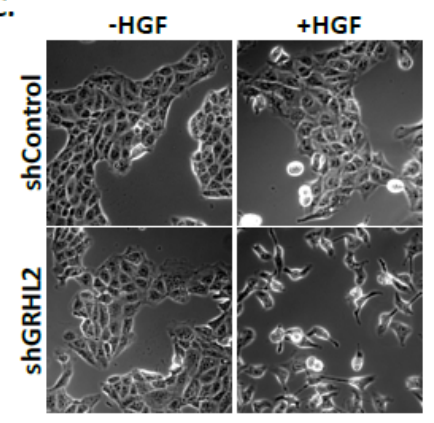

B.

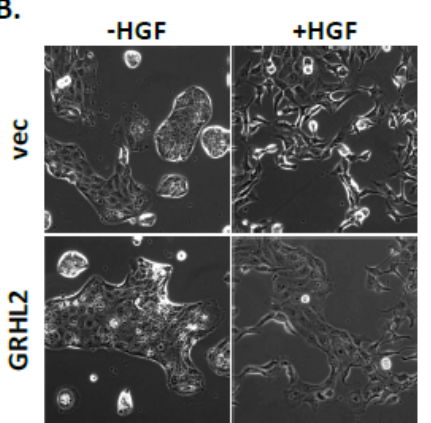

D.

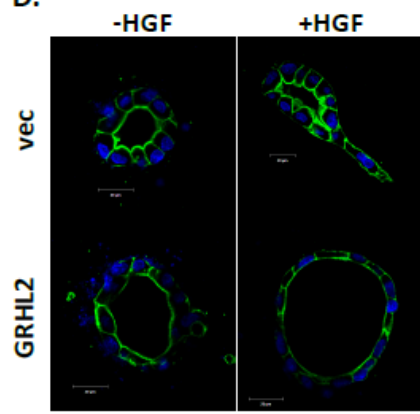

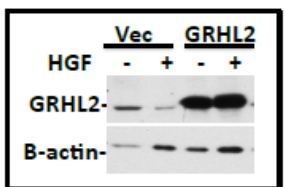

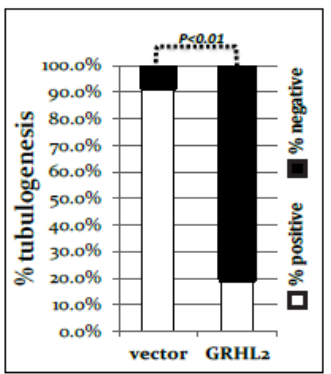

Figure 1 
A.

MMP1

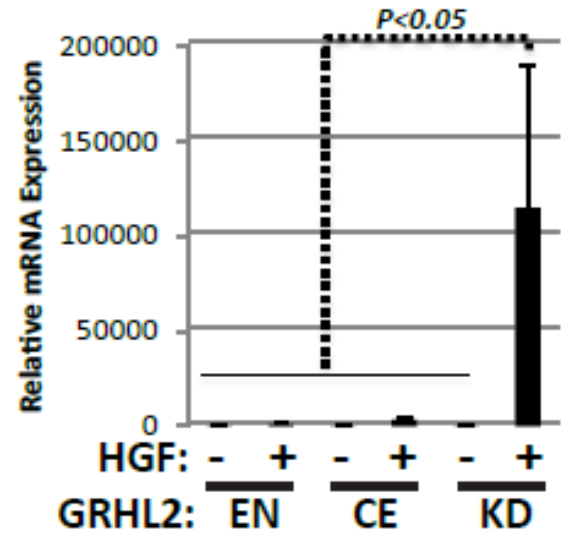

MMP-13

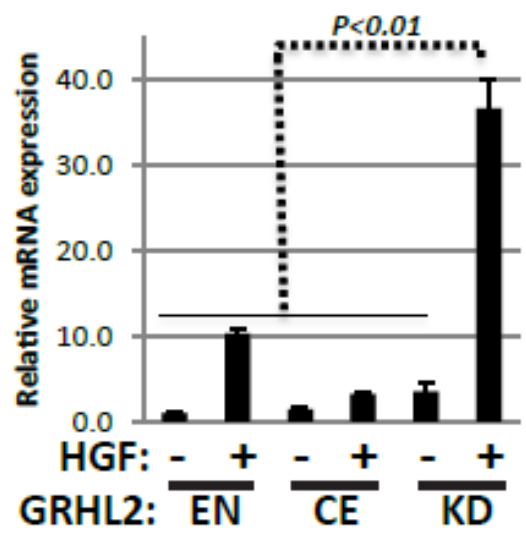

MMP-3

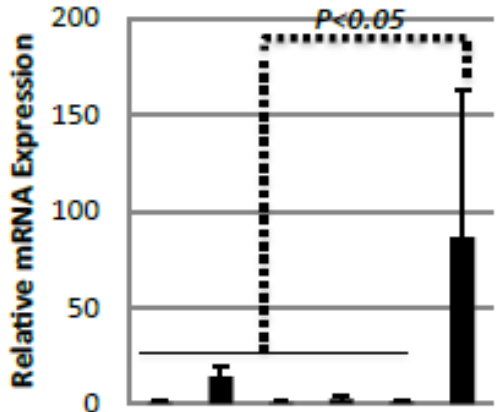

HGF: $\frac{-+}{\mathrm{RH}} \frac{-+}{\mathrm{CE}} \frac{-+}{\mathrm{KD}}$

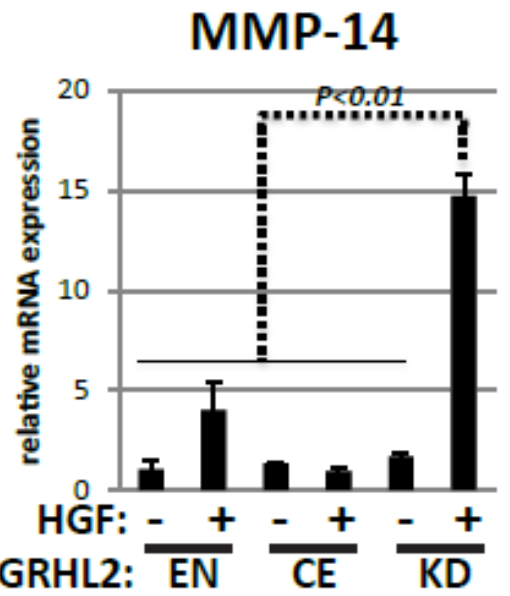

B.

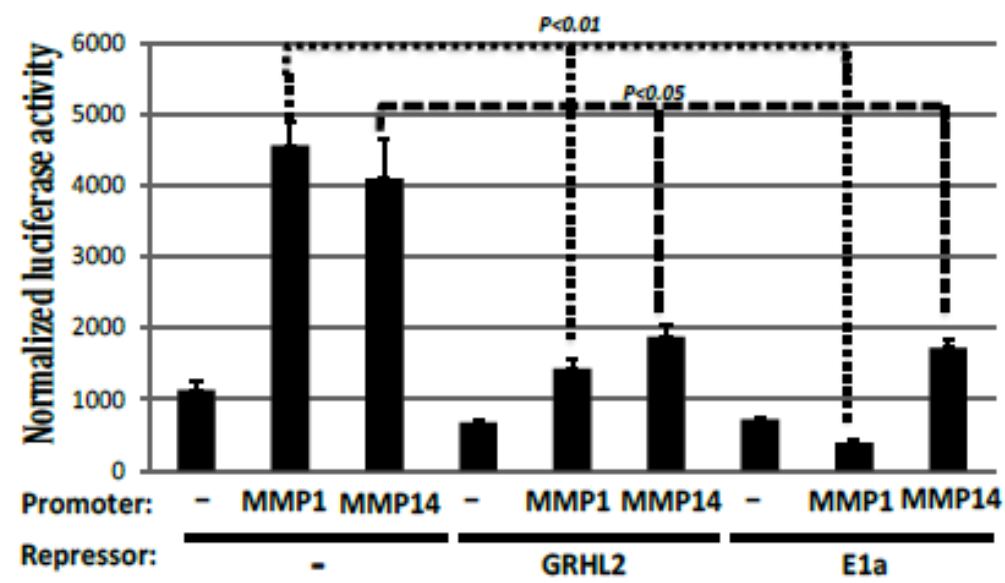

Figure 2 
A.
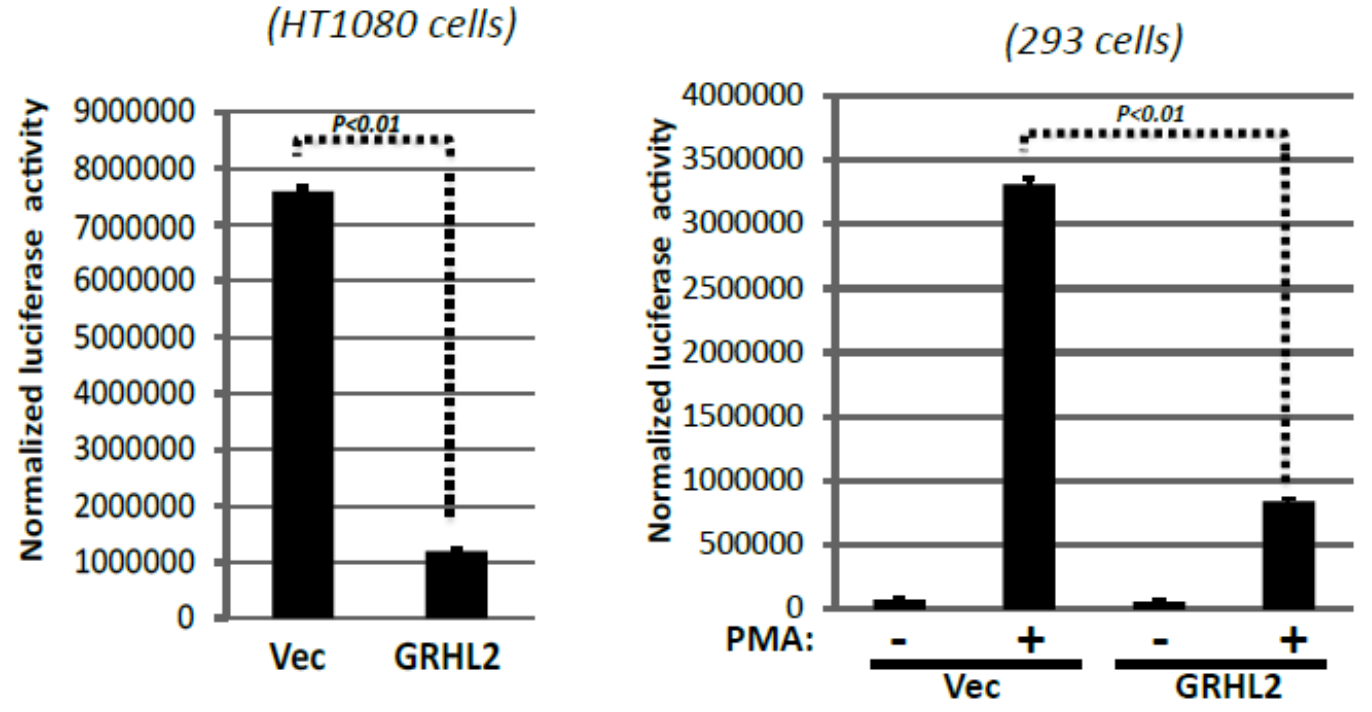

B.

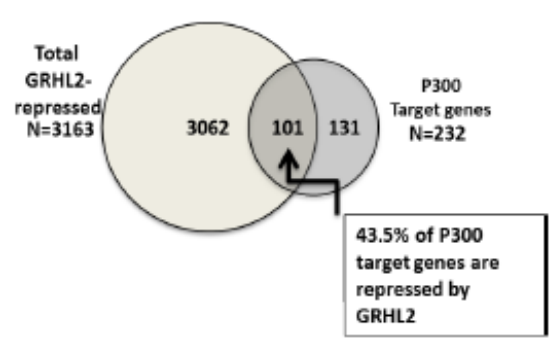

C.

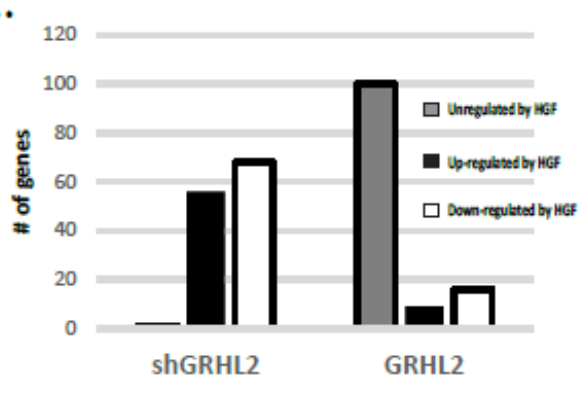

D.

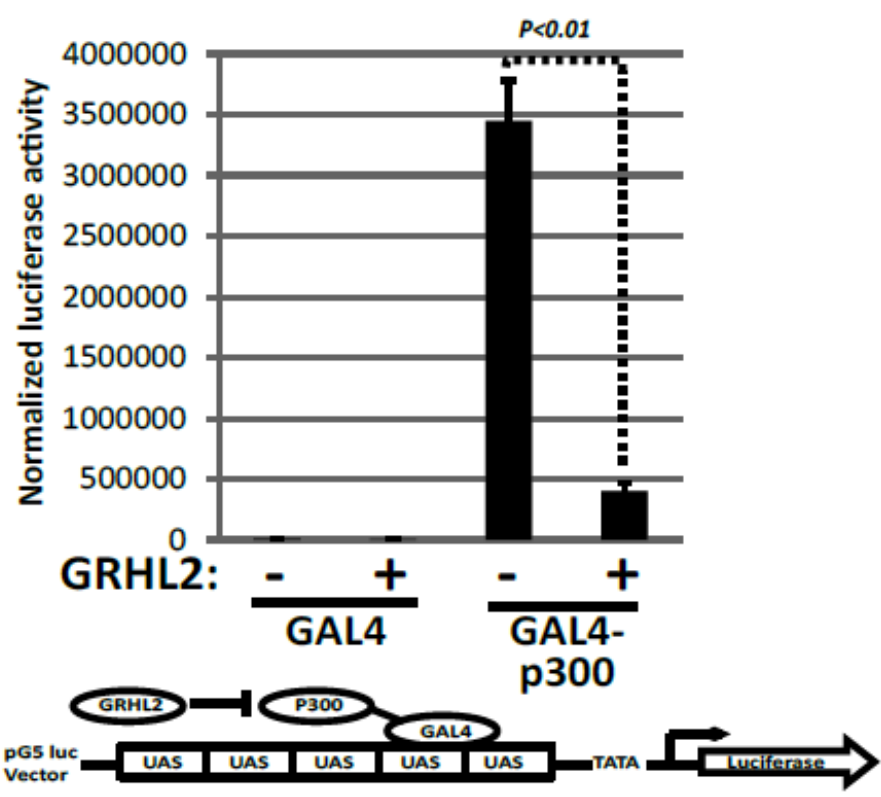

Figure 3 
A.

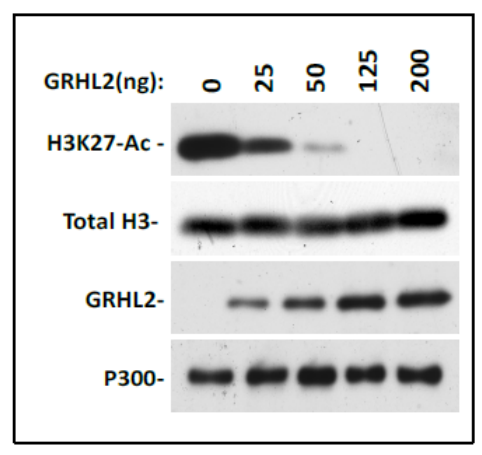

B.

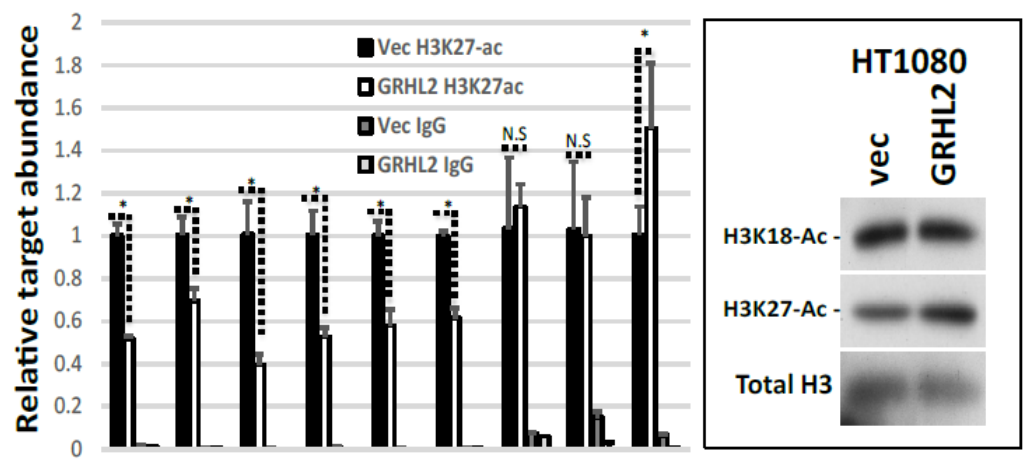

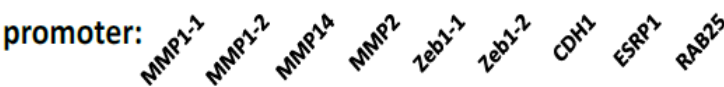

C.

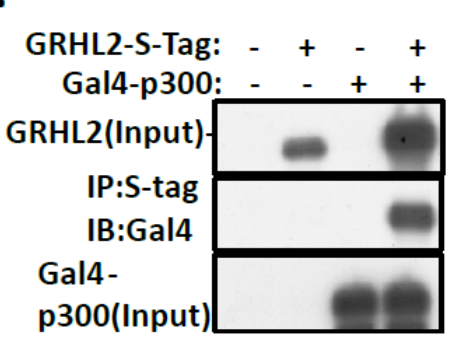

293 co-transfection

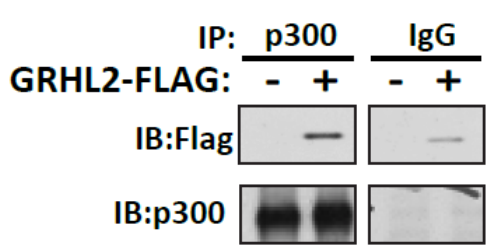

Endogenous p300 Ectopic GRHL2

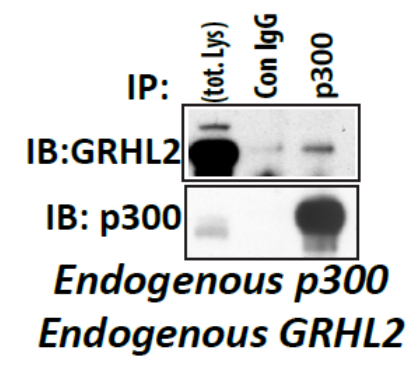

Figure 4 

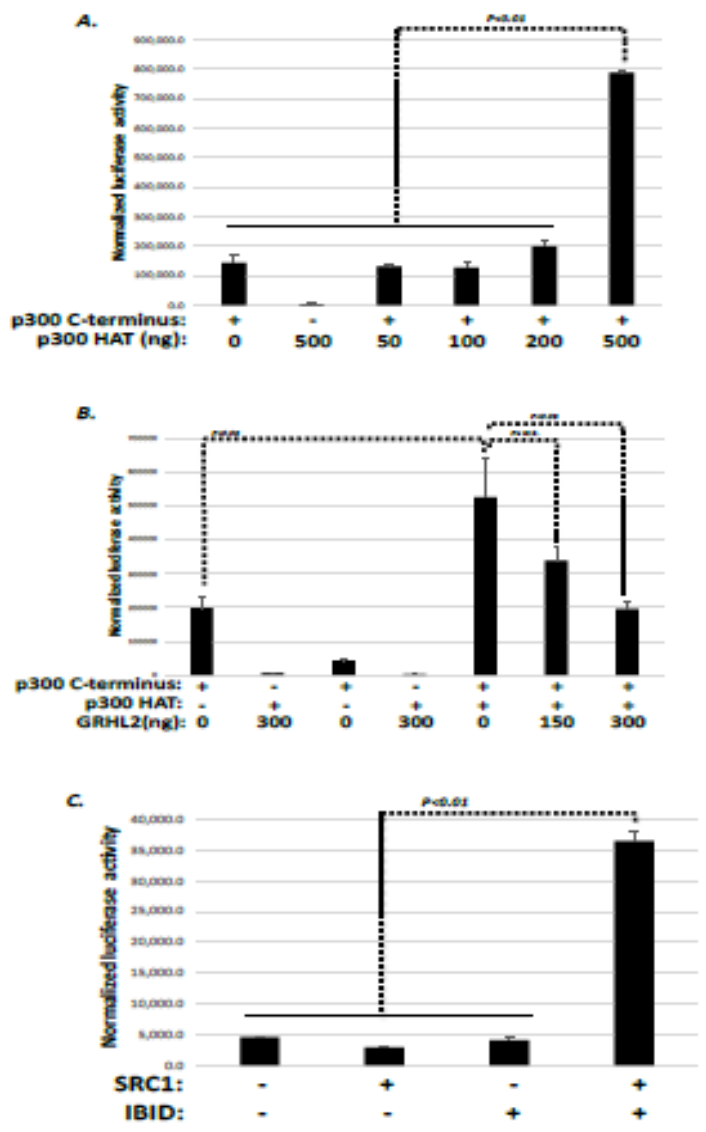

D.

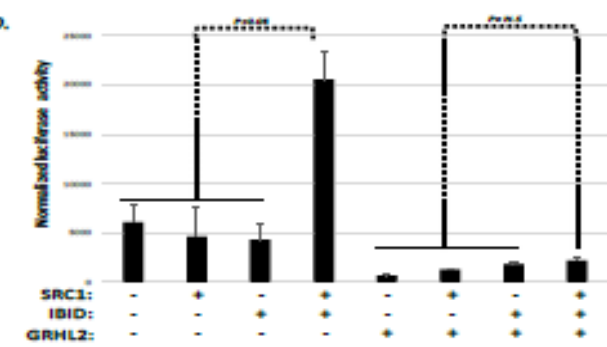

E.
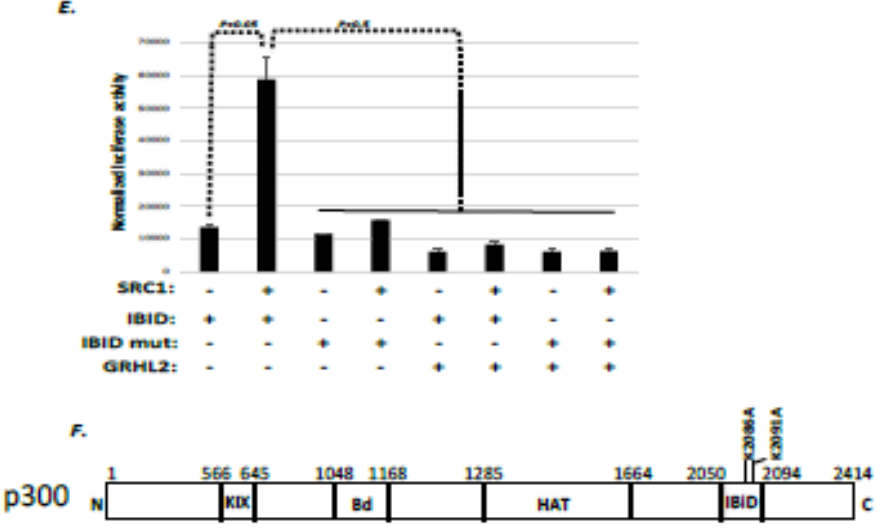

Figure 5 
A.
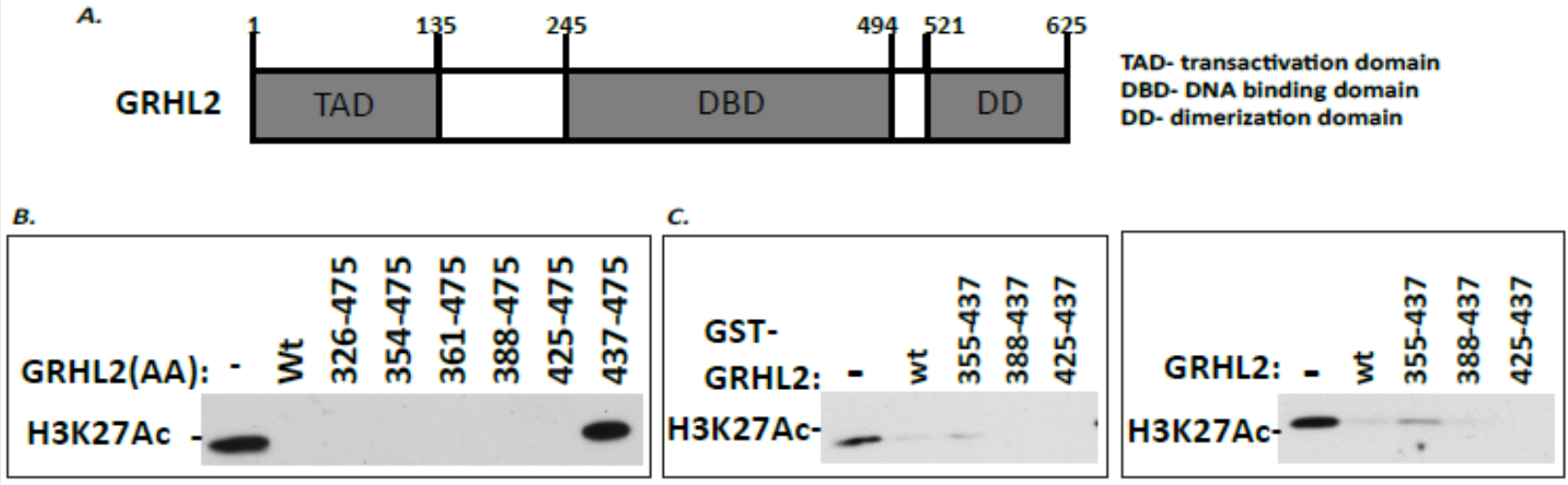

D. AP1 Responsive Element

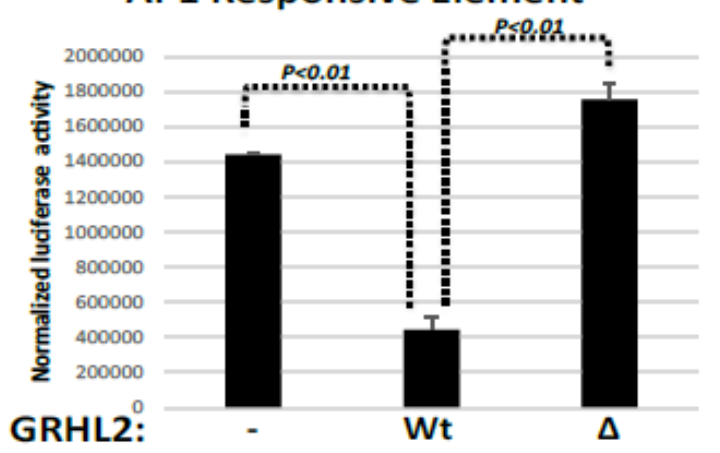

MMP1 promoter

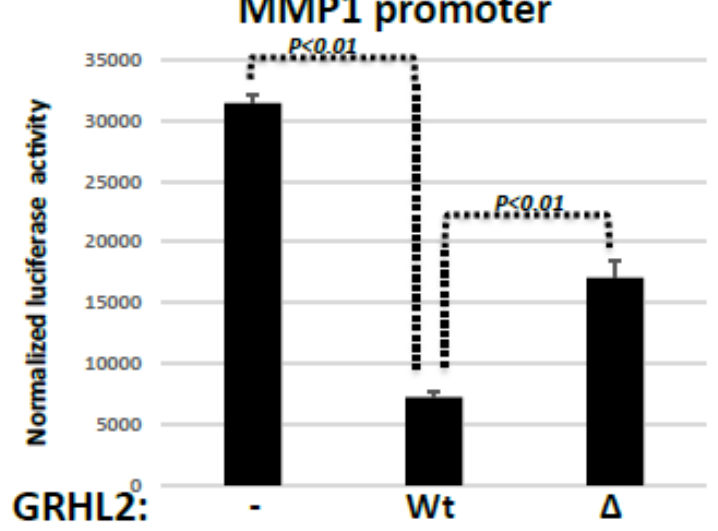

P300-GAL4 pG5

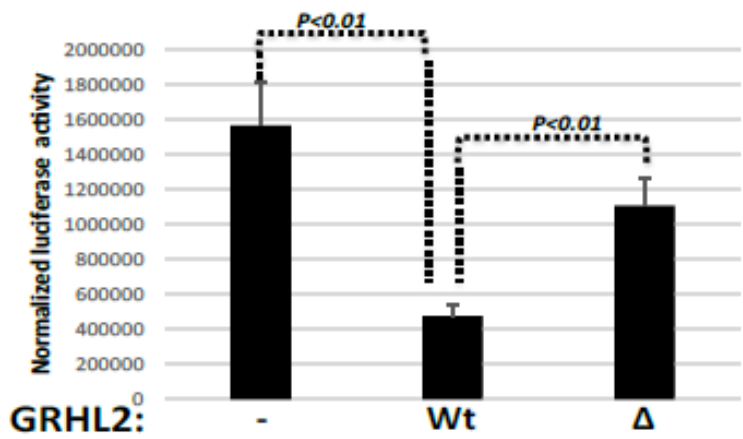

MMP14 promoter

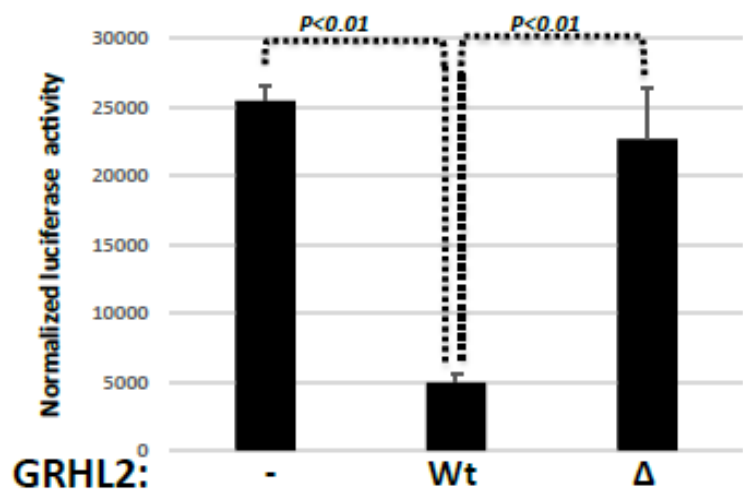

Figure 6 

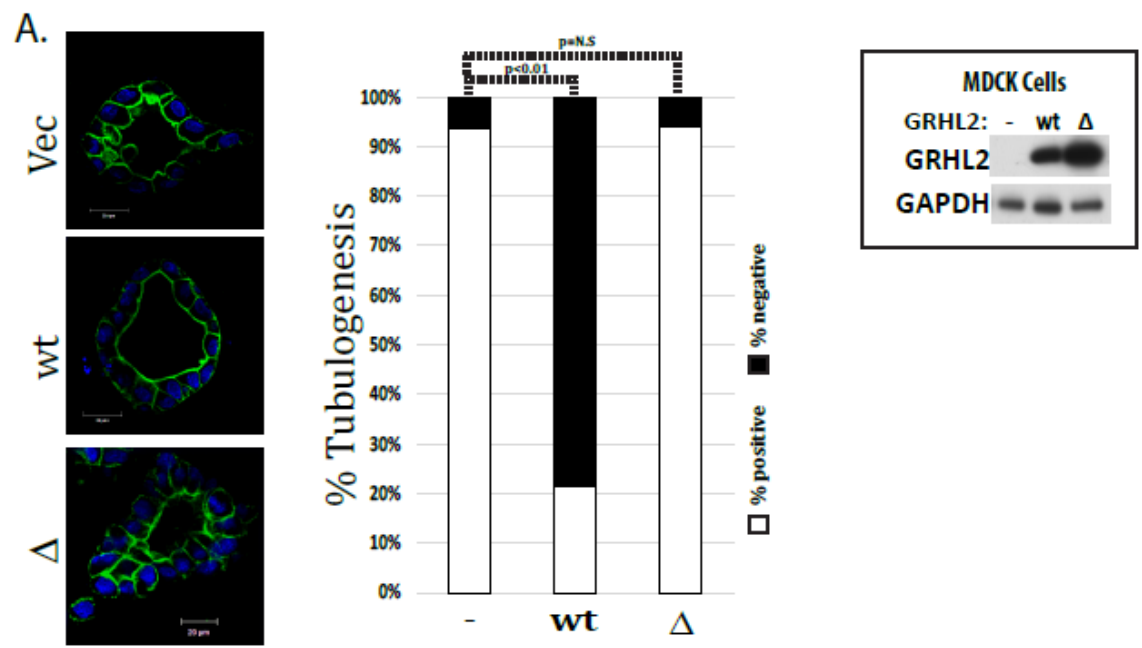

B.
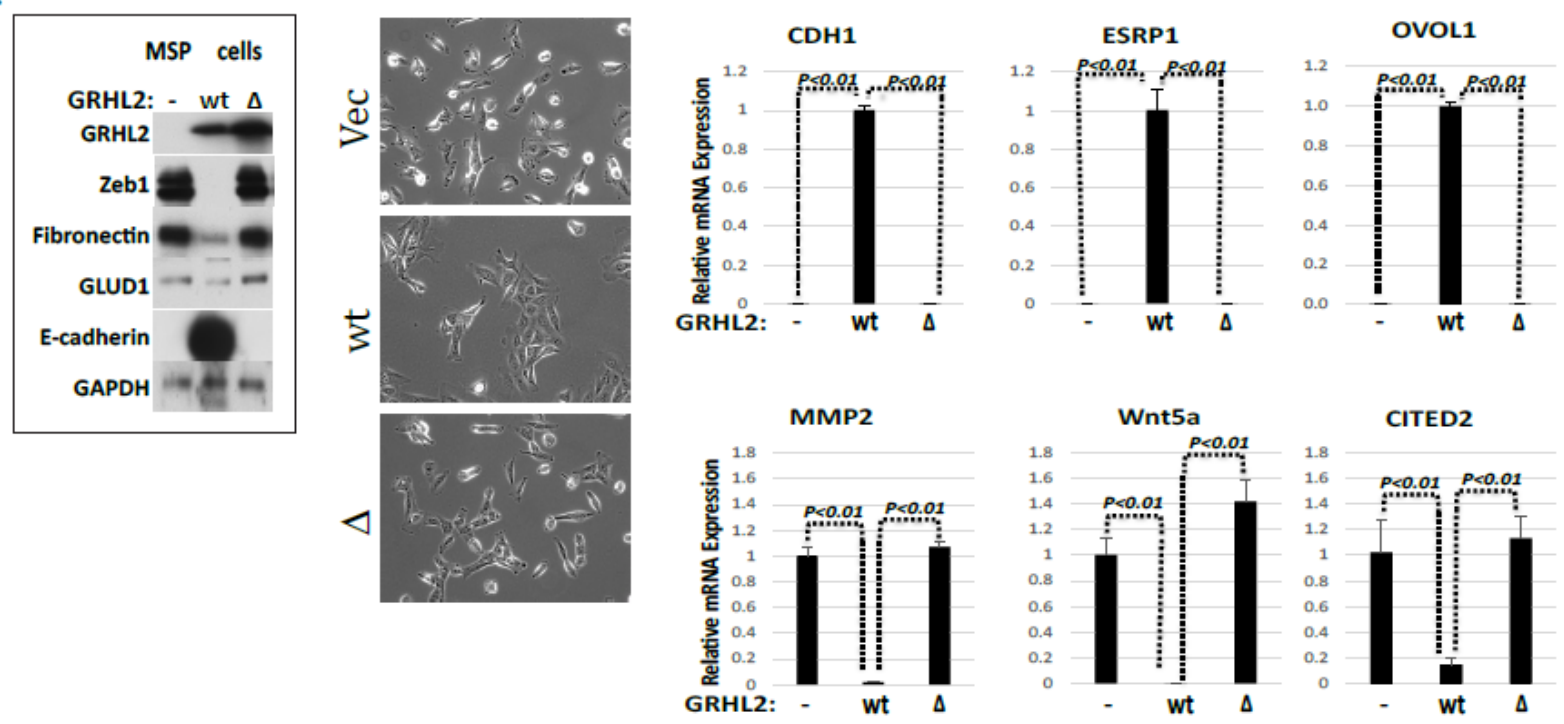

Figure 7 


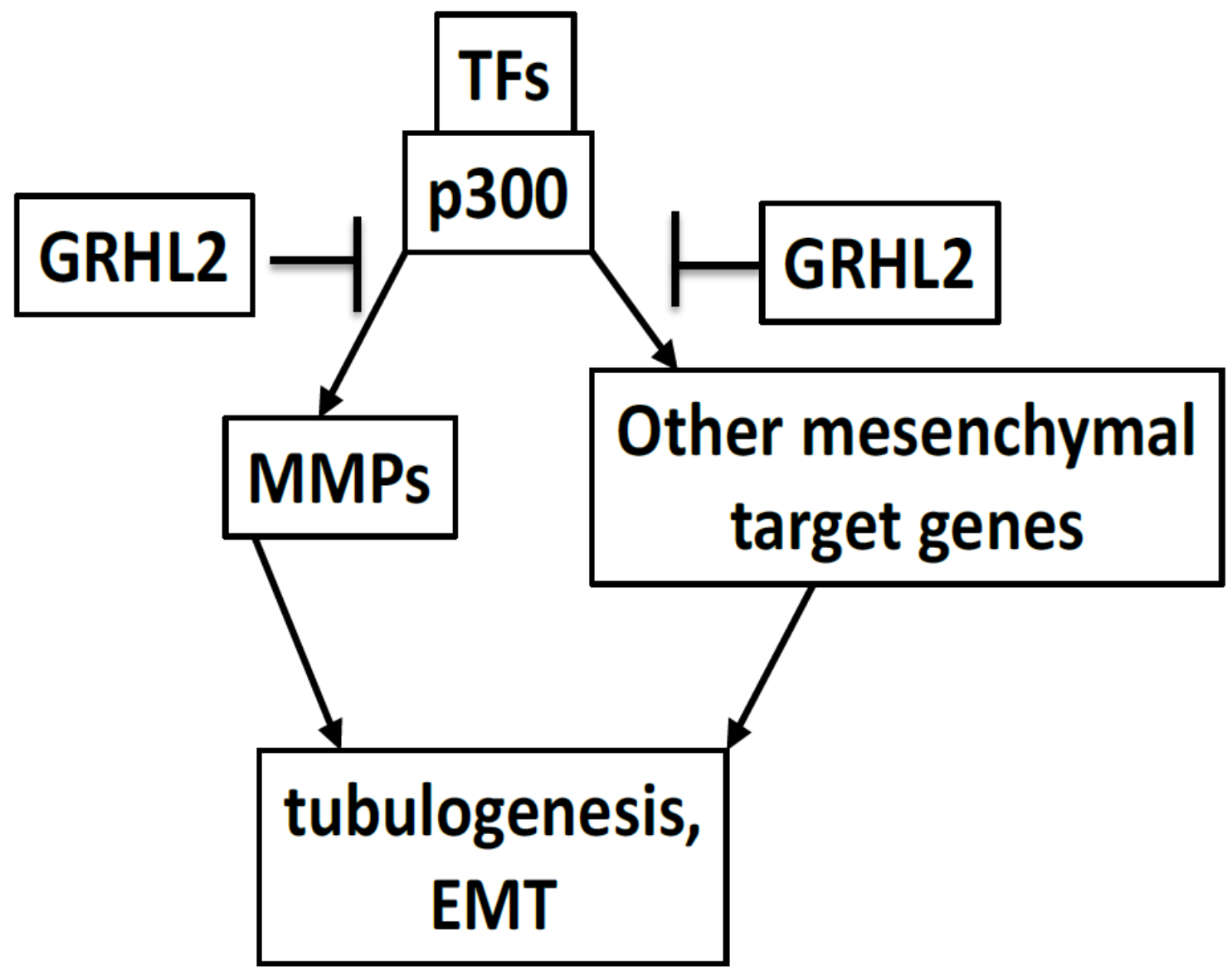

Figure 8 


\section{Supplemental Figure Legends}

Figure S1. GRHL2 shRNA accelerates HGF-induced cell scattering (videomicroscopy). Cell scattering time lapse movies were taken every 15 minutes over a 24 hour period. Time lapse images were obtained using a Nikon Eclipse TE2000-E with Photometrics CoolSNAP HQ2 Monochrome CCD with Phase 40x/0.75 objective. (left panel): MDCK + vector; (right panel): MDCK+GRHL2 shRNA

Figure S2. GRHL2 did not affect Erk or Akt signaling downstream of HGF/Met. Lysates from HGF induction time course using the indicated cell lines was analyzed by western blotting using the indicated antibodies. Densitometry data represent ratios of phospho-Akt or phosphoERK to the corresponding total protein levels.

Figure S3. Effect of GRHL2 on cyst formation. A. Constitutive GRHL2 expression in mIMCD-3 cells prevents tubulogenesis. B. Knockdown of GRHL2 prevents cyst formation in MDCK. (left panel) GRHL2 knockdown by western blotting. (middle panel) Quantification of cysts per well. (right panel) Representative images from MDCK collagen cysts

Figure S4. Knockdown of GRHL2 following cyst formation (inducible shRNA) promotes tubulogenesis. A. MDCK cells with doxycycline inducible GRHL2 shRNA were induced with HGF in the presence or absence of doxycycline to induce GRHL2 shRNA. Cyst extensions were quantified 24 hours after HGF induction. Quantification indicates the number of cysts that did not have extensions at 24 hours. B. GRHL2 does not alter proliferation in MDCK cells. The indicated cell lines were plated at equal density and counted in triplicate wells at three days postplating. 
Figure S5. MMPs are important for kidney tubulogenesis. E13.5 ex vivo mouse kidney cultures were grown for 48 hour with batimastat or solvent control and imaged. Ureteric buds were counted for quantification.

Figure S6. GRHL2 did not affect FOS or JUN family members. FOS, FOSL1, FOSB, JUN, and JUNB mRNA expression levels were determined in the indicated cell lines at one or four hours post-HGF induction via qPCR.

Figure S7. GRHL2 suppresses the p300 pathway. p300 effector and target genes induced by HGF in MDCK cells with GRHL2 shRNA vs. cells with constitutive GRHL2 determined by IPA analysis quantitation of the number of unregulated, HGF up-regulated or HGF down-regulated (red=up-regulated, green=down-regulated, and grey=unregulated)

Figure S8. Validation of p300-dependence of reporter assays using WT vs. p300-nonbinding mutant of E1A. HT1080 cells were cotransfected with the indicated reporters and expression constructs. Values represent relative luciferase activity normalized to TK- $\beta$ galactosidase control.

Figure S9. Further validation of reporter assays: A. c-Myc did not inhibit GAL4-p300 pG5 assay. B. GRHL2 did not inhibit GAL4-VP16 activation of GAL4- responsive element. HT1080 cells were transfected with the indicated reporters and expression constructs. Values represent relative luciferase activity with GRHL2 present compared to empty vector alone normalized to TK- $\beta$-galactosidase control.

Figure S10. GRHL1 and GRHL3 inhibit transactivation by p300. HT1080 cells were cotransfected with the indicated reporters and expression constructs. Values represent relative luciferase activity normalized to TK- $\beta$-galactosidase control. 
Figure S11. GRHL2 suppresses activity of the HAT domain (alone) of p300. Recombinant GRHL2 inhibits recombinant p300 HAT (1283-1673) acetylation of H3K27 in a dose dependent manner in the $\mathrm{H} 3$ acetylation assay. Coomassie stains to assess the quality of recombinant proteins are shown in figure S19.

Figure S12. GRHL1 and GRHL3 inhibit p300 HAT activity. Recombinant GRHL1 and GRHL3 inhibit p300's acetylation of H3K27 in H3 acetylation assay. Coomassie stains to assess the quality of recombinant proteins are shown in figure S19.

Figure S13. GRHL2 did not cause global changes in heterochromatin condensation like E1A. (left panel) GRHL2-lacI-mCherry, E1a-lacI-mCherry or lacI-mCherry expression constructs were transfected into RRE cells and confocal microscopic images of chromatin condensation were quantitated.

Figure S14. Recombinant GRHL2 and p300 pulldown interaction. Recombinant GRHL2 and p300 were assayed for interaction by p300 IP/GRHL2 western blot.

Figure S15. Mapping of GRHL2 domains involved in inhibiting HAT activity in vitro. A. Recombinant GST-GRHL2 fragments were generated without the GRHL2 transactivation domain (136-625), DNA binding domain ( $\Delta 245-494)$, or dimerization domain (1-520) and assayed for inhibition of p300 HAT activity. B. Recombinant GST-GRHL2 fragments were generated within the GRHL2 transactivation and assayed for inhibition of p300 HAT activity. Coomassie stains to assess the quality of recombinant proteins are shown in figure S19. C. Synthetic peptide (aa420-442) and scramble peptide were assayed for inhibition of p300 HAT activity. (right panel) Quantification of H3K27-Ac levels compared to no peptide control. 
Figure S16. Mapping of GRHL2 domains involved in inhibiting transactivation by GAL4p300 in reporter assays. HT1080 cells were cotransfected with the indicated reporters and expression constructs. Values represent relative luciferase activity normalized to TK- $\beta$ galactosidase control.

Figure S17. GRHL2 aa 425-437 important for repressing GLUD1 promoter through p300. HT1080 cells were cotransfected with the indicated reporters and expression constructs. Values represent relative luciferase activity normalized to TK- $\beta$-galactosidase control.

Figure S18. GRHL2 $\Delta 425-437$ interacts with p300 in a co-transfection assay. p300 and wt GRHL2 or GRHL2 $\triangle 425$-437 expression vectors were transfected into 293 cells. Lysates were immunoprecipitated with p300 antibody and probed for GRHL2 interaction.

Figure S19. Confirmation of recombinant proteins (Coomassie blue staining). A.

Prescission cleaved GRHL2 for figure 4A H3 acetylation assay. B. GST-GRHL1 and GST-

GRHL3 for figure S12 H3 acetylation assay. C and D. GST-GRHL2 fragments for figure S15 H3 acetylation assay. E. GST-GRHL2 fragments for figure 6A H3 acetylation assay. F. GSTGRHL2 fragments for figure 6B H3 acetylation assay. 


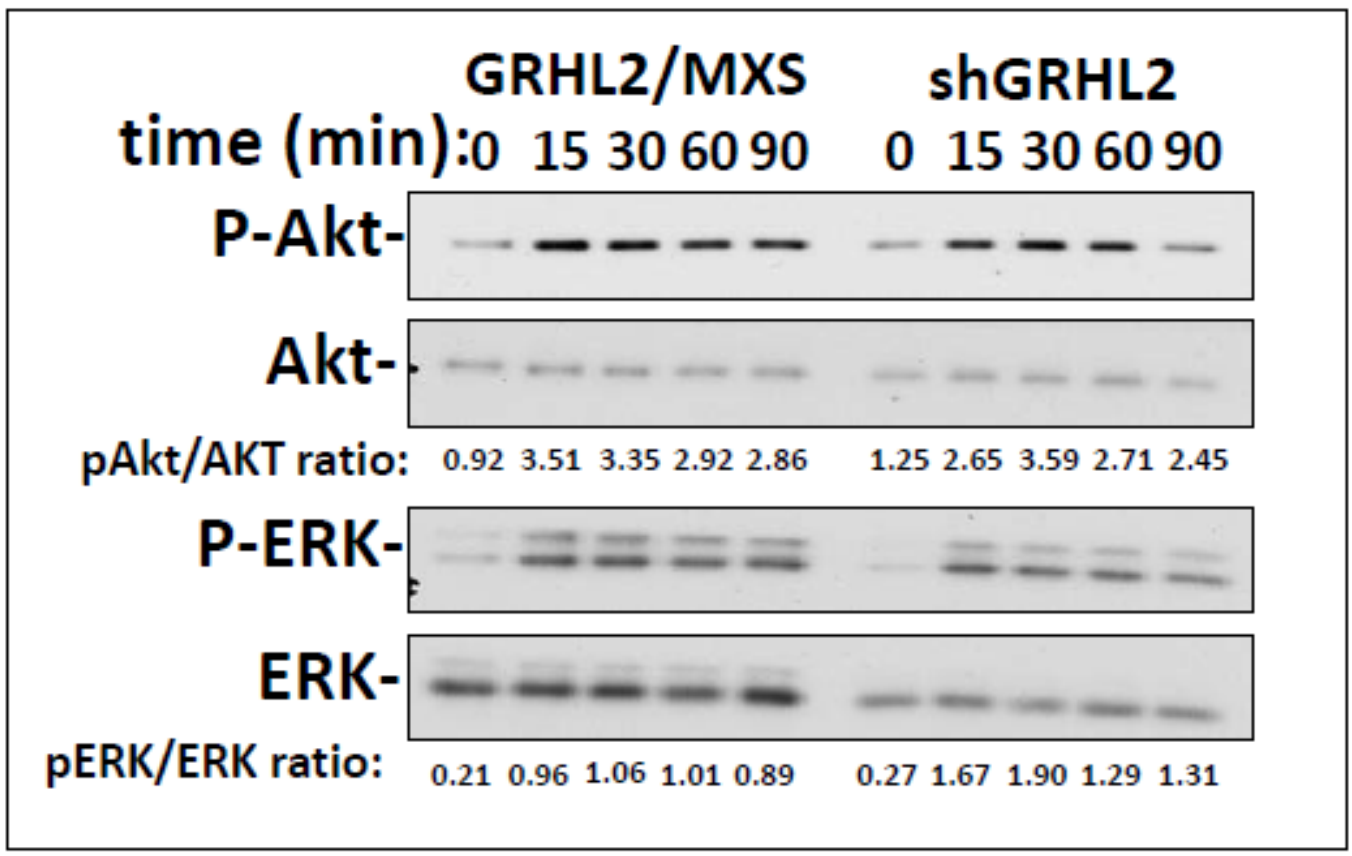

Figure S2 
A.

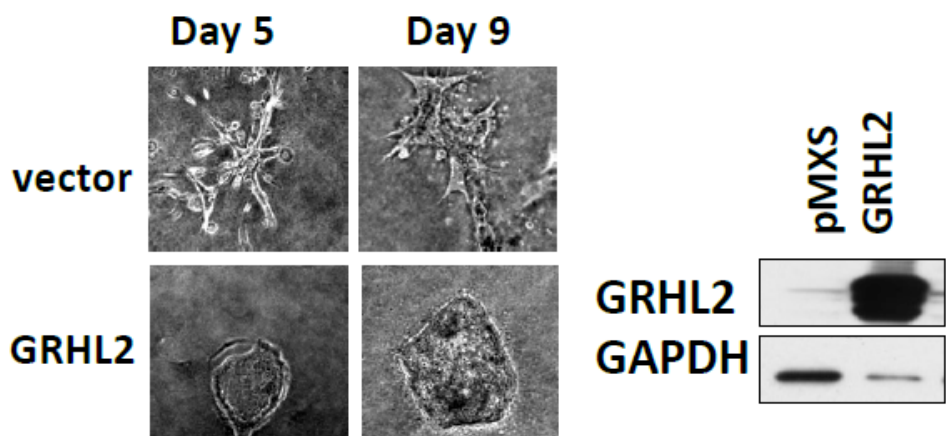

mIMCD3 cells

B.

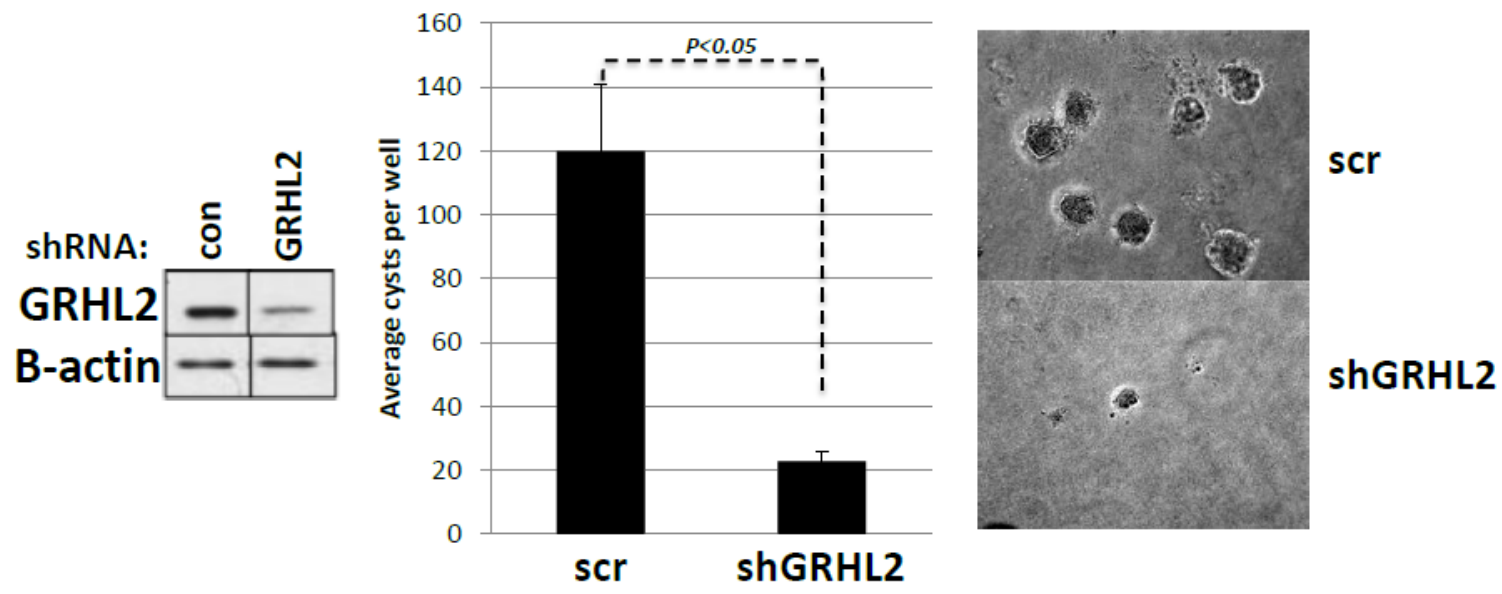

Figure S3 
A.

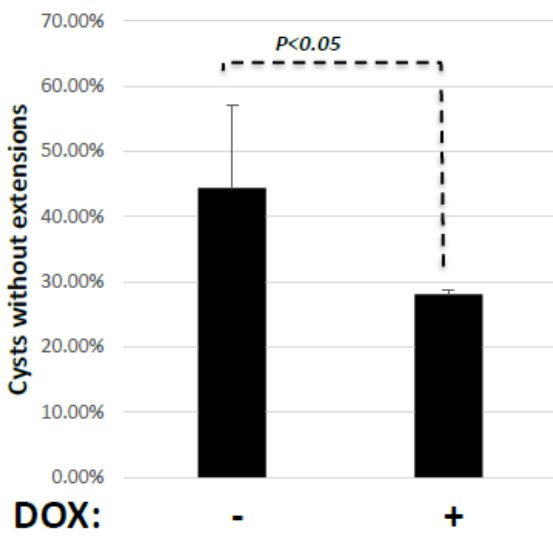

B.

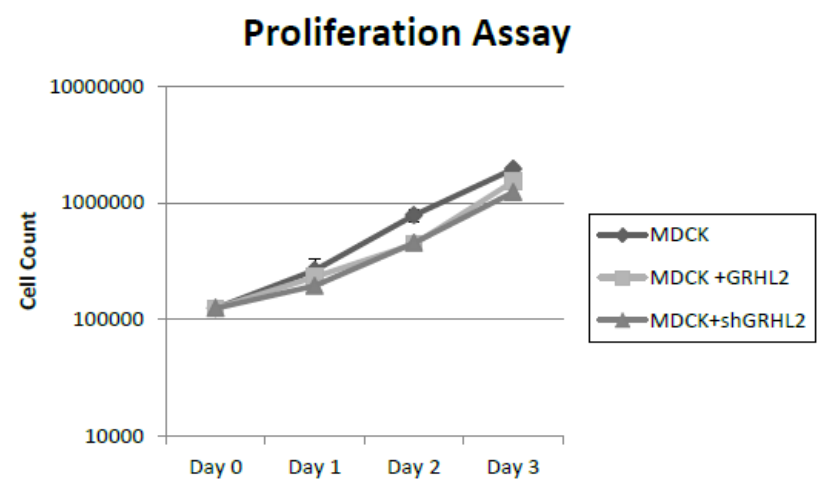

Figure S4 


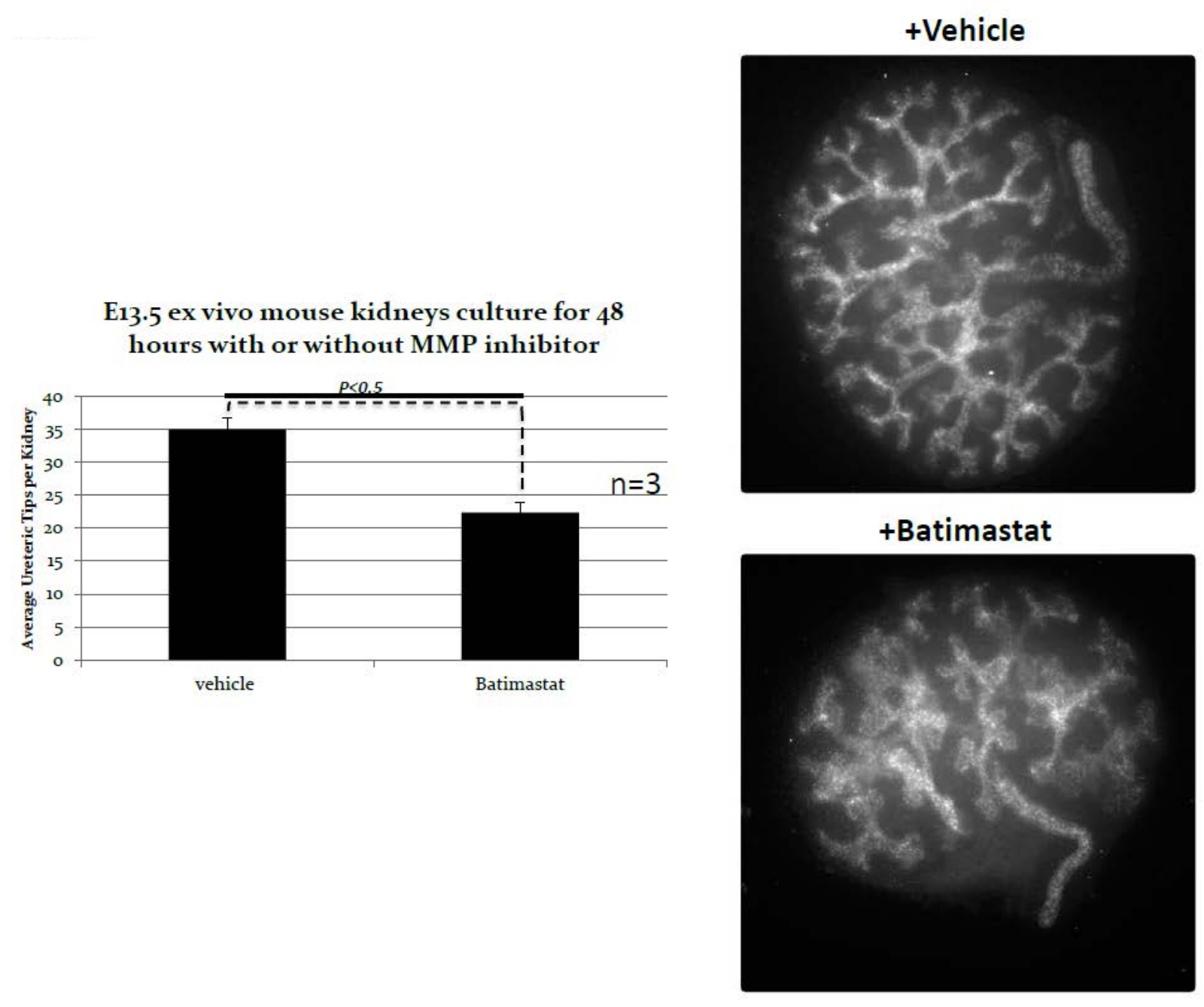

Figure S5 
Figure S6

FOS expression

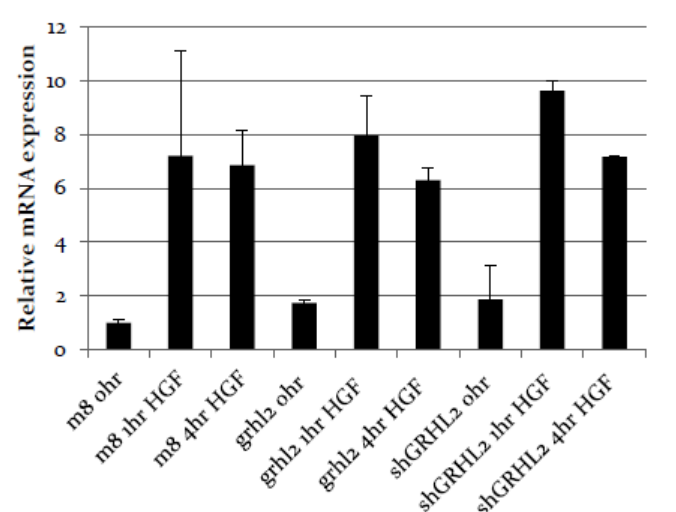

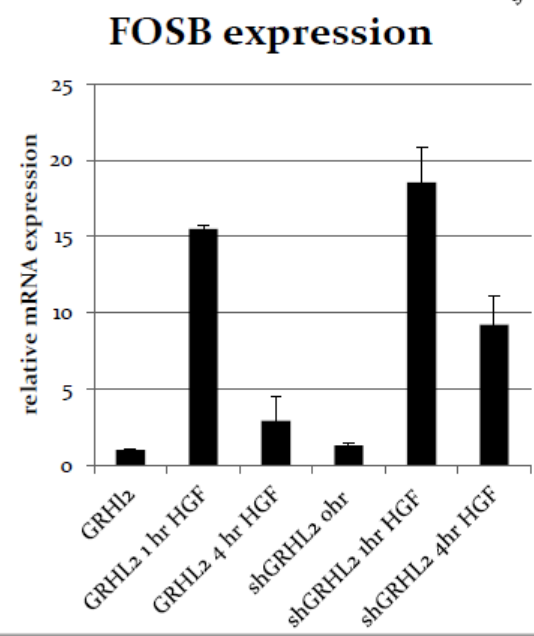
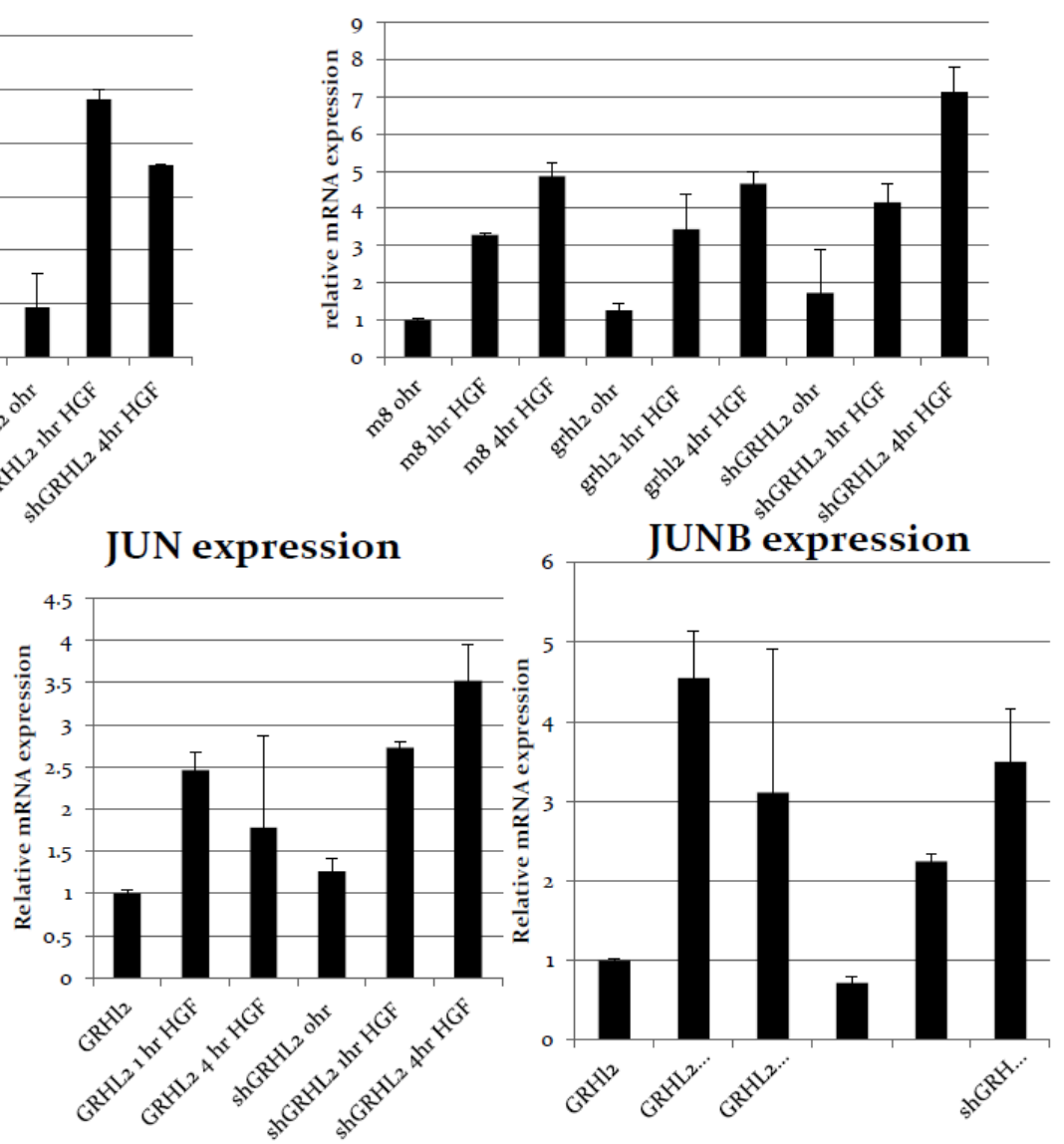

Figure S6 
shGRHL2+HGF/shGRHL2-HGF

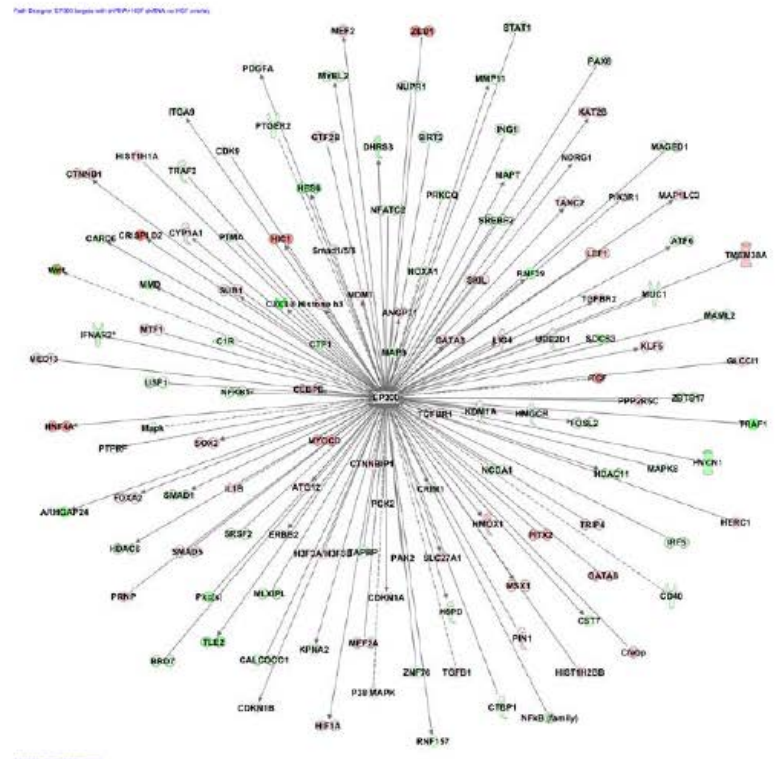

GRHL2+HGF/GRHL2-HGF

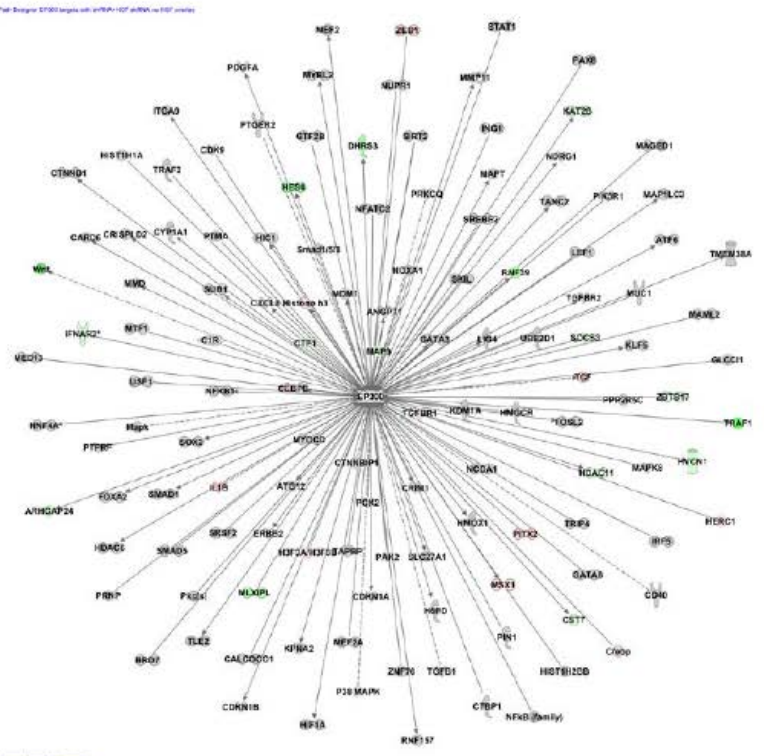

Figure S7 

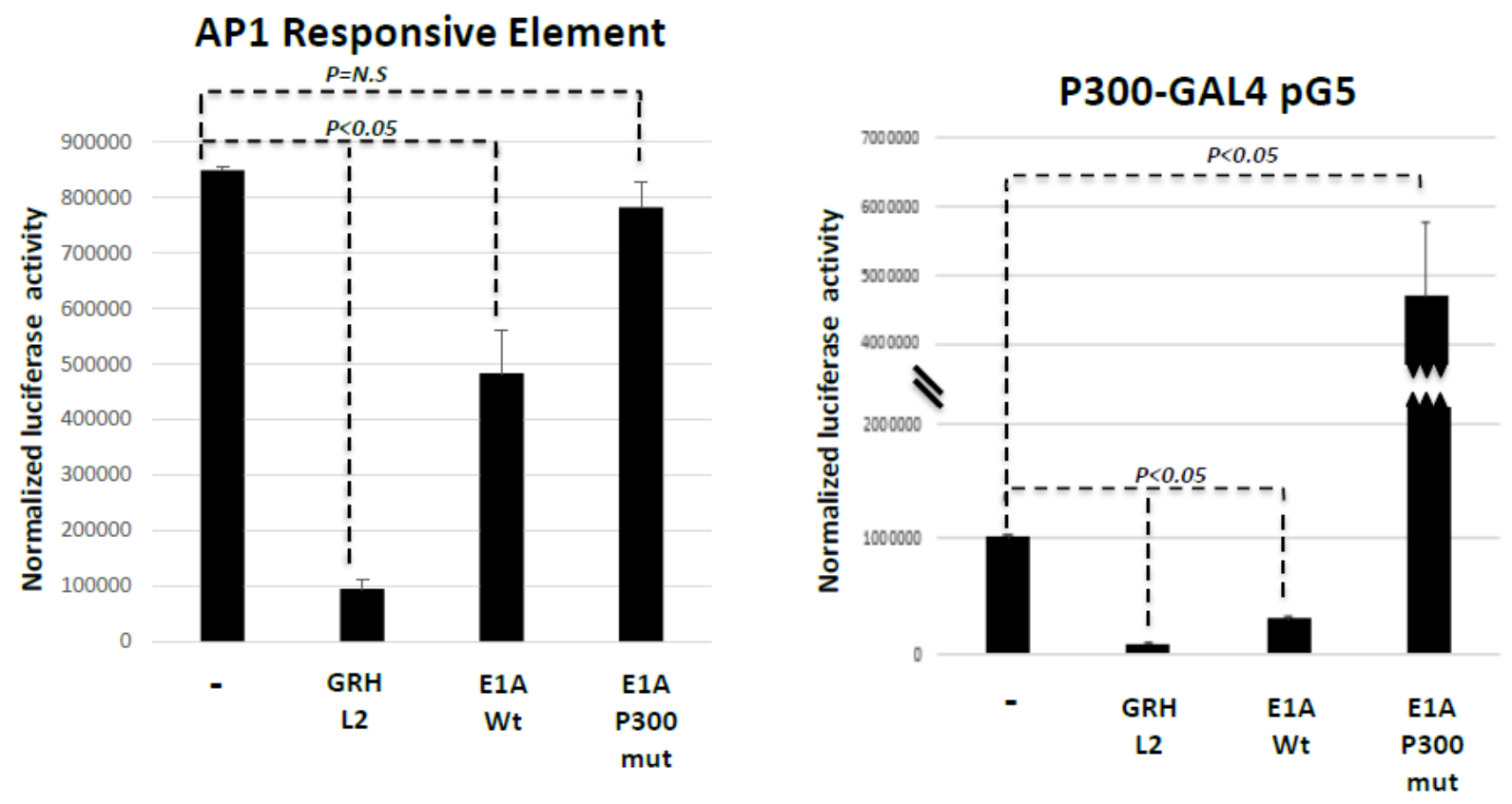

Figure S8 
A.

P300-GAL4 pG5

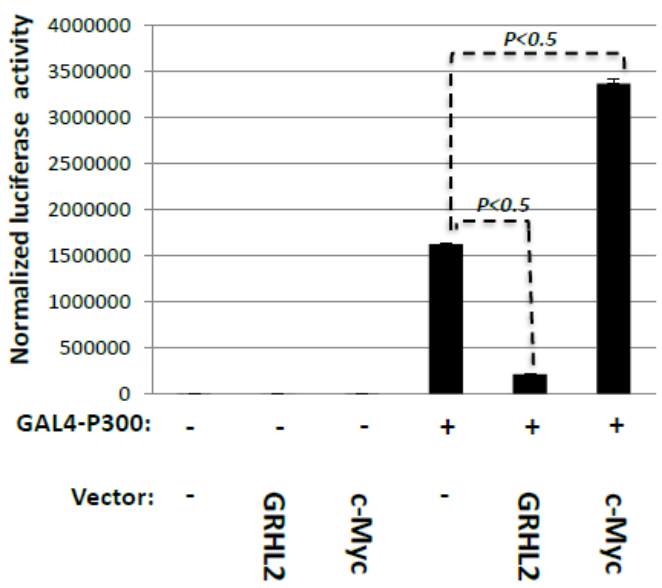

B.

GRHL2/ev-cDNA with internal control
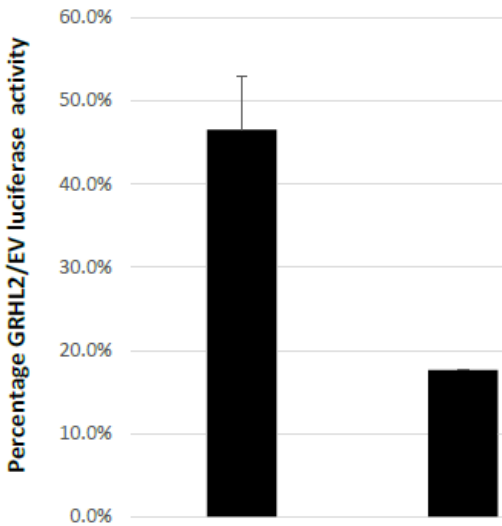

GAL4-

P300

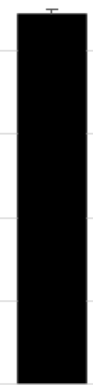

GAL4-

VP16

Figure S9 

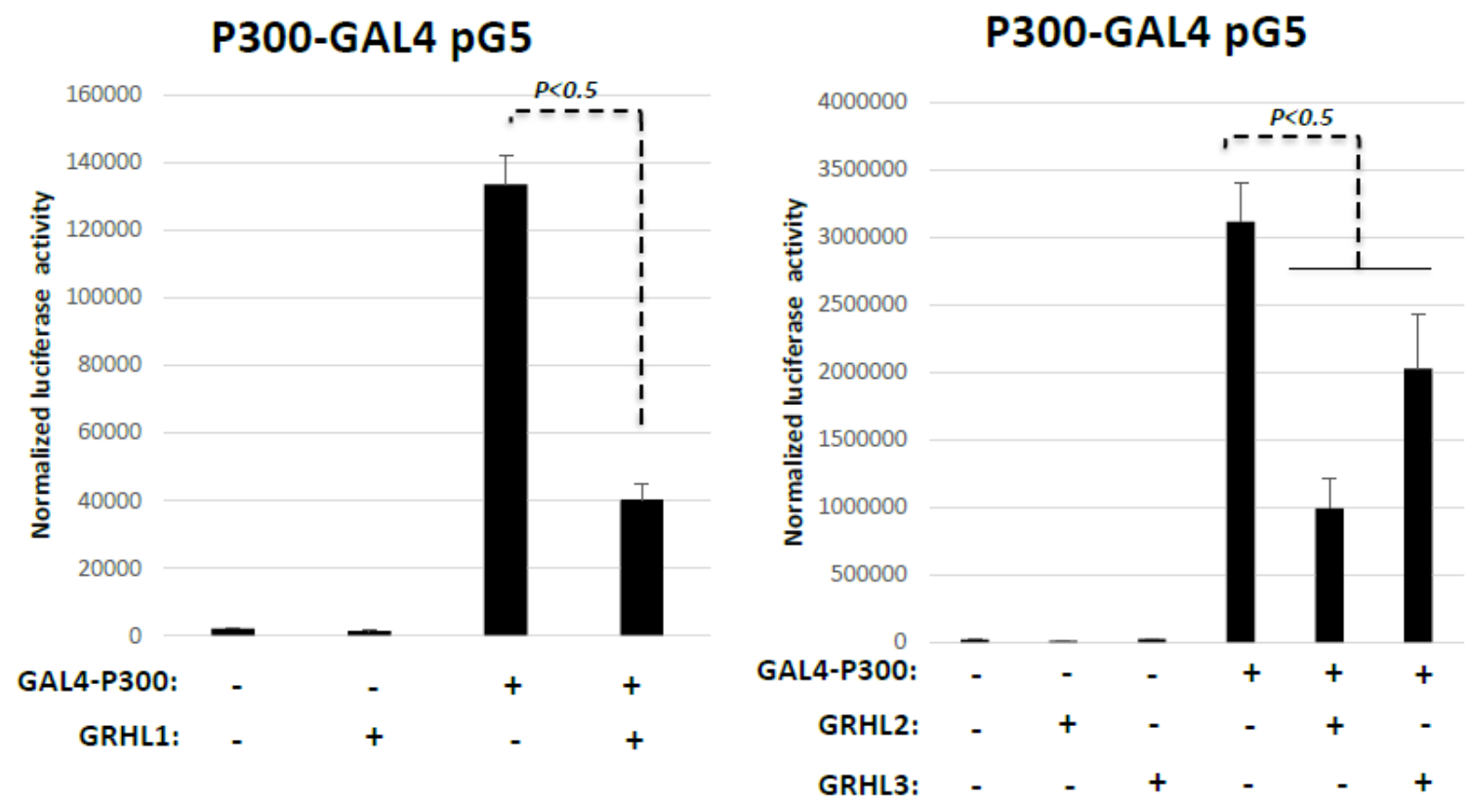

Figure S10 


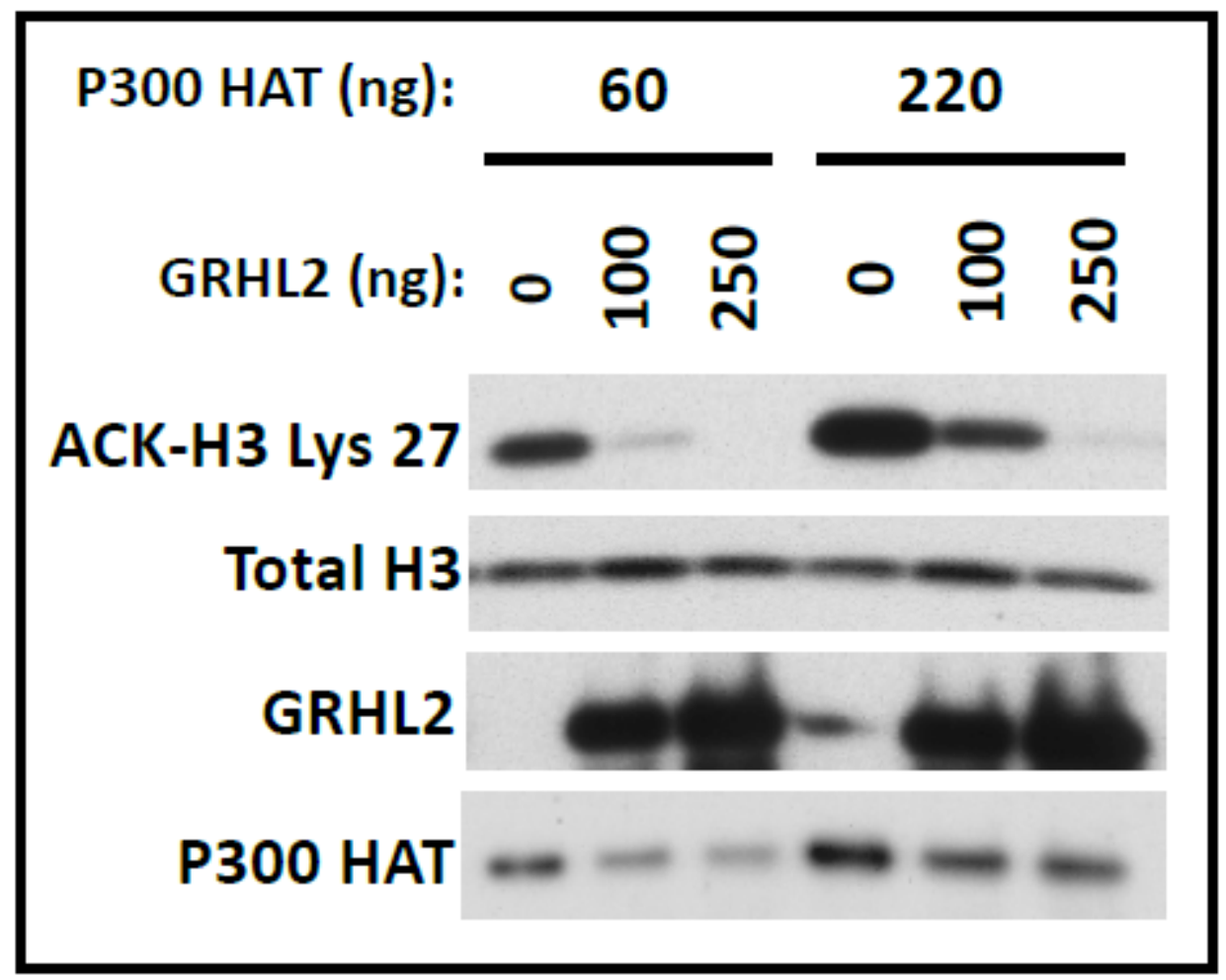

Figure S11 


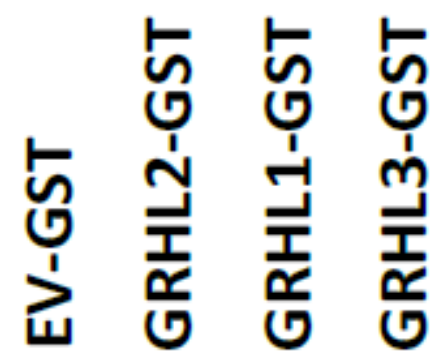

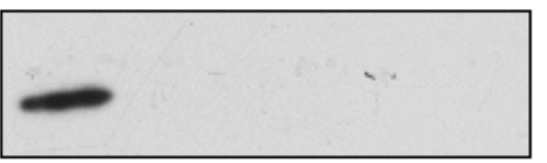

ACK-H3 Lys 27

Figure S12 


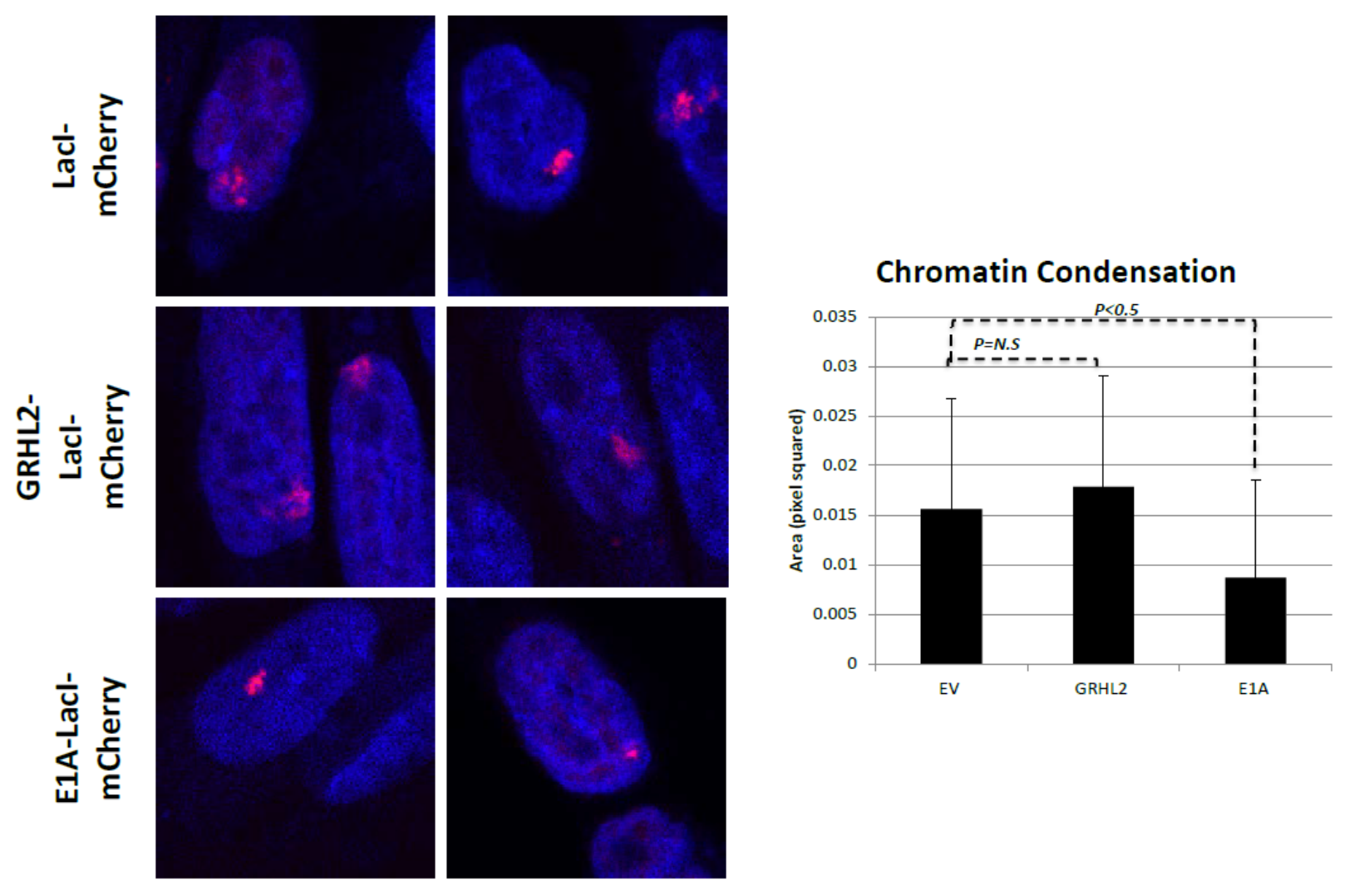

Figure S13 

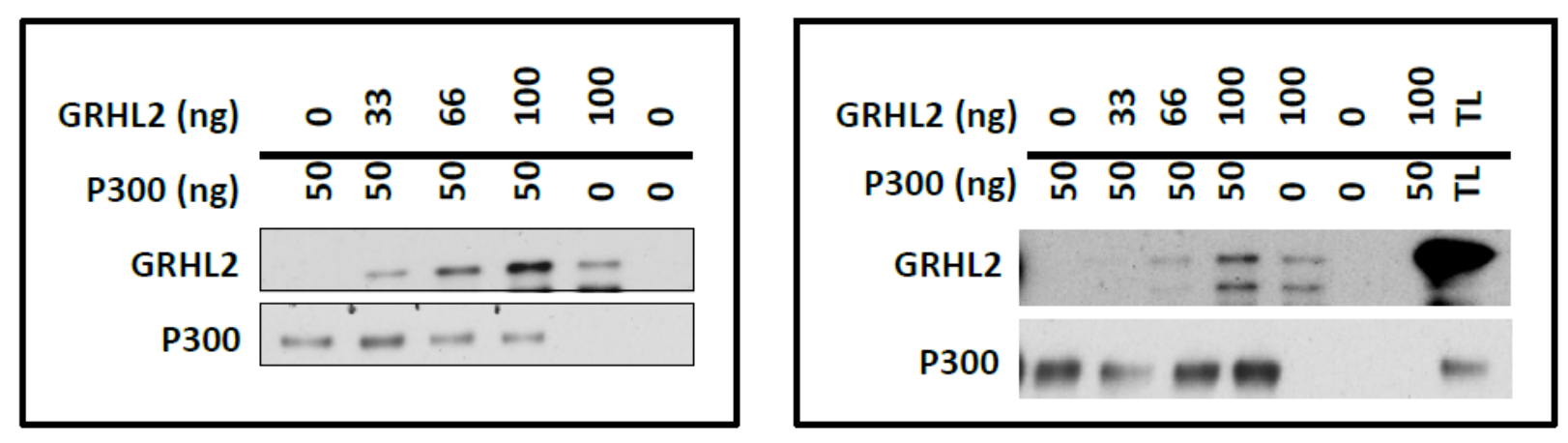

Figure S14 
A.

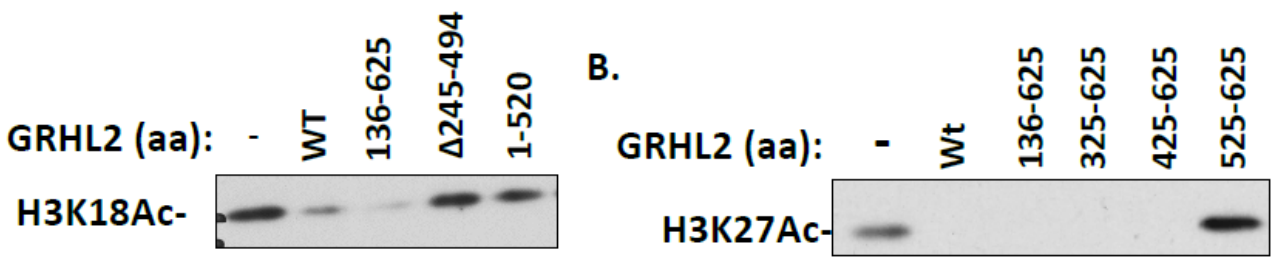

C.
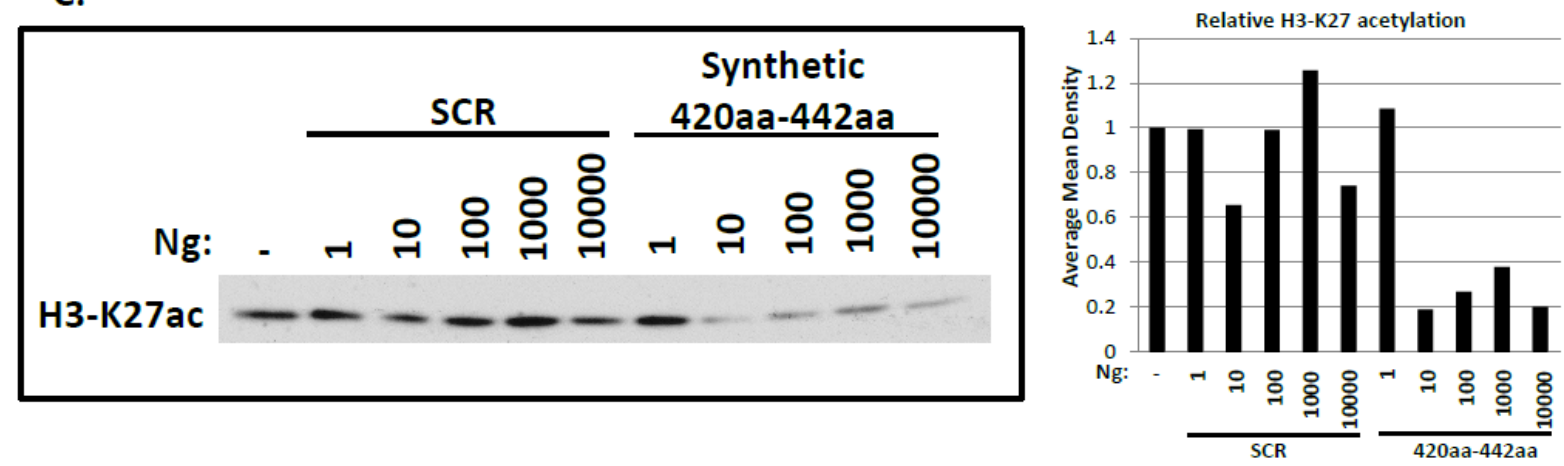

Figure S15 


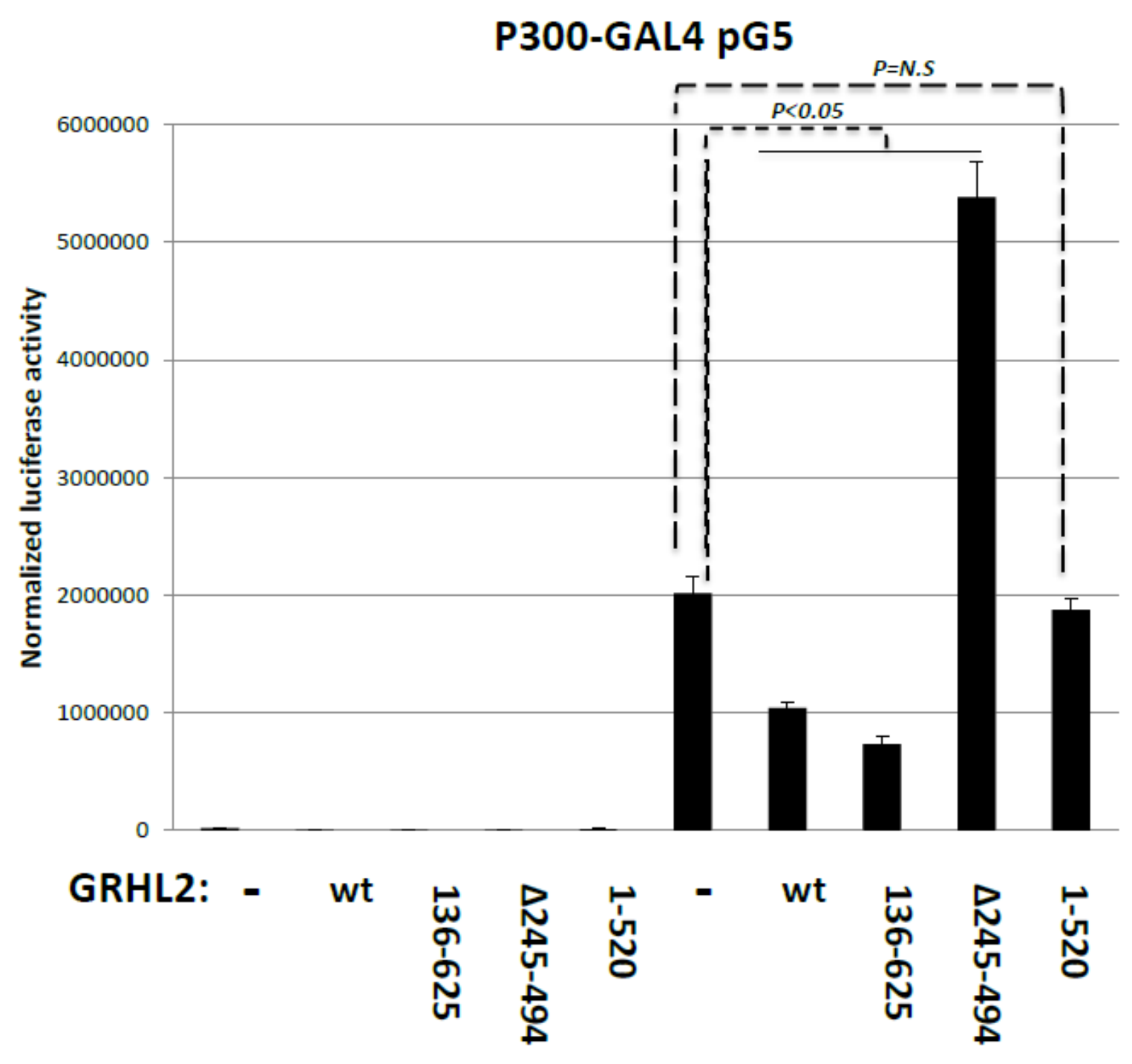

Figure S16 

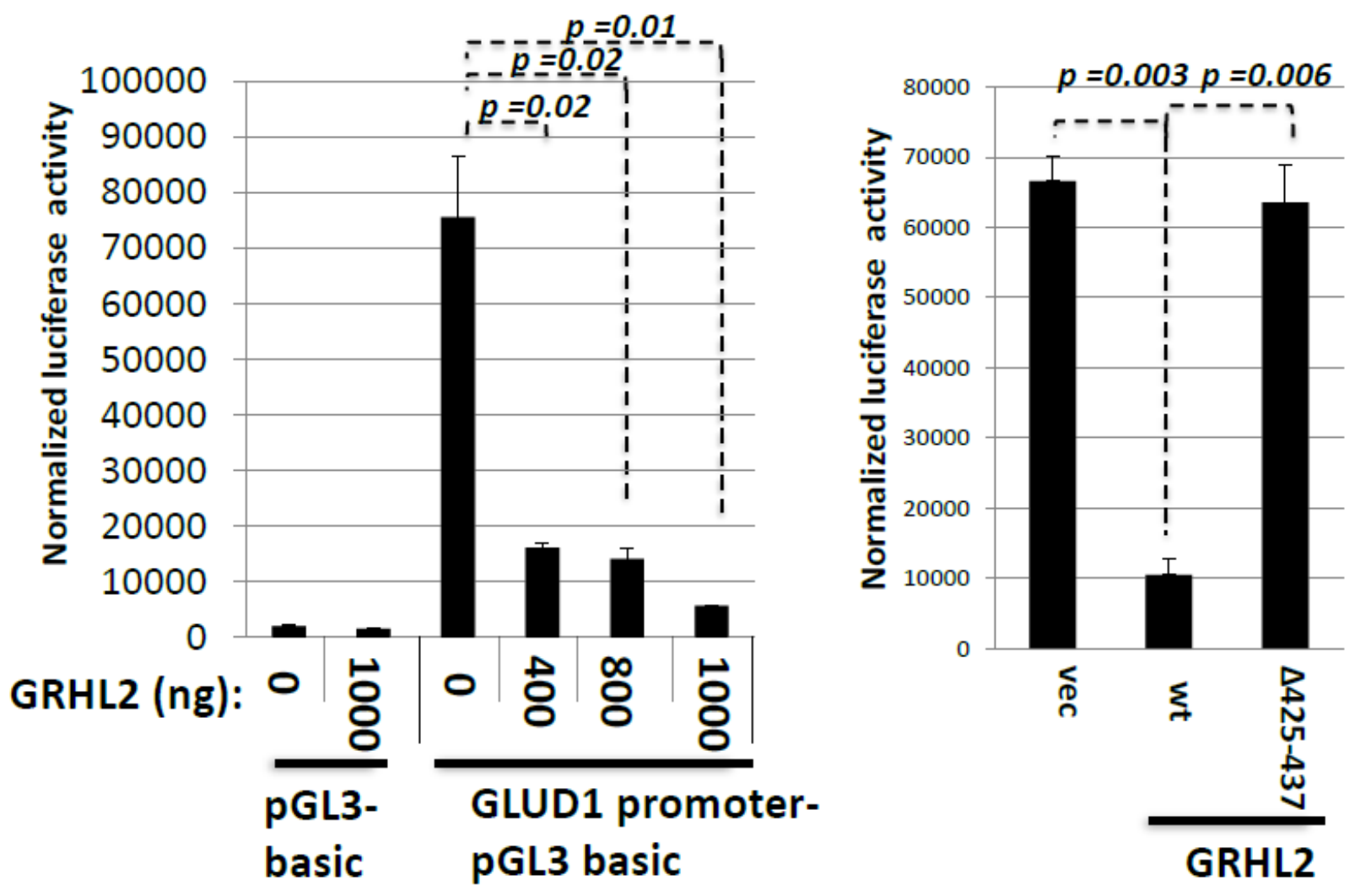

Figure S17 


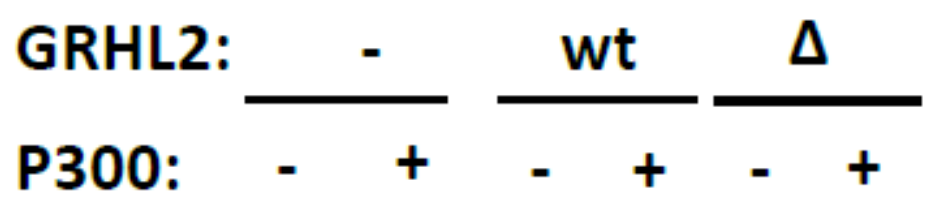

FLAG-GRHL2 TL

FLAG-GRHL2 IP

P300 TL

P300 IP

Figure S18 

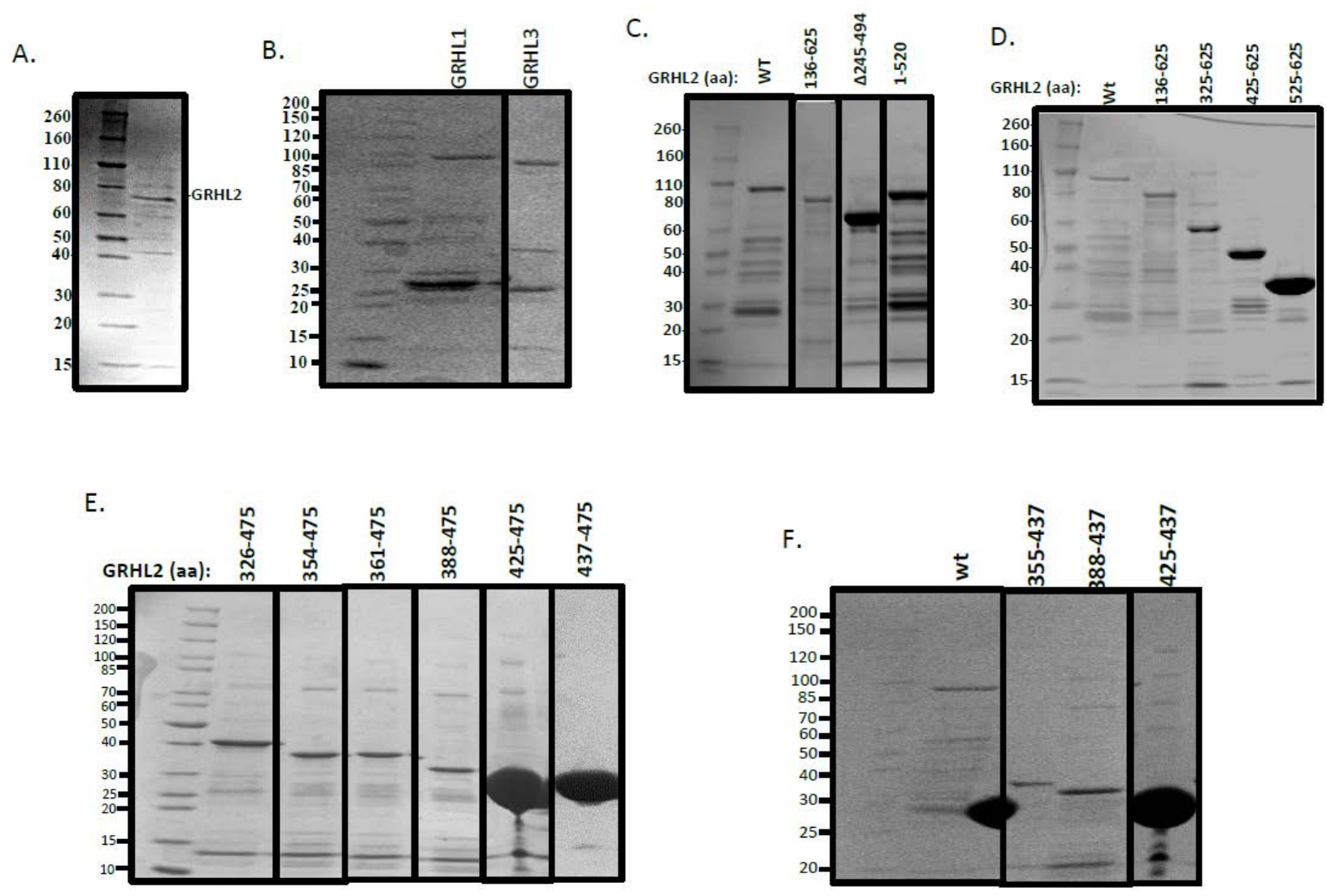

Figure S19 
Chapter 3

\section{The role of GRHL2 in Kidney Cancer and Kidney Development}

The following represents unpublished data. 


\section{$\underline{\text { Introduction }}$}

\section{Kidney Cancer}

As discussed in Chapter 1, GRHL2 suppresses EMT, causes MET, and is important in the developing kidney (Werth et al.; Cieply et al., 2012; Aue et al., 2015). Previously published microarray data (GEO website, GSE15641) shows that GRHL2 is significantly downregulated in clear cell renal cell carcinomas (ccRCC) (figure 1). ccRCC is associated with an EMT phenotype, consisting of gene markers resembling adipogenic and pluripotent mesenchymal stem cell-like differentiated states (Tun et al., 2010). ccRCC overexpresses mesenchymal markers, vimentin and N-cadherin (Tun et al., 2010). Also, TGF- $\beta$ signaling, an EMT driver, is upregulated in ccRCC (Boström et al., 2013). The most common mutation in ccRCC is an inactivating mutation of Von Hippel-Lindau (VHL) (Clark, 2009). Under normoxic conditions, VHL and its E3 ligase complex ubiquitinates hypoxia-induced factor alpha (HIF $\alpha$ ) leading to HIF $\alpha$ 's degradation (An and Rettig, 2005). When VHL is mutated in ccRCC, HIF $\alpha$ accumulates and trans-activates the EMT transcription factors, Twist and Snail (Banumathy and Cairns, 2010). In a breast cancer microarray (GEO website, GSE24202), Twist and Snail are able to downregulate GRHL2 suggesting a mechanism of GRHL2 downregulation in ccRCC (figure 1). These observations led to the hypothesis that GRHL2 would function as a tumor suppressor in ccRCC cell lines and revert the EMT phenotype.

\section{Kidney development}

Kidney development is an intricate process involving morphogenesis, multiple signaling pathways, and cell-cell interactions. This developmental process occurs via the interaction of the ureteric bud and the metanephric mesenchyme. Growth factors originating from the metanephric 
mesenchyme include glial cell derived growth factor (GDNF) and hepatocyte growth factor (HGF). These factors activate their cognate receptors on the ureteric epithelium - RET and MET, respectively (Costantini, 2010). Inductive signaling from the metanephric mesenchyme results in elongation and branching of the numerous ureteric bud tips. At this point, the ureteric bud induces the differentiation of the metanephric mesenchyme into the glomerulus. The metanephric mesenchyme goes on to form the glomerulus, proximal and distal convoluted tubules, and loop of henle. The ureteric bud is derived from the epithelial Wolffian duct and expresses GRHL2 (Werth et al., 2010; Aue et al., 2015). The ureteric bud goes on to form the kidney collecting ducts, calyces, renal pelvis, and ureter. In the adult kidney, GRHL2 is expressed in the collecting duct. As previously noted, GRHL2 is essential for programming the epithelial cell phenotype, partially through activation of various cell-cell adhesion genes, including E-cadherin, claudins, and desmosmal genes (Werth et al., 2010). Therefore, GRHL2 expression leads to tight epithelial cell-cell connections and may be responsible for collecting duct integrity. Also, GRHL2 suppresses the EMT and promotes the reciprocal process, MET (Cieply et al., 2012). Both of these processes are required for kidney development (Faa et al., 2012). Cells undergoing EMT demonstrate increased migration and invasion (Kalluri and Weinberg, 2009). Because GDNF and HGF increase migration, GRHL2 is expected to antagonize these signaling pathways (Uehara and Kitamura, 1992; Tang et al., 1998). Reciprocally, GRHL2 would be inhibited in ureteric bud tip cells allowing the cells to bifurcate into tubules and/or invade in response to GDNF and HGF. To support this hypothesis, ESRP1 ${ }^{-/-}$ knockout mice increase kidney trifurcations (Russ Carstens, personal communications). ESRP1 is a downstream target of GRHL2 and is responsible for some of the epithelial regulatory effects associated with GRHL2 expression (Cieply et al., 2012). The role of GRHL2 as a master 
epithelial programming transcription factor places it in a position to influence all of these processes and signaling pathways. The investigation of GRHL2 in kidney development will elucidate its effect on ureteric bud formation, inductive signaling pathways, ureteric bud tip development, and glomerulus maturation. Furthermore, the examination of GRHL2 and HGF/MET signaling will have implication not only in kidney development, but also cancer progression.

\section{$\underline{\text { Results/Discussion }}$}

\section{Kidney cancer}

To investigate the role of GRHL2 in ccRCC, 786-0, a clear cell renal cell carcinoma cell line lacking functional VHL protein, was utilized (Williams et al., 1978; Jiang et al., 2003). Constitutively expressed GRHL2 in 786-0 cells caused a mesenchymal-epithelial transition based on cell morphology (figure 2A, B). 786-0 cells undergo branching morphogenesis in three dimensional matrigel culture upon HGF induction (Peruzzi et al., 2006; Lee et al., 2008), and GRHL2 prevented HGF-induced branching morphogenesis (figure 2C). Interestingly, this effect led us to examine GRHL2 in MDCK tubulogenesis which resulted in discoveries explored in Chapter 2. Constitutively expressed wild type VHL causes a MET in 786-0 cell (Peruzzi et al., 2006). Since both VHL and GRHL2 reverted ccRCC cells, the cells were examined for anoikis sensitivity which is normally associated with the epithelial phenotype (Frisch and Francis, 1994). Indeed, VHL and GRHL2 restored anoikis sensitivity to 786-0 cells (figure 2D). These results suggested that GRHL2 was a bona fide tumor suppressor in the context of ccRCC since it caused a MET, restored anoikis sensitivity, and decreased invasion. 
The next step was to demonstrate that GRHL2 depletion in normal kidney epithelium increased EMT, anoikis resistance, and invasion. It is a widely held view that ccRCC is derived from the proximal tubule of the kidney (Büttner et al., 2015). Human kidney proximal tubular epithelial cell lines, HKC-8 and HK-2, were used to perform GRHL2 shRNA depletion experiments. Surprisingly, when HKC-8 and 786-0 cells were compared to HMLE cells using qPCR, there was no detectable level of GRHL2 mRNA in the kidney cells (figure 3A), even though HKC-8 cells are classified as proximal tubular epithelial cells. Next, a panel of kidney cell lines were tested for GRHL2 expression with HMLE cells used as a positive control (figure 3B). HKC-8 cells displayed a protein band that migrated significantly higher than normal human GRHL2. The protein band was resistant to three GRHL2 shRNAs, suggesting the band was not GRHL2 when examined in conjunction with the qPCR data (figure 3A,C). Both HK-2 cells and miMCD cells did not express GRHL2 (figure 3B). However, Madin-Darby canine kidney cells (MDCK) expressed high levels of GRHL2, and MDCK cells are derived from canine collecting duct cells (Gekle et al., 1994). The developmental process of the kidney clearly explains why HKC-8 and HK-2 cells do not express GRHL2, but MDCK cells do (See Chapter introduction for full description). During kidney development, the ureteric epithelium (positive for Ecadherin and GRHL2) interacts with the metanephric mesenchyme (negative for E-cadherin and GRHL2) to form the adult kidney. The ureteric epithelium give rises to collecting duct cells from which the MDCK cell line was derived, thereby explaining the GRHL2 positivity of MDCK cell. However, HKC-8 and HK-2 cells were derived from the proximal tubule cells originating from the metanephric mesenchyme and do not express GRHL2.

Therefore, we were constitutively expressing GRHL2 in ccRCC cell lines that never expressed GRHL2 under physiological conditions, making the clinical relevance of GRHL2 as 
tumor suppressor in ccRCC questionable. It should be noted that the initial flaw in the experiment arises in the published microarray data comparing normal kidney tissue and ccRCC. When heterogeneous tissue samples are collected, genes present in only one cell population may appear highly regulated. In this specific case, kidney collecting ducts express high levels of GRHL2, but the other mesenchymal originating kidney cells have no detectable GRHL2 levels. Therefore when ccRCC expands, the collecting duct cells are not amplified, causing the microarray to appear as if GRHL2 was downregulated.

Even though our results suggest that GRHL2 is not a tumor suppressor in ccRCC, our results do have significant implication in the diagnosis of ccRCC. GRHL2 regulates ESRP1/2 (See Chapter 1 EMT section) which is responsible for the alternative splicing of fibroblast growth factor receptor 2 (FGFR2) isoforms (Warzecha et al., 2009). The mesenchymal FGFR2 isoform IIIc (FGFR2-IIIc) is only expressed in metanepheric mesenchyme derived cells whereas the epithelial FGFR2 isoform IIIb (FGFR2-IIIB) is only expressed on ureteric epithelial cells. GRHL2 upregulates FGFR2-IIIb presumably by its regulation of ESRP1 and subsequent FGFR2 splicing (figure 4). In a study with significant clinical implications, Zhao et al. examined 470 ccRCC specimens and 68 normal kidney specimens for alternative splicing events (Zhao et al., 2013). In this cohort, $~ 90 \%$ of ccRCC expressed FGFR2-IIIc compared to the normal kidney tissue expressing FGFR2-IIIb. The 10\% of ccRCC cells exhibiting the FGFR2-IIIb isoform were associated with higher patient survival, lower tumor grade, and decreased tumor size. More importantly, when these FGFR2-IIIb isoform positive tumors were examined by a pathologist, the tumors were re-classified as chromophobe renal cell carcinoma or papillary renal cell carcinomas. FGFR2-IIIb isoform, ESRP1, and GRHL2 all correlated with these less invasive renal cell carcinomas (Zhao et al., 2013). The data suggest that GRHL2 expressing cells do not 
become ccRCC, and GRHL2 could be useful as a biomarker for predicting clinical kidney carcinoma subtypes in undiagnosed renal cell carcinoma.

\section{Kidney Development}

An RNA-Seq from MDCK cells with GRHL2 constitutively expressed or depleted in the presence or absence of HGF was previously performed (Chapter 2). Interestingly, several genes with known or implied significance in kidney development, whose HGF regulation was modulated by GRHL2, were identified (Table 1,2). These results, consistent with the MDCK data (Chapter 2), suggested that GRHL2 could play a significant role in kidney development.

Because both GDNF/RET and HGF/MET signaling pathways are able to induce scattering (Uehara and Kitamura, 1992; Tang et al., 1998), GDNF and HGF were investigated for their ability to downregulate or inactivate GRHL2. When MDCK with constitutive RET expression were treated with HGF and/or GDNF, GRHL2 protein was downregulated (figure 5A). Also, GDNF and HGF were both able to downregulate the GRHL2 promoter in promoterluciferase reporter constructs (figure 5B).

Since HGF/MET and GDNF/RET pathways play an important role in kidney development and regulate GRHL2, the effect of GRHL2 on kidney development was examined in the murine model. Since GRHL2-/- mice die at E11.5 from neural tube defects precluding them from kidney examination, GRHL2 ${ }^{+/}$mice were examined for kidney abnormalities. GRHL2 ${ }^{+/}$mice survived to adulthood with no apparent differences in weight, pregnancy, feeding, urine output, or serum BUN levels. Our preliminary data suggested that GRHL2 ${ }^{+/}$mice have significantly less GRHL2 protein compared to their GRHL2 ${ }^{+/+}$littermates (figure 6A). Because ureteric epithelium stability and ureteric tip bifurcation may be affected by depletion of GRHL2, the number of nephrons 
generated in the adult kidney between $\mathrm{GRHL2}^{+/+}$and $\mathrm{GRHL}^{+/-}$mice was examined. P30 GRHL2 $^{+/+}$and GRHL2 ${ }^{+/}$kidneys were paraffin-embedded, sectioned, H\&E stained, and examined for glomeruli $/ \mathrm{mm}^{2}$. There was a $10-30 \%$ decrease in glomeruli numbers in GRHL2 ${ }^{+/-}$kidneys compared to GRHL2 ${ }^{+/+}$kidneys (figure 6B). In GRHL2 ${ }^{+/+}$and GRHL2 ${ }^{+/}$mice, a ViewRNA ISH Tissue 2-plex assay system was used to examine RET and GRHL2 localization in paraffin sections of E15.5 kidneys (figure 7). RET staining was localized to ureteric tips, and GRHL2 was present in all the ureteric epithelium. Both GRHL2 and RET co-localized in the ureteric tips, and no difference in GRHL2 RNA levels was detected between GRHL2 ${ }^{+/}$and $\mathrm{GRHL}^{+/-}$mice. These results were promising, but needed to be further examined in an ureteric specific GRHL2 $2^{-/-}$mouse line.

GRHL2 ${ }^{\text {Flox/Flox }}$ mice (from collaborator Stephan Jane, Monash University) were crossed with HOXB7promoter-cre-EGFP mice (from collaborator Carlton Bates), a promoter with ureteric epithelial specific expression (Zhao et al., 2004). These mice were used to examine the role of GRHL2 in ureteric bud formation and ureteric tip branching. Embryonic kidneys were examined at E18.5 or E13.5 with 48 hour ex vivo culture for ureteric tips quantification (figure 8). Surprisingly, there was no difference between ureteric tips in control and GRHL2 ${ }^{\mathrm{UB}-{ }_{-}}$mice. Also, there was no renal agenesis observed in D30 GRHL2 ${ }^{\mathrm{UB}-{ }_{-}-}$mice which was expected (data not shown).

After these unexpected results, the GRHL2 ${ }^{\text {flox/flox }}$ model was examined to ensure GRHL2 was deleted in the ureteric bud. The Jane lab GRHL2 $2^{\text {flox/flox }}$ model had placed the two lox P sites around exon 2 to create a GRHL2 frameshift mutation rendering the protein nonfunctional. However, after detailed analysis of murine Grhl2 mRNA by Peter Stoilov, it was discovered that Grhl2 mRNA has ATG start codons in exons 1, 2, and 4. GRHL2 exon 4 ATG start codon is the 
strongest match to a Kozak sequence, and GRHL2 exon 1 and 2 ATG start codons were not good matches, suggesting the start sequence could be leaky and skipped in preference for the exon 4 ATG start codon. Therefore, when the Jane GRHL2 ${ }^{\text {flox/flox }}$ mice excises exon 2, the exon 1 open reading frame is only 48 nucleotides which can cause either reinitiation of translation downstream at exon 4 or entirely bypass exon 1 and start translation at exon 4 . The initiation of GRHL2 at exon 4 would result in a $57 \mathrm{kDA}$ Grhl2 protein with intact DNA binding and dimerization domains. To examine if this phenomenon was occurring in the GRHL2 ${ }^{\text {floxfllox }}$ mice, kidneys were harvested, and primary cells were generated. After the kidney epithelial cells were sorted with Dolichos Biflorus Agglutinin (DBA), cells were infected with adenovirus-Cre initiating the deletion of exon 2. GRHL2 western blotting clearly shows the creation of a 57kDA GRHL2 isoform upon adenoCre addition that corresponds to initiation from the exon 4 start codon (figure 9). This data, in conjunction with the phenotype observed in the GRHL2 ${ }^{\mathrm{UB}-/ /}$ mice, suggested that a functional GRHL2 protein was being produced, and our GRHL2 ${ }^{\mathrm{UB}-{ }^{-}-}$mice were not useful for examining the role of GRHL2 in kidney development.

During the period of time this research was occurring, Schmidt-Ott et. al. published “Grainyhead-Like 2/Ovo-Like 2 Pathway Regulates Renal Epithelial Barrier Function and Lumen Expansion”(Aue et al., 2015). In this Schmidt-Ott study, it was demonstrated that global Grhl2 ${ }^{-/-}$mice result in decreased collecting duct luminal expansion at E10.5 (Supported by Chapter 2), and Grhl2 depletion in miMCD cells decreases luminal expansion in three dimensional culture and affected epithelial barrier formation (Aue et al., 2015). Also, Grhl2 binds and upregulates OVOL2, an epithelial transcription factor (Roca et al., 2013), which is able to rescue the epithelial barrier and luminal expansion defects in the miMCD cells. Subsequently, in an abstract, the Schmidt-Ott laboratory saw renal agenesis in $6.8 \%$ of 
ureteric bud specific GRHL2\%- mouse (excising GRHL2 exon 4) and a reduction in kidney size, but these results have not been published to date. The Schimdt-Ott laboratory used the Hoxb7cre+ mouse generated by McMahon et. al. (Yu et al., 2002) which has lower penetrance than the Hoxb7cre+EGFP mouse generated by Bates et. al. (Zhao et al., 2004). If the Schmidt-Ott GRHL2 $2^{\text {flox/flox }}$ mouse was crossed with the Bates Hoxb7cre+EGFP mouse, the role of GRHL2 in kidney development would be better understood, and possibly a more severe kidney phenotype would be observed.

\section{Material and Methods}

Cell lines

786-0 and 786+VHL cells (Donald Bottaro, NCI Bethesda, MD) were cultured in RPMI1640 (Invitrogen) with 10\% fetal bovine serum (FBS) (Hyclone) and 1x penicillin-streptomycinglutamine (PSG) (Invitrogen). Human proximal tubular epithelial cell line (HKC clone 8) (Youhua Liu, University of Pittsburgh) and miMCD-3 cells (ATCC) were cultured in advanced DMEM:Ham’s F12 (Gibco) with 10\% FBS and 1x PSG. MDCK cells (Clone M8 described previously in Frisch et al. (Frisch and Francis, 1994) and MDCK+c-RET (Greg Dressler, University of Michigan, Ann Arbor, Michigan) were cultured in advanced Dulbecco's Modified Eagle’s Medium (DMEM) (Gibco) with 10\% FBS (Hyclone) and 1x PSG (Invitrogen). Human proximal tubule kidney cells (HK-2) (ATCC) were maintained in Keratinocyte Serum Free Medium (Invitrogen) with $0.05 \mathrm{mg} / \mathrm{ml}$ bovine pituitary extract, $5 \mathrm{ng} / \mathrm{ml}$ human epidermal growth factor, and 1x PSG (Invitrogen). HMLE culture methods were described previously (Cieply et al., 2013). 
Generation of stable cell lines by retroviral transduction and lentiviral transduction

Full-length GRHL2/pMIG, GRHL2/pMXS and GRHL2 shRNA/GIPz were described previously

(Cieply et al., 2012; Farris et al., 2016).

Western Blotting

Western blotting was performed as described in chapter 2. Primary antibodies used were:

GRHL2, rb (Sigma); $\beta$-actin, ms (Thermo-pierce); and GAPDH, ms (Origene).

\section{Cell Imaging Assays}

786-0 cells images were obtained using a Zeiss Axiovert 200M, Axiocam MRM camera, Phase 20x/0.55, RT, Axiovision Rel. 4.8 software.

Matrigel branching morphogenesis assay

Matrigel was thawed overnight at $4^{\circ} \mathrm{C}$. Undiluted matrigel $(40 \mu \mathrm{l})$ was added to 8 well chamber slides and allowed to solidify for 15 minutes. 786-0 cells were trypsinized, counted and resuspended at 25,000 cells/ml. The cell solution was mixed 1:1 with DMEM Complete with 4\% matrigel solution with or without 320ng/ml HGF, and 400ul of this mixed solution was pipetted on top of undiluted matrigel. The cells were refed every four days with DMEM complete with 4\% matrigel with or without HGF. Branching morphogenesis images were obtained using a Zeiss Axiovert 200M, Axiocam MRM camera, Phase 20x/0.55, RT, Axiovision Rel. 4.8 software.

Anoiks Assay

Anoikis assay was performed as previously described (Cieply et al., 2012). 
Quantitative reverse transcription PCR analysis

HMLE, HKC, and 786-0 cell were plated on $60 \mathrm{~mm}^{2}$ dishes. Cells were harvested using RNAeasy Plus mini kit (Qiagen), analyzed using Nanodrop, and converted to cDNA using SuperScript III First-Strand Synthesis SuperMix (Invitrogen) with oligo dT primers and 1ug of RNA. cDNA was analyzed using SYBR Green PCR Master Mix on an Applied Biosystems 7500 Real Time PCR System. Values reported were determined using the delta delta Ct method using human GAPDH as internal control. Primers used: GRHL2-F

(GCGCCTATCTCAAAGACGAC), GRHL2-R (GGCTTCCAGGGTGTACTGAA), GAPDH-F (TGCCAAAGCCTAGGGGAAGA), GAPDH-F (ATGGTTGCCACTGGGGATCT),

RNA-Seq Assay

RNA-Seq was previously described in Chapter 2 material and methods.

GDNF and HGF Cell Scattering Assays

MDCK $+c-R E T$ cells were plated on collagen coated 6 well dishes to give $25 \%$ cell confluency on HGF and/or GDNF induction. Cells were treated with 60ng/ml recombinant human HGF (RD Systems) and/or 50ng/ml GDNF (RD Systems) in DMEM media for the 32 hours. The cells were harvested for protein. Western blotting was performed as previously described in chapter 2 .

\section{Luciferase Reporter Assays}

MDCK $+c-R E T$ were transiently transfected using Lipofectamine 2000 (Invitrogen) at a 1ug DNA: 3ul Lipofectamine ratio. After 20 minute incubation in $300 \mu \mathrm{l}$ of Opti-MEM (Invitrogen), a total of 1.0ug of DNA was transfected into a 12 well with DMEM media and 
refeed 4 hours later with DMEM contain 60ng/ml recombinant human HGF (RD Systems) and/or 50ng/ml GDNF (RD Systems). After 28-36 hours post-transfection, the cells were washed twice with cold PBS, lysed in $200 \mu \mathrm{l}$ of 1x Cell Culture Lysis Buffer (Promega), frozen for at least 1 hour at -80 degrees, thawed on ice, and centrifuged at 13,200rpm. The supernatants were assayed for luciferase activity (Promega) and $\beta$-galactosidase activity as internal control (2x $\beta$-galactosidase assay reagent, $200 \mathrm{mmol} / \mathrm{l}$ sodium phosphate, $\mathrm{pH} 7.3,2 \mathrm{mmol} / \mathrm{L} \mathrm{MgCl}_{2}$, 100mmol/L 2-mercaptoethanol, and 1.33mg/mL o-nitrophenylgalactoside). GRHL2 promoter luciferase reporter plasmids were described previously (Cieply et al., 2013).

\section{FGFR2 splicing assay}

MDCK parental, MDCK+GRHL2/pMXS, and MDCK+shGRHL2/pGIPz cDNA was prepared as previously described (Chapter 2). The cDNA was amplified using 4ul of GoTaq Flexi buffer, $0.40 \mathrm{ul}$ of 10mM PCR nucleotides, $0.25 \mathrm{ul}$ of GoTaq DNA polymerase, 1 ul of template cDNA, 13.95ul of sterile water, and $0.20 \mathrm{ul}$ of forward and reverse primers. Primers were as follows: FGFR2-sp-F (6-FAM CGTACAAAGAACGGGGAGCA) and FGFR2-sp-R (GCTGAAGTCTGGCTTCTTGG). PCR products were digested with EcorV and Ava1. The AvaI site was present in FGFR2-IIIb but not FGFR2-IIIc. The fragments were run on a 4\% polyacrylamide gel with ROX as internal ladder. Quantification was performed comparing FGFR2-IIIc to FGFR2-IIIb bands.

\section{RET and GRHL2 ISH Staining and H\&E staining}

Kidneys were harvested from mice according to our WVU ACUC protocol. Kidneys were submerged in $10 \%$ formalin, paraffin embedded, and sectioned by the WVU Pathology Laboratory for Translational Medicine. To perform the section RNA in situs, ViewRNA ISH Tissue 2-plex 
assay (Affymetrix Panomics) was used. RNA probes for murine GRHL2 and RET were generated and used to evaluated their co-localization. The protocol was followed as described in user manual. Briefly, sections were deparaffinized, treated in pre-treatment solution, treated with proteinase K, hybridized for 3 hours in a humidified $40^{\circ}$ tissue culture incubator without $\mathrm{CO}_{2}$ with specific probes for mouse c-RET and GRHL2 designed by Panomic QuantiGENE. The bound probes were amplified using Pre-amp and Amp molecules then the labeled probes with fast red and fast blue substrate to produce red dots (RET) and green dots (GRHL2). Images were obtained using WVU MIF Olympus histology 20x microscope.

For H\&E sections, P30 GRHL2 ${ }^{+/+}$and GRHL2 ${ }^{+/}$kidneys were harvested, prepared, and H\&E stained by WVU Pathology Laboratory for Translational Medicine. Images were obtained using Olympus AX70 Provis microscope equipped with an Optronics MicroFire color CCD camera Using a 20x/0.70 UPlanApo objective.

\section{Ex Vivo Kidney Culture}

All experiments involving mice were approved by WVU IACUC. Hoxb7creEGFP transgenic mice were a gift from Carlton Bates (Children’s Hospital of Pittsburgh of UPMC, Pittsburgh, PA) and were described previously (Zhao et al., 2004). GRHL2 $2^{\text {flox/flox }}$ and GRHL2 $2^{+-}$ mice were a gift from Stephen Jane (Monash University), and of the C57BL6 inbred background. Mice of sexual maturity were timed bred to allow for embryo harvest at the specific developmental time points E13.5, E15.5, and E18.5. Coitus was verified by checking for vaginal plugs the following morning after timed mating. The embryos of pregnant mice were considered E0.5 at noon the day of vaginal plug detection. Embryo's kidneys were collected as described in Barak et al (Barak and Boyle, 2011). Briefly, a pregnant mouse was euthanized by cervical dislocation as approved in our IACUC protocol by highly trained laboratory personnel. 
Cervical dislocation was necessary to allow for harvest of kidneys before degradation of oxygen sensitive molecules occurred as well as to ensure survival of embryonic kidneys for ex vivo culturing. Next, the abdomen was washed with ethanol and an incision was made in the midline, cutting through the viscera and peritoneum. Subsequently, the uterine horn was dissected, and the embryos were extracted from the uterus. The embryos are separated from the yolk sac and placenta. The embryo's head was removed for PCR genotyping. An incision was made midline on the abdomen of the embryo, and the superficial organs were removed. The kidneys were removed and processed according the experiment being performed. Ex vivo kidney culture method was performed as previously described at indicated times (Zhao et al., 2004). For MMP inhibitor experiment, ex vivo embryonic kidney at E13.5 were cultured in 1uM batimastat (Tocris) or vector in culture media for 48 hours. Images were taken of GFP ureteric buds on Olympus MVX10 Macro Zoom, ORCA-Flash 4.0 Monochrome camera, Objective 2x, Zoom 4x, FITC, and using CellSen Imaging Software. Ureteric buds were counted for quantification. GRHL2 floxflox primary kidney cells and AdenoCre infection

Kidneys were harvested from GRHL2flox/flox mice as described above, digested with Collagenase/Dispase and then trypsin. Cells were plated on collagen 6 well dishes and allowed to grow for 48 hours. Cells were then stained for Dolichos Biflorus Agglutinin (DBA)-GFP (Vector Laboratories) to indicate collecting duct epithelial cells and flow sorted for GFP positivity. DBA-GFP positive and DBA-GFP negative cells plated on collagen coated 12 wells at 25,000 cells per well (Reichert et al., 2013). Ad5-CMV-CRE-eGFP (University of Iowa, Gene Transfer Vector core) was added at Multiplicity of Infection of 10 and 50 in DMEM+2\% FBS to each 12 well and protein lysates were made at 48 and 72 hours, and processed using previously described western blotting techniques 


\section{References}

An, J., and Rettig, M.B. (2005). Mechanism of von Hippel-Lindau Protein-Mediated Suppression of Nuclear Factor kappa B Activity. Mol. Cell. Biol. 25, 7546-7556.

Aue, A., Hinze, C., Walentin, K., Ruffert, J., Yurtdas, Y., Werth, M., Chen, W., Rabien, A., Kilic, E., Schulzke, J.-D., Schumann, M., and Schmidt-Ott, K.M. (2015). A Grainyhead-Like 2/Ovo-Like 2 Pathway Regulates Renal Epithelial Barrier Function and Lumen Expansion. J Am Soc Nephrol. 26, 2704-2715.

Banumathy, G., and Cairns, P. (2010). Signaling pathways in renal cell carcinoma. Cancer Biol. Therapy 10, 658-664.

Barak, H., and Boyle, S.C. (2011). Organ Culture and Immunostaining of Mouse Embryonic Kidneys. Cold Spring Harbor Protocols 2011, pdb.prot5558.

Barak, H., Huh, S.-H., Chen, S., Jeanpierre, C., Martinovic, J., Parisot, M., Bole-Feysot, C., Nitschké, P., Salomon, R., Antignac, C., Ornitz, David M., and Kopan, R. (2012). FGF9 and FGF20 Maintain the Stemness of Nephron Progenitors in Mice and Man. Dev. Cell 22, 11911207.

Basta, J.M., Robbins, L., Kiefer, S.M., Dorsett, D., and Rauchman, M. (2014). Sall1 balances self-renewal and differentiation of renal progenitor cells. Development 141, 1047-1058.

Boström, A.-K., Lindgren, D., Johansson, M.E., and Axelson, H. (2013). Effects of TGF- $\beta$ signaling in clear cell renal cell carcinoma cells. Biochem Biophys Res Commun. 435, 126-133.

Büttner, F., Winter, S., Rausch, S., Reustle, A., Kruck, S., Junker, K., Stenzl, A., Agaimy, A., Hartmann, A., Bedke, J., Schwab, M., and Schaeffeler, E. (2015). Survival Prediction of Clear Cell Renal Cell Carcinoma Based on Gene Expression Similarity to the Proximal Tubule of the Nephron. European Urology 68, 1016-1020.

Chacon-Heszele, M.F., Zuo, X., Hellman, N.E., McKenna, S., Choi, S.Y., Huang, L., Tobias, J.W., Park, K.M., and Lipschutz, J.H. (2014). Novel MAPK-dependent and -independent tubulogenes identified via microarray analysis of 3D-cultured Madin-Darby canine kidney cells. Am. J. Physio. - Renal Physiology 306, F1047-F1058. 
Cieply, B., Farris, J., Denvir, J., Ford, H.L., and Frisch, S.M. (2013). Epithelial-Mesenchymal Transition and Tumor Suppression Are Controlled by a Reciprocal Feedback Loop between ZEB1 and Grainyhead-like-2. Cancer Res 73, 6299-6309.

Cieply, B., Riley, P.t., Pifer, P.M., Widmeyer, J., Addison, J.B., Ivanov, A.V., Denvir, J., and Frisch, S.M. (2012). Suppression of the Epithelial-Mesenchymal Transition by Grainyhead-like2. Cancer Res 72, 2440-2453.

Clark, P.E. (2009). The role of VHL in clear-cell renal cell carcinoma and its relation to targeted therapy. Kidney international 76, 939-945.

Costantini, F. (2010). GDNF/Ret signaling and renal branching morphogenesis. Organogenesis 6, 252-262.

Faa, G., Gerosa, C., Fanni, D., Monga, G., Zaffanello, M., Van Eyken, P., and Fanos, V. (2012). Morphogenesis and molecular mechanisms involved in human kidney development. J. Cell. Physiol. 227, 1257-1268.

Farris, J.C., Pifer, P.M., Zheng, L., Gottlieb, E., Denvir, J., and Frisch, S.M. (2016). Grainyheadlike 2 Reverses the Metabolic Changes Induced by the Oncogenic Epithelial-mesenchymal Transition: Effects on Anoikis. Mol. Cancer Res. 14, 528-38

Frisch, S.M., and Francis, H. (1994). Disruption of epithelial cell-matrix interactions induces apoptosis. J Cell Biol 124, 619-626.

Gekle, M., Wünsch, S., Oberleithner, H., and Silbernagl, S. (1994). Characterization of two MDCK-cell subtypes as a model system to study principal cell and intercalated cell properties. Pflügers Archiv: European Journal Of Physiology 428, 157-162.

Hellman, N.E., Spector, J., Robinson, J., Zuo, X., Saunier, S., Antignac, C., Tobias, J.W., and Lipschutz, J.H. (2008). Matrix Metalloproteinase 13 (MMP13) and Tissue Inhibitor of Matrix Metalloproteinase 1 (TIMP1), Regulated by the MAPK Pathway, Are Both Necessary for Madin-Darby Canine Kidney Tubulogenesis. J Biol. Chem. 283, 4272-4282.

Ji, J., Li, Q., Xie, Y., Zhang, X., Cui, S., Shi, S., and Chen, X. (2012). Overexpression of Robo2 causes defects in the recruitment of metanephric mesenchymal cells and ureteric bud branching morphogenesis. Biochem Biophys Res Commun 421, 494-500. 
Jiang, Y., Zhang, W., Kondo, K., Klco, J.M., St. Martin, T.B., Dufault, M.R., Madden, S.L., Kaelin, W.G., and Nacht, M. (2003). Gene Expression Profiling in a Renal Cell Carcinoma Cell Line: Dissecting VHL and Hypoxia-Dependent Pathways. Mol. Cancer Res. 1, 453-462.

Kalluri, R., and Weinberg, R.A. (2009). The basics of epithelial-mesenchymal transition. J Clin Invest 119, 1420-1428.

Lee, S.J., Lattouf, J.-B., Xanthopoulos, J., Linehan, W.M., Bottaro, D.P., and Vasselli, J.R. (2008). Von Hippel-Lindau Tumor Suppressor Gene Loss in Renal Cell Carcinoma Promotes Oncogenic Epidermal Growth Factor Receptor Signaling via Akt-1 and MEK1. European urology 54, 845-853.

Li, Z., Fei, T., Zhang, J., Zhu, G., Wang, L., Lu, D., Chi, X., Teng, Y., Hou, N., Yang, X., Zhang, H., Han, J.-Dong J., and Chen, Y.-G. BMP4 Signaling Acts via Dual-Specificity Phosphatase 9 to Control ERK Activity in Mouse Embryonic Stem Cells. Cell Stem Cell 10, 171-182.

Majumdar, A., Vainio, S., Kispert, A., McMahon, J., and McMahon, A.P. (2003). Wnt11 and Ret/Gdnf pathways cooperate in regulating ureteric branching during metanephric kidney development. Development 130, 3175-3185.

Marom, K., Fainsod, A., and Steinbeisser, H. (1999). Patterning of the mesoderm involves several threshold responses to BMP-4 and Xwnt-8. Mechanisms of Development 87, 33-44.

Michos, O., Gonçalves, A., Lopez-Rios, J., Tiecke, E., Naillat, F., Beier, K., Galli, A., Vainio, S., and Zeller, R. (2007). Reduction of BMP4 activity by gremlin 1 enables ureteric bud outgrowth and GDNF/WNT11 feedback signalling during kidney branching morphogenesis. Development 134, 2397-2405.

Peruzzi, B., Athauda, G., and Bottaro, D.P. (2006). The von Hippel-Lindau tumor suppressor gene product represses oncogenic $\beta$-catenin signaling in renal carcinoma cells. Proc Natl Acad Sci USA 103, 14531-14536.

Piper, M., Georgas, K., Yamada, T., and Little, M. (2000). Expression of the vertebrate Slit Gene family and their putative receptors, the Robo genes, in the developing murine kidney. Mechanisms of Development 94, 213-217.

Reichert, M., Takano, S., Heeg, S., Bakir, B., Botta, G.P., and Rustgi, A.K. (2013). Isolation, culture and genetic manipulation of mouse pancreatic ductal cells. Nature protocols 8 , 13541365. 
Roca, H., Hernandez, J., Weidner, S., McEachin, R.C., Fuller, D., Sud, S., Schumann, T., Wilkinson, J.E., Zaslavsky, A., Li, H., Maher, C.A., Daignault-Newton, S., Healy, P.N., and Pienta, K.J. (2013). Transcription Factors OVOL1 and OVOL2 Induce the Mesenchymal to Epithelial Transition in Human Cancer. PLoS ONE 8, e76773.

Tang, M.-J., Worley, D., Sanicola, M., and Dressler, G.R. (1998). The RET-Glial Cell-derived Neurotrophic Factor (GDNF) Pathway Stimulates Migration and Chemoattraction of Epithelial Cells. J Cell Biol. 142, 1337-1345.

Tun, H.W., Marlow, L.A., von Roemeling, C.A., Cooper, S.J., Kreinest, P., Wu, K., Luxon, B.A., Sinha, M., Anastasiadis, P.Z., and Copland, J.A. (2010). Pathway Signature and Cellular Differentiation in Clear Cell Renal Cell Carcinoma. PLoS ONE 5, e10696.

Uehara, Y., and Kitamura, N. (1992). Expression of a human hepatocyte growth factor/scatter factor cDNA in MDCK epithelial cells influences cell morphology, motility, and anchorageindependent growth. J. Cell Biol. 117, 889-894.

Warzecha, C.C., Sato, T.K., Nabet, B., Hogenesch, J.B., and Carstens, R.P. (2009). ESRP1 and ESRP2 Are Epithelial Cell-Type-Specific Regulators of FGFR2 Splicing. Mol. Cell 33, 591-601.

Werth, M., Walentin, K., Aue, A., Schonheit, J., Wuebken, A., Pode-Shakked, N., Vilianovitch, L., Erdmann, B., Dekel, B., Bader, M., Barasch, J., Rosenbauer, F., Luft, F.C., and Schmidt-Ott, K.M. The transcription factor grainyhead-like 2 regulates the molecular composition of the epithelial apical junctional complex. Development 137, 3835-3845.

Werth, M., Walentin, K., Aue, A., Schönheit, J., Wuebken, A., Pode-Shakked, N., Vilianovitch, L., Erdmann, B., Dekel, B., Bader, M., Barasch, J., Rosenbauer, F., Luft, F.C., and Schmidt-Ott, K.M. (2010). The transcription factor grainyhead-like 2 regulates the molecular composition of the epithelial apical junctional complex. Development 137, 3835-3845.

Williams, R.D., Elliott, A.Y., Stein, N., and Fraley, E.E. (1978). In vitro cultivation of human renal cell cancer. II. Characterization of cell lines. In Vitro 14, 779-786.

Yu, J., Carroll, T.J., and McMahon, A.P. (2002). Sonic hedgehog regulates proliferation and differentiation of mesenchymal cells in the mouse metanephric kidney. Development 129, 53015312.

Zhao, H., Kegg, H., Grady, S., Truong, H.-T., Robinson, M.L., Baum, M., and Bates, C.M. (2004). Role of fibroblast growth factor receptors 1 and 2 in the ureteric bud. Dev. Biology 276, 403-415. 
Zhao, Q., Caballero, O.L., Davis, I.D., Jonasch, E., Tamboli, P., Yung, W.K.A., Weinstein, J.N., Kenna Shaw for the, T.r.n., Strausberg, R.L., and Yao, J. (2013). Tumor-Specific Isoform Switch of the Fibroblast Growth Factor Receptor 2 Underlies the Mesenchymal and Malignant Phenotypes of Clear Cell Renal Cell Carcinomas. Clin. Cancer Res. 19, 2460-2472. 


\section{Table 1. Gene regulated by HGF and GRHL2 via RNA-Seq}

Genes regulated by HGF in the absence of GRHL2

Gene ID Fold Change Significance in kidney development

(shGRHL2+hgf/shGRHL2)

\begin{tabular}{ccl}
\hline FGF9 & -23.6 & Maintains stemness of nephron progenitors (Barak et al., 2012) \\
\hline DUSP9 & -22.6 & Controls Erk activity in mouse embryonic stem cells (Li et al.) \\
SLIT2 & -9.8 & Restricts kidney induction to a single site (Ji et al., 2012) \\
Wnt11 & -7.6 & $\begin{array}{l}\text { Cooperates with GDNF/RET in regulating ureteric branching (Majumdar et } \\
\text { al., 2003) }\end{array}$ \\
\hline Wnt8a & 6.4 & Interacts with the BMP-4 pathway (Marom et al., 1999) \\
\hline
\end{tabular}




\section{Table 2. Gene regulated by HGF and GRHL2 via RNA-Seq}

Genes regulated by GRHL2

Gene ID Fold Change Significance in kidney development

(shGRHL2/GRHL2)

\begin{tabular}{|c|c|c|}
\hline MMP-13 & 15.1 & $\begin{array}{l}\text { Necessary for MDCK tubulogenesis } \\
\text { (Hellman et al., 2008) }\end{array}$ \\
\hline MMP-1 & 5.0 & $\begin{array}{l}\text { Necessary for MDCK tubulogenesis } \\
\text { (Chacon-Heszele et al., 2014) }\end{array}$ \\
\hline BMP-4 & 3.5 & $\begin{array}{l}\text { Inhibits ureteric branching morphogenesis } \\
\text { (Michos et al., 2007) }\end{array}$ \\
\hline TIMP1 & 3.3 & $\begin{array}{l}\text { Necessary for MDCK tubulogenesis } \\
\text { (Hellman et al., 2008) }\end{array}$ \\
\hline Robo1 & 2.1 & $\begin{array}{l}\text { Expressed in immature glomeruli (Piper et } \\
\text { al., 2000) }\end{array}$ \\
\hline FGFR2IIIC & 1.9 & $\begin{array}{l}\text { Predominate FGFR2 isoform in the } \\
\text { metanephric mesenchyme (Zhao et al., 2004) }\end{array}$ \\
\hline SALL1 & 1.7 & $\begin{array}{l}\text { Influences self-renewal and differentiation of } \\
\text { renal progenitor cells (Basta et al., 2014) }\end{array}$ \\
\hline GFR $\alpha$ & -2.7 & RET ligand-binding co-receptor ${ }^{\ddagger}$ (38) \\
\hline
\end{tabular}

Figure legends 
Figure 1. GRHL2 was downregulated in clear cell renal cell carcinoma. A. GRHL2, GATA3, and Snai1 mRNA expression from GEO website, GSE15641. B. Proposed mechanism of GRHL2 downregulation in ccRCC.

Figure 2. GRHL2 caused 786-0 ccRCC cells to undergo MET, prevented invasion, and increased anoikis. A. Western blot confirming GRHL2 expression in 786-0 cells. B. GRHL2 promoted an epithelial phenotype in 786-0 cells. C. GRHL2 suppressed HGF-induced invasion in 786-0 cell in three-dimensional matrigel culture. D. VHL and GRHL2 increased anoikis in 786-0 as measured by cellular DNA fragmentation assay. Values represent relative DNA fragmentation between samples.

Figure 3. GRHL2 is not expressed in normal proximal tubule human kidney cell lines. A. Quantitative PCR for GRHL2 mRNA in HMLE, HKC-8, and 786-0 cell lines. B. Cell line panel for GRHL2 protein across multiple cell lines. (See discussion in results). C. HKC-8 western band was resistant to GRHL2 shRNA knockdown via western blotting.

Figure 4. GRHL2 regulated FGFR2 isoform $3 b$ exon inclusion. MDCK parent, GRHL2, and shGRHL2 cDNAs were used to amplify FGFR2 3 splicing region. Differential digest was performed to determine the presence of FGFR2 3b (epithelial) and FGFR2 3c (mesenchymal) exon. Quantification was performed by comparing FGFR2 3c fragment to FGFR2 3b fragment

Figure 5. GRHL2 was down-regulated by HGF and GDNF. A. GDNF and HGF downregulated GRHL2. MDCK+RET cells were treated with vehicle, 60ng/ml HGF and/or 50ng/ml GDNF for 32 hours (Western blot). B. GDNF and HGF downregulated induction of GRHL2 promoters. MDCK+RET cells were cotransfected with GRHL2 promoter-luciferase reporter constructs. Values represent relative luciferase activity normalized to TK- $\beta$-galactosidase control. 
Figure 6. GRHL2 ${ }^{+/-}$mice showed lower number of glomeruli. A. Representative western blot of GRHL2 $2^{+/+}$and GRHL2 ${ }^{+/-}$kidney GRHL2 protein levels. B. Day $30 \mathrm{GRHL}^{+/+}$and $\mathrm{GRHL2}^{+/-}$kidney sections were compared via glomerulis $/ \mathrm{mm}^{2}(\mathrm{n}=5)$. GRHL2 ${ }^{+/+}$mice had a 9.7 \pm 2.6 per $\mathrm{mm}^{2}$ whereas GRHL $2^{+/-}$mice had $6.4 \pm 1.3$ per $\mathrm{mm}^{2}$. p value $<0.01$. C. Representative images of GRHL2 $2^{+/+}$and GRHL2 ${ }^{+/-}$glomeruli in H\&E sections imaged with 200.75 Fluar an Axiovert 200M microscope.

Figure 7. RET and GRHL2 co-localization in GRHL2 ${ }^{+/+}$and GRHL2 ${ }^{+/-}$mice. Representative images of RET (Red), GRHL2 (Green), and merged co-localization using a ViewRNA ISH Tissue 2-plex assay system. Images were obtained using Zeiss Violet Confocal, 20x.

Figure 8. Effect of ureteric epithelial specific GRHL2 ${ }^{-/-}$on ureteric tip count. A. Control and GRHL2 $2^{\text {UB-/- }}$ kidneys were harvested at E13.5 and cultured for 48 hours. B. Control and GRHL2 $2^{\text {UB-/- }}$ kidneys were harvested at E18.5. Both A,B ureteric tips were counted and normalized to area $\left(\mathrm{mm}^{2}\right)$. Images were obtained using Olympus MVX10 Macro Zoom, ORCAFlash 4.0 Monochrome camera, Objective 2x, Zoom 4x, FITC, and using CellSen Imaging Software.

Figure 9. GRHL2 ${ }^{\text {flox/flox }}$ mice generated a $57 \mathrm{kDA}$ active GRHL2 isoform. A. Schematic of Jane GRHL2 $2^{\text {flox/flox }}$ mouse model. B. Predicted GRHL2 isoform size depending on ATG Start Codon. C. GRHL2 $2^{\text {flox/flox }}$ primary kidney cells treated with adenoviral-Cre. DBA is an epithelial marker used for flow sorting of primary kidney cells. 
Figure 1

A.

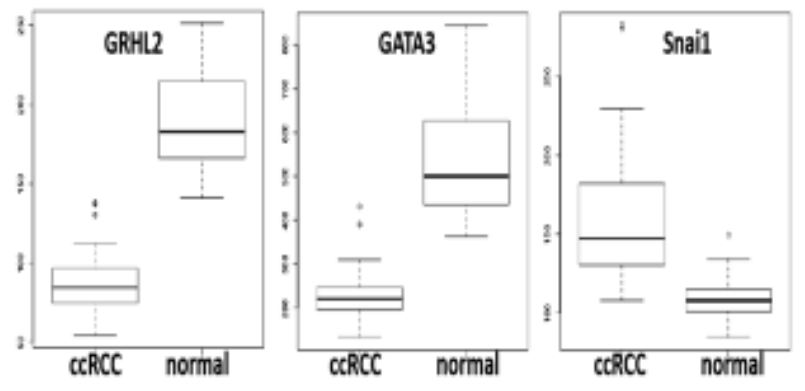

B.

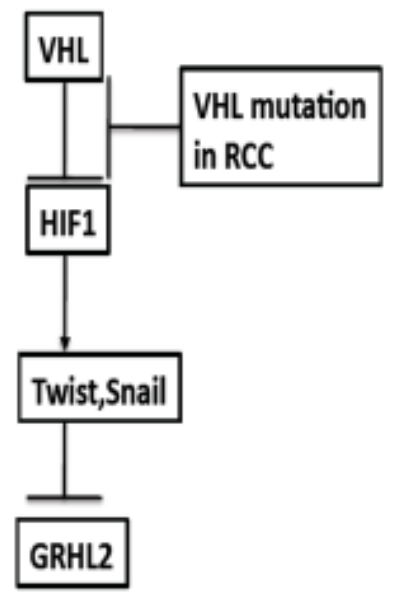


Figure 2

A.

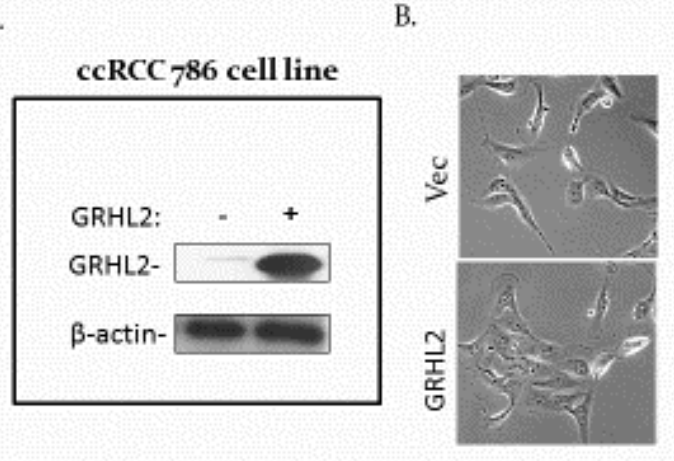

c.

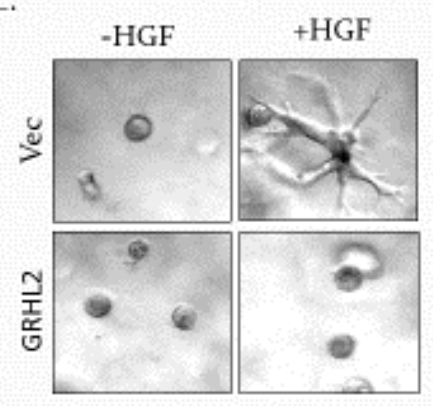

D.

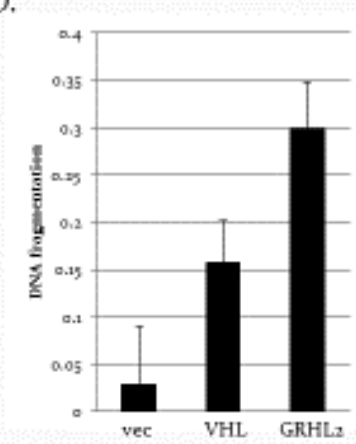


Figure 3

A.

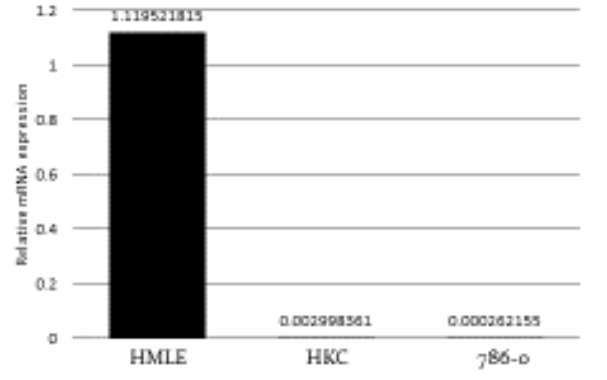

B.

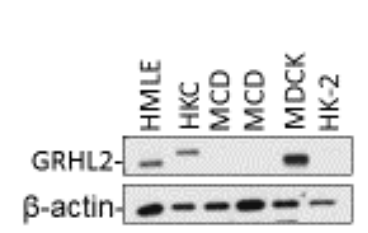

c.

GRHL2 shRNA 1223
GRHL2 $70 \mathrm{kD}$
GRHL2 $50 \mathrm{kD}$
B-actin


Figure 4

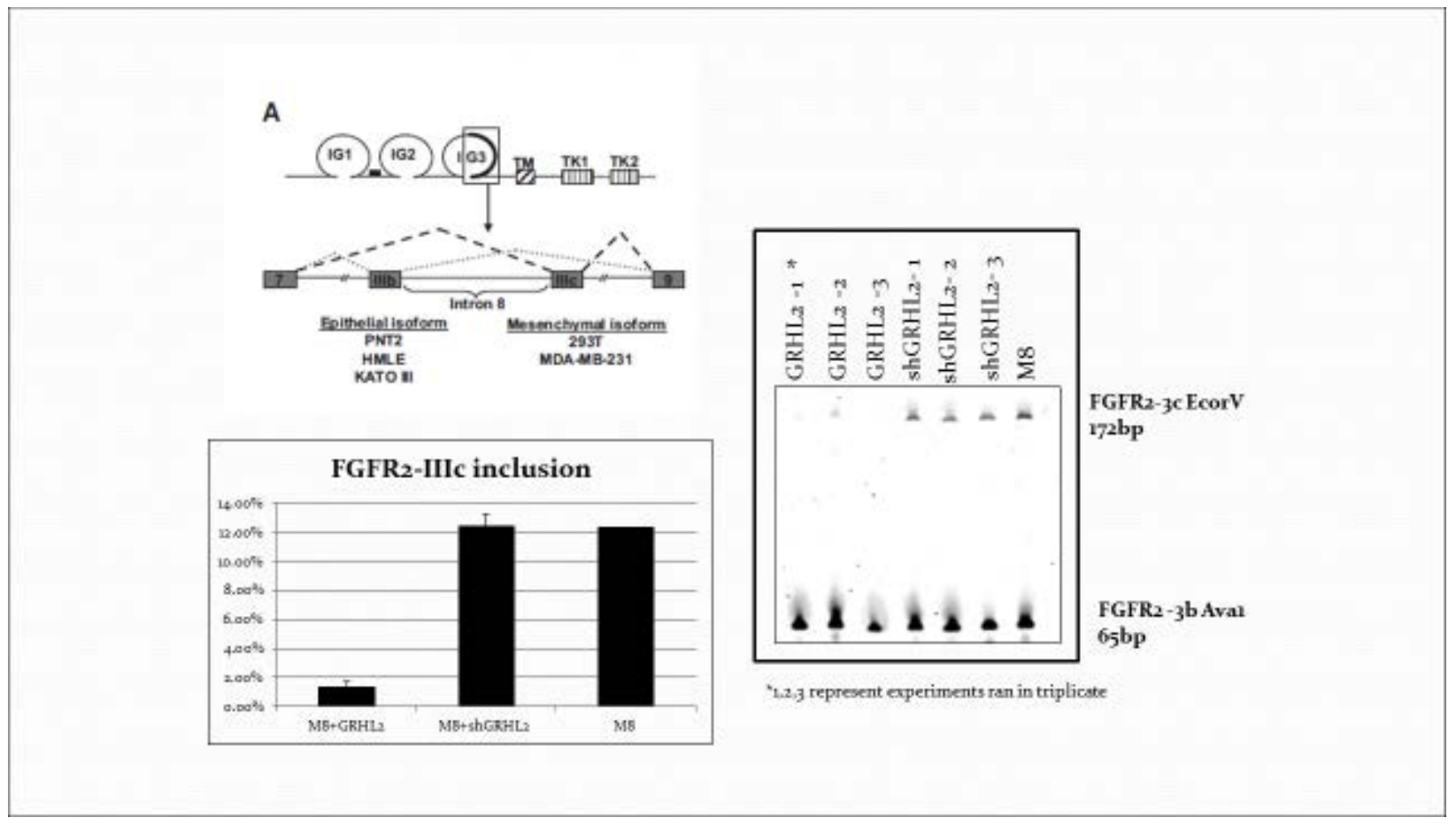


Figure 5

A.

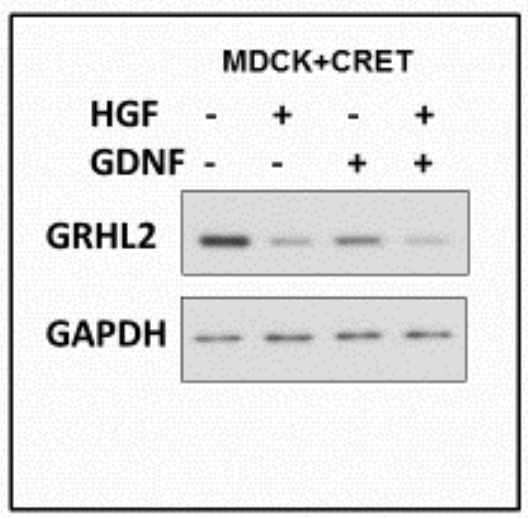

B.

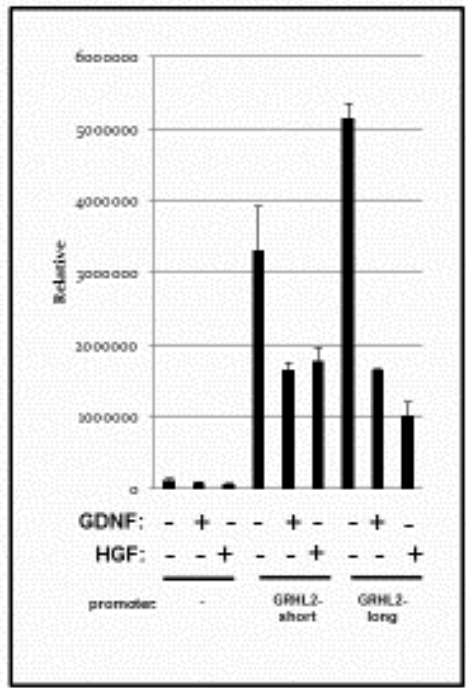


Figure 6

Figure 6

A.

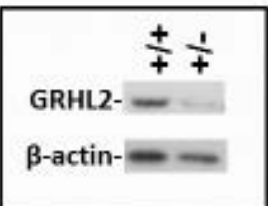

B.

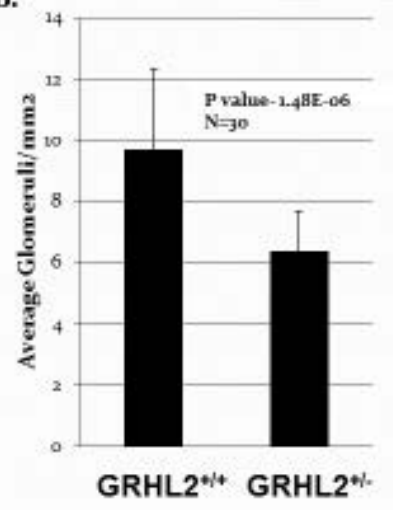

c.
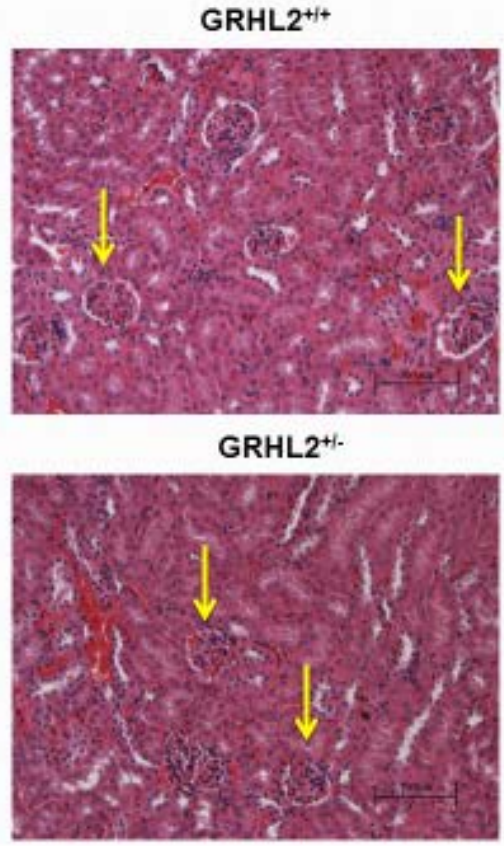
Figure 7

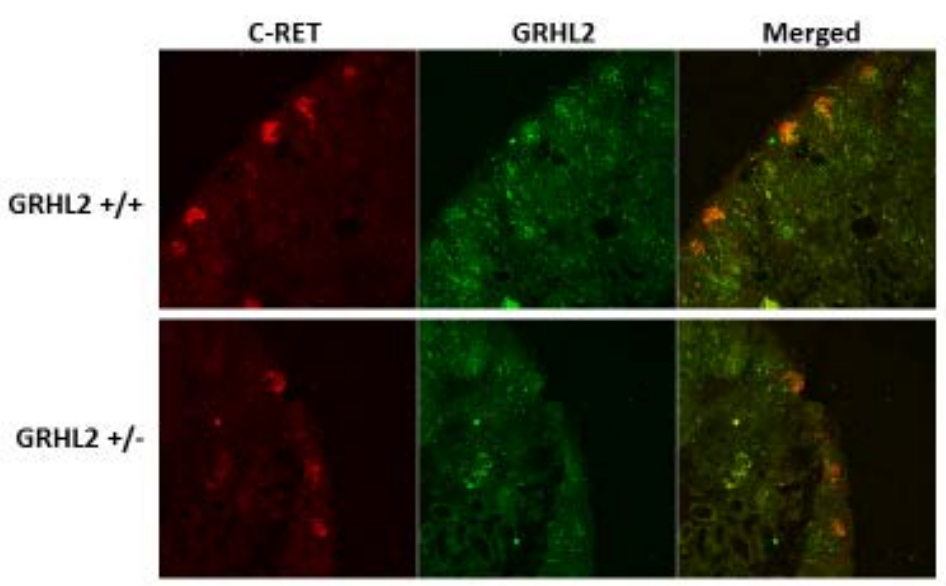


Figure 8

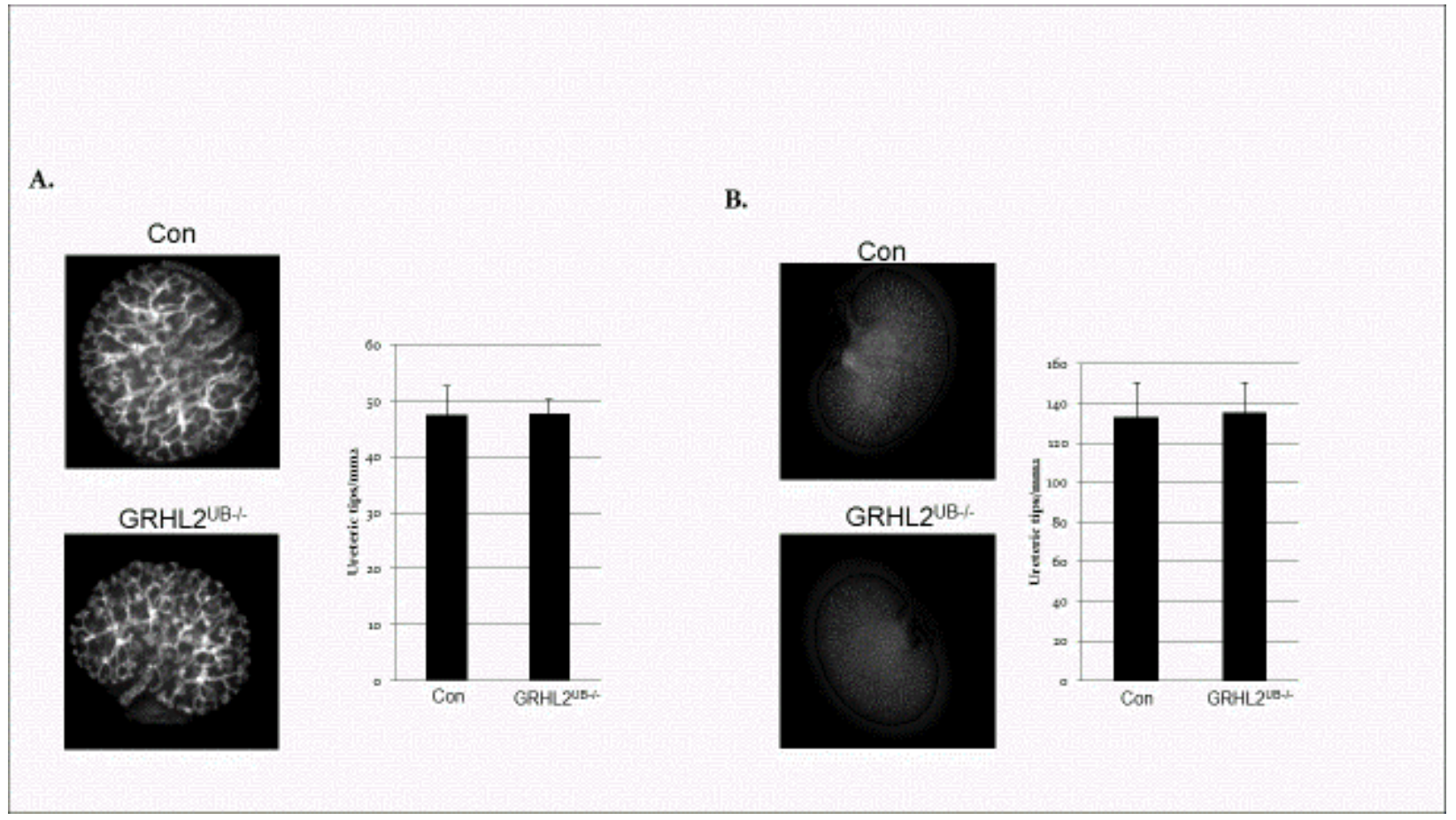




\section{Figure 9}

A.

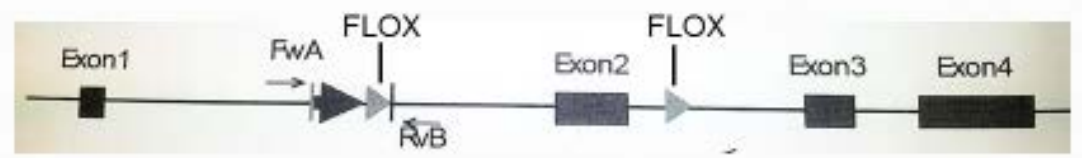

B.

\begin{tabular}{|l|l|l|}
\hline $\begin{array}{l}\text { ATG } \\
\text { Codon } \\
\text { Start }\end{array}$ & $\begin{array}{l}\text { Predicted } \\
\text { amino acids }\end{array}$ & $\begin{array}{l}\text { Predicted } \\
\text { kDa }\end{array}$ \\
\hline Exon 1 & 625 aа & $72 \mathrm{kDa}$ \\
\hline Exon 4 & 495 aа & $57 \mathrm{kDa}$ \\
\hline
\end{tabular}

GRHL2 flox/flox kidney primary cells

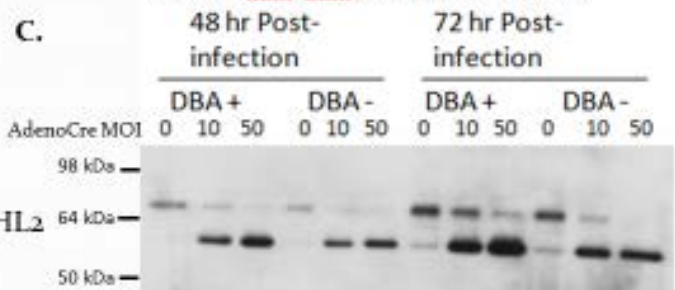


Chapter 4

Conclusion and Future Directions 


\section{Conclusions}

The present work examined the role of GRHL2 in an array of different systems and circumstances. GRHL2 was not expressed in the normal kidney proximal tubule, and therefore highly unlikely to regulate clear cell renal cell carcinoma tumor progression. However, GRHL2 could be used as a biomarker to distinguish between different renal cell carcinoma subtypes. GRHL2 was involved in multiple signaling pathways and processes that are crucial in kidney development, but further investigation using a ureteric epithelium specific GRHL $2^{\text {flox/flox }}$ mouse is required to understand the role of GRHL2 in the developing kidney.

In the MDCK model, GRHL2 was critical for cystogenesis but suppressed tubulogenesis. For the first time, GRHL2 was shown to interact with the histone acetyltransferase co-activator protein, p300, and inhibit its HAT activity thereby preventing its transcriptional activation of target genes. These findings place GRHL2 among a rare group of proteins that can inhibit p300 HAT activity. The p300 HAT inhibitory domain of GRHL2 was mapped to amino acids 425437 with the following sequence: IRDEERKQNRKKG. These 13 amino acids were required for GRHL2 to suppress tubulogenesis and cause a mesenchymal-epithelial transition. Since p300 is critical for cells to differentiate, GRHL2's inhibition of p300 HAT activity provides a mechanism for GRHL2 to enforce the default epithelial phenotype. This work mechanistically positions GRHL2, a master regulator of the default epithelial phenotype, to antagonize p300, a

co-activator required for cell differentiation, having vast implications in both developmental and cancer contexts. 


\section{Future Directions}

Because the ureteric epithelium GRHL2 $2^{\text {flox/flox }}$ model our laboratory generated was not informative, there are many unanswered questions left about GRHL2 and kidney development. Previous results from our laboratory and from Schmidt-Ott et. al. show that GRHL2 depletion in global GRHL2 ${ }^{-/-}$mice, miMCD cells, and MDCK cells affect kidney tubulogenesis and lumen size, highly suggestive of an important role in kidney development (Werth et al., 2010; Aue et al., 2015). The GRHL2 $2^{\text {flox/flox }}$ mice generated by Aue et. al. (which deletes exon 4) could be used in cooperation with the Zhao et. al. HOXB7creEGFP mice to elucidate the role of GRHL2 in kidney development (Zhao et al., 2004; Walentin et al., 2015). The expected outcome of these experiments would be renal agenesis because of the role of GRHL2 in establishing epithelial phenotype and cell polarity. GRHL2 would be a tool for examining the importance of the ureteric bud in kidney development. From a clinical perspective, GRHL2 mutations could be examined for causing renal agenesis or renal duplication anomalies.

As one of the main focuses of our laboratory is breast cancer, it would be highly informative to examine the effects of GRHL2 on mammary development. OVOL2, a GRHL2 regulated epithelial transcription factor, is known to affect mammary morphogenesis. When OVOL2 is depleted in the mammary glands, the cells undergo an EMT and decrease milk production and alveologenesis (Roca et al., 2013). At the very least, GRHL2 would be expected to have a similar effect as OVOL2 on mammary development. The loss of GRHL2 in these cells would most likely cause a mesenchymal phenotype. Also, GRHL2 may cause a more severe phenotype than OVOL2 since it regulates a vast number of genes other than OVOL2 in RNASeq (Farris et al., 2016). If the GRHL2 phenotype was more severe, a rescue of OVOL2 would be highly informative demonstrating alternative pathways not regulated through the 
GRHL2/OVOL2 axis. These results would significantly expand the understanding of GRHL2 in organogenesis. This examination might provide novel GRHL2 interaction partners that occur during the developmental process with potential influences on breast cancer progression.

Our laboratory proposed the default epithelial phenotype hypothesis for both development and cancer differentiation. Under this hypothesis, the epithelial phenotype is adopted automatically by a cell in the absence of genomic/epigenetic alterations or inductive differentiation signaling (Frisch, 1997). Consistent with this idea, E-cadherin-expressing cells are the first to emerge at the late two cell stage; these eventually give rise to all differentiated cell types of the adult organism (Hyafil et al., 1980; Vestweber and Kemler, 1985; Fleming et al., 1989; Larue et al., 1994). Furthermore, GRH, the Drosophila GRHL2 homolog, is one of the first proteins expressed in the pre-cellular blastoderm zygote. GRH is responsible for the regulation of the maternal-to-zygotic transition, the process where a zygote degrades maternal mRNA and starts transcribing its own mRNA (Harrison et al., 2010).

In recent studies, other laboratories have suggested the default epithelial phenotype hypothesis may explain the mesenchymal-epithelial transition (MET) that occurs at metastatic sites (Thiery and Sleeman, 2006; Polyak and Weinberg, 2009; Tam and Weinberg, 2013). Our results demonstrating the interaction between p300 and GRHL2 provide another piece of support for this hypothesis. The critical role of p300 in the differentiation of muscle, erythroid lineages, osteoblasts, oligodendrocytes, and stem cells has been clearly established. The role of p300 in differentiation demonstrates the importance of epigenetic modifications in this process (Puri et al., 1997; Oike et al., 1999; Blobel, 2000; Polesskaya et al., 2001; Narayanan et al., 2004; Teo and Kahn, 2010; Zhang et al., 2016). The ability of GRHL2 to inhibit p300 can explain the default epithelial phenotype hypothesis since critical co-activators such as p300 are required for 
differentiation-specific but not epithelial-specific gene expression (Frisch, 1997). These findings could also extend to critical roles in EMT, in contexts such as tubulogenesis and oncogenesis. In summary, p300 and related co-activators are crucial for cells to differentiate. Under the defaultphenotype hypothesis, the inhibition of p300 by GRHL2 provides a mechanism for GRHL2 to enforce the default epithelial phenotype.

As described in Chapter 1, Roeder et. al. demonstrated that p300 interacts with the SET1 complex through a p53 mediated event to regulate H3K4 trimethylation at activated promoters (Tang et al., 2013). The SET1/MLL histone methyltransferase family is comprised of SET1A, SET1B, SET1C MLL1, MLL2, MLL3 and MLL4 (Yokoyama et al., 2004; Dou et al., 2005; Cho et al., 2007; Wu et al., 2008). MLL1 has also been shown to interact with CBP (Ernst et al., 2001). Also noted in Chapter 1, EMT is highly dependent on epigenetic regulation. In our laboratory, GRHL2 was found to interact with MLL3/4 by a yeast two hybrid screen, and these results were confirmed by MLL3/GRHL2 co-immunoprecipitation (data not shown). These findings have interesting implications. GRHL2 interacts with two histone modifying factors, p300 and MLL3/4 which collaborate for gene activation (Tang et al., 2013). Our laboratory has formed a collaboration with Robert Roeder (Rockefeller University) to examine these interactions. Theoretically, GRHL2 could be recruiting these proteins to specific promoters, inhibiting the histone modifying factors, and/or modulating their enzymatic activities. This would link GRHL2 directly to epigenetic methylation and acetylation events, and provide another mechanism of how GRHL2 establishes and maintains the epithelial phenotype.

The result of our research with the most potential impact is the inhibition of p300 HAT domain by GRHL2. p300 HAT inhibitors are currently an intense area of research with implications in cancer, diabetes, HIV infections, heart disease, and platelet cell production 
(Chapter 1) (Dancy and Cole, 2015). p300 HAT inhibitors have not been investigated in clinical trials to date (Dancy and Cole, 2015). Two of the major issues facing p300 HAT inhibitors are lack of specificity when compared to other histone acetyltransferase families and/or cell impermeability making the chemicals difficult to use in vivo conditions. GRHL2 appears to potently inhibit the p300 HAT domain, but more kinetic experiments need to be completed to determine the half maximal inhibitory concentration. The GRHL2 13aa peptide could be targeted for cellular uptake by attaching the peptide to a peptide/protein transduction domain (Lönn and Dowdy, 2015), and its effect on cellular acetylation would be examined.

Furthermore, the GRHL2 peptide could be mutated to find the critical residues for p300 inhibition. These critical residues could be used to model the GRHL2 and p300 interaction providing more information about the HAT domain and targetable sites that may have previously gone undiscovered. The ability of GRHL2 to inhibit CBP or other histone acetyltransferase families would be informative and demonstrate the generalizability of GRHL2's HAT inhibition.

Our laboratory has previously published that activation of both the TGF- $\beta$ and Wnt pathways are able to downregulate GRHL2 (Cieply et al., 2012), and our present work has demonstrated that HGF pathway is able to downregulate GRHL2. All of these pathways are involved in tumor progression, and clinical trials are underway with selective inhibitors of these pathways in different cancers (Blagodatski et al., 2014; Neuzillet et al., 2015; Zhang et al., 2015). The examination of these inhibitors in the context of GRHL2 could provide a mechanism to pharmacologically upregulate GRHL2 and maintain the epithelial phenotype thereby preventing cancer metastasis.

In summary, GRHL2 is critical for establishing the epithelial phenotype, and more research is required to understand its complex biological role. 


\section{References}

Aue, A., Hinze, C., Walentin, K., Ruffert, J., Yurtdas, Y., Werth, M., Chen, W., Rabien, A., Kilic, E., Schulzke, J.-D., Schumann, M., and Schmidt-Ott, K.M. (2015). A Grainyhead-Like 2/Ovo-Like 2 Pathway Regulates Renal Epithelial Barrier Function and Lumen Expansion. J Am Soc Nephrol. 26, 2704-2715.

Blagodatski, A., Poteryaev, D., and Katanaev, V.L. (2014). Targeting the Wnt pathways for therapies. Mol. Cell. Therapies 2, 1-15.

Blobel, G.A. (2000). CREB-binding protein and p300: molecular integrators of hematopoietic transcription. Blood 95, 745-755.

Cho, Y.-W., Hong, T., Hong, S., Guo, H., Yu, H., Kim, D., Guszczynski, T., Dressler, G.R., Copeland, T.D., Kalkum, M., and Ge, K. (2007). PTIP Associates with MLL3- and MLL4containing Histone H3 Lysine 4 Methyltransferase Complex. J. Biol. Chem. 282, 20395-20406.

Cieply, B., Riley, P.t., Pifer, P.M., Widmeyer, J., Addison, J.B., Ivanov, A.V., Denvir, J., and Frisch, S.M. (2012). Suppression of the Epithelial-Mesenchymal Transition by Grainyhead-like2. Cancer Res 72, 2440-2453.

Dancy, B.M., and Cole, P.A. (2015). Protein lysine acetylation by p300/CBP. Chemical Reviews 115, 2419-2452.

Dou, Y., Milne, T.A., Tackett, A.J., Smith, E.R., Fukuda, A., Wysocka, J., Allis, C.D., Chait, B.T., Hess, J.L., and Roeder, R.G. (2005). Physical Association and Coordinate Function of the H3 K4 Methyltransferase MLL1 and the H4 K16 Acetyltransferase MOF. Cell 121, 873-885.

Ernst, P., Wang, J., Huang, M., Goodman, R.H., and Korsmeyer, S.J. (2001). MLL and CREB Bind Cooperatively to the Nuclear Coactivator CREB-Binding Protein. Mol. Cell. Biol. 21, 2249-2258.

Farris, J.C., Pifer, P.M., Zheng, L., Gottlieb, E., Denvir, J., and Frisch, S.M. (2016). Grainyheadlike 2 Reverses the Metabolic Changes Induced by the Oncogenic Epithelial-mesenchymal Transition: Effects on Anoikis. Mol. Cancer Res.

Fleming, T.P., McConnell, J., Johnson, M.H., and Stevenson, B.R. (1989). Development of tight junctions de novo in the mouse early embryo: control of assembly of the tight junction-specific protein, ZO-1. J. Cell Biol. 108, 1407-1418. 
Frisch, S.M. (1997). The epithelial cell default-phenotype hypothesis and its implications for cancer. Bioessays 19, 705-709.

Harrison, M.M., Botchan, M.R., and Cline, T.W. (2010). Grainyhead and Zelda compete for binding to the promoters of the earliest-expressed Drosophila genes. Dev Biol 345, 248-255.

Hyafil, F., Morello, D., Babinet, C., and Jacob, F. (1980). A cell surface glycoprotein involved in the compaction of embryonal carcinoma cells and cleavage stage embryos. Cell 21, 927-934.

Larue, L., Ohsugi, M., Hirchenhain, J., and Kemler, R. (1994). E-cadherin null mutant embryos fail to form a trophectoderm epithelium. Proc Natl Acad Sci USA 91, 8263-8267.

Lönn, P., and Dowdy, S.F. (2015). Cationic PTD/CPP-mediated macromolecular delivery: charging into the cell. Expert Opin Drug Deliv. 12, 1627-1636.

Narayanan, K., Srinivas, R., Peterson, M.C., Ramachandran, A., Hao, J., Thimmapaya, B., Scherer, P.E., and George, A. (2004). Transcriptional Regulation of Dentin Matrix Protein 1 by JunB and p300 during Osteoblast Differentiation. J. Biol. Chem. 279, 44294-44302.

Neuzillet, C., Tijeras-Raballand, A., Cohen, R., Cros, J., Faivre, S., Raymond, E., and de Gramont, A. (2015). Targeting the TGF $\beta$ pathway for cancer therapy. Pharmacol. Ther. 147, 2231.

Oike, Y., Takakura, N., Hata, A., Kaname, T., Akizuki, M., Yamaguchi, Y., Yasue, H., Araki, K., Yamamura, K.-i., and Suda, T. (1999). Mice Homozygous for a Truncated Form of CREBBinding Protein Exhibit Defects in Hematopoiesis and Vasculo-angiogenesis. Blood 93, 27712779.

Polesskaya, A., Naguibneva, I., Fritsch, L., Duquet, A., Ait-Si-Ali, S., Robin, P., Vervisch, A., Pritchard, L.L., Cole, P., and Harel-Bellan, A. (2001). CBP/p300 and muscle differentiation: no HAT, no muscle. EMBO J. 20, 6816-6825.

Polyak, K., and Weinberg, R.A. (2009). Transitions between epithelial and mesenchymal states: acquisition of malignant and stem cell traits. Nat Rev Cancer 9, 265-273.

Puri, P.L., Sartorelli, V., Yang, X.-J., Hamamori, Y., Ogryzko, V.V., Howard, B.H., Kedes, L., Wang, J.Y.J., Graessmann, A., Nakatani, Y., and Levrero, M. (1997). Differential Roles of p300 and PCAF Acetyltransferases in Muscle Differentiation. Mol. Cell 1, 35-45. 
Roca, H., Hernandez, J., Weidner, S., McEachin, R.C., Fuller, D., Sud, S., Schumann, T., Wilkinson, J.E., Zaslavsky, A., Li, H., Maher, C.A., Daignault-Newton, S., Healy, P.N., and Pienta, K.J. (2013). Transcription Factors OVOL1 and OVOL2 Induce the Mesenchymal to Epithelial Transition in Human Cancer. PLoS ONE 8, e76773.

Tam, W.L., and Weinberg, R.A. (2013). The epigenetics of epithelial-mesenchymal plasticity in cancer. Nat Med 19, 1438-1449.

Tang, Z., Chen, W.-Y., Shimada, M., Nguyen, U.T.T., Kim, J., Sun, X.-J., Sengoku, T., McGinty, R.K., Fernandez, J.P., Muir, T.W., and Roeder, R.G. (2013). SET1 and p300 Act Synergistically, through Coupled Histone Modifications, in Transcriptional Activation by p53. Cell 154, 297-310.

Teo, J.-L., and Kahn, M. (2010). The Wnt signaling pathway in cellular proliferation and differentiation: A tale of two coactivators. Adv. Drug Deliv. Rev. 62, 1149-1155.

Thiery, J.P., and Sleeman, J.P. (2006). Complex networks orchestrate epithelial-mesenchymal transitions. Nat Rev Mol Cell Biol 7, 131-142.

Vestweber, D., and Kemler, R. (1985). Identification of a putative cell adhesion domain of uvomorulin. EMBO J. 4, 3393-3398.

Walentin, K., Hinze, C., Werth, M., Haase, N., Varma, S., Morell, R., Aue, A., Pötschke, E., Warburton, D., Qiu, A., Barasch, J., Purfürst, B., Dieterich, C., Popova, E., Bader, M., Dechend, R., Staff, A.C., Yurtdas, Z.Y., Kilic, E., and Schmidt-Ott, K.M. (2015). A Grhl2-dependent gene network controls trophoblast branching morphogenesis. Development 142, 1125-1136.

Werth, M., Walentin, K., Aue, A., Schönheit, J., Wuebken, A., Pode-Shakked, N., Vilianovitch, L., Erdmann, B., Dekel, B., Bader, M., Barasch, J., Rosenbauer, F., Luft, F.C., and Schmidt-Ott, K.M. (2010). The transcription factor grainyhead-like 2 regulates the molecular composition of the epithelial apical junctional complex. Development 137, 3835-3845.

Wu, M., Wang, P.F., Lee, J.S., Martin-Brown, S., Florens, L., Washburn, M., and Shilatifard, A. (2008). Molecular Regulation of H3K4 Trimethylation by Wdr82, a Component of Human Set1/COMPASS. Mol. Cell. Biol. 28, 7337-7344.

Yokoyama, A., Wang, Z., Wysocka, J., Sanyal, M., Aufiero, D.J., Kitabayashi, I., Herr, W., and Cleary, M.L. (2004). Leukemia Proto-Oncoprotein MLL Forms a SET1-Like Histone Methyltransferase Complex with Menin To Regulate Hox Gene Expression. Mol. Cell. Biol. 24, 5639-5649. 
Zhang, L., He, X., Liu, L., Jiang, M., Zhao, C., Wang, H., He, D., Zheng, T., Zhou, X., Hassan, A., Ma, Z., Xin, M., Sun, Z., Lazar, Mitchell A., Goldman, Steven A., Olson, Eric N., and Lu, Q.R. (2016). Hdac3 Interaction with p300 Histone Acetyltransferase Regulates the Oligodendrocyte and Astrocyte Lineage Fate Switch. Dev. Cell 36, 316-330.

Zhang, Y., Jain, R., and Zhu, M. (2015). Recent Progress and Advances in HGF/MET-Targeted Therapeutic Agents for Cancer Treatment. Biomedicines 3, 149.

Zhao, H., Kegg, H., Grady, S., Truong, H.-T., Robinson, M.L., Baum, M., and Bates, C.M. (2004). Role of fibroblast growth factor receptors 1 and 2 in the ureteric bud. Dev. Biology 276, 403-415. 
Chapter 5

\section{Curriculum Vitae}




\section{Phillip M. Pifer}

22 REGENCY CT, MORGANTOWN, WV 26505

PHONE: (304) 834-6143

EMAIL: PPIFER@MIX.WVU.EDU

\section{EDUCATION}

Ph.D. M.D.-Ph.D. Dual degree scholar: Aug 2010-May 2018

Anticipated degree date:

Ph.D.: May 2018

Time in Ph.D.: Aug 2010June 2016

\author{
West Virginia University, Morgantown, WV \\ Department of Biochemistry, \\ Program in Cancer Cell Biology, \\ School of Medicine
}

Dissertation Title: Grainyhead-like-2 inhibits the coactivator p300, suppressing tubulogenesis and the epithelial-mesenchymal transition.

Dissertation Advisor: Dr. Steven M. Frisch, Ph.D.

Dissertation Defense Date: 06/24/2016
M.D. M.D.-Ph.D. Dual degree scholar: Aug 2010- May 2018

Anticipated degree date:

M.D.: May 2018

B.S. Chemistry: Aug 2006- May 2010

Honors: Summa Cum Laude
West Virginia University, Morgantown, WV

Department of Chemistry,

Eberly School of Arts \& Sciences

\section{RESEARCH EXPERIECNES}

MD/PhD Trainee, Dept. of Biochemistry, WVU

2011 - Present

- Advisor: Steven M. Frisch, PhD. “Grainyhead-like-2 inhibits the coactivator p300, suppressing tubulogenesis and the epithelial-mesenchymal transition"

Visiting Graduate Researcher, Children's Hospital of Pittsburgh, Dept. of Nephrology

- Advisor: Carlton M. Bates, MD. Laboratory methods of the developing kidney.

Undergraduate Researcher, Dept. of Chemistry, WVU

- Advisor: Justin Legleiter, PhD. "Point mutations in A $\beta$ result in the formation of distinct polymorphic aggregates in the presence of lipid bilayers." 
Pifer, P. M., Farris, J. C., Thomas, A. L., Stoilov, P., Denvir, J., Smith, D. M., \& Frisch, S. M. (2016). Grainyheadlike-2 inhibits the coactivator p300, suppressing tubulogenesis and the epithelial-mesenchymal transition. Molecular Biology of the Cell.

Farris, J. C., Pifer, P. M., Zheng, L., Gottlieb, E., Denvir, J., \& Frisch, S. M. (2016). Grainyhead-like 2 Reverses the Metabolic Changes Induced by the Oncogenic Epithelial-mesenchymal Transition: Effects on Anoikis. Molecular Cancer Research.

Cieply, B., Riley, P. t., Pifer, P. M., Widmeyer, J., Addison, J. B., Ivanov, A. V., . . Frisch, S. M. (2012). Suppression of the Epithelial-Mesenchymal Transition by Grainyhead-like-2. Cancer Res, 72(9), 24402453.

Pifer, P. M., Yates, E. A., \& Legleiter, J. (2011). Point mutations in A $\beta$ result in the formation of distinct polymorphic aggregates in the presence of lipid bilayers. PLoS One, 6(1).

Kumar, B., Pifer, P. M., Giovengo, A., \& Legleiter, J. (2010). The effect of set point ratio and surface Young's modulus on maximum tapping forces in fluid tapping mode atomic force microscopy. Journal of Applied Physics, 107(4), 044508.

\section{TECHNIQUES}

Cell and Molecular Biology: Tissue cell culture, three dimensional cell culture, PCR Primer Design, Molecular Cloning Techniques (Including sub-cloning, site-directed mutagenesis, and plasmid preparation), RNA isolation (from primary tissues and cells), DNA purification, qRT-PCR, RNA-Seq preparation, cell transfection, mammalian cell retroviral and lentiviral transduction, luciferase reporter assays, immunoblotting (western blot), immunofluorescent staining, histone acetylation assays, bacterial and mammalian protein purification, Ingenuity Pathway Analysis examination, immunoprecipitation, chromatin immunoprecipitation, Anoikis assays (caspase 3/7 activation assays, cell death ELISA, and cell viability assays), and confocal microscopy for MDCK Cysts.

Animal and Kidney models: Timed breeding, embryonic kidney harvesting, ex vivo kidney culture, kidney imaging, colony maintenance, mouse genotyping, folic acid kidney injury model, murine models of mammary development, tumor burden scoring of mice, H\&E Staining, and RNA in situ hybridization assays.

\section{ABSTRACTS, PRESENTATIONS, and CONFERENCES}

\section{$\underline{\text { Abstracts }}$}

Pifer, P.M., Cieply, B., Frisch, S.M. The role of GRHL-1 and GRHL-2 in clear cell renal cell carcinoma. - Van Liere Research Convocation- West Virginia University, Morgantown, WV (2013)

Pifer, P.M., and Legleiter, J. The effect of mutant A-beta amyloid peptide aggregation on the stability of model lipid bilayers. - 54th Annual meeting of Biophysical Society February 20-24 (2010); WVNANO Initiative Research Symposium (2009); WVU American Chemical Society Student Affiliates Chemical Symposium (2009)

\section{$\underline{\text { Presentations }}$}

Pifer, PM., Farris, JC. The Physician-Scientist career track: How to be a successful MD-PhD candidate- (May 31st, 2016) - WVU Cancer Institute undergraduate research program invited speaker 
Pifer, PM. Advancements in Melanoma- PD1 inhibitors. (Jan. 21'st, 2016) - WVU Cancer Cell Biology Journal Club

Pifer, PM. Role of grainyhead-like 2 in the establishment of the epithelial phenotype (Jun. 24st, 2015) - WVU Genomics Group invited speaker

Pifer, PM. The MD-PhD Track: A Brief Overview. (Jun. 8st, 2015) - IDeA Network of Biomedical Research Excellence (INBRE) invited speaker

Pifer, PM, Frisch SM. GRHL2 in Kidney Tubulogenesis. (Nov. 16, 2014) Department of Biochemistry- Cancer Cell Biology Seminar.

Pifer, PM, Frisch SM. GRHL2 in Kidney Tubulogenesis. (Apr. 28, 2014) Department of Biochemistry - Cancer Cell Biology Dissertation Proposal.

Pifer, PM. Sharma SB, Farrugia MK. The MD-PhD Track: A Brief Overview. (Feb. 27st, 2014) - WVU American Chemical Society Student Affiliates invited speaker

Pifer, PM, Frisch SM. The role of GRHL1 and GRHL2 in clear cell renal cell carcinoma. (Jan. 30, 2013) Department of Biochemistry - Cancer Cell Biology Seminar.

Pifer, PM, Sharma SB, Farrugia MK, Pilkerton CK, Turner RC. The Physician-Scientist career track: How to be a successful MD-PhD candidate - (2011) WVU chapter of the American Physician-Scientist Association (APSA) sponsored talk

\section{Conferences}

American Physician Scientist Association Regional Meeting, (Oct 2015) SUNY UPSTATE Medical University, Syracuse, NY,

Tackling Translational Challenges, 14 $4^{\text {th }}$ Annual World Preclinical Congress Conference, (Jun 2015) Focus on Novel Preclinical Models in Oncology and Targeting Histone Acetylation, Boston, MA

54 ${ }^{\text {th }}$ Annual Meeting of Biophysical Society, (Feb 2010) San Francisco, CA,

\section{HONORS \& AWARDS}

Featured Researcher, WVU Microscope Imaging Facility Newsletter

- My research images were featured in the November issue

Class Honors (Top 15\%), West Virginia University School of Medicine

2010-2011, 2011-2012

- Awarded in Human Function, Public Health, Physical Diagnosis \& Clinical Integration 1, Human Structure, Neurobiology, Pathology, Microbiology, Pharmacology, and Physical Diagnosis \& Clinical Integration 2B

Early Decision Scholar, West Virginia University School of Medicine

- Early acceptance with priority scheduling 
West Virginia University Medical Scholarship, West Virginia University School of Medicine

- Awarded based on merit and financial need; $1 / 2$ tuition waiver

Most Outstanding Senior, West Virginia University

- One of thirty-five WVU graduating seniors of West Virginia University by the WVU Foundation

Phi Beta Kappa Society, West Virginia University Chapter

- Recognizes excellence in liberal arts and sciences

Most Outstanding Chemistry Student, WVU College of Arts and Sciences

- Only one student per major chosen

Phi Lambda Epsilon Member, West Virginia University

- WVU Chemistry Honorary

Eberly College of Arts and Sciences Certification of Achievement Recipient, WVU

- Undergraduate and Graduate Division

West Virginia PROMISE Scholarship Recipient, West Virginia University

WVU Presidential Award for Excellence in Scholarship, West Virginia University

$2006-2010$

- 4.0 GPA for four semesters

Outstanding Junior Chemistry Major, ACS Northern WV Section

$1^{\text {st }}$ Place in WVNano Initiative Research Symposium, West Virginia University

- Undergraduate Division

$1^{\text {st }}$ Place in WVU ACS Student Affiliates Chemical Symposium, WVU

- Undergraduate and Graduate Division

\section{LEADERSHIP EXPERIENCE}

President of WVU American Physician Scientist Association Chapter

2011-2015, 2015-2016

- Member from 2011-present, President from 2015-20016

Pre-Health Advisor Workshop, WVU School of Medicine

- Panelist

President/Member of WVU Neurology Specialty Group

2011-2012, 2010-2011

- Member from 2010-2011, President from 2011-2012

President/ Member of WVU American Chemical Society Student Affiliates

$2007-2010$

- Secretary for 2008-2009, President from 2009-2010

Coordinator of Chemistry Learning Center

$2007-2010$

- Volunteer teaching Chemistry 115 and 116 and coordinating tutors

Officer in WVU Undergraduate Mortar Board Honorary

- Active leader in service events, social events and academic endeavors 
MD/PhD Student Admissions Committee

2012-2013, 2015-2016

- Interviewed perspective incoming $\mathrm{MD} / \mathrm{PhD}$ candidates

Cancer Cell Biology Faculty Meeting Student Representative

- Advocate for student issues

Eberly College Outstanding Teacher Award Committee Member

2009-2010

- Advocate for student issues

\section{TEACHING EXPERIENCE}

Mentorship of Undergraduate Researchers, West Virginia University School of Medicine

2012-2016

- Mentor of multiple undergraduate Biology Honor Thesis students

Peer Advisor for MS1s, WVU School of Medicine

- Mentor to MS1 WVU medical students

Head Tutor for Academic Resource Center, West Virginia University

- Chemistry tutor/teacher for WVU students

Tutor for Chemistry Learning Center, West Virginia University Chemistry Department

- Chemistry tutor/teacher for WVU students

Teaching Assistant, West Virginia University Chemistry Department

- Teaching Assistant for Honors Chemistry class

\section{WORK EXPERIENCE}

Pifers' Service Center, Mineral Wells, WV

$2003-2010$

- Light mechanic, cashier, CDL wrecker driver

Tutor at Academic Resource Center, Morgantown, WV

$2008-2010$

- Tutor from 2008-2009, Head tutor from 2009-2010

Undergraduate Researcher, WVU Honors Sure, Morgantown, WV

- Researching using Atomic Force Microscopy

Teaching Assistant in WVU Chemistry Department, Morgantown, WV

- Teaching assistant for honors CHEM 117 


\section{Community Service}

- Bartlett House: Volunteer on Wednesday evenings in the kitchen to prepare dinner for residents of this transitional housing in Morgantown, WV

- The MD-PhD Track Presentations for multiple undergraduate programs

- WVU Fly-fishing Club, Get Trashed Event- picking up trash on Decker's Creek

- SAFE Clinic: participant; student and faculty run free health clinic

- Class Trip to New Orleans, LA: Service work with Habitat for Humanity in the Lower Nine

- Coordinator of the Chemistry Learning Center

*Over 200 hours logged in undergraduate; 140+ hours in medical school

\section{PERSONAL INTERESTS \& HOBBIES}

Fly-fishing, traveling, backpacking, reading, and scuba diving 


\section{Appendix AI}

Cieply B., Riley P., Pifer PM., et al., Suppression of the Epithelial-Mesenchymal Transition by Grainyhead-like-2. Cancer Research 72(9); 2440-53. 


\title{
Suppression of the Epithelial-Mesenchymal Transition by Grainyhead-like-2
}

\author{
Benjamin Cieply ${ }^{1}$, Philip Riley IV ${ }^{1}$, Phillip M. Pifer ${ }^{1}$, Joseph Widmeyer ${ }^{1}$, Joseph B. Addison ${ }^{1}$, Alexey V. Ivanov ${ }^{1,2}$, \\ James Denvir ${ }^{3}$, and Steven M. Frisch ${ }^{1,2}$
}

\begin{abstract}
Grainyhead genes are involved in wound healing and developmental neural tube closure. In light of the high degree of similarity between the epithelial-mesenchymal transitions (EMT) occurring in wound-healing processes and the cancer stem cell-like compartment of tumors, including TGF- $\beta$ dependence, we investigated the role of the Grainyhead gene, Grainyhead-like-2 (GRHL2) in oncogenic EMT. GRHL2 was downregulated specifically in the claudin-low subclass breast tumors and in basal-B subclass breast cancer cell lines. GRHL2 suppressed TGF- $\beta$-induced, Twist-induced or spontaneous EMT, enhanced anoikis sensitivity, and suppressed mammosphere generation in mammary epithelial cells. These effects were mediated in part by suppression of ZEB1 expression via direct repression of the ZEB1 promoter. GRHL2 also inhibited Smad-mediated transcription and it upregulated mir-200b/c as well as the TGF- $\beta$ receptor antagonist, BMP2. Finally, ectopic expression of GRHL2 in MDA-MB-231 breast cancer cells triggered an MET and restored sensitivity to anoikis. Taken together, our findings define a major role for GRHL2 in the suppression of oncogenic EMT in breast cancer cells. Cancer Res; 72(9); 2440-53. (C)2012 AACR.
\end{abstract}

\section{Introduction}

The oncogenic epithelial-mesenchymal transition (EMT) is a transcriptional reprogramming event that endows restricted subclasses of tumor cells with enhanced metastatic and stem cell-like properties. These properties include increased migration/invasion, chemo- and radiation resistance, anoikis resistance, and extraordinary tumor initiation frequency, a cancer stem cell-like capability $(1,2)$. In breast cancer, 2 subclasses of tumors, "claudin-low" and "metaplastic", exhibit frank EMTlike gene expression signatures. These are among the most aggressive and least treatment-responsive tumors (3).

In general, TGF- $\beta$ signaling can either promote or suppress tumorigenicity and progression $(4,5)$. It can suppress tumors through Smad-mediated induction of cyclin-dependent kinase inhibitor genes such as p15, and inactivating mutations in this pathway occur in certain tumors. In other contexts, however, TGF- $\beta$ can promote tumor progression by supporting oncogenic EMT induction, through both transcriptional and nontranscriptional mechanisms. For example, in claudin-low and metaplastic mammary tumors-as well as in the stem cell-like $\mathrm{CD} 44^{\text {high }} / \mathrm{CD} 24^{\text {low }}$ tumor cell subpopulation fractionated from breast tumors of other subclasses-TGF- $\beta$ pathway compo-

Authors' Affiliations: ${ }^{1}$ Mary Babb Randolph Cancer Center, ${ }^{2}$ Department of Biochemistry, West Virginia University, Morgantown; and ${ }^{3}$ Department of Biochemistry and Microbiology, Marshall University, Huntington, West Virginia

Corresponding Author: Steven M. Frisch, West Virginia University, PO Box 9300, Morgantown WV 26506. Phone: 304-293-2980; Fax: 304-2934667; E-mail: sfrisch@hsc.wvu.edu

doi: 10.1158/0008-5472.CAN-11-4038

(C)2012 American Association for Cancer Research. nents are strikingly upregulated and, indeed, TGF- $\beta$ receptor kinase inhibitors partially revert the EMT-related gene expression profile $(3,6)$. This indicates a protumorigenic role of the TGF- $\beta$ pathway in this context.

Cell culture models confirm these conclusions. An extensively characterized SV40 large T/hTERT-immortalized mammary epithelial cell line, HMLE, exhibits spontaneous EMT in a subpopulation of $\mathrm{CD} 44^{\text {high }} / \mathrm{CD} 24^{\text {low }}$ cells $(7)$. This EMT is accompanied by upregulation of autocrine TGF- $\beta$ signaling, mainly through downregulation of antagonists such as BMP2/ 4. Reversion to an epithelial phenotype could be achieved by inhibition of this signaling pathway. Moreover, even Twistinduced EMT in this system was partially dependent upon autocrine TGF- $\beta$ signaling, showing its functional significance in multiple contexts.

The similarities between oncogenic and wound-healingrelated EMT, including TGF- $\beta$ dependence, have been documented (8-10). In this light, we hypothesized that perhaps other "dedicated" wound-healing genes might prove important in oncogenic EMT. Grainyhead family genes have been shown to play an important role in wound healing, epidermal integrity, and the mechanistically related process of embryonic neural tube closure (11-14). Relevant, albeit limited, Grainyhead family target genes identified so far include E-cadherin, claudin-4, desmoglein-1, transglutaminase-1, rho-GEF19, several Zelda-targeted genes expressed during the maternalzygotic transition (MZT) in Drosophila, and telomerase (11, 12, 14-17). With regard to cancer, Grainyhead-like-2 (GRHL2) gene amplification has been noted in several tumor types, including breast cancer, and suppressed death receptor expression, conferring resistance to apoptosis mediated by the corresponding ligands, indicating that GRHL2 was a 
potential oncogene (18). On the other hand, GRHL3 was recently shown to suppress squamous cell carcinoma, due to its activation of PTEN expression (19).

Inspired by its role in wound healing, we hypothesized and report here that GRHL2 suppressed EMT mediated by the TGF$\beta$ signaling pathway. Consistent with this effect, GRHL2 was downregulated specifically in EMT-dependent mammary tumors (claudin low, metaplastic) and cell lines (basal-B). ZEB1 was found to be required for EMT and was a direct target for repression by GRHL2. GRHL2 also enhanced anoikis sensitivity. These data suggest an EMT-suppressive function of GRHL2 that is downregulated in the context of TGF- $\beta /$ EMTdriven tumor types.

\section{Materials and Methods}

\section{Cell lines}

HMLE, HMLE + Twist-ER, and HMLE + Ras cells (HMLER) were generous contributions from R. Weinberg (The Whitehead Institute, Cambridge, MA). HMLE and HMLE + Twist-ER were grown in a 1:1 mixture of Mammary Epithelial Growth Medium (MEGM; Lonza) and [Dulbecco's Modified Eagle's Medium (DMEM): Ham's F-12 (Gibco) + 5\% horse serum + $1 \times$ penicillin-streptomycin-glutamine (PSG) $+10 \mu \mathrm{g} / \mathrm{mL}$ insulin, $10 \mathrm{ng} / \mathrm{mL}$ EGF, $0.5 \mu \mathrm{g} / \mathrm{mL}$ hydrocortisone], where indicated, 4-hydroxytamixofen $(10 \mathrm{ng} / \mathrm{mL})$ was added to the HMLE + Twist-ER cells to activate the Twist-ER protein. HMLER cells were grown in MEGM. MDA-MB-231LN were provided by E. Pugacheva (West Virginia University) and were grown in advanced DMEM (Invitrogen) $+10 \%$ FBS $+1 \times$ penicillinstreptomycin-glutamine (PSG).

\section{Generation of stable cell lines by retroviral transduction} Human GRHL2 was amplified from a template purchased from Open Biosystems (MHS4426-99625903) and subcloned by standard molecular biology methods into the pMIG or MSCVIRES-puro retrovirus (Addgene; XhoI site). Retroviruses were packaged and amplified in GP2 + 293T cells by transfection of $4.5 \mu \mathrm{g}$ of retroviral plasmid and $2.5 \mu \mathrm{g}$ of pCMV-VSV-G per 60 $\mathrm{mm}^{2}$ dish of cells, with Mirus TransIT reagent. Viral supernatants were collected 48 hours later, filtered through 0.45micron filters (Whatman) and $0.6 \mathrm{~mL}$ of supernatant was used to infect 1 well of a 6 -well dish of target cells by centrifugation at 1,400 rpm for 1 hour followed by 6 hours to overnight incubation. Infected cells were either selected for puromycin ( $2 \mu \mathrm{g} / \mathrm{mL}$ for HMLE, $0.5 \mu \mathrm{g} / \mathrm{mL}$ for MDA-MB-231) or flow sorted for GFP, followed by Western blot analysis to verify expression.

\section{Generation of stable cell knockdown cell lines by lentiviral transduction and transient knockdown (siRNA)}

GRHL2, ZEB1, and scrambled control short hairpin RNA (shRNA) were purchased from Open Biosystems in the pGIPZ vector. (catalog numbers: shGRHL2a: RHS4430-99291384; shGRHL2b: RHS4430-99614394 and shZEB1a: V3LHS_356186; shZEB1b: 3 V3LHS_356187; the latter were also subcloned into vector pTRIPz using Mlu1/Xho fragments). SiZEB1 Smartpool was from Dharmacon catalog number (L-006564-01-0005) and was transfected transiently using Lipofectamine RNAi-max (Invitrogen).

\section{Mammosphere assay}

Mammospheres were seeded at $1 \times 10^{4}$ cells per well of a 6 well Ultra Low Cluster Plate (Corning) and grown for 7 to 10 days in the appropriate growth media $+0.5 \%$ methylcellulose (MC). Wells were fed every third day with $1 \mathrm{~mL}$ media + MC. Total mammospheres per well were counted and the size cutoff value was set at $150 \mathrm{~mm}$ in diameter; the same cells were plated at $2 \times 10^{5}$ cells per well of a 6 -well plate and the number of cells was counted each day for 4 days, to measure normal growth rate. Error bars represent the SD of replicates.

\section{Anoikis assays}

Cells were dissociated using TrypLE Express (Invitrogen), counted and a fixed amount $1 \times 10^{5}$ to $2 \times 10^{5}$ cells per well of a 6-well Ultra-Low Attachment Cluster Dish (Costar) were suspended in normal growth media $+0.5 \% \mathrm{MC}$ for the indicated for 6 to 24 hours to induce anoikis. For cell death ELISA (CDE) analysis of apoptotic DNA fragmentation, the cells were collected in 3 volumes of media and then spun down at 1,500 rpm for 3 minutes. The pellet was then washed with D-PBS (Invitrogen) transferred to a microfuge tube, pelleted at 7,000 rpm for 15 seconds, and lysed in PBS $+0.5 \%$ Triton X-100 +10 mmol/L EDTA (note: the lysis buffer with the Cell Death ELISA kit from Roche was found to lyse cells inadequately). Lysates were incubated on ice for 15 minutes with occasional mixing and then centrifuged at $13,000 \mathrm{rpm}$ for 12 minutes. The supernatants were subjected to the CDE according to the manual provided with the kit (Roche). Alternatively, percentage of cell death was determined by a Trypan blue exclusion assay, wherein cells were suspended in same manner but collected and resuspended in Accumax (Innovative Cell Technology) to ensure a single-cell suspension. After brief incubation (2-3 minutes), Trypan blue was added to this solution and the percentage of dead cells were immediately counted on a hemocytometer. All samples were analyzed in either duplicate or triplicate, and time-zero cell death values were subtracted from the data presented here.

\section{Three-dimensional culture}

Three-dimensional (3D) Matrigel culture methods were adapted from (20). To summarize, $2.5 \times 10^{3}$ cells per well were seeded onto 8-well chamber slides (Labtek) where $45 \mu \mathrm{L}$ Matrigel (Trevigen) had been evenly distributed. The cells were overlaid with the appropriate growth media (see above) $+2.5 \%$ Matrigel. After 6 days in culture 3D migration/invasion was quantified by counting the number of structures that had formed protrusions versus those that grew as lobular structures defined by their contact with matrix (see images for clarification). At least 200 structures were counted per experiment; error bars represent the SD across 3 samples.

\section{Microarray methods}

RNA isolated by RNeasy Plus kit (Qiagen) was quantified using Nanodrop (Fisher Scientific). The RNA quality was check 
on Bioanalyzer (Agilent). Two hundred fifty nanograms of each RNA sample with an RNA integrity number (RIN) value greater than 7 was processed by the Ambion WT Expression Kit according to the manufacturer's instructions. The typical yield from the reaction was 6 to 9 micrograms of cDNA. The required amount of cDNA $(5.5 \mu \mathrm{g})$ was processed for fragmentation and biotin labeling by the GeneChip WT Terminal Labeling Kit (Affymetrix). The efficiency of fragmentation reaction was checked via Agilent Bioanalyzer. The entire reaction of fragmented and biotin-labeled cDNA $(50 \mu \mathrm{L})$ with added hybridization controls was hybridized to the human GeneChip 1.0 ST Exon Arrays (Affymetrix) at $45^{\circ} \mathrm{C}$ for 17 hours in GeneChip Hybridization Oven 640 (Affymetrix). Human GeneChip 1.0 ST Exon Arrays were stained with FS 450_0001 protocol in Affymetrix GeneChip Fluidics Station 450. Briefly, biotin-labeled cDNA was reacted using 2 rounds of washes with a solution containing a streptavidin-phycoerythrin complex, with an intermediate treatment of biotin-labeled antistreptadvidin antibody to amplify the signal. Phycoerythrin labeling was detected within the Affymetrix GeneChip Scanner 3000 7G plus using $532 \mathrm{~nm}$ light and detected by a photomultiplier tube. Expression Console software (Affymetrix) was used to check quality controls of hybridized chips. All chips that passed quality controls were RMA normalized with Expression Console software. The microarray data were deposited into the NCBI GEO database as accession number GSE36081.

To examine the extent to which GRHL2 affects the propensity to undergo epithelial-mesenchymal transition (EMT), we compared the relative expression of genes in an identified EMT signature (21) to the relative expression of genes in cells with constitutive GRHL2 expression. Specifically, we obtained the expression profile of the 251 Core EMT signature genes from Supplementary Table S1 (21) and computed the mean log ratio of the relative expression. We restricted the genes to those that appeared on our array platform and computed the Pearson correlation coefficient of those genes to the log expression ratio of GRHL2-regulated genes compared with the control.

\section{Reporter assays}

HMLE were transiently transfected using Lipofectamine 2000 (Invitrogen) at a $1 \mu \mathrm{g}$ DNA: $2 \mu \mathrm{L}$ Lipofectamine ratio. A total of $1.5 \mu \mathrm{g}$ DNA per well of a 12-well plate was the maximum amount of DNA found to be tolerable. Transfection mixtures were incubated for 20 minutes in $200 \mu \mathrm{L}$ Opti-MEM (Invitrogen) and then added to the cells in normal growth media lacking antibiotics. Cells were incubated for 4 hours then refed with normal growth media. Lysates were made by washing the cells once with PBS then lysing in $1 \times$ Cell Culture Lysis Buffer (Promega). Lysates were centrifuged at $13,000 \mathrm{rpm}$ for 10 minutes, and the supernatants were assayed for luciferase and $\beta$-galactosidase activity as internal control. Luciferase assay reagent was obtained from Promega and the $\beta$-galactosidase $2 \times$ assay reagent was $200 \mathrm{mmol} / \mathrm{L}$ sodium phosphate $(\mathrm{pH} 7.3)$, $2 \mathrm{mmol} / \mathrm{L} \mathrm{MgCl}_{2}, 100 \mathrm{mmol} / \mathrm{L} \mathrm{2-mercaptoethanol,} \mathrm{and} 1.33$ $\mathrm{mg} / \mathrm{mL} o$-nitrophenylgalactoside. The Smad reporter construct 3TP-Lux was from Addgene. The ZEB1 promoter-luciferase construct in pGL3 (22) was kindly provided by Antonio
Garcia de Herreros (Universitat Pompeu Fabra, Barcelona, Spain). CMV-LacZ or TK-LacZ were used as internal controls. The GRHL2 clone was purchased from Open biosystems, catalog no MHS4426-99625903, and the coding sequence was cloned into the XhoI site of pcDNA3.1+. Subfragments of the ZEB1 promoter were generated and cloned into pGL3-promoter (MluI-BglII) using the following primers: fragment 1 : ZPfrl-f: Ttaat ACGCGT CCTTAAGGTCCTGCACGGCG, ZPfr1r: tatat agatct AAGTTCCGCTTGCCAGCAGC; fragment 2: ZPfr2-f: TtaatACGCGT CTAGCCTCTCTTTCAATCCA, ZPfr2r: tatatagatctTCCGCCCCCCGCACCCCGGGGC; fragment 3: ZPfr3-f: TtaatACGCGTCACGCGAGGCGTGGGACTGA, ZPfr3r: tatat agatct GGATGCCGGGAAACCGTAGG; fragment 4: ZPfr4-f: Ttaat ACGCGT GCCTCCCTCTCCCCACCACA, ZPfr4-r: tatat agatct ACCGCACCTGGTTTACGACA; fragment 5: ZPfr5-f: TtaatACGCGT GCGGACCGGGTGTGGGAGGC, ZPfr5-r: tatat agatct TACCTGACCCGCGCAGCCCG; fragment 6: ZPfr6-f: Ttaat ACGCGT GCCTCTCTCCGGTCGCCGCG, ZPfr6-r: tatatagatct GGGGCAGGGAGGGATCTGGC, fragment 7: ZPfr7-f: Ttaat ACGCGT GGGCAGCCGCGGCGGGTGTG, ZPfr7-r: tatat agatct ACCGTGGGCACTGCTGAATT.

The primers used to mutate the fragment 4 construct (using Stratagene Quickchange II XL kit) were: Mut-f, gtaaagccgggagtgtcgtcccacaggtgcggtagatctgcg; and Mut-r, cgcagatctaccgcacctgtgggacgacactcceggctttac.

\section{Immunofluorescence}

For Smad2 localization, TGF- $\beta$ ( $5 \mathrm{ng} / \mathrm{mL})$ was added for 6 hours and the coverslips were fixed with $4 \%$ paraformaldehyde (PFA) in PBS for 10 minutes. PFA was quenched with 100 $\mathrm{mmol} / \mathrm{L}$ glycine in PBS. Cells were permeabilized with $0.2 \%$ Triton X-100 in PBS at 4 degrees for 10 minutes, washed twice with PBS, and blocked for 1 hour in: PBS $+10 \%$ goat serum + $0.1 \%$ Tween $-20+0.1 \%$ BSA. Primary and secondary antibodies were diluted in blocking buffer. Primary antibodies were as follows: SMAD2; Cell Signaling, rb, 1:200. Secondary: rb Alexa 555 (red); Molecular Probes, 1:1,000. Mounting media: Prolong Gold w/DAPI (Invitrogen).

For E-cadherin, vimentin, and GRHL2 the cells were fixed in $100 \%$ methanol at $-20^{\circ} \mathrm{C}$ for at least 1 hour. They were then washed twice with PBS and blocked as earlier. E-cadherin, ms (BD Biosciences), 1:200; Vimentin, rb (Cell Signalling), 1:200; GRHL2, rb (Sigma), 1:200. Secondaries used were anti-mouse Alexa 555 (red) or anti-rabbit Alexa 488 (green) or A555 (red); Molecular Probes, diluted 1:1,000. Coverslips were mounted in Prolong Gold as above. Images were produced with the Axiovert 200M microscope, AxioCam MRM camera, and Axio Vision 4.3.1 software (Zeiss).

\section{Chromatin immunoprecipitation}

A total of five $100-\mathrm{mm}^{2}$ dishes of 4-hydroxytamoxifen (4-OHT)-induced HMLE + Twist-ER (+GRHL2, in some experiments) were each fixed in $1.2 \mathrm{~mL} 10 \%$ electron microscopy grade PFA for 10 minutes. Following quenching with glycine, chromatin immunoprecipitation (ChIP) was conducted exactly as described previously (23) with the following antibodies, $3.3 \mu \mathrm{g}$ each: GRHL2 (Sigma); Histone H3 (Cell Signaling), or nonimmune rabbit IgG (Pierce). 


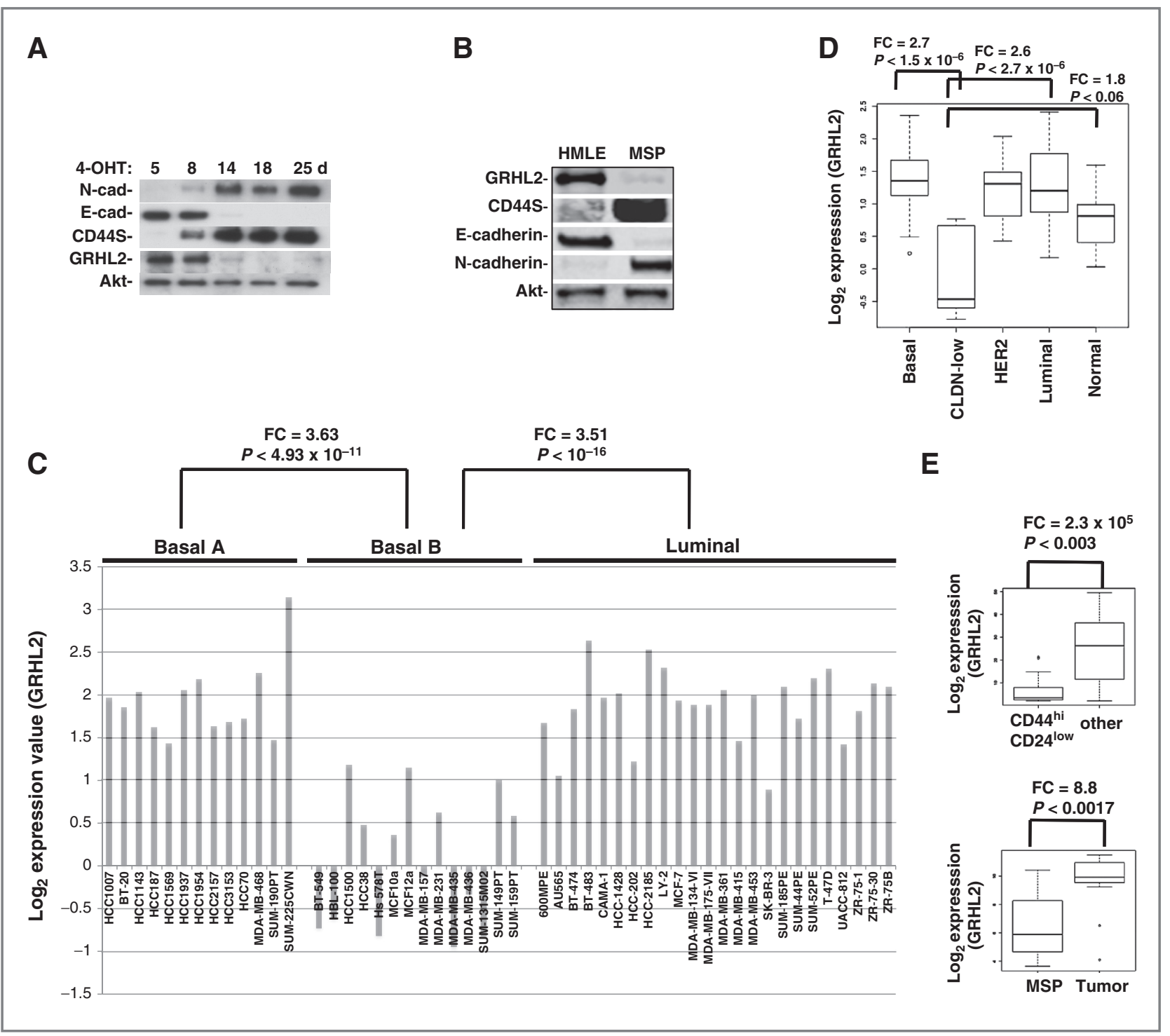

Figure 1. GRHL2 is downregulated in cells and tumors that have undergone EMT. A, Twist downregulates GRHL2. HMLE cells with tamoxifen-inducible Twist (Twist-ER) were induced with 4-hydroxytamoxifen (4-OHT) for the indicated periods of time and analyzed for epithelial, mesenchymal, and tumor-initiating cell (CD44) marker genes. B, GRHL2 is downregulated in the MSP cells relative to parental HMLE cells. C, GRHL2 is downregulated specifically in "basal-B" subclass of breast cancer cell lines characterized by Neve and colleagues (42). D, GRHL2 is downregulated specifically in the "claudin-low" subclass of breast cancer characterized by Hennessy and colleagues (3). E, GRHL2 is downregulated specifically in the tumor-initiating/MSP characterized by Creighton and colleagues (25).

ChIP-derived DNA was analyzed by PCR using the following primer sets: ZEBf6, GCGAGGCGTGGGACTGATGG; ZEBr6, AAAGTTGGAGGCTCGGCGGC; ZEBf10, CTGCACGGCGATGACCGCT; ZEBr10, TTCCGCTTGCCAGCAGCCTC; GAPDH-f, ATGGTTGCCACTGGGGATCT; and GAPDH-r, TGCCAAAGCCTAGGGGAAGA. For quantitative PCR analysis, ChIP DNAs were analyzed using $2 \times$ Power-Sybr Green Master Mix (Applied Biosystems) and signals were calculated relative to input DNA using the " $\Delta \Delta C_{\mathrm{t}}$ " method; a correction of $1.5 \times$ was applied to adjust for the higher efficiency of glyceraldehyde-3-phosphate dehydrogenase (GAPDH) amplification compared with ZEB1 amplification obtained in a control reaction using lysates from GRHL2-null cells.
The primer set used was F8, GCCGCCGAGCCTCCAACTTT; and R8, TGCTAGGGACCGGGCGGTTT.

\section{Western blotting}

SDS-PAGE was conducted using $4 \%$ to $20 \%$ gradient Tris Glycine gels (Invitrogen). Proteins were immobilized by electophoretically transferring them to a polyvinylidene difluoride filter (Immobilon) in 5\% MeOH containing Tris-Glycine transfer buffer. Filters were blocked in PBS $+5 \%$ nonfat milk, primary antibodies were incubated in PBS $+0.1 \%$ Tween-20 $+5 \%$ nonfat milk, secondary antibodies were incubated in PBS $+0.1 \%$ Tween- $20+5 \%$ milk $+0.01 \%$ SDS. Primary antibodies were typically incubated for 2 hours to overnight, secondary 


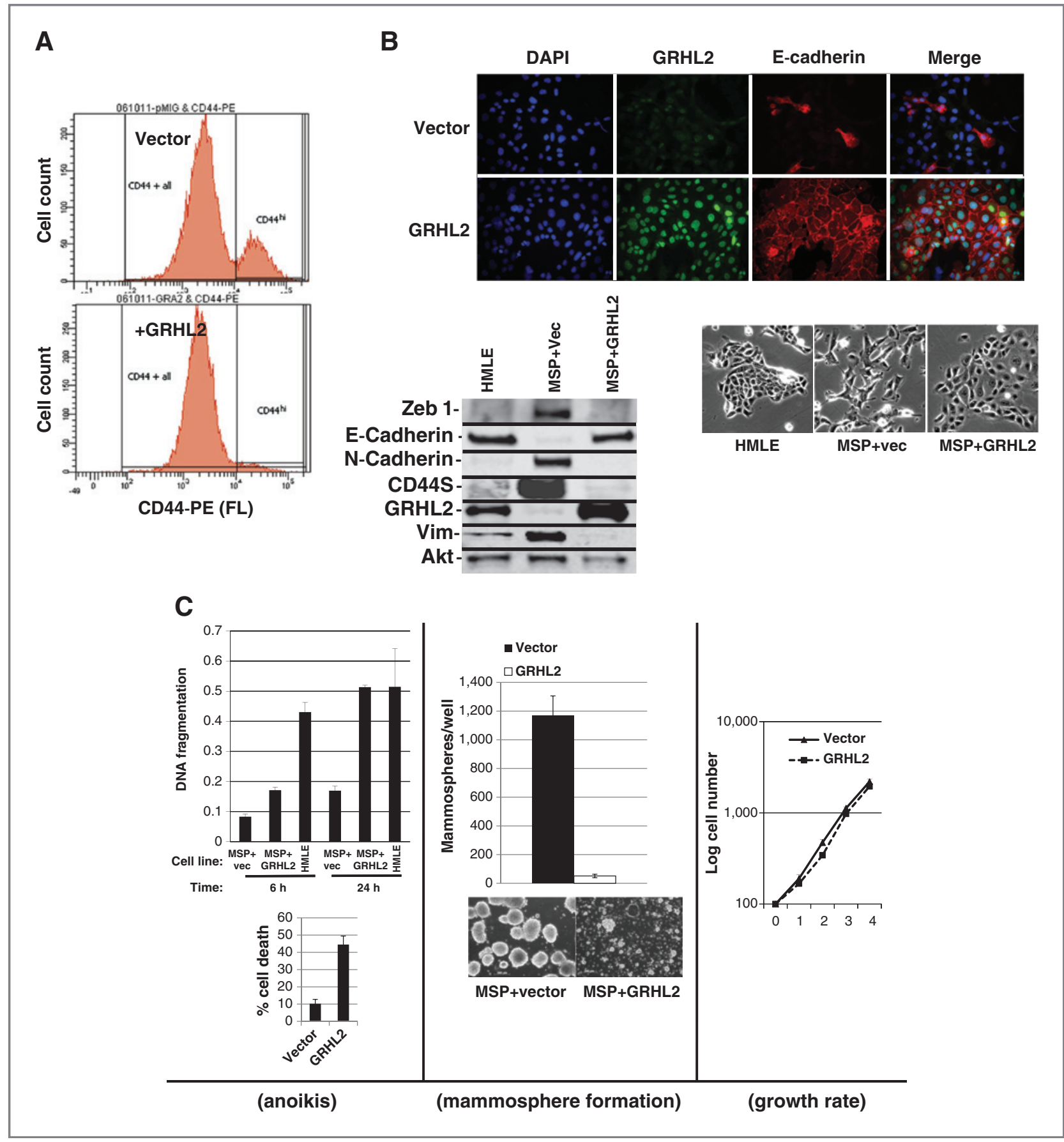

Figure 2. GRHL2 suppresses EMT. A, ectopic expression of GRHL2 suppresses the CD44 expression in the spontaneously occurring MSP of HMLE cells. HMLE + Twist-ER cells (without 4-OHT) infected with GRHL2 or empty retroviral vector (pMIG) were analyzed for CD44 expression by FACS. B, GRHL2 reverts MSP cells to an epithelial phenotype. The CD44 ${ }^{\text {high }}$ MSP of HMLE, obtained by flow sorting of HMLE cells, were infected with empty vector (vec) or GRHL2 and then (top) stained for the indicated proteins by immunofluorescence or (bottom) probed for epithelial and mesenchymal marker genes by Western blotting. C, GRHL2 expression in MSP cells restores anoikis sensitivity (left, top graph represents a DNA fragmentation ELISA assay; bottom graph represents a Trypan blue permeability assay) and reduces mammosphere (middle), without affecting growth rate (right).

antibodies were incubated for 1 hour. Primaries used were: E-cadherin, ms (BD Biosciences); Vimentin, ms [Santa Cruz Bio-Tech (SCBT)]; N-cadherin, ms (BD Biosciences); CD44 (HCAM), ms (SCBT); ESRP1/2, ms (Contributed by Russ Carstens, University of Pennsylvania, Philadelphia, PA); Actin, ms
(Millipore); Akt, rb or ms (Cell Signaling); GRHL2, rb (Sigma); Zebl, rb (Sigma) or rb (Cell Signaling); Ankyrin-G, rb (S.M.F. custom generated; ref. 23); total-Smad2/3,ms (BD Biosciences); phospho-Smad2/3, rb (Cell Signaling); NF2 (merlin), rb (SCBT). Secondary antibodies for chemiluminescence were either anti- 


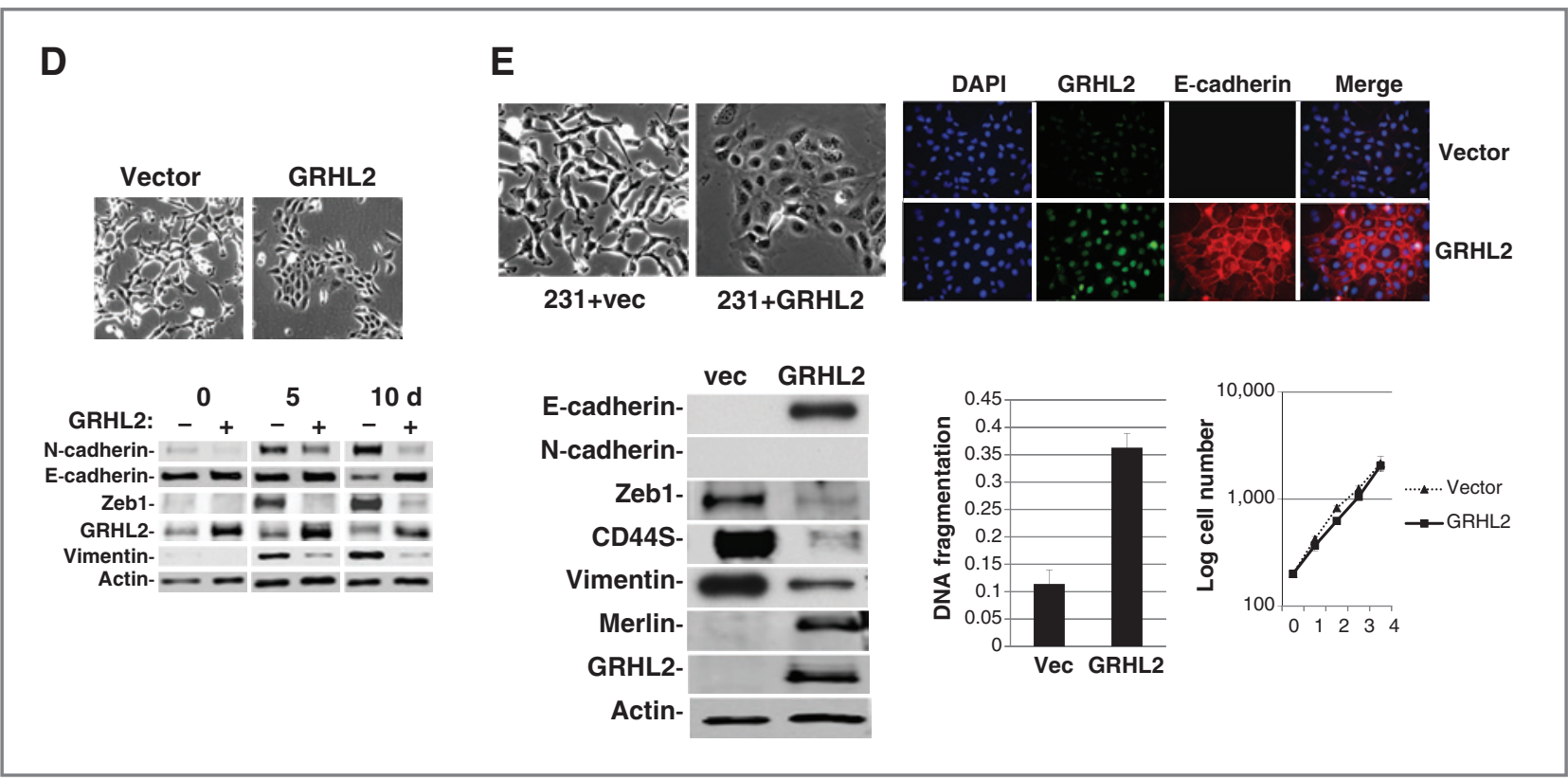

Figure 2. (Continued) D, GRHL2 suppresses Twist-induced EMT. HMLE + Twist-ER expressing either empty vector or GRHL2 were treated with 4-OHT (to activate the Twist transgene) for 7 days and photographed to record morphology (top). Bottom, time course of changes in epithelial and mesenchymal marker genes after induction of the Twist gene with 4-OHT, in cells with or without ectopic GRHL2 expression. E, GRHL2 suppresses EMT and anoikis resistance in MDA-MB-231LN cells. MDA-MB-231LN expressing either empty vector or GRHL2 were analyzed for morphology (phase contrast microscopy, top left), E-cadherin expression and localization (immunofluorescence, top right), expression of epithelial and mesenchymal markers (Western blotting, bottom left), anoikis sensitivity (DNA fragmentation ELISA, middle), and adherent growth rate (bottom right). DAPI, 4',6-diamidino-2-phenylindole.

mouse or anti-rabbit, conjugated to horseradish peroxidase (Bio-Rad) and were used at 1:3,000 dilution; Western blot analysis were developed using (ECL-West Pico; Pierce). Fluorescently tagged secondary antibodies were mouse IRDye $800 \mathrm{CW}$ or rabbit IRDye 680LT (Li-Cor) used at 10:000 and detected using the Odyssey Infrared Imaging System (Li-Cor). Fluorescent images were converted to gray scale.

\section{Other bioinformatics methods}

We obtained GRHL2 expression data from NCBI Gene Expression Omnibus (accession number GSE10885; ref. 3). We compared the log expression of GRHL2 among the tumor types basal, claudin, Her2, luminal, and normal. We carried out a Tukey HSD test to determine which comparisons among these groups showed statistically significant differences; family-wise 95\% confidence intervals for the log expression were plotted. Statistical analyses were conducted with $\mathrm{R}$ version 2.13.1 (www.r-project.org).

\section{Results}

\section{Loss of GRHL2 expression is associated with a mesenchymal phenotype}

On the basis of the specific expression of GRHL2 in mouse epithelia $(12-14,24)$, we investigated its potential regulation during EMT. Using HMLE cells that express a Twist-ER fusion-a previously characterized model of inducible EMT (21) - we analyzed the levels of GRHL2 over a time course of Twist induction by tamoxifen and found that, indeed, GRHL2 protein was downregulated during EMT with kinetics similar to the loss of E-cadherin and gain of $\mathrm{N}$-cadherin (Fig. 1A).
Consistent with this finding, spontaneous, stable EMT in a subpopulation of HMLE cells ("mesenchymal subpopulation") obtained by sorting for CD $44^{\text {high }}$ phenotype, described previously (7), also displayed low GRHL2 expression (Fig. 1B).

Breast cancer cell lines have been classified by gene expression profiles. One particular subclass, basal-B, was characterized by a mesenchymal profile. GRHL2 was dramatically downregulated, specifically in this subclass (Fig. 1C). Analogously, the "claudin-low" subclass of mammary tumor samples-also characterized by a mesenchymal gene expression profile and poor prognosis-expressed substantially lower levels of GRHL2 than other subclasses, which, in fact, showed a modest upregulation, compared with normal breast tissue (Fig. 1D, and see Discussion). GRHL2 was also downregulated dramatically in a different tumor type characterized by EMT, clear cell renal carcinoma (Supplementary Fig. S1). Moreover, chemoresistant subpopulations of primary breast tumor cells obtained after chemotherapy of patients by sorting for $\mathrm{CD} 44^{\text {high }} / \mathrm{CD} 24^{\text {low }}$ marker expression or mammosphere generation (25) expressed decreased levels of GRHL2 as well (Fig. 1E).

These data suggested that GRHL2 loss is a widespread characteristic of both primary and cultured tumor cells that have undergone EMT and acquired a tumor-initiating phenotype, informed the hypothesis that GRHL2 downregulation was functionally important for EMT.

\section{GRHL2 is an EMT suppressor}

The spontaneously occurring, $\mathrm{CD} 44^{\text {high }} \mathrm{CD} 24^{\text {low }}$ mesenchymal subpopulation (MSP) cells within the heterogeneous HMLE cell line are characterized by EMT, attributed to 


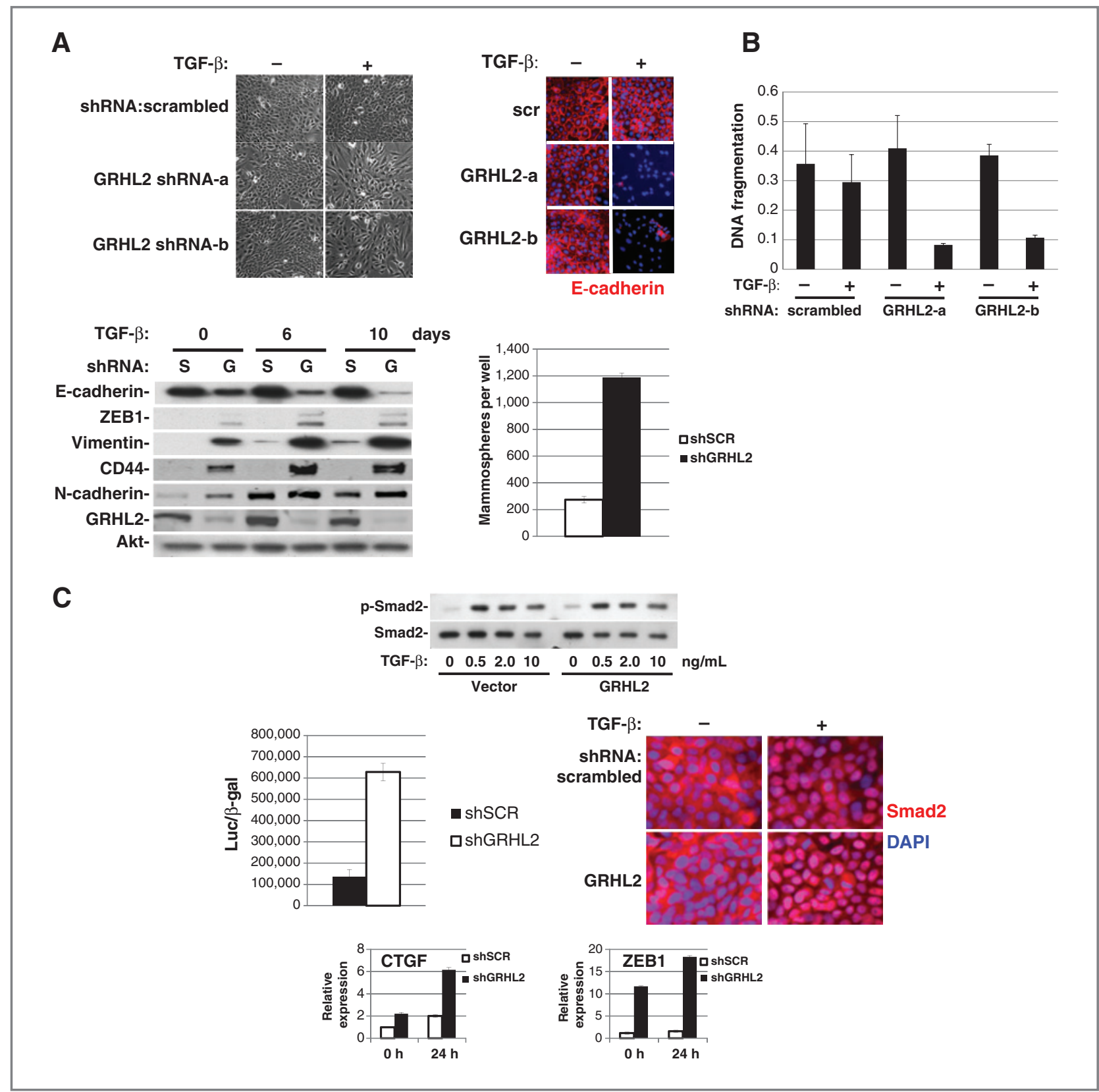

Figure 3. GRHL2 suppresses TGF- $\beta$-induced EMT. A, GRHL2 knockdown permits HMLE cells to undergo TGF- $\beta$-induced EMT. HMLE + Twist-ER cells (no 4-OHT) with 2 distinct GRHL2 shRNAs or a scrambled control shRNA were exposed to TGF- $\beta$ for 2 weeks before analysis for cell morphology (top left), E-cadherin expression/localization (immunofluorescence, top right) or mammosphere generation (graph, lower right). Epithelial and mesenchymal markers were also analyzed at the indicated times of TGF- $\beta$ treatment (Western blot, bottom left). B, GRHL2 suppresses the ability of TGF- $\beta$ to confer anoikis resistance. The cell lines described earlier were assayed for anoikis by DNA fragmentation after 2-week incubation with or without TGF- $\beta$. C, GRHL2 inhibits Smad-mediated transcription. Left, effect of stable GRHL2 knockdown on activity of a Smad-responsive reporter construct (3TP-lux) was determined by luciferase assays of transiently transfected HMLE + Twist-ER cells (no 4-OHT) expressing either scrambled or GRHL2a shRNA that were treated with TGF- $\beta$ for 16 hours before lysis; values are luciferase activity normalized to an internal cotransfected $\beta$-galactosidase control. Bottom, effect of stable GRHL2 knockdown on induction of TGF- $\beta$ /Smad target genes, CTGF and ZEB1, by exogenous TGF- $\beta$, determined by qRT-PCR. Top, no effect of GRHL2 on phosphorylation of Smad2/3. MSP cells expressing empty vector or GRHL2 were treated with the indicated concentrations of TGF- $\beta$ for 24 hours and analyzed for total and p-Smad2/3. Right, no effect of GRHL2 on nuclear translocation of Smad2. The GRHL2 knockdown or control cells described in part (A) were treated with TGF- $\beta$ for 6 hours and analyzed for Smad2 localization by immunofluorescence.

autocrine TGF- $\beta$ signaling pathway activation (7). Infection of HMLE cells with a GRHL2 expression construct and selection for the infected cells using a GFP marker caused the disappearance of the CD $44^{\text {high }}$ subpopulation (Fig. 2A) within a few days after infection, suggesting a conversion effect rather than selective growth (as shown later).

To further characterize this phenomenon, we isolated MSP cells from the HMLE cell line by flow sorting and 


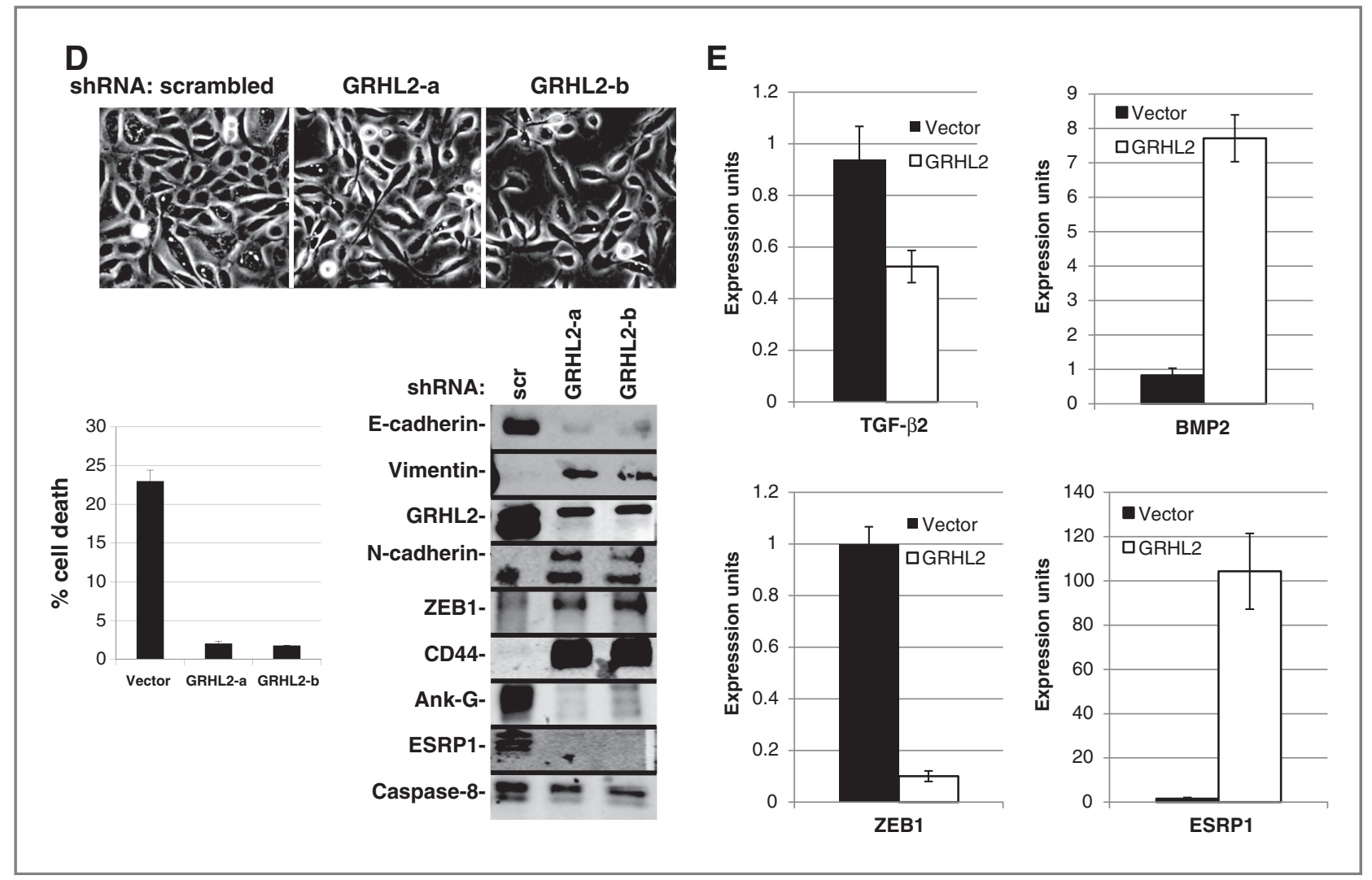

Figure 3. (Continued) D, GRHL2 knockdown induces EMT in HMLE cells with Ras (HMLER). HMLER expressing 2 distinct GRHL2 shRNAs or scrambled shRNA control were imaged with phase contrast microscopy (top), immunoblotted for epithelial and mesenchymal markers (right), or assayed for anoikis (Trypan blue exclusion, left). E, GRHL2 upregulates BMP2. RT-PCR was carried out on RNAs from HMLE + Twist-ER cell lines with or without constitutive GRHL2 expression (induced with 4-OHT), using the indicated primer sets.

infected these with the GRHL2 retrovirus. On the basis of Ecadherin immunofluorescence and Western blotting for epithelial and mesenchymal markers, GRHL2 reverted MSP back to an epithelial phenotype (Fig. 2B). Anoikis resistance and the ability to form mammospheres are key characteristics associated with EMT in HMLE cells. GRHL2 expression in the MSP cells restored anoikis sensitivity and reduced mammosphere formation dramatically, without affecting adherent cell growth (Fig. 2C).

These data indicated that GRHL2 reverted the spontaneous EMT and accompanying tumor-initiating cell characteristics of MSP cells. To test the effect of GRHL2 in other scenarios of EMT, we expressed it constitutively in HMLE + Twist-ER cells and in the prototypical EMT-like/ triple-negative breast cancer cell line, MDA-MB-231, where it caused dramatic reversion of EMT and anoikis resistance in both cases (Fig. 2D and E), indicating a surprisingly broad specificity for this effect.

\section{GRHL2 suppresses TGF- $\beta$-induced EMT}

MSP cells and Twist-expressing HMLE cells rely on autocrine TGF- $\beta$ signaling for their maintenance of mesenchymal and tumor-initiating properties (7), suggesting that GRHL2 could be suppressing EMT through this common pathway. We confirmed that Twist-mediated EMT and acquisition of anoi- kis resistance were TGF- $\beta$-dependent by using LY364947, a specific inhibitor of the TGF- $\beta$ R1 kinase activity (Supplementary Fig. S2). Because this inhibitor mimicked the effects of ectopic GRHL2 in some respects, we tested the effect of GRHL2 on TGF- $\beta$-induced EMT.

TGF- $\beta$ alone was previously reported to be insufficient for EMT induction in HMLE, a process requiring activation of multiple pathways (7). When GRHL2 was partially depleted by 2 distinct shRNAs, TGF- $\beta$ alone induced EMT more efficiently than in cells with control shRNA (Fig. 3A). GRHL2 knockdown facilitated several activities of TGF- $\beta$ : induction of a mesenchymal morphology, downregulation of epithelial-specific genes (E-cadherin, ESRP1 and ankyrin-G-an epithelial cytoskeletal protein that regulates anoikis; ref. 23), and upregulation of vimentin as well as the tumor-initiating cell marker, CD44S; surprisingly, TGF- $\beta$ induced N-cadherin partly independently of GRHL2 expression. Coincident with this facilitation of EMT, GRHL2 knockdown also permitted TGF- $\beta$ to confer a mammosphere-generating, anoikis-resistant state (Fig. 3A and B). GRHL2 also suppressed another feature of EMT, the formation of large protrusive structures during the growth of colonies in 3D Matrigel culture, indicative of invasive potential (Supplementary Fig. S3).

These results suggested that signaling downstream of the TGF- $\beta$ receptor might be suppressed by GRHL2. We focused 
on Smad-mediated transcription, as it plays an important, although not exclusive role in the response to exogenous TGF- $\beta$-mediated EMT (4). Using a well-characterized reporter assay (3TP-lux; ref. 4), GRHL2 knockdown stimulated TGF- $\beta$ / Smad-mediated transcription by approximately $4.6 \times$ relative to control cells (Fig. 3C). Correspondingly, GRHL2 knockdown promoted the induction of the TGF- $\beta /$ Smad target genes, CTGF (26), and ZEB1 (27), by exogenous TGF- $\beta$. Surprisingly, there was no discernable effect of GRHL2 on either the phosphorylation or nuclear translocation of Smad2, suggesting that other mechanisms of inhibition were operative (see Discussion). These results indicated that GRHL2 inhibited Smadmediated transcription in response to exogenous TGF- $\beta$.

In HMLE cells expressing low levels of activated K-ras, (HMLER), GRHL2 knockdown sufficed to induce EMT-that is, even without exogenous TGF- $\beta$ based on the criteria used earlier (Fig. 3D). This EMT was clearly dependent upon autocrine TGF- $\beta$ signaling, in that LY364947 reversed it (Supplementary Fig. S4). The TGF- $\beta$ signaling antagonists BMP2 and -4 were previously shown to be downregulated in MSP cells relative to normal HMLE, which promoted autocrine TGF- $\beta$ signaling (7). Interestingly, BMP2 expression was activated by GRHL2 (Fig. 3E), consistent with the idea that GRHL2 suppressed not only TGF- $\beta$ signaling in response to exogenous ligands but also (by comparison with reported data on the same cell lines; ref. 7) autocrine signaling.

\section{GRHL2 represses ZEB-1 expression}

Suppression of EMT by GRHL2 could occur by a diversity of mechanisms. To elucidate one or more of these in an unbiased manner, we conducted a microarray-based gene expression profiling comparing the HMLE-Twist cells with or without GRHL2 expression. This analysis revealed that genes regulated by GRHL2 (GEO database accession number GSE36081) correlated negatively $(R=-0.7508)$ with genes regulated during EMT (by multiple transcription factors) in the HMLE system, validating the EMT-suppressive effect of GRHL2 (Fig. 4A). The genes regulated by GRHL2 included markers of epithelial versus mesenchymal phenotypes, several of which were ZEB1 target genes; transcription factors implicated in the control of EMT were also noted.

Interestingly, one of the major downregulated GRHL2 target genes was the E-cadherin repressor/EMT inducer ZEB1, as shown by RT-PCR (Fig. 3E) and Western blotting in MSP cells (Fig. 2B), MDA-MB-231 cells (Fig. 2E), HMLE + shGRHL2 cells with TGF- $\beta$ (Fig. 3A), HMLER + shGRHL2 cells (Fig. 3D) and HMLE + Twist-ER cells (Fig. 2D). Functional consequences of ZEB1 downregulation (28) such as the upregulation of mir200b/c (Fig. 4B) and ESRP1 (Fig. 3E) were also evident. The downregulation of ZEB1 by GRHL2 was investigated further as a potential mechanism for suppression of EMT.

To determine whether the ZEB1 gene could be a direct target for GRHL2, we cotransfected the previously characterized ZEB1 promoter (22) together with GRHL2 into the MSP cells (which express low endogenous GRHL2). This revealed a marked repression of the ZEB1 promoter by GRHL2, as did the converse experiment, transfection of the ZEB1 promoter into cells with or without knockdown of endogenous GRHL2 (Fig. 4C).

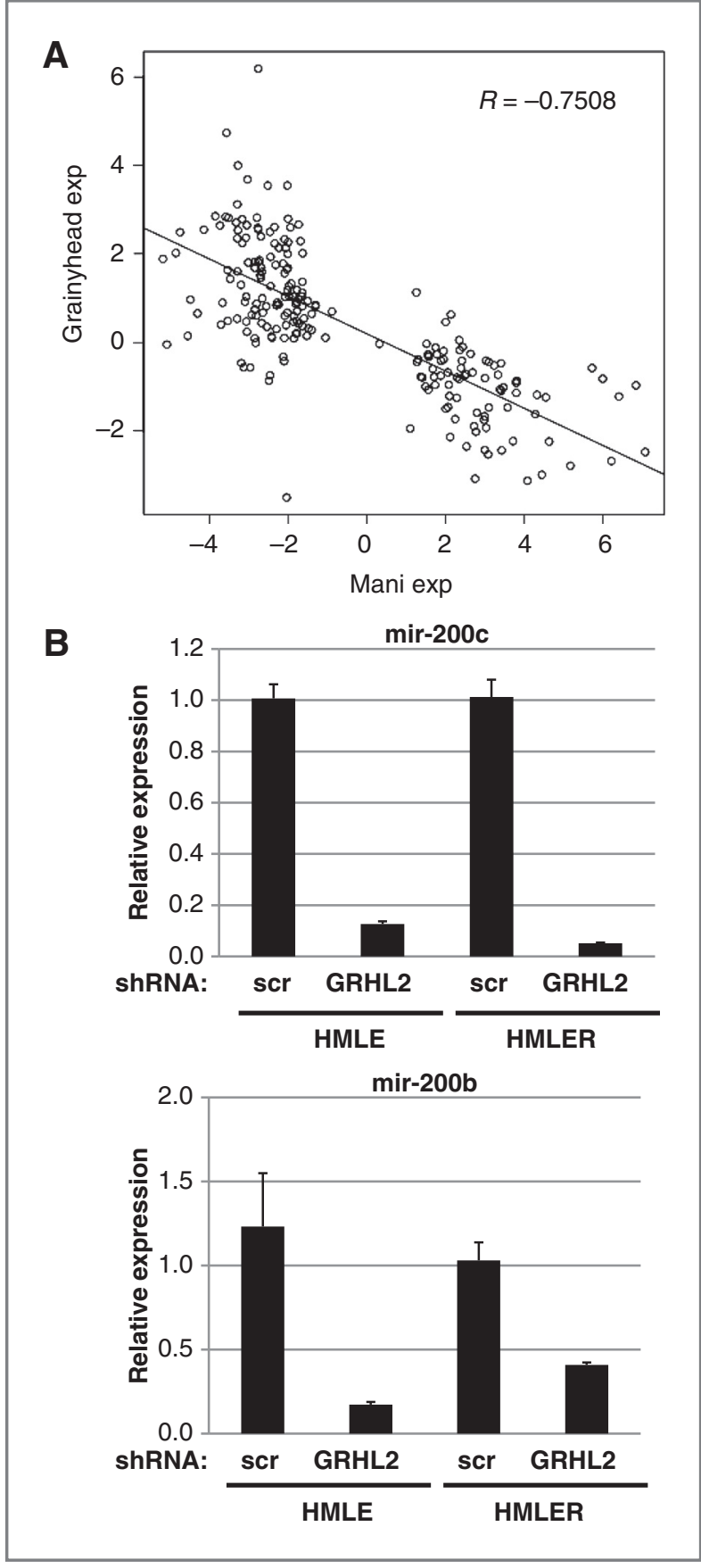

Figure 4. GRHL2 represses the ZEB1 gene. A, HMLE + Twist-ER expressing either empty vector or GRHL2 were induced with 4-OHT for 17 days; 4 days following removal, RNAs were isolated and compared by microarray profiling. Gene changes due to GRHL2 were compared with those due to Twist in the same cell line; a regression plot of this comparison is shown. B, GRHL2 upregulates mir-200b/c. RNAs from HMLE or HMLER cells expressing GRHL2 or control shRNAs were compared for the indicated mir-200 transcripts by RT-PCR.

Inspection of the approximately $1 \mathrm{~kb}$ of promoter sequence that was GRHL2 responsive revealed several potential binding sites for Grainyhead proteins. We tested approximately 200-bp nested fragments of the ZEB1 upstream region, in the context of 


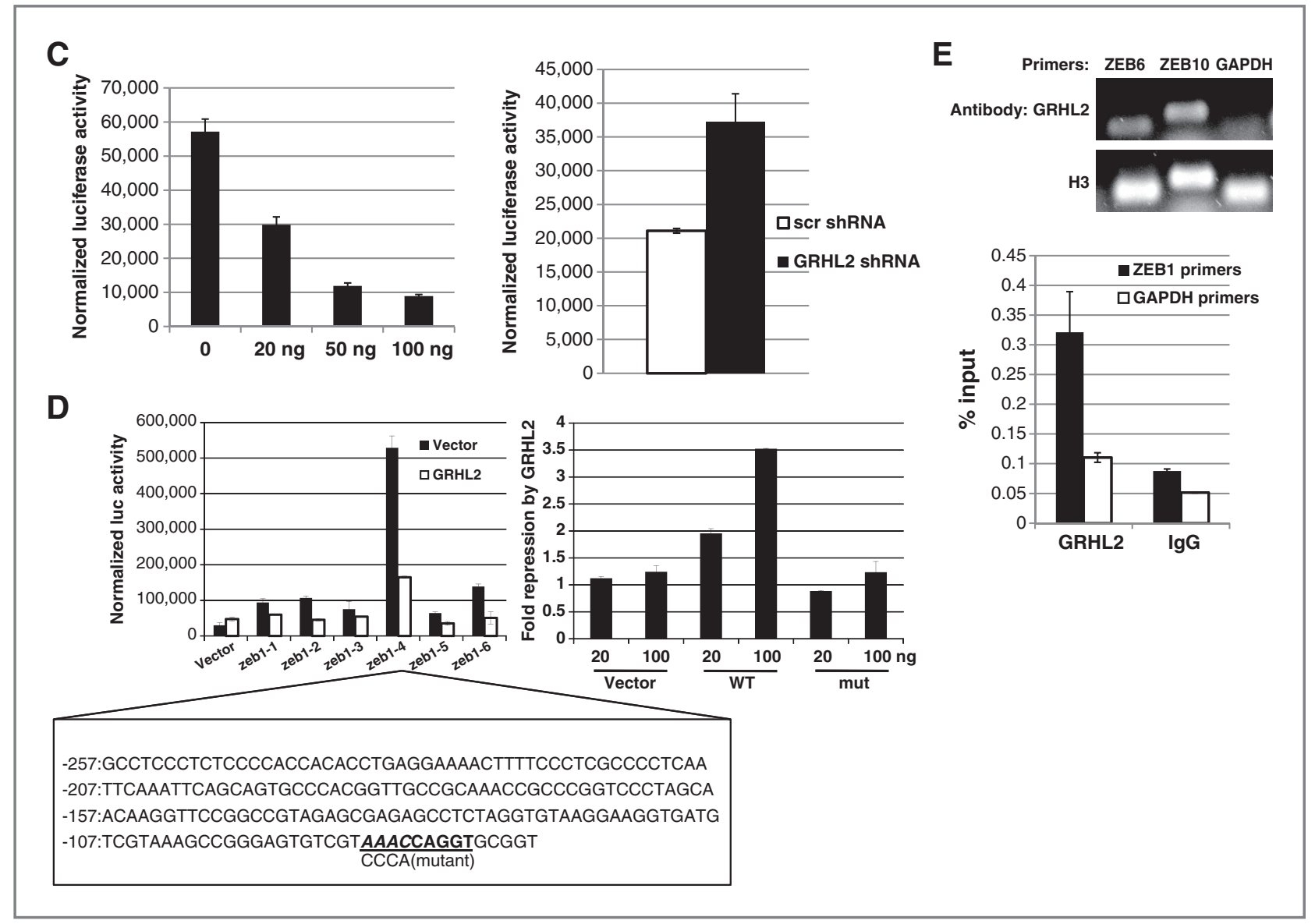

Figure 4. (Continued) C, GRHL2 represses the ZEB1 promoter. Left, MSP cells were cotransfected with a ZEB1 promoter-luciferase reporter construct, with the indicated input amounts of GRHL2 expression vector or equal amounts of empty vector. Values represent the relative luciferase activity normalized to an internal $\beta$-galactosidase control. Right, HMLE + Twist-ER cells (no 4-OHT) with stable GRHL2 knockdown or control (scrambled) knockdown were assayed for luciferase activity after transient transfection of the ZEB1 promoter. D, identification of a ZEB1 promoter fragment that is GRHL2 sensitive and contains a GRHL2 consensus binding site. Left, fragments ( 200 bp) spanning the $1 \mathrm{~kb}$ ZEB1 promoter were assayed for transcriptional activity in the presence or absence of cotransfected GRHL2. The fragment 1 to 4 sequence and predicted GRHL2-binding site are shown. Right, fragment 4 containing wild-type (WT) or mutant (mut) versions of the predicted GRHL2-binding site were assayed for repression by cotransfected GRHL2. E, GRHL2 protein interacts with the ZEB1 promoter. Chromatin from HMLE + Twist-ER + GRHL2 (top) or MDA-MB-231 + GRHL2 (bottom) was immunoprecipitated with GRHL2, histone H3, or nonimmune antibody and analyzed by gel-based PCR or quantitative PCR, using the indicated ChIP primers.

an SV40 promoter, for repression by GRHL2, and identified one fragment (fragment 4) that was highly repressed. This fragment contained a consensus GRHL2-binding site and also carried a strong enhancer; the repression by GRHL2 was completely eliminated by a 4-base mutation of this consensus site (Fig. 4D). To determine whether the ZEB1 promoter was a direct target for repression by GRHL2, ChIP analysis was conducted, showing a strong enrichment of PCR signal using GRHL2 antibody, with respect to nonimmune IgG or a primer set (using GRHL2 antibody) representing an unrelated region of the genome (Fig. 4E). These results indicated that GRHL2 repressed ZEB1 expression and interacted directly with the ZEB1 promoter.

\section{Suppression of ZEB1 is critical for the suppression of EMT by GRHL2}

ZEB1 plays a critical role in EMT in response to various stimuli including TGF- $\beta$ (29-33), informing the hypo- thesis that GRHL2 suppressed EMT, at least in part, by repressing ZEB1 expression. To test this, ZEB1 was expressed ectopically, using a doxycycline-inducible promoter, in the HMLE + Twist-ER + GRHL2 cells. By the criteria of morphology, expression of epithelial and mesenchymal markers, and anoikis resistance, ZEB1 restored EMT that had previously been blocked by GRHL2 expression (Fig. 5A). Analogous effects of ZEB1 expression were also observed in MSP cells that had been reverted to an epithelial phenotype by stable GRHL2 expression (Supplementary Fig. S5). Conversely, in the HMLE cells where GRHL2 knockdown predisposed the cells toward TGF- $\beta$ induced EMT, ZEB1 knockdown blocked this induction (Fig. 5B). Similarly, EMT that was induced by GRHL2 knockdown in HMLER cells was reversed by ZEB1 knockdown (Supplementary Fig. S6). These results indicated that the repression of ZEB1 was a key mechanism by which GRHL2 suppressed EMT. 


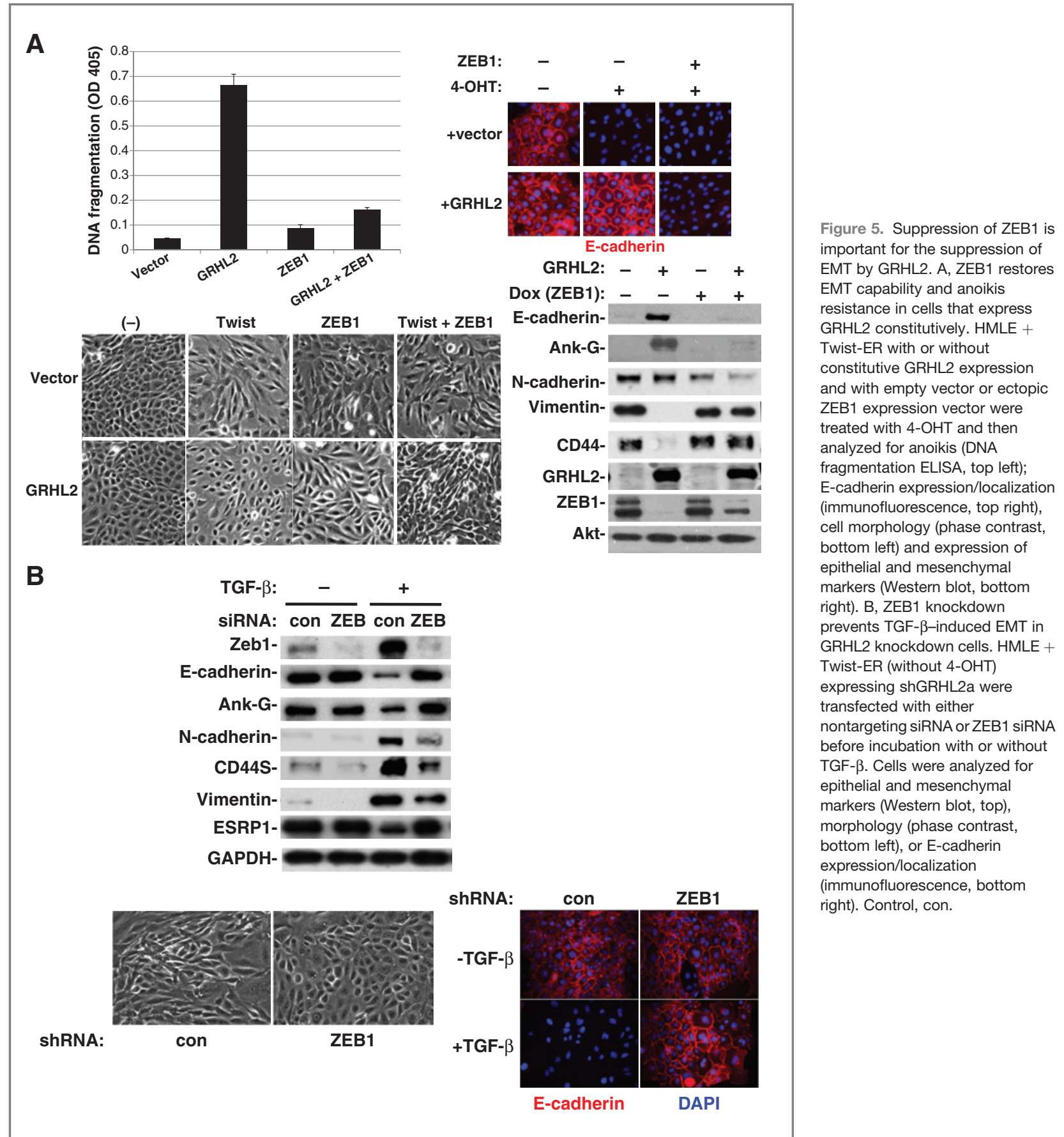

\section{Discussion}

Mammalian GRHL2 is a transcription factor that plays important role in epidermal junctions, in part due to activation of target genes including claudin-4 and E-cadherin. Consistent with this role, the Drosophila Grainyhead gene is among the first transcription factors used in the MZT during embryonic development (16), and the 3 mammalian Grainyhead genes are critical for embryonic and adult wound healing (11-14). In light of the fact that wound healing is orchestrated in part by TGF- $\beta$ signaling (9), the suppressive effect of GRHL2 on this pathway suggests that GRHL2 may contribute to the resolution phase of wound healing, wherein transient EMT-like cell conversions in keratinocytes are instructed to reverse. The suppressive effect of GRHL2 on oncogenic EMT may be understood by analogy to this function, given the similarities between the 2 contexts of EMT (8).

The significance of mammalian Grainyhead proteins in cancer is emerging. GRHL3 was recently shown to function 
as a tumor suppressor in squamous cell carcinoma, acting, at least in part, as a direct activator of PTEN expression (19); EMT-related issues were not examined, however. The GRHL2 gene shows frequent amplification in unclassified breast tumor samples, and has been proposed as a potential oncogene in breast cancer, due, in part, to its suppression of death receptor expression (18). Consistent with this, modest upregulation of GRHL2 mRNA was observed in luminal A, B, and HER2-positive tumor types (Fig. 1D; although it is unclear whether this is an artifactual result of expansion of the epithelial cell compartment relative to normal mammary gland during tumor outgrowth). In contrast, our results show that GRHL2 is downregulated in EMT models and EMT-driven tumor subclasses, and that it suppresses TGF- $\beta$-induced ZEB1 expression; in light of the established protumorigenic potential of ZEB1, this result predicts that GRHL2 will specifically suppress EMT-like tumors $(34,35)$.

These results can be reconciled in light of the diametrically opposed, context-dependent effects of TGF- $\beta$ : growth arrest and tumor suppression in certain tumors versus tumor promotion in others (4). In breast cancer, fewer than $10 \%$ of patients have tumor types (claudin low, metaplastic) in which EMT/TGF- $\beta$ contributes critically to tumor progression, whereas in the majority of tumors (other basal, luminal A, $\mathrm{B}$, and HER2-positive subclasses)-which most transgenic mouse models emulate-TGF- $\beta$ is tumor suppressive $(3,36)$. By targeting the TGF- $\beta$ pathway, GRHL2 is predicted usually to act as an oncogene (i.e., in the most common subclasses of breast cancer) or, less frequently, as a tumor suppressor gene (i.e., in EMT-like subclasses).

The results here indicate that GRHL2 interferes with the response to TGF- $\beta$ by at least 2 mechanisms, interference with Smad2/3-mediated transcriptional activation and direct repression of the ZEB1 promoter (diagrammed in Fig. 6). Consistent with previous observations in other systems $(28,37)$, ZEB1 was required for EMT in response to Twist, TGF- $\beta$, and spontaneous conversion. GRHL2 also upregulated mir-200b/c, consistent with a critical role of the established ZEB1/mir-200 feed-forward regulatory loop in EMT (28). The precise mechanism by which GRHL2 represses the ZEB1 promoter may relate to Grainyhead proteins' ability to repress transcription, by recruiting polycomb repression complex components or by interfering with the binding of a transactivator $(16,38)$.

The mechanism by which GRHL2 inhibits Smad-mediated transcription is unresolved at present. Previous work has shown that ZEB1 protein can bind to the Smad2/3 complex, enhancing transactivation (39); our preliminary observations indicated that this mechanism did not apply in our system. Smad2/3 nuclear versus cytoplasmic localization is regulated by phosphorylation as well as signaling from the Crumbs polarity complex through Hippo pathway components (26). These mechanisms were, however, unlikely to explain the suppression by GRHL2, because Smad phosphorylation and nuclear translocation were not apparently affected. Other nuclear proteins that affect $\operatorname{Smad} 2 / 3$ transactivation, such as TGIF, Ski, and Sno (4), remain to be tested in the context of GRHL2.

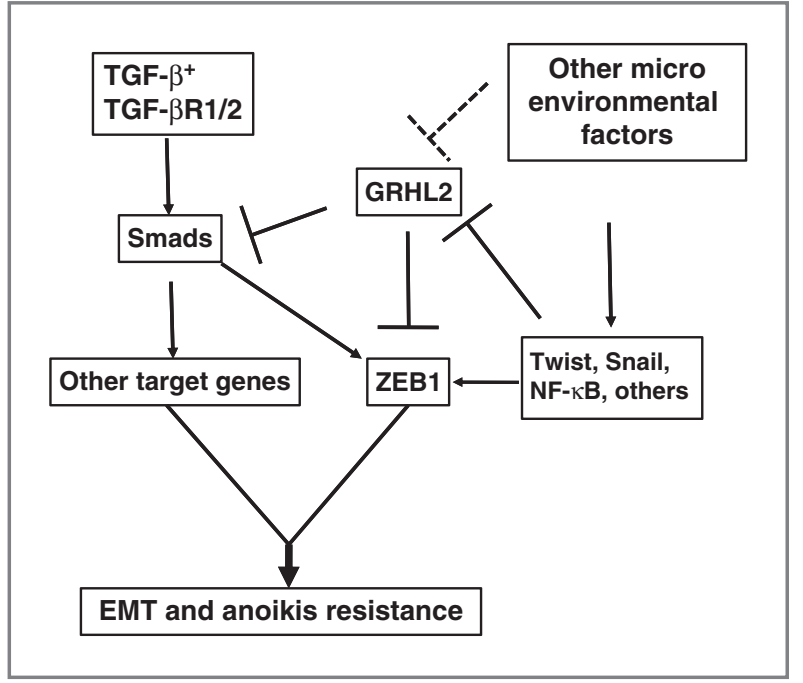

Figure 6. Summary of the proposed model. EMT is induced by the combination of TGF- $\beta$ with other microenvironmental factors and requires the activation of ZEB1 plus other target genes.

Microenvironmental factors (Wnt, NF- $\mathrm{kB}$ agonists?) upregulate Twist and Snail genes, which downregulate GRHL2. This downregulation alleviates the repression of the ZEB1 promoter, permitting TGF- $\beta$ (partly, through Twist and Snail themselves) to activate ZEB1 expression. The downregulation of GRHL2 also enhances Smad-mediated transactivation of TGF- $\beta$ arget genes, which, together with ZEB1, induce EMT and anoikis resistance.

TGF- $\beta$-induced EMT is a highly restricted phenomenon in cell culture models, occurring in only a small number of epithelial cell lines (40). In fact, we observed that the mouse mammary epithelial cell line NMuMg, commonly used to study this phenomenon, has undetectable GRHL2 expression, whereas other mouse mammary lines that are unresponsive do express GRHL2 (Supplementary Fig. S7). These results are consistent with the previous finding that additional factors from the tumor microenvironment confer TGF- $\beta$ responsiveness upon HMLE cells, suggesting that one or more of these factors could function by downregulating GRHL2 (7). More generally, the GRHL2 expression profile in breast cancer samples and cell lines indicate that GRHL2 is a general barrier to EMT. Accordingly, GRHL2 prevented TGF- $\beta$ from conferring anoikis resistance, mammosphere formation (a tumor-initiating cell behavior), and invasive growth in $3 \mathrm{D}$ culture, predicting a tumor-suppressive effect in this context.

These results also suggest that GRHL2 may be a useful biomarker for tumors predicted to respond to TGF- $\beta$ receptor inhibitory drugs currently in clinical trials (41): GRHL2-null tumors, being susceptible to the tumor-promoting effects of TGF- $\beta$, are predicted to respond specifically to this class of drugs, an approach that could improve their efficacy substantially.

\section{Disclosure of Potential Conflicts of Interest \\ No potential conflicts of interest were declared.}

\section{Authors' Contributions}

Conception and design: B. Cieply, A.V. Ivanov, S.M. Frisch

Development of methodology: B. Cieply, A.V. Ivanov, S.M. Frisch 
Acquisition of data (provided animals, acquired and managed patients, provided facilities, etc.): B. Cieply, P. Riley, P.M. Pifer, J. Widmeyer, J. Addison, S.M. Frisch

Analysis and interpretation of data (e.g., statistical analysis, biostatistics, computational analysis): B. Cieply, P.M. Pifer, J. Addison, A.V. Ivanov, J. Denvir, S.M. Frisch

Writing, review, and/or revision of the manuscript: B. Cieply, P. Riley, P.M. Pifer, J. Denvir, S.M. Frisch

Administrative, technical, or material support (i.e., reporting or organizing data, constructing databases): B. Cieply, P. Riley, S.M. Frisch Study supervision: B. Cieply, S.M. Frisch

\section{Acknowledgments}

The authors thank Kathy Brundage (flow cytometry), Kimmi Alonge, and the laboratories of Peter Stoilov, Mike Ruppert, Laura Gibson, Antonio Garcia de Herreros, Antonio Postigo, Richard Myers, and Kai-Schmidt-Ott for help, advice,

\section{References}

1. Polyak K, Weinberg RA. Transitions between epithelial and mesenchymal states: acquisition of malignant and stem cell traits. Nat Rev Cancer 2009;9:265-73.

2. Thiery JP, Acloque H, Huang RY, Nieto MA. Epithelial-mesenchymal transitions in development and disease. Cell 2009;139:871-90.

3. Hennessy BT, Gonzalez-Angulo AM, Stemke-Hale K, Gilcrease MZ, Krishnamurthy S, Lee JS, et al. Characterization of a naturally occurring breast cancer subset enriched in epithelial-to-mesenchymal transition and stem cell characteristics. Cancer Res 2009;69: 4116-24.

4. Massague J. TGFbeta in Cancer. Cell 2008;134:215-30.

5. Taylor MA, Parvani JG, Schiemann WP. The pathophysiology of epithelial-mesenchymal transition induced by transforming growth factor-beta in normal and malignant mammary epithelial cells. J Mammary Gland Biol Neoplasia 2010;15:169-90.

6. Shipitsin M, Campbell LL, Argani P, Weremowicz S, Bloushtain-Qimron N, Yao J, et al. Molecular definition of breast tumor heterogeneity. Cancer Cell 2007;11:259-73

7. Scheel C, Eaton EN, Li SH, Chaffer CL, Reinhardt F, Kah KJ, et al. Paracrine and autocrine signals induce and maintain mesenchymal and stem cell states in the breast. Cell 2011;145:926-40.

8. Schafer M, Werner S. Cancer as an overhealing wound: an old hypothesis revisited. Nat Rev Mol Cell Biol 2008;9:628-38.

9. Margadant C, Sonnenberg A. Integrin-TGF-beta crosstalk in fibrosis, cancer and wound healing. EMBO Rep 2010;11:97-105.

10. Kalluri R. EMT: when epithelial cells decide to become mesenchymallike cells. J Clin Invest 2009;119:1417-9.

11. Ting SB, Caddy J, Hislop N, Wilanowski T, Auden A, Zhao LL, et al. A homolog of Drosophila grainy head is essential for epidermal integrity in mice. Science 2005;308:411-3.

12. Boglev $Y$, Wilanowski T, Caddy J, Parekh V, Auden A, Darido C, et al. The unique and cooperative roles of the Grainy head-like transcription factors in epidermal development reflect unexpected target gene specificity. Dev Biol 2011;349:512-22.

13. Pyrgaki C, Liu A, Niswander L. Grainyhead-like 2 regulates neural tube closure and adhesion molecule expression during neural fold fusion. Dev Biol 2011;353:38-49.

14. Werth M, Walentin $K$, Aue A, Schönheit J, Wuebken A, Pode-Shakked $\mathrm{N}$, et al. The transcription factor grainyhead-like 2 regulates the molecular composition of the epithelial apical junctional complex. Development 2010;137:3835-45.

15. Wilanowski T, Caddy J, Ting SB, Hislop NR, Cerruti L, Auden A, et al. Perturbed desmosomal cadherin expression in grainy head-like 1-null mice. EMBO J 2008;27:886-97.

16. Harrison MM, Botchan MR, Cline TW. Grainyhead and Zelda compete for binding to the promoters of the earliest-expressed Drosophila genes. Dev Biol 2010;345:248-55.

17. Chen W, Dong Q, Shin KH, Kim RH, Oh JE, Park NH, et al. Grainyheadlike 2 enhances the human telomerase reverse transcriptase gene expression by inhibiting DNA methylation at the $5^{\prime}-\mathrm{CpG}$ island in normal human keratinocytes. J Biol Chem 2010;285:40852-63. and reagents and Wioletta Szeszel-Fedorowicz for carrying out the microarray experiment.

\section{Grant Support}

S.M. Frisch was supported by NIH grant R01CA123359. The flow cytometr core facility (Mary Babb Randolph Cancer Center) was supported by NIH grants RR020866 and P20 RR16440. J. Denvir was supported in part by the West Virginia IDEA Network of Biomedical Research Excellence (INBRE) P20 RR 016477-12 (Rankin, Gary, PI). A.V. Ivanov was supported by NIH grant P20 RR16440, American Cancer Society grant 122300-IRG-09-061-04-IRG, and Susan G. Komen for the Cure grant KG110350.

The costs of publication of this article were defrayed in part by the payment of page charges. This article must therefore be hereby marked advertisement in accordance with 18 U.S.C. Section 1734 solely to indicate this fact.

Received December 19, 2011; revised February 6, 2012; accepted February 10 2012; published OnlineFirst February 29, 2012

18. Dompe N, Rivers CS, Li L, Cordes S, Schwickart M, Punnoose EA, et al A whole-genome RNAi screen identifies an $8 q 22$ gene cluster that inhibits death receptor-mediated apoptosis. Proc Natl Acad Sci U S A 2011;108:E943-51.

19. Darido C, Georgy SR, Wilanowski T, Dworkin S, Auden A, Zhao Q, et al Targeting of the Tumor Suppressor GRHL3 by a miR-21-Dependent Proto-Oncogenic Network Results in PTEN Loss and Tumorigenesis. Cancer Cell 2011;20:635-48.

20. Debnath J, Walker SJ, Brugge JS. Akt activation disrupts mammary acinar architecture and enhances proliferation in an mTOR-dependent manner. J Cell Biol 2003;163:315-26.

21. Mani SA, Guo W, Liao MJ, Eaton EN, Ayyanan A, Zhou AY, et al. The epithelial-mesenchymal transition generates cells with properties of stem cells. Cell 2008;133:704-15.

22. Dave N, Guaita-Esteruelas S, Gutarra S, Frias À, Beltran M, Peiró S, et al. Functional cooperation between Snail1 and twist in the regulation of ZEB1 expression during epithelial to mesenchymal transition. J Biol Chem 2011;286:12024-32.

23. Kumar S, Park SH, Cieply B, Schupp J, Killiam E, Zhang F, et al. A pathway for the control of anoikis sensitivity by E-cadherin and epithelial-to-mesenchymal transition. Mol Cell Biol 2011;31:4036-51.

24. Rifat $Y$, Parekh V, Wilanowski T, Hislop NR, Auden A, Ting SB, et al Regional neural tube closure defined by the Grainy head-like transcription factors. Dev Biol 2010;345:237-45.

25. Creighton CJ, Li X, Landis M, Dixon JM, Neumeister VM, Sjolund A et al. Residual breast cancers after conventional therapy display mesenchymal as well as tumor-initiating features. Proc Natl Acad Sci U S A 2009;106:13820-5.

26. Varelas $X$, Samavarchi-Tehrani $P$, Narimatsu $M$, Weiss $A$, Cockburn $K$ Larsen BG, et al. The Crumbs complex couples cell density sensing to Hippo-dependent control of the TGF-beta-SMAD pathway. Dev Cell 2010;19:831-44.

27. Gregory PA, Bracken CP, Smith E, Bert AG, Wright JA, Roslan S, et al An autocrine TGF-beta/ZEB/miR-200 signaling network regulates establishment and maintenance of epithelial-mesenchymal transition. Mol Biol Cell 2011;22:1686-98.

28. Brabletz S, Brabletz T. The ZEB/miR-200 feedback loop-a motor of cellular plasticity in development and cancer? EMBO Rep 2010; 11:670-7.

29. Guaita S, Puig I, Franci C, Garrido M, Dominguez D, Batlle E, et al. Snail induction of epithelial to mesenchymal transition in tumor cells is accompanied by MUC1 repression and ZEB1 expression. J Biol Chem 2002;277:39209-16.

30. Taube JH, Herschkowitz JI, Komurov K, Zhou AY, Gupta S, Yang J, et al. Core epithelial-to-mesenchymal transition interactome geneexpression signature is associated with claudin-low and metaplastic breast cancer subtypes. Proc Natl Acad Sci U S A 2010; 107:15449-54.

31. Smit MA, Peeper DS. Zeb1 is required for TrkB-induced epithelialmesenchymal transition, anoikis resistance and metastasis. Oncogene 2011;30:3735-44. 
32. Aigner K, Dampier B, Descovich L, Mikula M, Sultan A, Schreiber M, et al. The transcription factor ZEB1 (deltaEF1) promotes tumour cell dedifferentiation by repressing master regulators of epithelial polarity. Oncogene 2007;26:6979-88.

33. Grooteclaes ML, Frisch SM. Evidence for a function of CtBP in epithelial gene regulation and anoikis. Oncogene 2000;19:3823-8.

34. Spaderna S, Schmalhofer O, Wahlbuhl M, Dimmler A, Bauer K, Sultan $A$, et al. The transcriptional repressor ZEB1 promotes metastasis and loss of cell polarity in cancer. Cancer Res 2008;68:537-44.

35. Wellner U, Schubert J, Burk UC, Schmalhofer O, Zhu F, Sonntag A, et al. The EMT-activator ZEB1 promotes tumorigenicity by repressing stemness-inhibiting microRNAs. Nat Cell Biol 2009;11:1487-95.

36. Herschkowitz JI, Simin K, Weigman VJ, Mikaelian I, Usary J, Hu Z, et al. Identification of conserved gene expression features between murine mammary carcinoma models and human breast tumors. Genome Biol 2007;8:R76.

37. Schmalhofer O, Brabletz S, Brabletz T. E-cadherin, beta-catenin, and ZEB1 in malignant progression of cancer. Cancer Metastasis Rev 2009;28:151-66.
38. Tuckfield A, Clouston DR, Wilanowski TM, Zhao LL, Cunningham JM, Jane SM. Binding of the RING polycomb proteins to specific target genes in complex with the grainyhead-like family of developmental transcription factors. Mol Cell Biol 2002;22: 1936-46.

39. Postigo AA, Depp JL, Taylor JJ, Kroll KL. Regulation of Smad signaling through a differential recruitment of coactivators and corepressors by ZEB proteins. EMBO J 2003;22:2453-62.

40. Brown KA, Aakre ME, Gorska AE, Price JO, Eltom SE, Pietenpol JA, et al. Induction by transforming growth factor-beta1 of epithelial to mesenchymal transition is a rare event in vitro. Breast Cancer Res 2004;6:R215-31.

41. Ganapathy V, Ge R, Grazioli A, Xie W, Banach-Petrosky W, Kang Y, et al. Targeting the Transforming Growth Factor-beta pathway inhibits human basal-like breast cancer metastasis. Mol Cancer 2010;9:122.

42. Neve RM, Chin K, Fridlyand J, Yeh J, Baehner FL, Fevr T, et al. A collection of breast cancer cell lines for the study of functionally distinct cancer subtypes. Cancer Cell 2006;10:515-27. 


\section{Cancer Research}

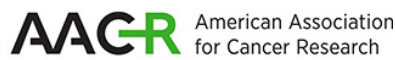

The Journal of Cancer Research (1916-1930) | The American Journal of Cancer (1931-1940)

\section{Suppression of the Epithelial-Mesenchymal Transition by Grainyhead-like-2}

Benjamin Cieply, Philip Riley IV, Phillip M. Pifer, et al.

Cancer Res 2012;72:2440-2453. Published OnlineFirst February 29, 2012.

Updated version Access the most recent version of this article at: doi:10.1158/0008-5472.CAN-11-4038

Supplementary Access the most recent supplemental material at:

Material http://cancerres.aacrjournals.org/content/suppl/2012/02/29/0008-5472.CAN-11-4038.DC1.html

Cited articles This article cites 42 articles, 15 of which you can access for free at: http://cancerres.aacrjournals.org/content/72/9/2440.full.html\#ref-list-1

Citing articles This article has been cited by 24 HighWire-hosted articles. Access the articles at: http://cancerres.aacrjournals.org/content/72/9/2440.full.html\#related-urls

E-mail alerts Sign up to receive free email-alerts related to this article or journal.

Reprints and To order reprints of this article or to subscribe to the journal, contact the AACR Publications Department at Subscriptions pubs@aacr.org.

Permissions To request permission to re-use all or part of this article, contact the AACR Publications Department at permissions@aacr.org. 


\section{Appendix AII}

Farris J., Pifer PM., et al., Grainyhead-like 2 Reverses the Metabolic Changes Induced by the Oncogenic Epithelial-mesenchymal Transition: Effects on Anoikis. Molecular Cancer Research

(In Press) 
Grainyhead-like 2 Reverses the Metabolic Changes Induced by the Oncogenic Epithelialmesenchymal Transition: Effects on Anoikis

Joshua C. Farris ${ }^{1}$, Phillip M. Pifer ${ }^{1}$, Liang Zheng 3 , Eyal Gottlieb ${ }^{3}$, James Denvir ${ }^{4}$, and Steven M. Frisch $^{1,2^{*}}$

1-Mary Babb Randolph Cancer Center

1 Medical Center Drive, Campus Box 9300

West Virginia University

Morgantown, WV 26506

2-Department of Biochemistry

1 Medical Center Drive

West Virginia University

Morgantown, WV 26506

3-Beatson Institute for Cancer Research

Switchback Road

Glasgow, UK

4-Department of Biochemistry and Microbiology

Marshall University

Huntington, West Virginia

*Address for correspondence: Steven M. Frisch, Mary Babb Randolph Cancer Center, 1 Medical Center Drive, Room 2833, West Virginia University, Morgantown, WV 26506. Email:

sfrisch@hsc.wvu.edu

The authors have no conflict of interest to disclose. 


\begin{abstract}
Resistance to anoikis is a pre-requisite for tumor metastasis. The epithelial-to-mesenchymal transition (EMT) allows tumor cells to evade anoikis. The wound healing regulatory transcription factor Grainyhead-like 2 (GRHL2) suppresses/reverses EMT, accompanied by suppression of the cancer stem cell (CSC) phenotype and by re-sensitization to anoikis. Here, the effects of GRHL2 upon intracellular metabolism in the context of reversion of the EMT/CSC phenotype, with a view toward understanding how these effects promote anoikis sensitivity were investigated. EMT enhanced mitochondrial oxidative metabolism. While this was accompanied by higher accumulation of superoxide, the overall level of Reactive Oxygen Species (ROS) declined, due to decreased hydrogen peroxide. Glutamate Dehydrogenase 1 (GLUD1) expression increased in EMT, and this increase, via the product $\alpha$-ketoglutarate $(\alpha-\mathrm{KG})$, was important for suppressing hydrogen peroxide and protecting against anoikis. GRHL2 suppressed GLUD1 gene expression, decreased $\alpha$ KG, increased ROS and sensitized cells to anoikis.
\end{abstract}

Implications: These results demonstrate a mechanistic role for GRHL2 in promoting anoikis through metabolic alterations. 


\section{Introduction}

When deprived of connections to their extracellular matrix, normal epithelial cells trigger a process of programmed cell death referred to as anoikis (1). The ability to metastasize depends critically upon a cancer cell's ability to evade anoikis (2-4). The transcriptional reprogramming event known as the Epithelial to Mesenchymal transition (EMT) confers anoikis resistance and ultimately, increased metastatic potential (5). The increased phenotypic plasticity underlying transitions between epithelial and mesenchymal states is thought to be epigenetically driven (610). One additional manifestation of this increased plasticity, aside from EMT, is that cancer stem cell subpopulations emerge, that, like cells resulting from EMT, are resistant to anoikis; these contribute crucially to both metastasis and disease recurrence.

The wound healing regulatory transcription factor, Grainyhead-like 2 (GRHL2), plays an indispensable role in the maintenance of the epithelial phenotype and branching morphogenesis (11-14). Previously, we reported that GRHL2 suppresses the oncogenic EMT, i.e., promotes MET (15-17). The loss of GRHL2 expression is associated with aggressive, metastatic breast tumor types that contain an unusually large fraction of EMT-like subpopulations, and in the cancer stem cell-like subpopulation of tumors resulting from EMT and/or drug resistance. Interestingly, the constitutive expression of GRHL2 invariably led also to anoikis-sensitivity $(15,16)$. The mechanism underlying this effect was unclear, however.

Reactive oxygen species (ROS) are ubiquitously important in apoptosis through mechanisms such as direct mitochondrial peroxidation of cardiolipin, a lipid which sequesters cytochrome c, as well as direct inactivation of the anti-apoptotic Bcl-2 (18). Accordingly, ROS contributes to anoikis as well as the non-apoptotic cell death of detached cells accompanying ATP loss $(19,20)$. It is unclear at present, however, whether changes in ROS levels occur in EMT or cancer stem cell transitions, and, if so, what drives these changes.

The HMLE cell line is an immortalized mammary epithelial cell line that contains a CD44hi/CD24low subpopulation referred to as the Mesenchymal Sub-Population (MSP) that coexpresses EMT and cancer stem cell phenotypes $(21,22)$. Previously, we reported that MSP cells have low GRHL2 expression, and are resistant to anoikis $(15,16)$. Forced expression of GRHL2 in MSP suppressed their cancer stem cell-like as well as EMT phenotypes and sensitized them to anoikis. The possibility that GRHL2 achieves the latter effect by altering intracellular metabolism has not been explored. 
Glutaminolysis is a critical metabolic pathway on which tumors rely as a major alternate carbon source to glucose, with significant ramifications for cell proliferation, metabolic adaptability and cell survival (23-25). Following deamination of glutamine to produce glutamate, glutamate dehydrogenase-1 (GLUD1 or GDH1) generates the Krebs cycle intermediate $\alpha$-ketoglutarate ( $\alpha$-KG), which is converted by the Krebs cycle to fumarate, an important cofactor for glutathione peroxidase enzymes. Accordingly, $\alpha-\mathrm{KG}$ is an important protective factor against oxidative stress, and GLUD1 is over-expressed in breast and lung carcinomas $(26,27)$.

In this paper, we report that, in reversing EMT, GRHL2 suppresses GLUD1 expression, elevating $\mathrm{H}_{2} \mathrm{O}_{2}$ ROS levels and promoting anoikis-sensitivity. GRHL2 also reversed the cancer stem cell-like shift to oxidative phosphorylation-based ATP production and cell survival. These results inform a novel connection between EMT, metabolic pathways, ROS and anoikis-sensitivity, regulated by GRHL2.

\section{Materials and Methods}

\section{Cell lines}

HMLE, and HMLE+Twist-ER cells and generously provided by R. Weinberg (The Whitehead Institute, Cambridge, MA); MCF10A neoT cells were provided by F. Miller (Karmanos Cancer Center). HMLE and HMLE+Twist-ER cells were maintained in Advanced Dulbecco's Modified Eagle's Medium (DMEM): Ham's F-12 (Gibco) + 5\% horse serum + 1X penicillin-streptomycinglutamine (PSG) $+10 \mu \mathrm{g} / \mathrm{mL}$ insulin, $10 \mathrm{ng} / \mathrm{mL}$ EGF, $0.5 \mu \mathrm{g} / \mathrm{mL}$ hydrocortisone. MCF10A neoT cells were maintained in the same media as HMLE cells with the addition of $0.1 \mu \mathrm{g} / \mathrm{mL}$ cholera toxin. If indicated, HMLE+Twist-ER cells were growth in the presence of 4-hydroxytamoxifen $(10 \mathrm{ng} / \mathrm{mL})$ in order to activate the ER inducible Twist construct. MSP cells were obtained from HMLE cells by sorting for CD44-APC high population/CD24-Cy5.5 LOW. HMLE cells were sorted according to CD24-Cy5.5 high and CD44-APC low.

Generation of stable cell lines by retroviral transduction

The template for Human GRHL2 was purchased from Open Biosystems (MHS442699625903). GRHL2 was subcloned by standard molecular biology protocols into the pMXS-IRESpuro retroviral vector in frame with the C-terminal 3X-FLAG tag (vector contribution of Russ Carstens, University of Pennsylvania). Retroviral packaging and amplification was done in GP2+293T cells by transfection of $4.5 \mu \mathrm{g}$ of retroviral plasmid and $2.5 \mu \mathrm{g}$ of pCMV-VSV-G on 
collagen coated $60 \mathrm{~mm}^{2}$ dishes that were pre-coated for $>1 \mathrm{hr}$ with $50 \mu \mathrm{g} / \mathrm{mL}$ collagen using Lipofectamine 2000 (Invitrogen). Plates were refed 4-6 hours following transfection and viral supernatants were harvested approximately 36 hours following refeed. Viral stocks were filtered through $0.45 \mu \mathrm{m}$ filters (Whatman) and $1 \mathrm{~mL}$ of supernatant was used to infect 1 well of target cells. This was followed by 1,400 RPM centrifugation for 1 hour at room temperature followed by 6 hour or overnight incubation. Infected cells were passaged to $100 \mathrm{~mm}$ dishes, and incubated for 48 hours following infection. Cells were then selected for puromycin $(2 \mu \mathrm{g} / \mathrm{mL}$ for HMLE or MCF10A neoT cells).

Generation of stable cell knockdown cell lines by lentiviral transduction

Lentiviral GLUD1 short hairpin RNA (shRNA) was purchased from Open Biosystems in the pLKO vector. ShRNA \#1 was Open Biosystems catalogue number RHS3979-201758959 and shRNA\#2 was RHS3979-201758962. pLKO scramble control vector was generously contributed by the laboratory of Dr. Scott Weed (WVU). Lentiviral constructs were packaged and amplified in 293T cells by transfection of $3.5 \mu \mathrm{g}$ shRNA vector, $2.3 \mu \mathrm{g}$ sPAX2, and $1.2 \mu \mathrm{g}$ CMV-VSV-G on $60 \mathrm{~mm}^{2}$ dishes that were pre-coated for $>1 \mathrm{hr}$ with $50 \mu \mathrm{g} / \mathrm{mL}$ collagen using Lipofectamine 2000. GRHL2 shRNA was described previously and was shown to duplicate the biologic effects of transfected siRNAs (15).

\section{Western Blotting}

SDS-PAGE was conducted using 4-20\% gradient Tris-Glycine gels (Invitrogen). Proteins were electrophoretically transferred to polyvinylidine difluoride filters (Immobilon) in 5\% methanol Tris-Glycine transfer buffer containing 5\% methanol. PBS + 0.1\% Tween-20 + 5\% nonfat milk were used for blocking filters, primary antibodies were incubated in PBS+0.1\% Tween-20 + $5 \%$ nonfat milk. Primary antibodies were typically incubated between 2 hours at room temperature or overnight at 4 degrees C. Primaries used were: E-cadherin, ms (BD Biosciences), CD44 (HCAM) ms [Santa Cruz Biotech (SCBT)]; GRHL2, rb (Sigma), Akt or pAkt, rb (Cell Signaling); GLUD1, rb (Abcam 168352), $\alpha$-tubulin, Rb (Cell Signaling), Fibronectin, ms (BD Biosciences), GAPDH, ms (Origene), $\beta$-actin, ms (Thermo-pierce), SOD2, Rb (Cell Signaling), HIF1 $\alpha$, ms (BD Biosciences), p110, ms (BD Biosciences), TOMM20, ms (BD Biosciences), VDAC1, Rb (Cell Signaling). Secondary antibodies for chemiluminescence were either anti-mouse or anti-rabbit, conjugated to horseradish peroxidase (HRP) enzyme (Bio-Rad). Secondary antibodies (Biorad) were used at 1:3000 dilution 
and filters were incubated for approximately 1 hour at room temperature. Western blots were developed via ECL Super Signal West Pico (Thermo-Pierce).

\section{Anoikis Assays}

For anoikis assays, caspase activation was measured using the Caspase-Glo 3/7 assay kit (Promega). Cells were dissociated using TrypLE Express (Invitrogen) and a fixed number of cells (1.5 x $10^{5}$ cells) were placed per 6 well poly-(2-hydroxyethyl methacrylate) (poly-HEMA) coated low attachment plates in $2.0 \mathrm{ml}$ of normal growth medium $+0.5 \%$ methylcellulose for the indicated time. Aliquots of cells were mixed 1:1 with caspase glo reagent at indicated time points and assayed for luminescence utilizing a Wallac Envison Perkin Elmer plate reader according to manufacturer's instructions. Where indicated, time-zero cell death values were subtracted from the data presented to normalize for small loading variation.

\section{ROS Assays}

For ROS assay, cells were seeded as above and attached for 48 hours. Cells were detached by trypsinization, and equal numbers of cells were placed in a $15 \mathrm{~mL}$ centrifuge tube. Cells were assayed immediately by flow cytometry after staining for 15 min at 37 degrees with $1 \mathrm{uM} \mathrm{CM-}$ $\mathrm{H}_{2}$ DCFDA (Invitrogen) protected from light. If indicated, $24 \mathrm{hr}$ ROS was examined after plating cells in low attachment polyHEMA ( $2 \mathrm{mg} / \mathrm{mL}$ ) coated $100 \mathrm{~mm}$ dishes in $5 \mathrm{~mL}$ of $0.5 \%$ methylcellulose to prevent excess cell clumping. Cells were harvested by diluting well with $10 \mathrm{~mL}$ DME/F12 + 10\% Horse Serum containing media. Cells were centrifuged at 2000 RPM for 2 minutes. Pellets were

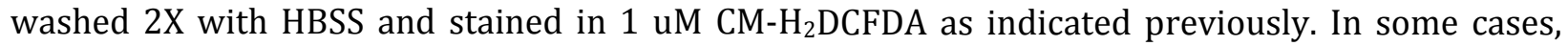
Amplex Red (Invitrogen) assays were used to measure ROS in order to compare attached and suspended conditions and were used according to manufacturer's instructions. For measurement of general cellular superoxide, cells were processed as above and stained with $5 \mathrm{uM}$ dihydroethidium (DHE) (Invitrogen) for 15 minutes at 37 degrees C protected from light followed by flow cytometric analysis at $605 \mathrm{~nm}$ fluorescence. Mitochondrial specific superoxide was measured using mitoSOX (Invitrogen) dye by staining cells with 5 uM mitoSOX solution for 10 minutes at 37 degrees C protected from light. Samples were analyzed by flow cytometry at $580 \mathrm{~nm}$ fluorescence using a BD LSRFortessa Cell analyzer.

\section{ATP Assays}

For the measurement of ATP in attached cells, Cell Titer Glo (Promega) reagent was added to 96 well plates. Luminescence was read after 10 minute incubation at room temperature. Values 
were normalized based on BCA protein assay (Pierce). To measure ATP in detached conditions, cells were detached and placed in 6 well poly-HEMA dishes. Aliquots were mixed 1:1 with Cell Titer Glo reagent. Values were normalized based on BCA protein assay (Pierce).

\section{JC1 Assay}

Cells were seeded on poly-d-lysine coated MatTek dishes. Cells were incubated in $2 \mathrm{ug} / \mathrm{mL}$ JC1 (5',6,6'-tetrachloro-1,1',3,3'-tetraethylbenzimidazolylcarbocyanine iodide) (Invitrogen) at 37 degrees for 30 minutes protected from light. After incubation, dye was washed off of cells and replaced with normal growth medium. Cells were analyzed using an Axiovert 100M LSM510 Zeiss Confocal microscope. Cells were excited with an argon-ion laser source at $488 \mathrm{~nm}$. JC1 monomer emission was observed at $529 \mathrm{~nm}$ and J-aggregate emission was observed at $590 \mathrm{~nm}$. Images were acquired using 20X/0.75 Plan-Neofluar objective. Software used was Zeiss AIM software, version 3.2 .

\section{Oxygen Consumption and Extracellular Acidification Rate}

The oxygen consumption rates (OCR) and extracellular acidification rate (ECAR) of adherent cells were measured utilizing the Seahorse XF Extracellular Flux Analyzer (Seahorse Biosciences Inc., North Billerica, MA). This method of analysis allows for real time monitoring of extracellular acidification rates and oxygen consumption based on two fluorimetric probes inserted into the small volume well. Equal numbers of cells (7500 cells per well) were seeded in a defined small volume in Seahorse XF-96 well plates. The following day, wells were refed with fresh HEPES buffered media approximately 1 hour prior to assay. In short, the sensor cartridge was loaded with metabolic inhibitors to target specific components of the electron transport chain at specific times, while monitoring $\mathrm{pH}$ change as well as oxygen consumption. The metabolic inhibitors used are oligomycin (ATP synthase inhibitor), followed by the protonophore FCCP (an uncoupler of oxidative phosphorylation), and finally a combination of rotenone (complex I inhibitor) and antimycin A (complex III inhibitor). Basal OCR and ECAR measurement during time course treatment with the inhibitors listed above allow for calculations of basal OCR, maximum mitochondrial respiratory capacity, OCR due to proton leak, ATP production, spare respiratory capacity, and non-mitochondrial derived OCR. Fatty acid oxidation study was done by treating cells overnight prior to assay with substrate limited media (DME/F12 media $+1 \%$ horse serum, 0.5 mM carnitine hydrochloride [pH adjusted], 1X PSG, 0.5 mM D-(+)-Glucose, 5 mM HEPES buffer). On day of assay, substrate limited media was replaced with FAO medium 45 minutes prior to the start 
of the assay (Homemade Krebs Henseleit Buffer modified with $\mathrm{CaCl}_{2}$ : To $500 \mathrm{~mL}$ sterile water the following was added: $111 \mathrm{mM} \mathrm{NaCl}, 5.7 \mathrm{mM} \mathrm{KCl}, 1.25 \mathrm{mM} \mathrm{CaCl}_{2}, 2.0 \mathrm{mM} \mathrm{MgSO}$, $1.2 \mathrm{mM} \mathrm{Na}_{2} \mathrm{HPO}_{4}$; This buffer was supplemented with $2.5 \mathrm{mM}$ glucose, $0.5 \mathrm{mM}$ carnitine, $5 \mathrm{mM}$ HEPES, $\mathrm{pH}$ adjusted to 7.4 with $\mathrm{NaOH}$ ). 15 minutes prior to start of assay, $40 \mu \mathrm{M}$ Etomoxir was added to the appropriate wells. At the beginning of the assay, either palmitate:BSA or BSA control was added to appropriate wells. Mitostress test was the conducted according to manufacturer's instructions. For glycolytic stress test, cells are deprived of glucose for approximately 1 hour prior to the beginning of the assay. ECAR is measured and glucose is re-introduced to the media. The increase in ECAR is attributed to glucose conversion to pyruvate through glycolysis. Oligomycin is then added to shunt all metabolic function to glycolysis, which results in a large increase in ECAR indicating the glycolytic reserve. Following addition of 2-deoxy-glucose (competitive inhibitor of hexokinase), the non-glucose derived OCR can be calculated.

\section{Flow Cytometry Sorting}

Trypsinized cells $\left(1 \times 1^{6}\right)$ were stained with APC tagged mouse anti-human CD44 (BD Biosciences 560890), and PerCP-Cy5.5 tagged mouse anti-human CD24 (BD Biosciences 561647) in $100 \mathrm{uL}$ of flow sorting buffer (1X PBS, $2.5 \mathrm{mmol} / \mathrm{L}$ EDTA, $10 \mathrm{mmol} / \mathrm{L}$ HEPES, and 2\% horse serum) on ice protected from light for 30 minutes. Following staining, cells were washed with 1X HBSS and resuspended in flow sorting buffer. Cells were analyzed and sorted using a FACS-Aria.

\section{Cell Culture Antioxidant Treatments}

For suppression of reactive oxygen species, the following antioxidants were used. Where indicated, HMLE cells were treated with N-acetyl cysteine (NAC) at the concentration indicated for $1 \mathrm{hr}$ prior to assay. Reduced glutathione (GSH) was added to HMLE cells after pH correction to 7.4 and incubated for approximately 1-2 hours depending on experiment at the indicated concentration. 6-hydroxy-2,5,7,8-tetramethylchroman-2-carboxylic acid (Trolox) was added to cells at the indicated concentration for overnight pretreatment of HMLE or HMLE+shGLUD1 cells. HMLE or HMLE+shGLUD1 cells were treated with cell permeable di-methyl- $\alpha$-ketoglutarate (Sigma) which was pH corrected to 7.4. Cells were treated at the indicated concentration between overnight and 2 hour pre-treatment.

\section{RNA Sequencing}


RNA was isolated in triplicate from both MSP cells expressing vector alone or stably expressing GRHL2. For RNA isolation, the RNeasy Plus Kit (Qiagen) was used and was quantified using Nanodrop (Fisher Scientific). The RNA quality check was performed on Bioanalyzer (Agilent). RNA quality check and libraries builds were done by the WVU genomics core. RNA samples were then sent to the University of Illinois Core Facility for 100BP single end data RNA sequencing. FastQC was used to visualize quality of sequencing. After it was determined that no filtering was needed, the reads were mapped to hg38 using STAR. The data was then quantified with featureCounts with the following flag set: -ignore dup-primary. This count data was used as input into DEseq2 to produce a list of differentially expressed genes with some visualizations also included. The list of genes with FPKM was also acquired and was produced from the count data with a simple custom script.

\section{Kinetic $\alpha-K G$ assay}

HMLE, MSP, or MSP+GRHL2 cells were seeded in 6 well dishes in duplicate wells and allowed to attach for 24 hours. Wells were washed 2X with PBS, followed by lysis in 300 uL RIPA

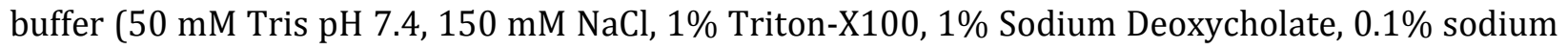
dodecyl sulfate, + pierce miniprotease inhibitor table per $10 \mathrm{~mL}$ lysis buffer). Lysates were scraped into microfuge tubes and cleared in cold centrifuge at $4^{\circ} \mathrm{C}$ at full speed for 10 minutes. Supernatants were saved and assayed in $100 \mathrm{uL}$ reaction buffer $\left(100 \mathrm{mM} \mathrm{KPO}_{4} \mathrm{pH} 7.2,10 \mathrm{mM} \mathrm{NH}_{4} \mathrm{Cl}, 5 \mathrm{mM}\right.$ $\mathrm{MgCl}_{2}$, and $0.15 \mathrm{mM} \mathrm{NADH}$ added immediately prior to assay). $10 \mathrm{uL}$ of sample were added to 100 $\mathrm{uL}$ reaction buffer. Immediately prior to the beginning of the assay, $0.7 \mathrm{uL}(0.5$ units) of LGlutamate Dehydrogenase enzyme (bovine liver, sigma G2626) was added to each of the wells using a multi-channel pipettor. Positive control samples were done to ensure linear range of $\alpha$-KG concentration in samples. OD was read kinetically at $37^{\circ} \mathrm{C}$ at $340 \mathrm{~nm}$ reading the sample every 10 20 seconds depending on the number of samples in the assay. Depending on the level of $\alpha$-KG in the sample, the reaction would proceed more quickly or more slowly. The slope of the line created by monitoring the decline in NADH absorbance as it was used up in the reaction was calculated and indicated the relative concentration of $\alpha$-KG. Calculated slopes were normalized to protein in the samples as determined quantitatively by BCA assay (Pierce).

\section{Extraction of Cells for LC-MS Analysis}

All solvents used were high quality HPLC gradient grade and sterile milliQ grade water was used. Cells were plated in triplicate per condition in 6 well dishes. Cells were seeded for at least 24 
hours prior to extraction. Wells were washed 3X with PBS. Extract solution was made up of 50\% methanol, 30\% acetonitrile, and 20\% milliQ water. Extraction buffer was pre-chilled in a dry ice ethanol bath. Eppendorf tubes were pre-chilled in a dry ice/ethanol bath placed in a metal heating block for maximum cooling. Intracellular extracts were made by adding $500 \mathrm{uL}$ of pre-chilled extraction buffer to wells and placing plate on a shaker in the cold room for 10 minutes. The solution was then transferred into pre-chilled Eppendorf tubes. Samples were centrifuged at $0{ }^{\circ} \mathrm{C}$ at full speed for 10 minutes. Supernatants were transferred to HPLC vials and stored at $-80 \stackrel{\circ}{\circ}$. For LCMS method, Column was the sequant Zic-pHilic $(150 \mathrm{~mm} \times 2.1 \mathrm{~mm}$ i.d. $5 \mu \mathrm{m})$ with the guard column (20 mm $\times 2.1 \mathrm{~mm}$ i.d. $5 \mu \mathrm{m}$ ) from HiChrom, Reading, UK. Mobile phase A: $20 \mathrm{mM}$ ammonium carbonate plus $0.1 \%$ ammonia hydroxide in water. Mobile phase B: acetonitrile. The flow rate was kept at $100 \mu \mathrm{min}^{-1}$ and gradient as follow: $0 \mathrm{~min} 80 \%$ of $\mathrm{B}, 30 \mathrm{~min} 20 \%$ of $\mathrm{B}, 31 \mathrm{~min} 80 \%$ of $\mathrm{B}, 45 \mathrm{~min} 80 \%$ of $\mathrm{B}$. The mass spectrometer (Thermo Exactive Orbitrap) was operated in a polarity switching mode.

\section{Immunofluorescence}

Cells were plated on chamber slides (LabTek154526) using $\sim 0.5 \mathrm{ml}$ medium per chamber. Wells were pre-coated with sterile $0.5 \mathrm{mg} / \mathrm{ml}$ poly-lysine or $20 \mathrm{ug} / \mathrm{ml}$ collagen for $\sim 1$ hour, and washed off prior to plating. Cells were washed in HBSS or D-PBS, and fixed in EM grade 4\% formaldehyde in 1X PBS for 20 minutes at room temperature. Cells were washed 2X with PBS. Samples were then permeabilized in $0.5 \%$ TX100 in PBS at 4 degrees for 10 minutes. Permeabilization solution was washed 2X with PBS. Cells were incubated in 100mM glycine in PBS for 10 minutes to quench the formaldehyde. Cells were blocked for one hour in PBS+10\% goat serum $+0.1 \%$ Tween-20+0.1\%BSA for 1 hour. Primary antibody was diluted in blocking solution, and incubated $1 \mathrm{hr} / \mathrm{RT}$ or overnight/4 degrees (TOMM20, ms; BD Biosciences). Primary antibody was typically diluted 1:250. After probing, cells were washed with PBS+0.1\% Tween-20, 3X. Secondary antibody (Anti-mouse Alex Fluor 555; Invitrogen) was diluted in blocking solution, and incubated 1-2 hr/RT (typically 1:1000 dilution). Cells were washed again with PBS+0.1\% Tween20, 3X. Samples mounted in ProLong Gold+DAPI.

\section{Statistical Analysis}

Error bars in graphs represent SDs. P-values were calculated using a Student's two-tailed ttest.

\section{Results}


EMT shifts metabolism from glycolysis to oxidative phosphorylation, which is reversed by GRHL2.

MSP and TGF- $\beta$-induced MCF10aneoT cells result from EMT that requires GRHL2 downregulation $(15,16,28)$, accompanied by a CD44high CD24low marker profile (figure $1 \mathrm{~A})$. Reexpression of GRHL2 sensitized these cell lines to anoikis significantly, as assessed by a caspase activation assay (figure 1B, 1C, figure S1A). Conversely, the stable knockdown of GRHL2 using shRNA conferred anoikis-resistance in HMLE+Twist-ER cells as well as MCF10A neoT cells (figure 1D, S1B).

We first endeavored to characterize the effect of EMT and, conversely, the effect of GRHL2induced MET on metabolism in our system. We found that the ATP level in attached MSP cells was elevated significantly compared to HMLE cells. When cells were suspended, ATP level declined significantly in HMLE cells, but was maintained in MSP cells, even at twenty-four hours of suspension (Figure 2A). GRHL2 reduced the level of ATP in both suspended and attached MSP cells (figure 2B).

In light of the higher efficiency of mitochondrial oxidative phosphorylation compared to glycolysis in the production of ATP, these results suggested that EMT perhaps caused a shift to the former. MSP cells had a higher mitochondrial membrane potential $(\Delta \Psi)$, reflecting increased oxidative phosphorylation. Conversely, GRHL2 overexpression resulted in decreased $\Delta \Psi$ in MSP cells (Figure 2C). These results, and the marked ATP differences, indicated that EMT shifted cells toward an increased utilization of mitochondrial oxidative phosphorylation to increase basal ATP levels and sustain ATP even in suspended cells.

In order to test the effect of EMT or GRHL2-induced MET on regulation of oxidative metabolism specifically, we compared oxygen consumption rate in HMLE, MSP, and MSP+GRHL2 cells using the Seahorse mitochondrial stress test. The Seahorse XF assay system measures extracellular acidification rate (ECAR) as well as oxygen consumption rate (OCR) in order to determine fluxes in metabolic parameters that occur upon addition of various inhibitors and stimulators of the electron transport chain. Our results indicated that MSP cells had greater ATP, maximum respiration, as well as spare oxidative capacity relative to HMLE cells, and re-expression of GRHL2 reversed these effects (figures 3A,B). The increased mitochondrial activity of MSP and reversal by GRHL2 were not limited to cells utilizing glucose and glutamine as carbon sources, as similar effects were seen with the fatty acid carnitine as the sole carbon source (figures S4A,S4B). Moreover, HMLE cells had higher glycolytic activity than MSP cells (figure S2A). Stably depleting 
GRHL2 in both HMLE+Twist-ER and MCF10A neoT cells partially reversed the mesenchymal metabolic phenotype, increasing ATP and maximum respiration (figure S2B, S2C, S2D). To determine whether the increased OCR observed was due to elevated mitochondrial number, cell lines were stained for a mitochondrial structural protein, TOMM20. Surprisingly, we found that MSP cells had lower mitochondrial staining than HMLE cells (figure S2E, S2F). This may indicate that HMLE cells partially compensate for low mitochondrial activity by up-regulating mitochondrial biogenesis. Taken together, these results confirmed that mesenchymal cells enhanced oxidative phosphorylation, and partially suppressed glycolysis, both of which are counteracted by GRHL2. Confirming this observation, we found that treatment with 2-deoxyglucose (2-DG) resulted in greater decrease in ATP level in HMLE cells (58\% of no treatment control) and MSP+GRHL2 cells (59.1\% of no treatment control) than in MSP cells (72.1\% of no treatment control) (figure S3A). GLUD1 depletion did not directly affect ATP levels, as reported (29). The loss of GLUD1 did, however, result in a decrease in mitochondrial membrane potential (figure S3B).

\section{EMT suppresses the accumulation of ROS; reversal by GRHL2}

To identify genes regulated by GRHL2 underlying its metabolic effects, we conducted RNA-seq analysis. Surprisingly, we did not find that major ETC components or Krebs cycle metabolic enzymes were significantly affected by GRHL2 expression, with the possible exception of pyruvate dehydrogenase kinase 4 (PDK4), but its levels were highly sensitive to the initial conditions of each individual experiment, precluding definitive conclusions as to its regulation (data not shown). GRHL2 did, however, regulate several genes that participate in reactive oxygen species (ROS) regulation (Table 1). Interestingly, GRHL2 expression did not significantly affect expression of the major ROS regulatory enzymes superoxide dismutase or catalase (Table 1).

We compared endogenous ROS levels in HMLE, MSP, and MSP+GRHL2 cells. We found that the MSP cells display a significant reduction in ROS by fluorigenic reporter assay (CM- $\left.\mathrm{H}_{2} \mathrm{DCFDA}\right)$ in both 0 hour and 24 hour suspended conditions, and that GRHL2 increased ROS to the approximate level detected in HMLE cells (figure 4A). Inducing EMT in the basal mammary epithelial cell line, MCF10A neoT, with TGF- $\beta$ treatment resulted in decreased ROS; further, overexpression of GRHL2 elevated ROS level (figure S5A). Conversely, shRNA depletion of GRHL2 suppressed ROS levels both in HMLE+Twist-ER cells and MCF10A neoT cells (figures 4C, S5D).

To identify the species of ROS present, we analyzed cells with a variety of fluorometric dyes with varying species selectivities. CM- $\mathrm{H}_{2}$ DCFDA staining is a general ROS indicator that responds 
well to hydrogen peroxide $\left(\mathrm{H}_{2} \mathrm{O}_{2}\right)$. In order to verify that this was the predominant species, we stained cells with MitoSOX red, dihydroethidium (DHE), and Amplex red-hydrogen peroxide reporter. Amplex Red, which reports hydrogen peroxide specifically, indicated ROS trends similar to those obtained by $\mathrm{CM}-\mathrm{H}_{2}$ DCFDA staining in both attached and suspended conditions (figure 4B). MitoSOX red and DHE staining report mitochondrial and generalized superoxide, respectively. These probes showed that HMLE cells had lower mitochondrial and general superoxide $\left(\mathrm{O}_{2^{-}}\right)$levels than MSP cells and that overexpression of GRHL2 resulted in a decreased $\mathrm{O}_{2}$ - level (figure S5B, S5C). Mitochondrial electron transport is the major source of cellular superoxide (30). In this light, our superoxide data correlated well with our data on mitochondrial oxidative phosphorylation. These data indicated clearly that mitochondria are not the major source of higher ROS in normal epithelial cells or GRHL2-reverted epithelial cells, as this ROS was hydrogen peroxide, not superoxide, and mitochondria were more active in mesenchymal cells, which had lower overall ROS.

We further sought to examine the role played by ROS in contributing to anoikis sensitivity. HMLE cells were treated with two common antioxidant compounds including reduced glutathione and the vitamin E derivative, Trolox. We found that these compounds reduced ROS level in cells and significantly reduced both ROS and sensitivity to anoikis (Figure 4D). In figure S5A, we show that MCF10a neoT cells experience a large ROS burst between 0 and 24 hours. Speculatively, this burst may occur much earlier in HMLE cells, resulting in little difference between 0 and 24 hours. It is also plausible that in HMLE cells at 24 hours, enough cell death has occurred to obscure the ROS burst. These findings support the crucial role of ROS in triggering anoikis.

We have previously reported that CD44S, which is upregulated in MSP cells, contributes to anoikis resistance (31). CD44 was shown elsewhere to decrease ROS by shifting metabolism from oxidative phosphorylation toward glycolysis and pentose phosphate pathways, thereby increasing reduced glutathione levels (32). Consistent with this, the stable knockdown of CD44 resulted in increased ROS, and increased sensitivity to anoikis (figure S7A, S7B). Our metabolic findings were inconsistent with the mechanism proposed in (32) because we observed a shift toward oxidative phosphorylation in MSP cells (figure 3) and because reduced glutathione levels in HMLE and MSP cells were similar (figure S8).

Interestingly, neutralizing cellular ROS in HMLE cells with the scavenger N-acetyl cysteine (NAC) resulted in a significant increase in $\Delta \Psi$ (Figure 4E). Neutralizing ROS in MSP+GRHL2 cells replicated this effect (figure S6A), indicating that the low-ROS cellular environment could play a role in sustaining the elevated mitochondrial function that we observed in mesenchymal cells (figure 2). 
EMT up-regulates glutamate dehydrogenase-1; reversal by GRHL2

Our RNA-seq data indicated that GRHL2 down-regulated several genes that have well established effects on ROS in other systems (Table 1). Although NQO1 knockdown potentiated anoikis in non-small cell cancer cells (33), manipulation of the levels of NCF2/p67PHOX, ALOX15, FAT4 and NQO1 produced no significant change in ROS in our cells (data not shown). GRHL2 also down-regulated Glutamate Dehydrogenase 1 (GDH1 or GLUD1), an enzyme implicated in the suppression of ROS (27). By western blotting, we found that GLUD1 protein was upregulated in MSP cells relative to HMLE cells, and downregulated in MSP by GRHL2 expression, in agreement with our RNA-seq data (figure 5A). Similarly, EMT that was generated by transient activation of Twist-ER protein also up-regulated GLUD1, which was prevented by GRHL2 (figure 5B); similar effects were found in TGF- $\beta$-induced MCF10a neoT cells (Figure S8). Conversely, GLUD1 expression increased following depletion of GRHL2 in both HMLE+Twist-ER and MCF10A neoT cells (figure 5C), indicating that GRHL2 is an important repressor of GLUD1.

Depletion of GLUD1 was previously shown to reduce $\alpha$-ketoglutarate $(\alpha-K G)$ levels, indicating that GLUD1, through glutaminolysis, is a major source of $\alpha$-KG (27). Interestingly, we found that MSP cells have an elevated $\alpha$-KG:succinate ratio relative to HMLE cells and that GRHL2 decreased $\alpha-K G$, using both enzymatic and liquid chromatography/mass spectrometric assays, consistent with the expression levels of GLUD1 (Figure 5D, 5E, 5F).

\section{GLUD1 suppresses ROS and anoikis}

Next, we tested the effects of altered GLUD1 and $\alpha$-KG levels on ROS and anoikis. The stable knockdown of GLUD1 enzyme using shRNA transduction resulted in both an increase in ROS level as well as increased sensitivity to anoikis (figure 6A). A similar effect of GLUD1 knockdown was observed in HMLE+Twist-ER cells (figure S8C).

To confirm that the GLUD1 effect on ROS was mediated by $\alpha$-KG, we tested the effect of $\alpha$ KG supplementation using the cell-permeable di-methyl- $\alpha$-ketoglutarate derivative in GLUD1depleted cells. This resulted in a dose dependent protection from anoikis as well as a reduction in ROS in HMLE cells (figure 6B). Moreover, the excess anoikis observed in HMLE cells as well as 
MSP+GRHL2 cells was suppressed by $\alpha$-KG replacement, demonstrating that the loss of GLUD1 promoted anoikis through ROS (figure 6C, 6D, S10). Neutralizing ROS with antioxidants or $\alpha$-KG partially protected HMLE+shGLUD1 cells against anoikis as well (figure 6E, 6F). These data indicate the critical role of GLUD1 up-regulation during EMT, resulting in protection from anoikis by suppressing ROS, and the converse role of GRHL2 in promoting anoikis through GLUD1 downregulation (summarized in figure 7).

\section{Discussion}

The role and mechanism of the Warburg-like shift to aerobic glycolysis in tumor cells has received extensive investigation, but this effect is now known to be highly context dependent. In fact, recent studies indicate that cancer stem cells (related, in some instances, to EMT) develop an enhanced capacity for and dependence on oxidative phosphorylation ((34),see (35) for an excellent review). Moreover, oxidative phosphorylation can contribute to tumor metastasis $(36,37)$. This is thought, counter-intuitively, to have advantages for slow-cycling cancer stem cells, such as better maintenance of ATP in nutrient-poor tumor environments due to the higher efficiency of the TCA/ETC pathway (using fatty acids or glutamine as carbon sources); moreover, the tumor microenvironment is rarely, if ever, hypoxic enough to inhibit oxidative phosphorylation (35). Our metabolic results comparing MSP vs. HMLE cells are highly consistent with this new understanding, and they show that GRHL2 reverts MSP cells back to an epithelial type metabolism (higher glycolysis, lower oxidative phosphorylation), accompanying re-sensitization to anoikis.

Metabolic changes due to oncogenic transformation, EMT or transition to cancer stem cells have been reported extensively, with unique effects in each system $(36,38-41)$. In this paper, we provide a mechanism by which EMT/cancer stem cell transition lowers ROS so at protect cells against anoikis. Under this model, GRHL2 reverses EMT, suppressing mitochondrial oxidative function by repression of the critical enzyme regulating glutaminolysis, GLUD1, resulting in loss of glutamine utilization, increased ROS level, ultimately shifting metabolism resulting in anoikis sensitivity (Figure 6).

There may be multiple mechanisms of ROS neutralization downstream of $\alpha$-ketoglutarate. First, it can act synergistically with other compounds (e.g., ascorbic acid) as a co-antioxidant (42). Secondly, the reverse GLUD1 reaction yields glutamate, contributing to glutathione synthesis. Thirdly, $\alpha$-ketoglutarate is a co-factor for both histone demethylase and DNA demethylase enzymes. Changes in the level of this co-factor could affect gene expression epigenetically, as observed in 
other settings (43), with effects on metabolism and ROS. Finally, a recent report shows that upregulated GLUD1 in tumor cells led to increases in $\alpha$-ketoglutarate, increasing fumarate, in turn, through Krebs cycle enzymes. This contributed to the neutralization of cellular ROS, because fumarate activated glutathione peroxidase activity. GLUD1 up-regulation was, accordingly, shown to be pro-tumorigenic, and this effect was mediated by decreased ROS levels (27). In renal cell carcinoma, fumarate hydratase (FH) deficiency was increased fumarate to millimolar levels, contributing to oxidative stress rather than alleviating it (44). However, the major ROS in that study was superoxide rather than hydrogen peroxide, and GRHL2 did not affect FH level in our cells (data not shown). We speculate that the contradictory effects of fumarate in FH-deficient vs. GLUD1-over-expressing tumor cells may be reconciled by these considerations.

Interestingly, autophagy, which may play positive or negative roles in anoikis and nonapoptotic cell death after detachment, is regulated by glutaminolysis as well, through mTORC complexes (25). Speculatively, GLUD1/ $\alpha$-ketoglutarate alterations due to EMT or GRHL2 may affect cell survival through these additional pathways.

The upregulation of GLUD1 would have multiple advantages for EMT/CSC cells, including increased use of glutamine or glutamate as alternative carbon sources in glucose-poor environments, protection against ROS, and the increased ETC/oxidative phosphorylation capability afforded by less ROS-induced mitochondrial damage. Interestingly, the aspartate/glutamate transporter SLC1A6 was down-regulated dramatically by EMT, and, conversely, up-regulated by GRHL2 (table 1). Recently, SLC1A6 was found to act primarily as a glutamate exporter (45). EMT/CSC cells are predicted, therefore, to have increased accumulation of intracellular glutamate, contributing to the pathway that we have emphasized here.

\section{Acknowledgments}

The authors thank Dr. Stephanie Rellick and the laboratory of Dr. James Simpkins for assistance with Seahorse techniques, Dr. Kathy Brundage for flow cytometry, Drs. John Hollander and John LeMasters for technical advice concerning mitochondrial metabolism, Tyler Calkins for early contributions to the project, Aniello Infante and Ryan Percifield for assistance in RNA library construction and analysis of RNA seq data, Dr. Zachary Schafer for providing constructs and technical advice, Dr. Michael Ochs for significant guidance with bioinformatics analysis, and Dr. Amanda Ammer for expertise in Confocal image acquisition. The work was supported by a grant from the Mary Kay Foundation and a grant from the National Institute Of General Medical Sciences, 
U54GM104942. The following NIH grants supported the flow cytometry facility: GM103488/RR032138; RR020866;OD016165;GM103434.

\section{References}

1. Frisch SM, Francis H. Disruption of epithelial cell-matrix interactions induces apoptosis. J Cell Biol. 1994;124:619-26.

2. Chiarugi P, Giannoni E. Anoikis: a necessary death program for anchorage-dependent cells. Biochem Pharmacol. 2008;76:1352-64.

3. Paoli P, Giannoni E, Chiarugi P. Anoikis molecular pathways and its role in cancer progression. Biochim Biophys Acta. 2013;1833:3481-98.

4. Buchheit CL, Weigel KJ, Schafer ZT. Cancer cell survival during detachment from the ECM: multiple barriers to tumour progression. Nat Rev Cancer. 2014.

5. Frisch SM, Schaller M, Cieply B. Mechanisms that link the oncogenic epithelial-mesenchymal transition to suppression of anoikis. J Cell Sci. 2013;126:21-9.

6. Malouf GG, Taube JH, Lu Y, Roysarkar T, Panjarian S, Estecio MR, et al. Architecture of epigenetic reprogramming following Twist1-mediated epithelial-mesenchymal transition. Genome biology. 2013;14:R144.

7. Sharma SV, Lee DY, Li B, Quinlan MP, Takahashi F, Maheswaran S, et al. A chromatinmediated reversible drug-tolerant state in cancer cell subpopulations. Cell. 2010;141:69-80.

8. Singh B, Shamsnia A, Raythatha MR, Milligan RD, Cady AM, Madan S, et al. Highly adaptable triple-negative breast cancer cells as a functional model for testing anticancer agents. PloS one. 2014;9:e109487.

9. Javaid S, Zhang J, Anderssen E, Black JC, Wittner BS, Tajima K, et al. Dynamic chromatin modification sustains epithelial-mesenchymal transition following inducible expression of Snail-1. Cell reports. 2013;5:1679-89.

10. McDonald OG, Wu H, Timp W, Doi A, Feinberg AP. Genome-scale epigenetic reprogramming during epithelial-to-mesenchymal transition. Nature structural \& molecular biology. 2011;18:86774.

11. Walentin K, Hinze C, Werth M, Haase N, Varma S, Morell R, et al. A Grhl2-dependent gene network controls trophoblast branching morphogenesis. Development. 2015;142:1125-36.

12. Senga K, Mostov KE, Mitaka T, Miyajima A, Tanimizu N. Grainyhead-like 2 regulates epithelial morphogenesis by establishing functional tight junctions through the organization of a molecular network among claudin3, claudin4, and Rab25. Mol Biol Cell.23:2845-55.

13. Werth M, Walentin K, Aue A, Schonheit J, Wuebken A, Pode-Shakked N, et al. The transcription factor grainyhead-like 2 regulates the molecular composition of the epithelial apical junctional complex. Development.137:3835-45.

14. Gao X, Vockley CM, Pauli F, Newberry KM, Xue Y, Randell SH, et al. Evidence for multiple roles for grainyhead-like 2 in the establishment and maintenance of human mucociliary airway epithelium.[corrected]. Proceedings of the National Academy of Sciences of the United States of America. 2013;110:9356-61.

15. Cieply B, Riley Pt, Pifer PM, Widmeyer J, Addison JB, Ivanov AV, et al. Suppression of the Epithelial-Mesenchymal Transition by Grainyhead-like-2. Cancer research. 2012;72:2440-53.

16. Cieply B, Farris J, Denvir J, Ford HL, Frisch SM. Epithelial-Mesenchymal Transition and Tumor Suppression Are Controlled by a Reciprocal Feedback Loop between ZEB1 and Grainyheadlike-2. Cancer research. 2013;73:6299-309. 
17. Mlacki M, Kikulska A, Krzywinska E, Pawlak M, Wilanowski T. Recent discoveries concerning the involvement of transcription factors from the Grainyhead-like family in cancer. Experimental biology and medicine. 2015;240:1396-401.

18. Huttemann M, Pecina P, Rainbolt M, Sanderson TH, Kagan VE, Samavati L, et al. The multiple functions of cytochrome $\mathrm{c}$ and their regulation in life and death decisions of the mammalian cell: From respiration to apoptosis. Mitochondrion. 2011;11:369-81.

19. Schafer ZT, Grassian AR, Song L, Jiang Z, Gerhart-Hines Z, Irie HY, et al. Antioxidant and oncogene rescue of metabolic defects caused by loss of matrix attachment. Nature. 2009;461:10913.

20. Kamarajugadda S, Stemboroski L, Cai Q, Simpson NE, Nayak S, Tan M, et al. Glucose oxidation modulates anoikis and tumor metastasis. Molecular and cellular biology. 2012;32:1893907.

21. Scheel C, Eaton EN, Li SH, Chaffer CL, Reinhardt F, Kah KJ, et al. Paracrine and autocrine signals induce and maintain mesenchymal and stem cell States in the breast. Cell. 2011;145:926-40. 22. Mani SA, Guo W, Liao MJ, Eaton EN, Ayyanan A, Zhou AY, et al. The epithelial-mesenchymal transition generates cells with properties of stem cells. Cell. 2008;133:704-15.

23. DeBerardinis RJ, Mancuso A, Daikhin E, Nissim I, Yudkoff M, Wehrli S, et al. Beyond aerobic glycolysis: transformed cells can engage in glutamine metabolism that exceeds the requirement for protein and nucleotide synthesis. Proceedings of the National Academy of Sciences of the United States of America. 2007;104:19345-50.

24. Lu W, Pelicano H, Huang P. Cancer metabolism: is glutamine sweeter than glucose? Cancer cell. 2010;18:199-200.

25. Villar VH, Merhi F, Djavaheri-Mergny M, Duran RV. Glutaminolysis and autophagy in cancer. Autophagy. 2015;11:1198-208.

26. Friday E, Oliver R, 3rd, Welbourne T, Turturro F. Glutaminolysis and glycolysis regulation by troglitazone in breast cancer cells: Relationship to mitochondrial membrane potential. Journal of cellular physiology. 2011;226:511-9.

27. Jin L, Li D, Alesi GN, Fan J, Kang HB, Lu Z, et al. Glutamate dehydrogenase 1 signals through antioxidant glutathione peroxidase 1 to regulate redox homeostasis and tumor growth. Cancer cell. 2015;27:257-70.

28. Werner S, Frey S, Riethdorf S, Schulze C, Alawi M, Kling L, et al. Dual roles of the transcription factor grainyhead-like 2 (GRHL2) in breast cancer. The Journal of biological chemistry. 2013;288:22993-3008.

29. Kang SW, Lee S, Lee EK. ROS and energy metabolism in cancer cells: alliance for fast growth. Arch Pharm Res. 2015;38:338-45.

30. Mailloux RJ, Harper ME. Mitochondrial proticity and ROS signaling: lessons from the uncoupling proteins. Trends in endocrinology and metabolism: TEM. 2012;23:451-8.

31. Cieply B, Koontz C, Frisch SM. CD44S-hyaluronan interactions protect cells resulting from EMT against anoikis. Matrix biology : journal of the International Society for Matrix Biology. 2015;48:55-65.

32. Tamada M, Nagano O, Tateyama S, Ohmura M, Yae T, Ishimoto T, et al. Modulation of glucose metabolism by CD44 contributes to antioxidant status and drug resistance in cancer cells. Cancer research. 2012;72:1438-48.

33. Madajewski B, Boatman MA, Chakrabarti G, Boothman DA, Bey EA. Depleting Tumor-NQ01 Potentiates Anoikis and Inhibits Growth of NSCLC. Molecular cancer research : MCR. 2016;14:1425.

34. Gordon N, Skinner AM, Pommier RF, Schillace RV, O'Neill S, Peckham JL, et al. Gene expression signatures of breast cancer stem and progenitor cells do not exhibit features of Warburg metabolism. Stem cell research \& therapy. 2015;6:157. 
35. Viale A, Corti D, Draetta GF. Tumors and Mitochondrial Respiration: A Neglected Connection. Cancer research. 2015.

36. LeBleu VS, O'Connell JT, Gonzalez Herrera KN, Wikman H, Pantel K, Haigis MC, et al. PGC1alpha mediates mitochondrial biogenesis and oxidative phosphorylation in cancer cells to promote metastasis. Nature cell biology. 2014;16:992-1003, 1-15.

37. Amoedo ND, Rodrigues MF, Rumjanek FD. Mitochondria: are mitochondria accessory to metastasis? Int J Biochem Cell Biol. 2014;51:53-7.

38. Cuyas E, Corominas-Faja B, Menendez JA. The nutritional phenome of EMT-induced cancer stem-like cells. Oncotarget. 2014;5:3970-82.

39. Dong C, Yuan T, Wu Y, Wang Y, Fan TW, Miriyala S, et al. Loss of FBP1 by Snail-mediated repression provides metabolic advantages in basal-like breast cancer. Cancer cell. 2013;23:316-31. 40. Vlashi E, Lagadec C, Vergnes L, Reue K, Frohnen P, Chan M, et al. Metabolic differences in breast cancer stem cells and differentiated progeny. Breast cancer research and treatment. 2014;146:525-34.

41. Birsoy K, Possemato R, Lorbeer FK, Bayraktar EC, Thiru P, Yucel B, et al. Metabolic determinants of cancer cell sensitivity to glucose limitation and biguanides. Nature. 2014;508:10812.

42. Kang YH, Park SH, Lee YJ, Kang JS, Kang IJ, Shin HK, et al. Antioxidant alpha-keto-carboxylate pyruvate protects low-density lipoprotein and atherogenic macrophages. Free radical research. 2002;36:905-14.

43. Carey BW, Finley LW, Cross JR, Allis CD, Thompson CB. Intracellular alpha-ketoglutarate maintains the pluripotency of embryonic stem cells. Nature. 2015;518:413-6.

44. Zheng L, Cardaci S, Jerby L, MacKenzie ED, Sciacovelli M, Johnson TI, et al. Fumarate induces redox-dependent senescence by modifying glutathione metabolism. Nature communications. 2015;6:6001.

45. Beaudin S, Welsh J. 1,25-Dihydroxyvitamin D induces the glutamate transporter SLC1A1 and alters glutamate handling in non-transformed mammary cells. Molecular and cellular endocrinology. 2016.

\section{Figure Legends}

Figure 1. MSP cells are an anoikis resistant subpopulation of HMLE cells which have undergone EMT and lost GRHL2 expression. A. (upper panel): HMLE cells were sorted for CD44 and CD24 by FACS (insert shows up-regulated CD44S in MSP cells); B: Resulting sub-populations were assayed for anoikis sensitivity by caspase 3/7 activation assay; C. MSP cells in which GRHL2 was re-expressed were assayed for anoikis compared to vector control (24 hour time point with zero time subtracted). (lower panel): confirmation of GRHL2 expression by western blotting. D. HMLE+Twist-ER cells depleted of GRHL2 by shRNA transduction were assayed for anoikis; the confirmation of GRHL2 knockdown is shown as part of figure 5C. 
Figure 2. ATP levels are elevated in mesenchymal relative to epithelial cells due to increased mitochondrial membrane potential $(\Delta \Psi)$ A. HMLE and MSP cells were analyzed for ATP level under attached or suspended (16 h, $24 \mathrm{~h}$ ) conditions. (The line indicates that the attached level cannot be directly compared to the suspended levels due to proliferation in attached condition). B. GRHL2 suppresses ATP level in MSP cells in both attached and suspended conditions. C. $\Delta \Psi$ is elevated in MSP cells, which is reduced by GRHL2 overexpression (JC1 fluorescent probe; green=monomers to indicate free JC1, orange=aggregates of JC1 proportional to $\Delta \Psi$ ); (right panel): Image J quantification and ratio calculation of raw intensity pixel density for single phase red and green images.

Figure 3. Shift from glycolysis to oxidative phosphorylation accompanies EMT; reversal by GRHL2 A. MSP cells have higher ATP production, maximum respiration, and higher spare capacity for oxidative phosphorylation than HMLE; B. Reversal by GRHL2

Figure 4. Epithelial cells have higher ROS than mesenchymal cells, and the excess ROS promotes anoikis. A. HMLE cells have higher ROS compared to MSP cells, which is elevated by GRHL2 expression in MSP cells: CM- $\mathrm{H}_{2}$ DCFDA fluorescence at the indicated times of suspension. B. Hydrogen peroxide is the major regulated ROS type (Amplex Red assay); C. shRNA depletion of GRHL2 decreases ROS in HMLE+Twist-ER cells. D. Antioxidant compounds protect against anoikis. MSP+GRHL2 cells were pre-treated overnight with reduced glutathione (GSH, $5 \mathrm{mM}$ ) and Trolox (100 uM) followed by ROS and anoikis assay; E. Suppression of ROS resulted in increased JC1 aggregate formation and elevated $\Delta \Psi$. HMLE cells were treated with $\mathrm{N}$-acetyl cysteine (NAC) at 0.25 and $0.5 \mathrm{mM}$ for $1 \mathrm{hr}$ before staining with JC-1 fluorimetric dye. (right panel): Image J quantification and ratio calculation of raw intensity pixel density of single phase red and green images.

FIGURE 5. GRHL2 regulates Glutamate dehydrogenase-1 and $\alpha$-ketoglutarate levels. A, GLUD1 is downregulated by GRHL2 in MSP cells (upper panel: RNA-seq data lower panel: western blot); B. GLUD1 expression is upregulated during EMT and is downregulated by GRHL2 in HMLETwist cells; C. shRNA depletion of GRHL2 results in increased GLUD1 protein. D. $\alpha$-KG is higher in MSP cells relative to HMLE cells, and GRHL2 overexpression decreases it (enzymatic assay measuring loss of NADH absorbance); E. $\alpha$-KG:succinate ratio is higher in MSP than in HMLE or 
MSP+GRHL2 cells (mass spectrometric assay). F. $\alpha$-KG:succinate ratio is decreased by GRHL2 in HMLE cells induced to undergo EMT by Twist-ER activation (mass spectrometric assay).

FIGURE 6. GLUD1 and $\alpha-K G$ regulate anoikis through ROS. A. (upper panel): Western blot analysis of HMLE+shGLUD1 cells. (lower panel): GLUD1 knockdown elevates ROS level; (B) GLUD1 knockdown promotes anoikis as assessed by $\mathrm{CM}-\mathrm{H}_{2}$ DCFDA assay and caspase 3/7 activation assay (respectively); C. Dimethyl- $\alpha$-KG lowers ROS; (D) Dimethyl- $\alpha-K G$ protects HMLE cells against anoikis; E. DM- $\alpha$ KG and Trolox lower ROS level; (F) DM- $\alpha$ KG and Trolox protect GLUD1 knockdown cells against anoikis.

FIGURE 7. GRHL2 suppresses EMT and results in the loss of GLUD1. This decreases $\alpha$-KG, suppressing oxidative metabolism, increasing ROS, and sensitizing cells to anoikis.

Table 1. Effect of GRHL2 on expression of ROS-regulatory genes

\begin{tabular}{|c|c|c|}
\hline Gene ID & $\begin{array}{l}\text { Fold Change } \\
\text { (MSP+GRHL2/ } \\
\text { MSP+vec control) }\end{array}$ & Significance in ROS or Metabolism \\
\hline $\begin{array}{l}\text { ALOX15 } \\
\text { ALOXE3 } \\
\text { ALOX12P2 }\end{array}$ & $\begin{array}{l}+236.0 \\
+21.0 \\
+18.3\end{array}$ & $\begin{array}{l}\text { Can reportedly generate lipid peroxide radicals from } \\
\text { arachidonic acid substrates }\end{array}$ \\
\hline SLC1A6 & +165.7 & $\begin{array}{l}\text { Glutamate/Aspartate transporter which may alter } \alpha-K G D H \\
\text { activity resulting in } \mathrm{H}_{2} \mathrm{O}_{2} \text { radicals and reduce its TCA activity }\end{array}$ \\
\hline $\begin{array}{l}\text { NCF2 (p67- } \\
\text { PHOX) }\end{array}$ & +23.4 & Cytoplasmic subunit of NADPH-Oxidase (NOX) Complex \\
\hline FAT4 & -19.6 & $\begin{array}{l}\text { Human homologue of Drosophila Fat. Potentially contains } \mathrm{Ft}_{\text {mito }} \\
\text { fragment which binds complex I and stabilizes it to reduce } \\
\text { ROS/increase mitochondrial activity }\end{array}$ \\
\hline SOD1 & -1.4 & $\begin{array}{l}\text { Soluble superoxide dismutase enzyme involved in cytoplasmic } \\
\mathrm{O}_{2}-\text { detoxification. Not significantly by GRHL2 overexpression }\end{array}$ \\
\hline
\end{tabular}




\begin{tabular}{|c|c|c|}
\hline SOD2 & +1.2 & $\begin{array}{l}\text { Mitochondrial isoform of superoxide dismutase involved in } \\
\text { conversion of mitochondrial } \mathrm{O}_{2}^{--} \text {into diffusible } \mathrm{H}_{2} \mathrm{O}_{2} \text {. Not } \\
\text { significantly altered by GRHL2 overexpression }\end{array}$ \\
\hline SOD3 & NA & Extracellular superoxide dismutase \\
\hline Cat & -1.3 & $\begin{array}{l}\text { Catalase enzyme involved in detoxification of } \mathrm{H}_{2} \mathrm{O}_{2} \text { ROS species. } \\
\text { Appears to be slightly regulated by EMT, but not significantly } \\
\text { altered by GRHL2 overexpression }\end{array}$ \\
\hline NQO1 & -1.9 & $\begin{array}{l}\text { ROS detoxifying enzyme which reduces quinines to } \\
\text { hydroquinones - major Nrf2 target gene }\end{array}$ \\
\hline GLUD1 & -2.5 & $\begin{array}{l}\text { Reported be the primary source of cellular regulation of } \alpha-K G \\
\text { which feeds to TCA cycle to increase Fumarate and stabilize } \\
\text { GPx1 }\end{array}$ \\
\hline
\end{tabular}


Figure 1

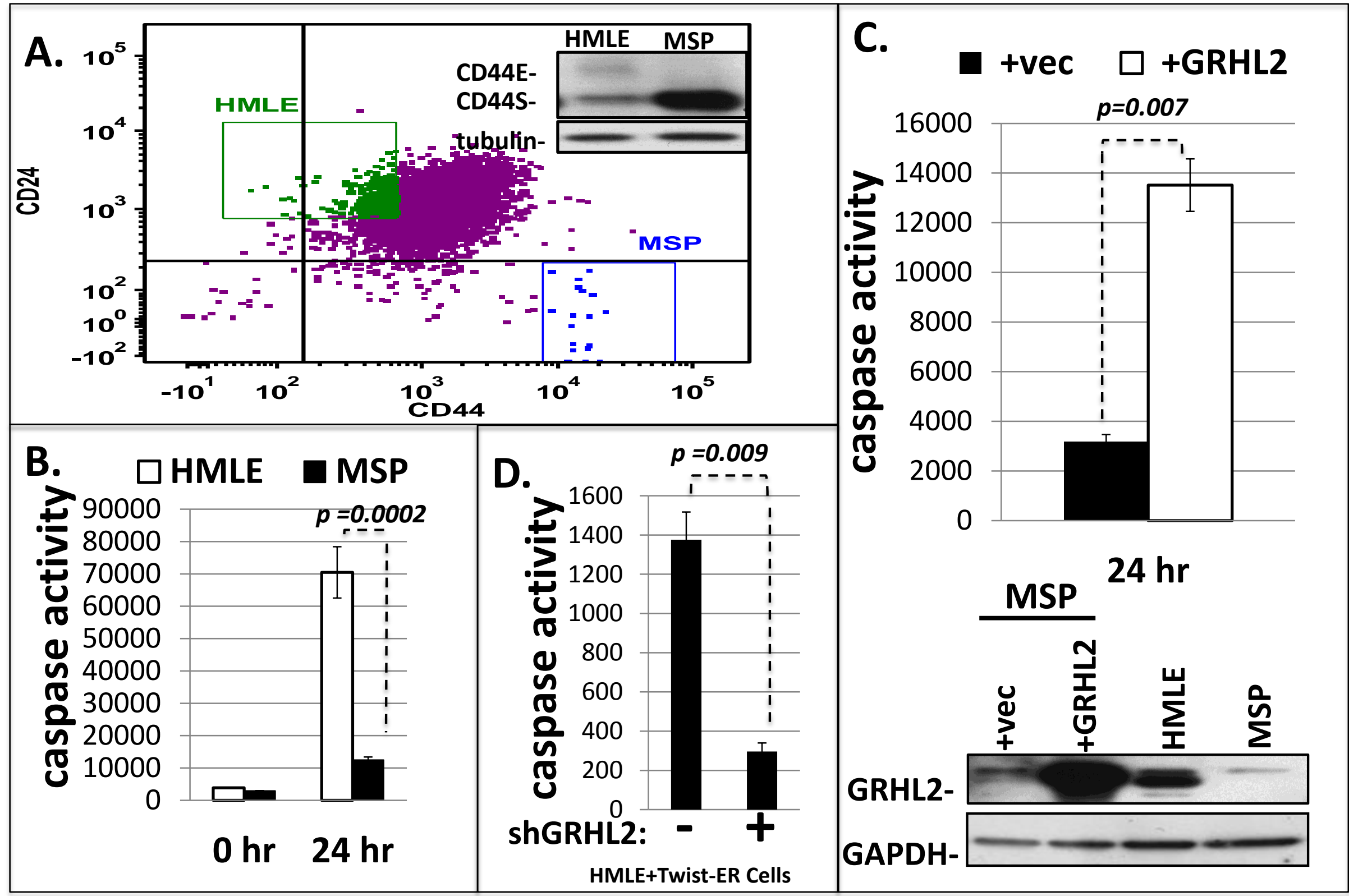


Figure 2

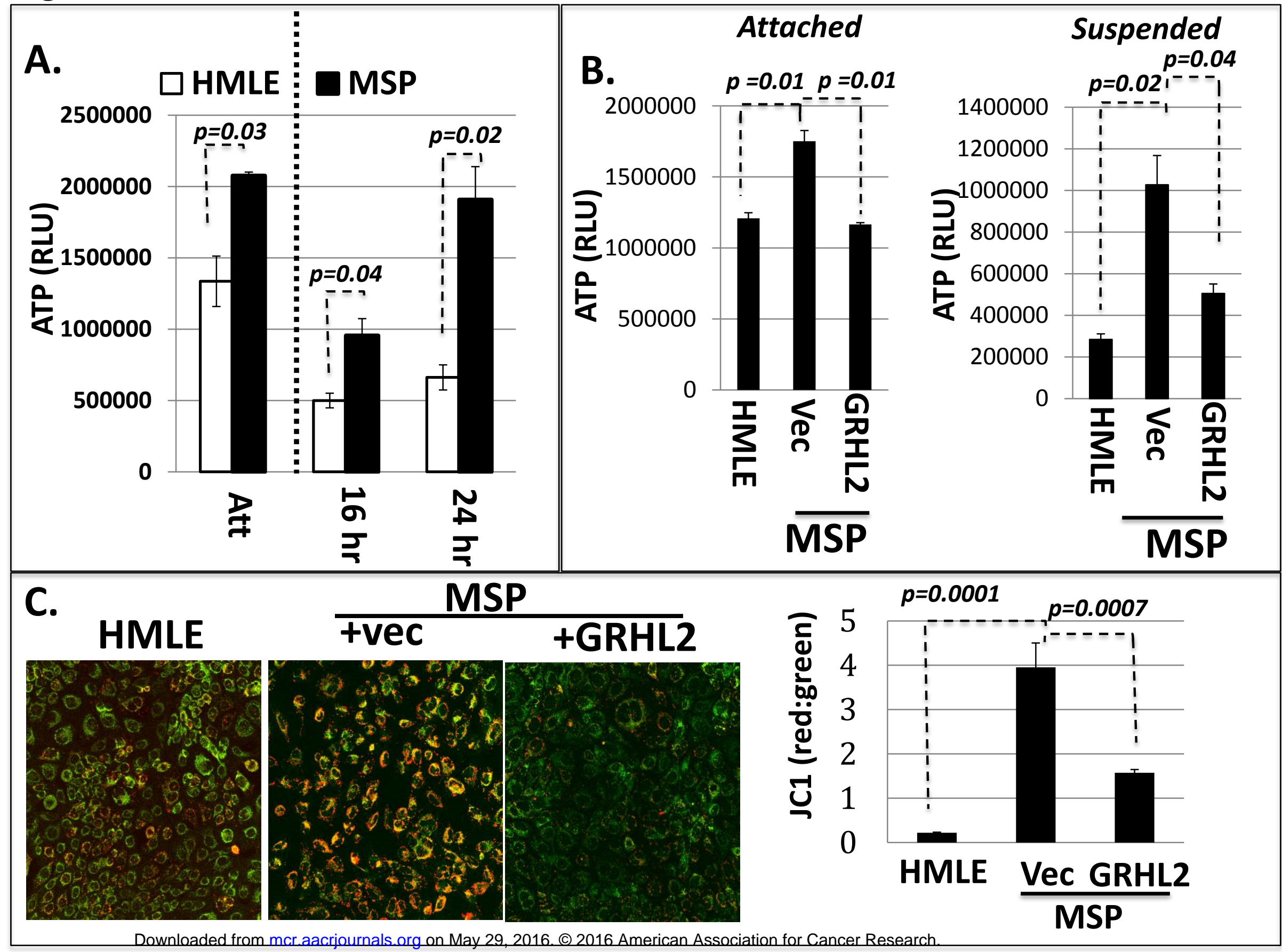


Figure 4

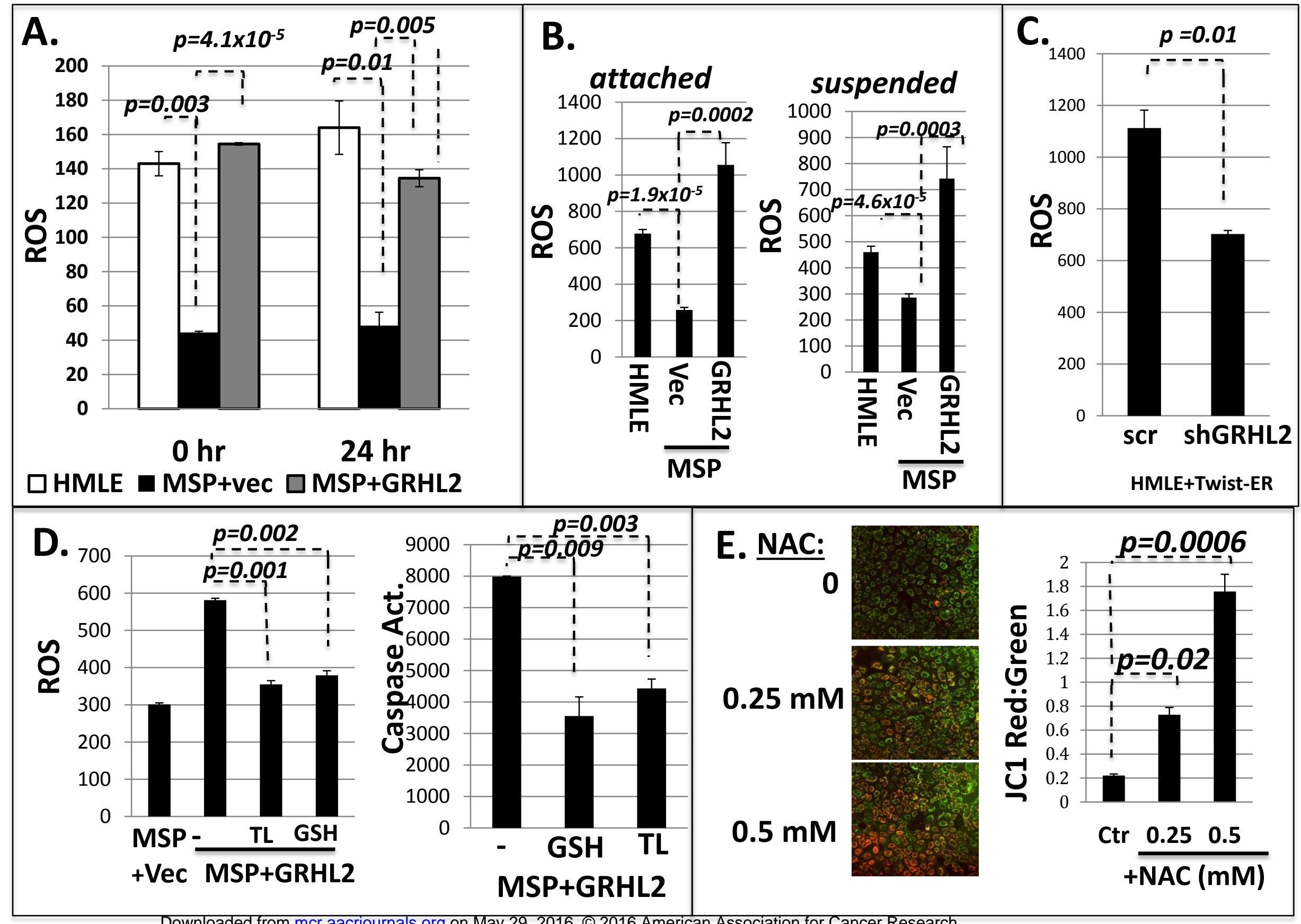




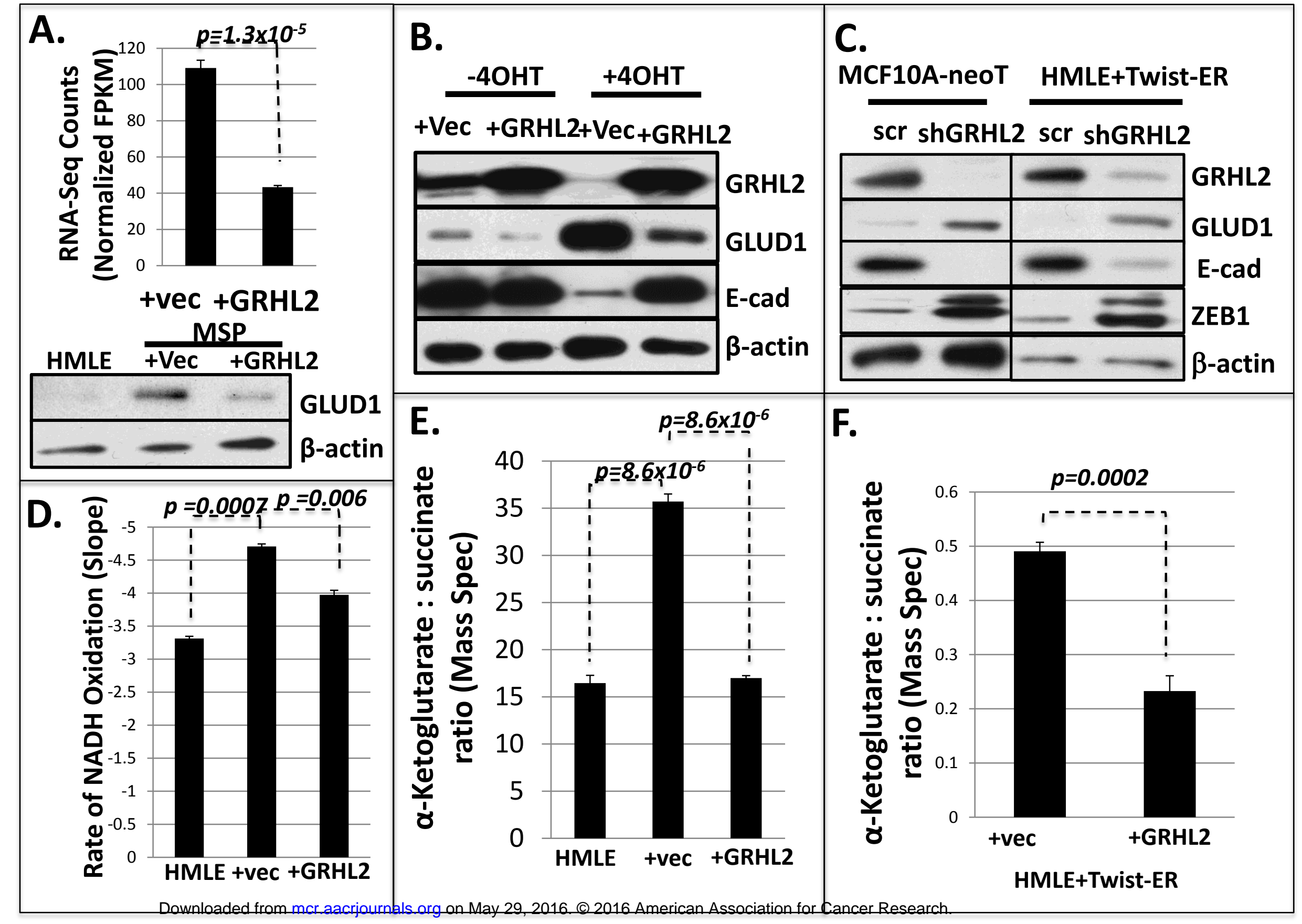




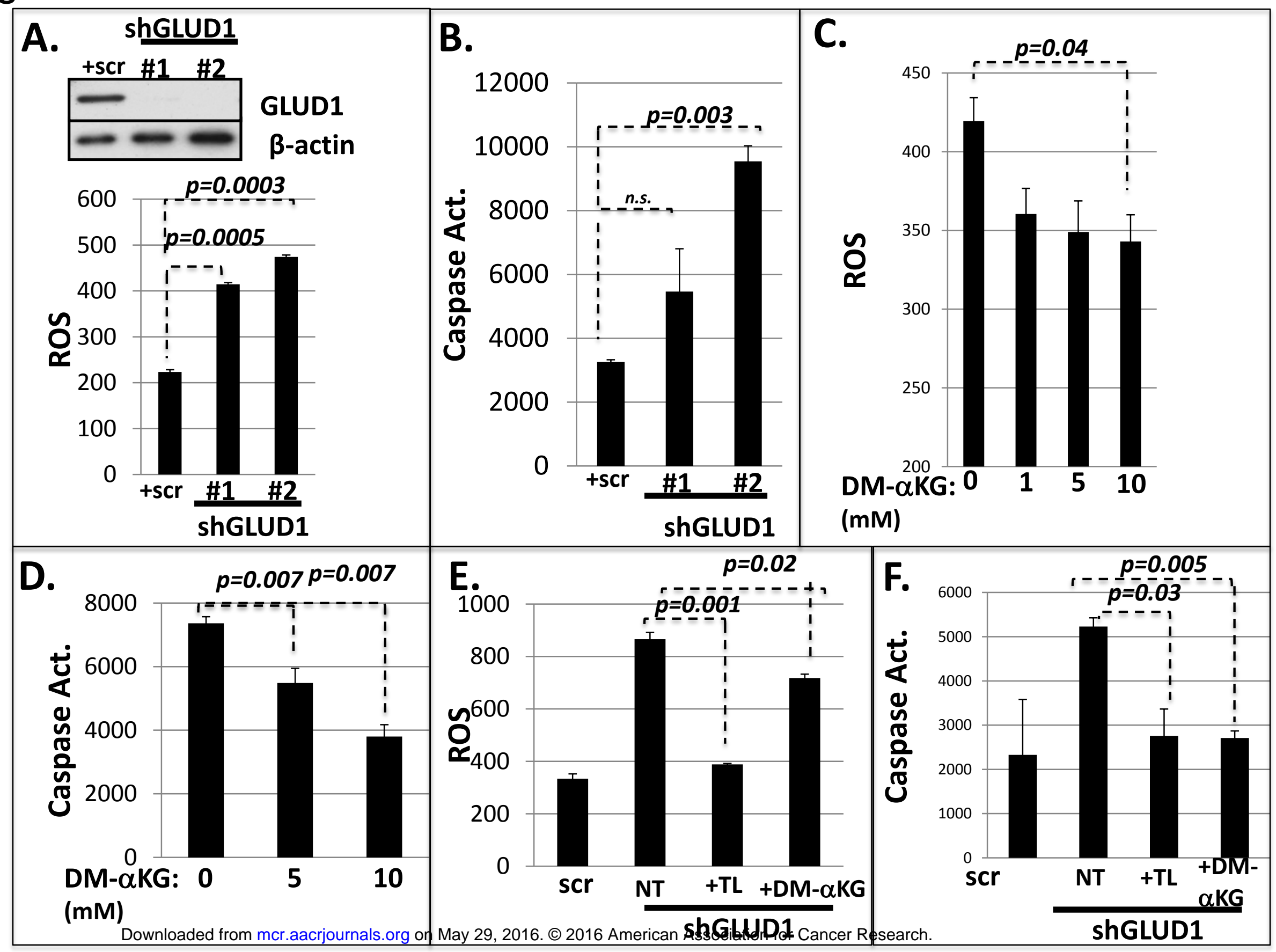

Figure 6 


\section{Figure 7}

\section{GRHL2}

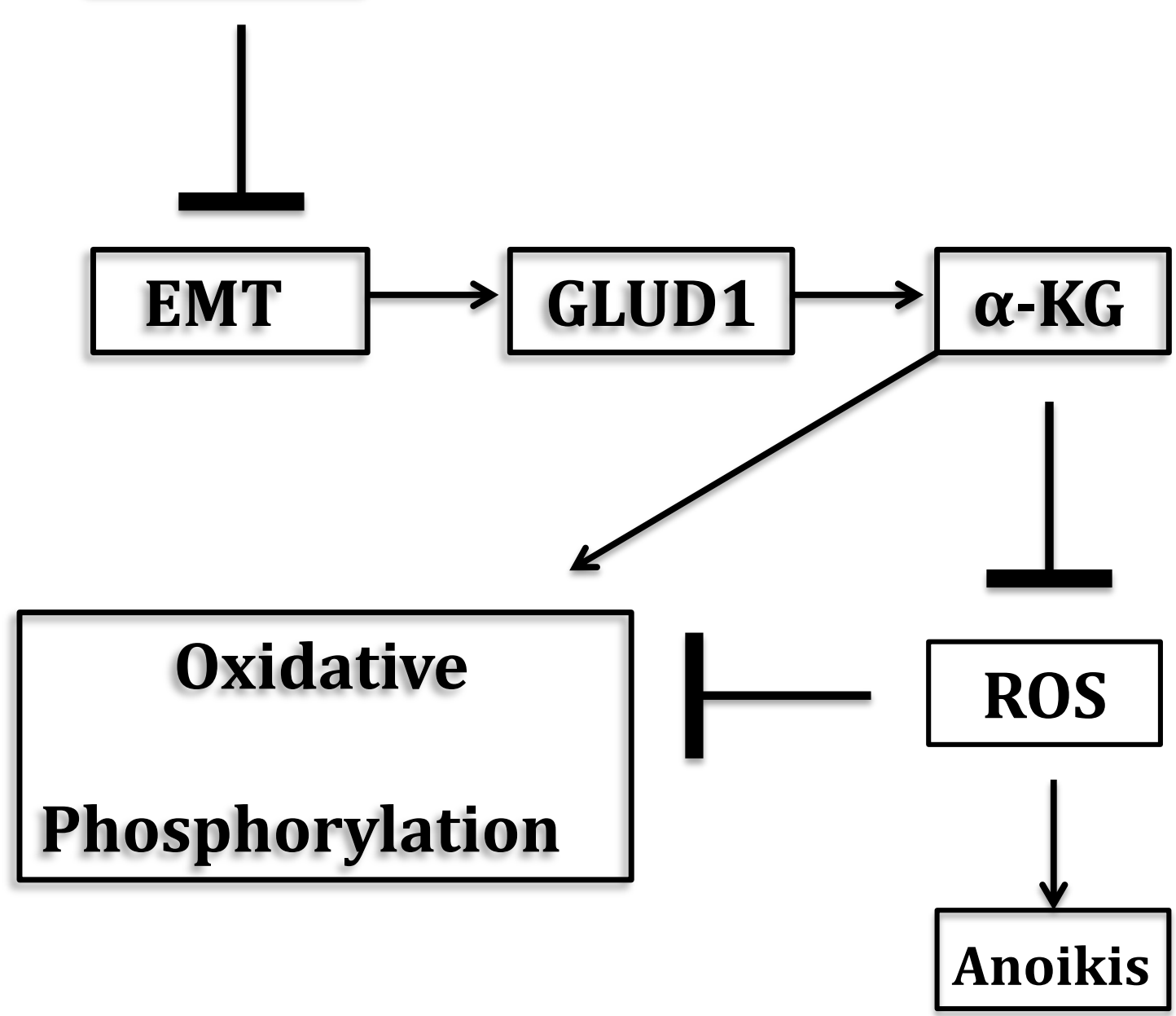




\section{Molecular Cancer Research}

\section{Grainyhead-like 2 Reverses the Metabolic Changes Induced by the Oncogenic Epithelial-mesenchymal Transition: Effects on Anoikis}

Joshua C. Farris, Phillip M. Pifer, Liang Zheng, et al.

Mol Cancer Res Published OnlineFirst April 15, 2016.

$\begin{array}{ll}\text { Updated version } & \begin{array}{l}\text { Access the most recent version of this article at: } \\ \text { doi:10.1158/1541-7786.MCR-16-0050 }\end{array}\end{array}$

Supplementary Access the most recent supplemental material at:

Material http://mcr.aacrjournals.org/content/suppl/2016/05/13/1541-7786.MCR-16-0050.DC1.html

Author Author manuscripts have been peer reviewed and accepted for publication but have not yet been Manuscript edited.

E-mail alerts Sign up to receive free email-alerts related to this article or journal.

Reprints and To order reprints of this article or to subscribe to the journal, contact the AACR Publications Subscriptions Department at pubs@aacr.org.

Permissions To request permission to re-use all or part of this article, contact the AACR Publications Department at permissions@aacr.org. 